\title{
Comprehensive Morphological and Transcriptomic Analysis of the Chemosensory System in the Red Flour Beetle, Tribolium castaneum
}

\author{
Dissertation \\ for the award of the degree \\ "doctor rerum naturalium" \\ of the Georg-August-Universität Göttingen \\ within the doctoral program "Biology" \\ of the Georg-August-University School of Science (GAUSS)
}

submitted by

Stefan Dippel

from Marburg,

Germany 
Thesis committee

\section{Prof. Dr. Ernst A. Wimmer}

Department of Developmental Biology

Georg-August-University, Göttingen

\section{Prof. Dr. André Fiala}

Department of Molecular Neurobiology of Behavior

Georg-August-University, Göttingen

\section{Members of the Examination Board}

\section{$1^{\text {st }}$ Reviewer}

Prof. Dr. Ernst A. Wimmer

Department of Developmental Biology

Georg-August-University, Göttingen

\section{$2^{\text {nd }}$ Reviewer}

Prof. Dr. André Fiala

Department of Molecular Neurobiology of Behavior

Georg-August-University, Göttingen

Further members of the Examination Board

Dr. Roland Dosch

Department of Developmental Biochemistry

University Medical Center Göttingen

Dr. Ivan Manzini

Department of Neurophysiology and Cellular Biophysics

Georg-August-University Göttingen

Prof. Dr. Ralf Heinrich

Department of Cellular Neurobiology

Georg-August-University Göttingen

Prof. Dr. Joachim Schachtner

Department for Biology - Neurobiology/Ethology

Philipps-University Marburg 
Affidavit

Herewith I declare, that I prepared the Dissertation

"Comprehensive Morphological and Transcriptomic Analysis of the Chemosensory System in the Red Flour Beetle, Tribolium castaneum"

on my own and with no other sources and aids than quoted.

Göttingen, August 2016

Stefan Dippel 
"Everything will be okay in the end. If it's not okay, it's not the end."

John Lennon 


\section{Content}

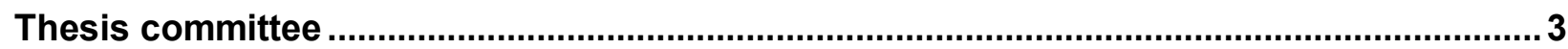

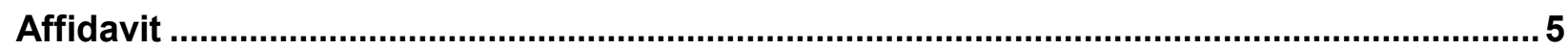

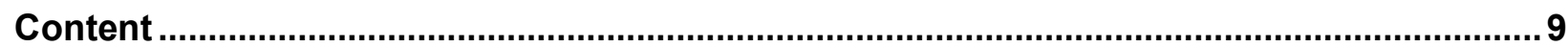

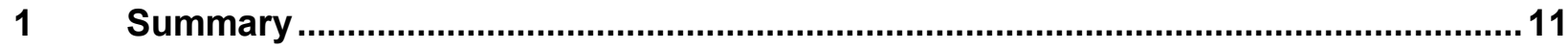

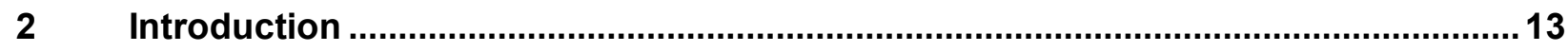

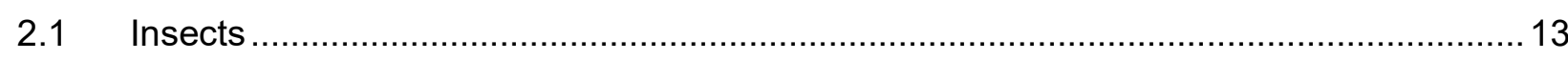

2.2 Tribolium castaneum as a model system for beetle olfaction ....................................... 13

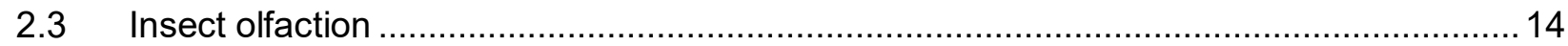

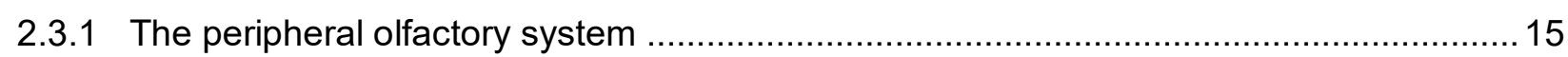

2.3.2 The olfactory and gustatory sensory neurons ...................................................... 16

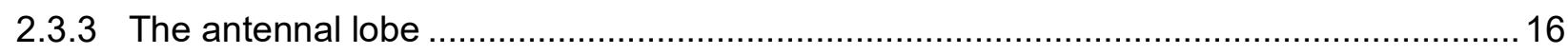

2.3.4 Higher integration centers for chemosensory input - mushroom bodies and lateral

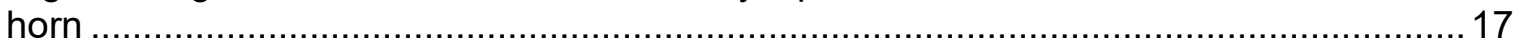

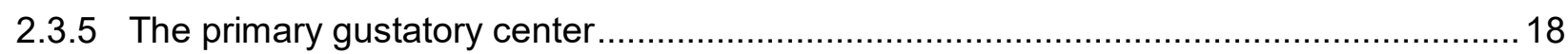

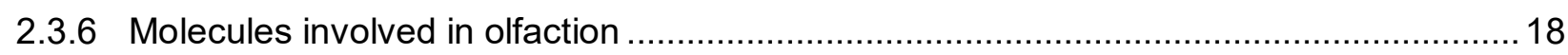

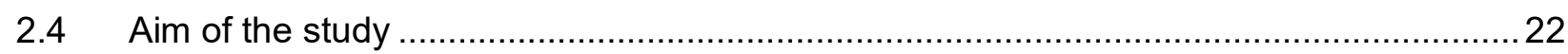

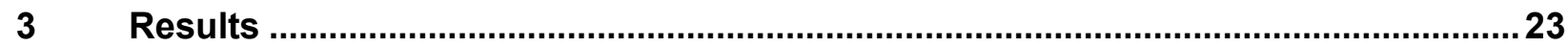

3.1 Tissue-specific transcriptomics, chromosomal localization, and phylogeny of chemosensory and odorant binding proteins from the red flour beetle Tribolium castaneum reveal subgroup specificities for olfaction or more general functions

3.2 Morphological and Transcriptomic Analysis of a Beetle Chemosensory System Reveals a Gnathal Olfactory Center.

3.3 Improvement of trangenesis in $T$. castaneum and establishment of an additional binary expression system (work in progress) ........................................................... 92

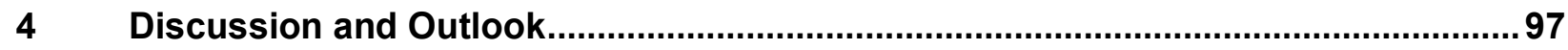

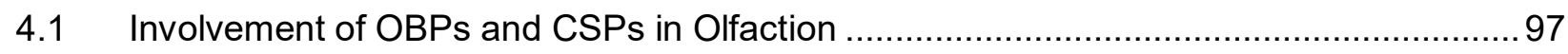

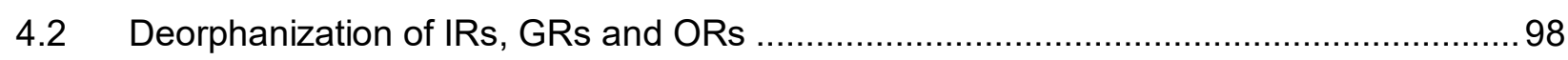

4.3 The innervation of the $A L$ and possible exceptions from the 'Central dogma' .............100

4.4 Environmental or age dependent regulation of OR expression ................................... 101

4.5 The gnathal olfactory center and its function ........................................................ 102

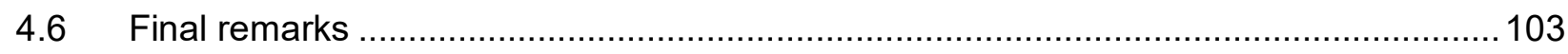

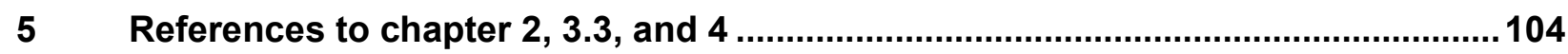

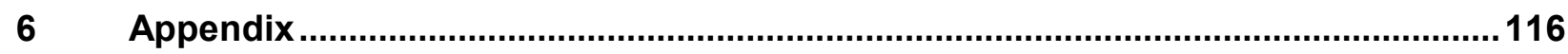

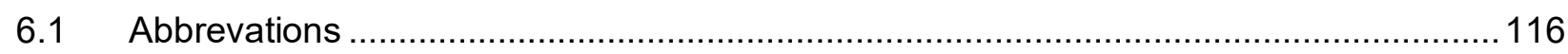

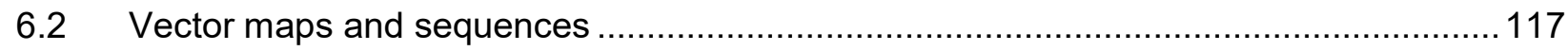

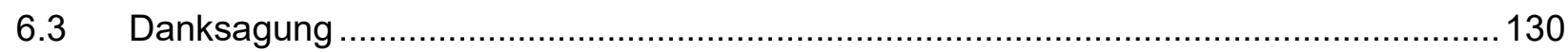

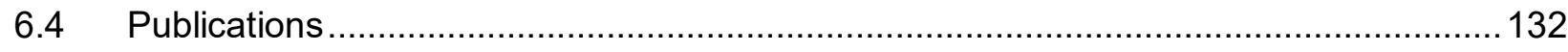




\section{Summary}

The red flour beetle, Tribolium castaneum is an emerging coleopteran model system and thus represents the largest insect order with several agricultural and forest pests. Despite the ecological and economic importance of beetles, little has so far been known about the molecular and anatomical basis of their olfactory system.

My genome-wide expression analysis in the antennae, mouthparts, legs, heads and bodies have gained insights into the number and distribution of genes relevant for chemosensation. The combination with an interspecies phylogenetic comparison with Drosophila melanogaster and Anopheles gambiae has confirmed or revealed additional details about the conservation of these genes in $T$. castaneum.

In the olfactory sensilla, odors first interact with odorant binding proteins, which are believed to translocate mostly hydrophobic odors through the aqueous sensilla lymph to the receptors. We found subgroups of these odorant binding proteins (most of the classical odorant binding proteins and all antennal binding proteins II) to be highly expressed in antennae and therefore likely involved in chemoperception. Mainly three receptor families are responsible for the perception of odor in the chemosensory neurons. I confirmed that the 'antennal' ionotropic glutamate-like receptors, which were shown to detect, for example organic acids, aldehydes, and amines, but also temperature and humidity in D. melanogaster, are highly conserved across species and abundant in $T$. casatneum antennae. Therefore, they are most likely involved in $T$. castaneum olfaction, in contrast to the 'divergent' ionotropic glutamate-like receptors, which are not enriched in antennae. In addition, I could confirm that the gustatory and odorant receptors of $T$. castaneum underwent a substantial radiation on the way to $T$. castaneum, leading to the exceptionally high receptor numbers, compared to other species. Only few of the $T$. castaneum gustatory receptors $\left(\mathrm{CO}_{2}\right.$, sugar, and fructose receptors), the odorant receptor coreceptor (Orco), but no typical odorant receptors, have clear orthologs in the analyzed dipterans. I found the total number of expressed odorant receptors to be lower than the amount of genes encoding intact odorant receptors, which implicates an age dependent or environmental regulation of odorant receptor expression. Furthermore, the distribution of the gustatory and odorant receptors expression revealed high numbers of both receptor types to be expressed in both antennae as well as palps. This indicates that there is no organotopic separation of olfaction and gustation between antennae and palps, which is in contrast to the textbook knowledge of insect chemoperception.

Antennal and palpal backfills, as well as a transgenic line that labels olfactory sensory neurons revealed that the olfactory sensory input from the antennae and palps is mostly processed separately in three independent neuropils. The antennal olfactory information is processed in the ipsilateral antennal lobe, the first integration center of olfactory input in the brain, as described in other insect orders. It was speculated that olfactory sensory neurons that express 
the same odorant receptor project into the same distinct antennal lobe substructure (glomerulus). However, in T. castaneum the number of antennal expressed odorant receptors exceeds the amount of antennal lobe glomeruli, indicating that coexpression or convergence of more than one olfactory sensory neuron per glomerulus is not just an exception. From the antennal lobe distinct tracts formed by projection neuron relay the olfactory information into higher integration centers in the brain. We revealed, through the use of dye injections into the antennal lobe, that $T$. castaneum also has three antennal lobe tracts that connect to the mushroom bodies and the lateral horn. This has been described for other holometabolous insects, but was previously only speculated for beetles. In contrast to the antennal olfactory input, the palpal olfactory projections innervate the ipsilateral lobus glomerulatus, which is located close to, but outside the antennal lobe and has previously only been described in hemimetabolous insects. Besides this lobus glomerulatus, we identified a second, so far undescribed neuropil responsible for processing palpal derived olfactory input: an unpaired and glomerular organized neuropil in the gnathal ganglion, which we termed "gnathal olfactory center".

In addition to this novel finding, the presented detailed genome-wide expression analysis of chemosensory related genes and morphological description of the olfactory pathway in T. castaneum present the important and essential groundwork for future studies to better understand coleopteran olfaction. 


\section{Introduction}

\section{$2.1 \quad$ Insects}

Insects are with about 1.4 million described species the largest class of the animal kingdom (Chapman, 2009) and are adapted to more or less all terrestrial niches (Grimaldi and Engel, 2005). Due to their small size and often hidden lifestyle their ecological and economic impact is usually underestimated. However, they are in fact essential for a functional ecosystem, playing viatl roles in, for example, the degradation of detritus and reconditioning soils, pollination of flowering plants, and being the basis of most terrestrial food chains (Shurin et al., 2006; Zhang et al., 2007). Besides these benefits, many insects are harmful

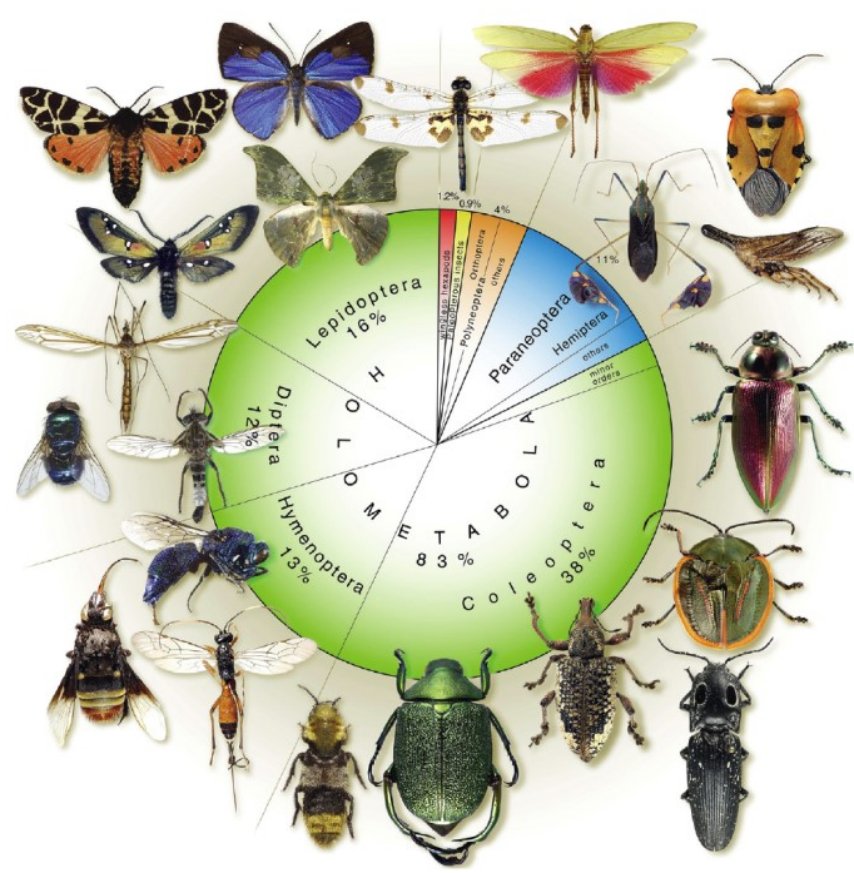

Figure 2.1. Number of insect species per order, from Grimaldi and Engel, 2005 pests, as they destroy wooden infrastructure, forests, crops and stored agricultural products (Pedigo and Rice, 2014), as well as transmit several diseases such as malaria, dengue and the Zika virus (Benelli and Mehlhorn, 2016). Within the insects, the beetles are, with more than 400,000 species (Figure 2.1), the largest order and contain consequently many pests, such as the Colorado potato beetle (Leptinotarsa decemlineata), pollen beetle (Brassicogethes aeneus), bark beetles (Dendroctonus ponderosae, Ips typographus), red palm weevil (Rhynchophorus ferrugineus), western corn rootworm (Diabrotica virgifera), long-horned beetles (Hylotrupes bajulus, Anoplophora glabripennis), and the red flour beetle (Tribolium castaneum). Despite the ecological and economic importance of beetles, little was known about the molecular and anatomical basis of their olfactory system. Therefore, we started to investigate $T$. castaneum as a representative of this important order to increase our knowledge about their chemosensory system.

\subsection{Tribolium castaneum as a model system for beetle olfaction}

$T$. castaneum and $T$. confusum are probably the most common secondary pests for all kinds of globally stored agricultural products (Brown et al., 2009; Sallam and Mejida, 2008). Hints from archeological records of Egyptian tombs (Buckland, 1981) point to an African origins. Field observations have revealed that they naturally live under the bark of trees, in dead wood or in 


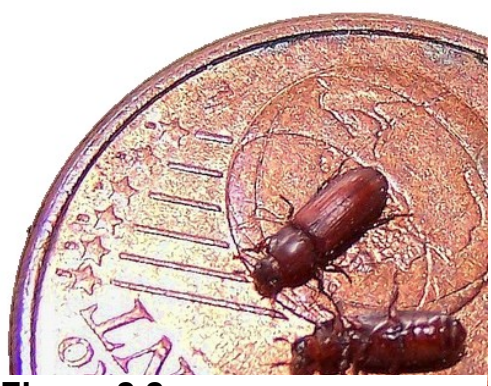

Figure 2.2.

Tribolium castaneum, on top of a one cent coin.

hymenopteran hives (Arnaud et al., 2005). Under laboratory conditions, Tribolium is easy to maintain, is small with minimal space requirements (Figure 2.2), has a short generation time, and a high reproduction rate (Berghammer et al., 1999a; Sokoloff, 1972), which turned it into a popular model system besides Drosophila. Additionally, the published fully annotated genomic sequence (Kim et al., 2010; Richards et al., 2008; Wang et al., 2007), powerful reverse genetics by systemic RNA Interference (Bucher et al., 2002; Schmitt-Engel et al., 2015; Tomoyasu and Denell, 2004), the established transgenesis systems (Berghammer et al., 1999b), by random transposon mutagenesis (Lorenzen et al., 2003; Trauner et al., 2009), or Crispr/Cas9 mediated knock-in (Gilles et al., 2015), as well as the heat-shock induced or the Gal4/UAS missexpression systems (Schinko et al., 2010, 2012) transform T. castaneum into a prime model system for coleopteran pest species. This enables a re-evaluation of results from $D$. melanogaster for their generality in insects (Dippel et al., 2014). However, some of the published tools are still in initial stages with no "out of the box" solutions and have potential for further improvements, which is an additional aspect of this thesis.

Many Tenebrionidae, including T. castaneum, produce methyl- and ethyl-1,4-benzoquinone as major volatile, defense secretion, and antimicrobial substances, which are stored and contained in prothoracic and pygidial glands (Howard, 1987; Suzuki et al., 1988, 1988; Verheggen et al., 2007; Yezerski et al., 2007). Moreover, the quinones, as well as 4,8-dimethyldecanal (DMD) (Faustini et al., 1981), which is produced by adult males in the setiferous glands on the femora (Faustini et al., 1981, 1982) and possibly also in other tissues (Arnaud et al., 2002; Bloch Qazi et al., 1998), serve as aggregation pheromones. Despite the production of pheromonal substances, no sex specific attraction to the aggregation pheromone 4,8-Dimethyldecanal (Suzuki, 1980) and no sex preference in the mating choice of males (Serrano et al., 1991) has been observed. However, Tribolium's visual system is with about 68 Ommatidia per eye (Friedrich et al., 1996) and a relatively small optical lobe (Dreyer, 2010), less complex compared to other insects and therefore possibly of minor relevance. This indicates a more prominent role for chemo perception in this species, which is an additional reason to investigate $T$. castaneum olfaction.

\subsection{Insect olfaction}

The survival of insects strongly depends on their ability to receive and interpret minute amounts of chemicals from their environment to find food sources, kin and mates, hosts and oviposition sites, avoid predators, toxins, and harmful microbesas well as to communicate intra- and interspecifically (Dicke, 2009; Laska et al., 1999; Linz et al., 2013; Liu et al., 2008; 
Paczkowski et al., 2014; Stensmyr et al., 2012; Sun et al., 2012; Weiss et al., 2011; Whiteman and Pierce, 2008; Yang et al., 2008). The olfactory and gustatory system is precisely tuned at various levels to translate the composition, concentration and spatio-temporal distribution of chemical stimuli into innate or learned behavior. This process begins with the perception of semiochemicals at the periphery, followed by the processing of this sensory input in the antennal lobes (ALs) or the primary gustatory center (PGC), and eventually integration in the higher processing centers of the brain, such as the mushroom bodies (MBs) and the lateral horns (LHs) (Belle and Heisenberg, 1994; Galizia and Sachse, 2010; Leal, 2013; Sachse, 2014).

Most of our knowledge is derived from just a few model species e.g. bees, moths, mosquitos, locusts, and mostly from the vinegar fly (Farris and Van Dyke, 2015; Szyszka and Galizia, 2015), but not from beetles. Consequently, many statements about 'insect olfaction' are based on generalizations and assumptions instead of science-based knowledge.

\subsubsection{The peripheral olfactory system}

The perception of chemical stimuli starts at the olfactory sensilla (Figure 2.3) located on the third antennal segment and the maxillary palps of D. melanogaster (Martin et al., 2013; Shanbhag et al., 1999). Based on their morphology the olfactory sensilla can be subdivided into three major subtypes: the basiconic, trichoid, and coeloconic sensilla (Galizia and Sachse, 2010). These are hair like structures with a porous cuticular wall that allows the odors to pass through and

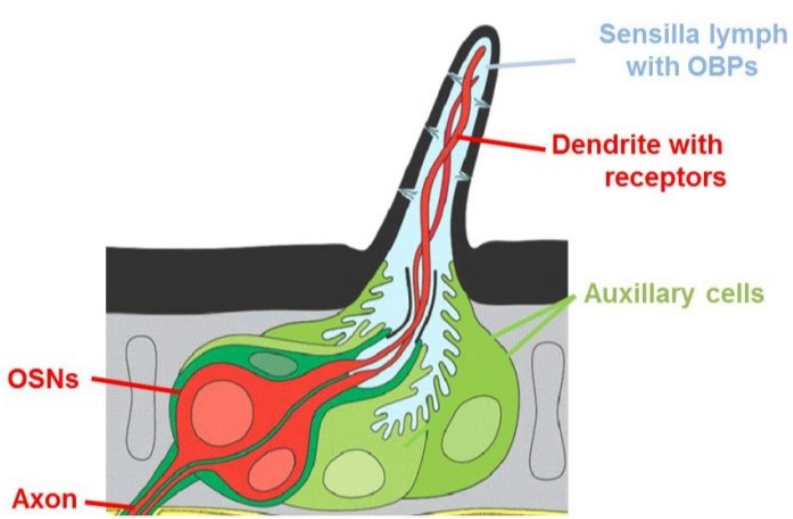

Figure 2.3. Diagram of a moth olfactory sensillum trichodeum with two receptor cells (red) and three auxiliary cells (green). In blue: Sensillum lymph, after Kaissling, 2004. enter an aqueous lymph filled central cavity (Steinbrecht, 1996). Within this cavity the reception of odors takes place via the chemoreceptors embedded in their dendritic membrane of the olfactory sensory neurons (OSNs). The sensilla lymph, which surrounds the dendritic branches of the OSNs, contains high amounts of odorant binding proteins (OBPs), chemosensory proteins (CSPs), and odorant degrading enzymes (ODEs). These proteins are produced and secreted by adjacent auxiliary cells (Leal, 2013; Pelosi et al., 2014). Besides the production and homeostasis of the sensilla lymph, the auxiliary cells are also involved in the secretion of the cuticular sensillum during development and later on insulate the lymphatic space including the central cavity of the sensillum (Hansson, 2013). 


\subsubsection{The olfactory and gustatory sensory neurons}

The OSNs and GSNs are primary neurons, with dendritic branches in the adjacent sensilla cavity and axons converging to the antennal or palpal nerves that typically innervate the AL or the PGC in the gnathal ganglion, respectively (Leal, 2013; Miyazaki and Ito, 2010; Montell, 2009; Schachtner et al., 2005). The number of OSNs/GSNs per sensillum can vary between the sensilla types of a species and between different species (Doty, 2015). The type, as well as response profile of each chemosensory neuron is defined by the type of chemoreceptor expressed. In insects mainly three receptor families are involved in the translation of a chemical stimulus into neuronal activity: the ionotropic glutamate-like receptors (IRs) (Benton et al., 2009), the gustatory receptors (GRs) (Montell, 2009; Weiss et al., 2011), and the odorant receptors (ORs) (Missbach et al., 2014; Sato et al., 2008; Wicher et al., 2008).

\subsubsection{The antennal lobe}

The sensory information received by the antennal OSNs is forwarded via the antennal nerve into the $A L$ (Figure 2.4), the first integration center of odors in the cerebral ganglion (Hansson, 2013; Schachtner et al., 2005). The AL of insects is typically compartmentalized in spherical regions of high synaptic density, the so called olfactory glomeruli (Schachtner et al., 2005). Based on findings in $D$. melanogaster it is assumed that most OSNs express only one type of OR besides the atypical odorant receptor coreceptor (Orco) and all OSNs expressing the same typical OR converge into the same olfactory glomerulus (Jefferis et al., 2001;

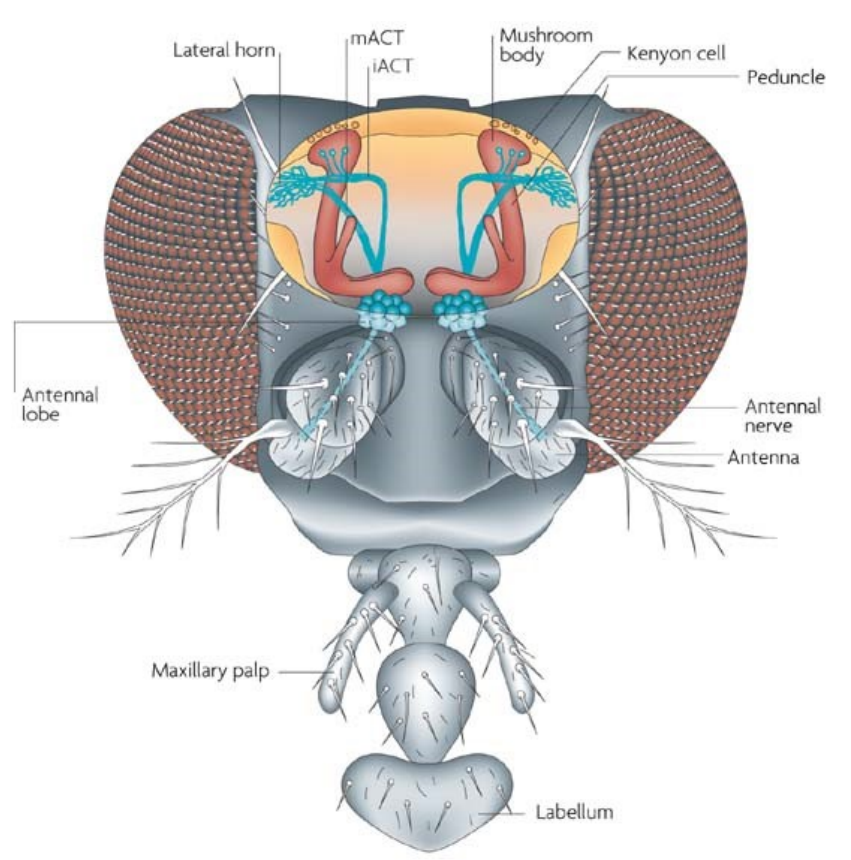

Figure 2.4. Dorsal view of a fly head showing the main elements of the olfactory pathway, from Keene \& Waddell, 2007 Keller and Vosshall, 2003; Stocker, 2001; Vosshall, 2000), known as the central dogma of olfaction (Jefferis, 2005; Smith, 2008). Within the AL the incoming sensory input is processed by a complex network of local interneurons (LNs), centrifugal neurons, and feedback from the projection neurons (PNs) (Hu et al., 2010; Schachtner et al., 2005). The LNs mainly release GABA, but also acetylcholine (Chou et al., 2010), and several neuropeptides (Binzer et al., 2014; Schachtner et al., 2005) to shape and refine the olfactory representation within and between glomeruli. 


\section{Holometabola}
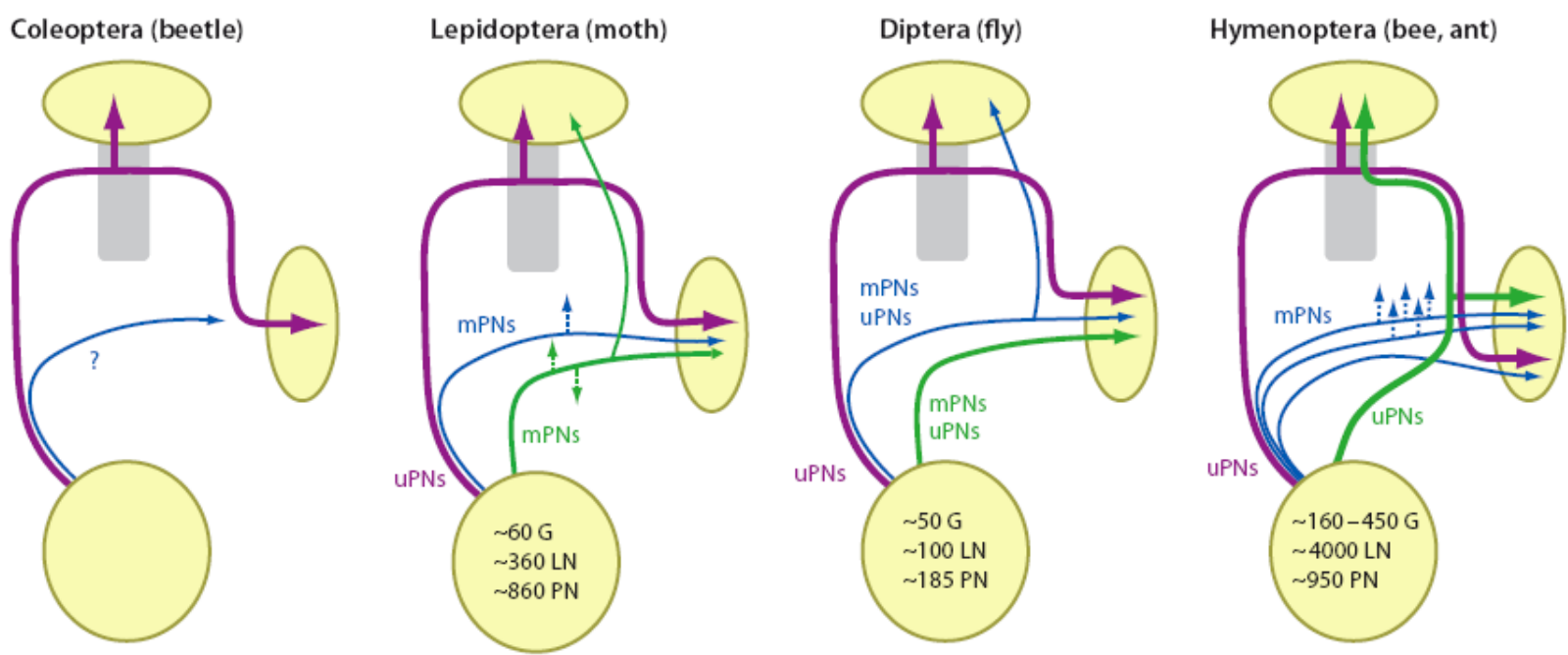

Figure 2.5. Organaization of the antennal lobe tracts (ALTs) in different holometabolous orders, the lack of data from coleopteran is nicely reflected in this schematic. mPNs, multiglomerular PNs; uPNs, uniglomerular PNs; G, glomeruli; LN, local interneurons; PN, projection neurons; from Galizia and Rössler, 2010.

In holometabolous insects the PNs usually form three distinct axon bundles (Figure 2.5), the AL tracts (ALT), which relay the olfactory information to higher brain centers like the mushroom bodies and the lateral horn (Galizia and Rössler, 2010). The PNs that branch in a single glomerulus (uniglomerular) usually send excitatory (cholinergic) projections to the MB and the $\mathrm{LH}$, whereas the multiglomerular PNs tend to be inhibitory (GABAergic) and target only the LH (Galizia, 2014).

\subsubsection{Higher integration centers for chemosensory input - mushroom bodies and lateral horn}

The insect MBs are paired protocerebral neuropils formed by the intrinsic Kenyon cells (KCs) and projections from several sensory pathways (Campbell and Turner, 2010). The MB consists of the dendritic input region, the calyx, as well as the axon bundles of the KCs forming the pedunculus and its lobes. Within the MB lobes, afferent input to and efferent output from the KCs are relayed from or to extrinsic neurons (Ito et al., 1998; Tanaka et al., 2008). The MBs are higher integrative centers that are considered to be largely responsible for olfactory discrimination (Heuer et al., 2012), olfactory associative learning, and memory formation (Farris and Van Dyke, 2015; Fiala, 2007). Besides processing of olfactory information, evidence from several insect species indicates that other sensory modalities (e.g. visual, gustatory, mechanosensory, and acoustic) are also integrated (Heuer et al., 2012; Vogt et al., 2016). Additionally, the MBs play a crucial role in locomotor activity (Martin et al., 1998) and sleep (Bushey and Cirelli, 2011) as demonstrated in D. melanogaster. 
The lateral horn is a paired neuropil in the lateral protocerebrum which decodes odor quality and intensity through the integration of $\mathrm{AL}$ output from inhibitory and excitatory projection neurons towards innate decisions (Strutz et al., 2014). In some species the LH is morphologically distinguishable from the surrounding tissue, but not in all, therefore some authors prefer the term 'lateral protocerebrum'. The LH of D. melanogaster gets direct olfactory input from the $A L$ via GABAergic PNs forming the mIALT and cholinergic PNs of the IALT and indirectly via the mALT, which passes the MB (Strutz et al., 2014).

\subsubsection{The primary gustatory center}

Gustatory information in D. melanogaster is mainly processed in the PGC, a neuropil in the gnathal ganglion, which can be subdivided into zones (Miyazaki and Ito, 2010). In $D$. melanogaster the gustatory information from the PGC is somehow relayed to the mushroom bodies were the integration, as well as the gustatory learning takes place (Kirkhart and Scott, 2015). So far the only evidence for gustatory projection neurons from the GNG to the MBs comes from data in the honeybee, in which these projections innervate similar regions in the ipsilateral calyx, as shown for the olfactory projections (Schröter and Menzel, 2003).

\subsubsection{Molecules involved in olfaction}

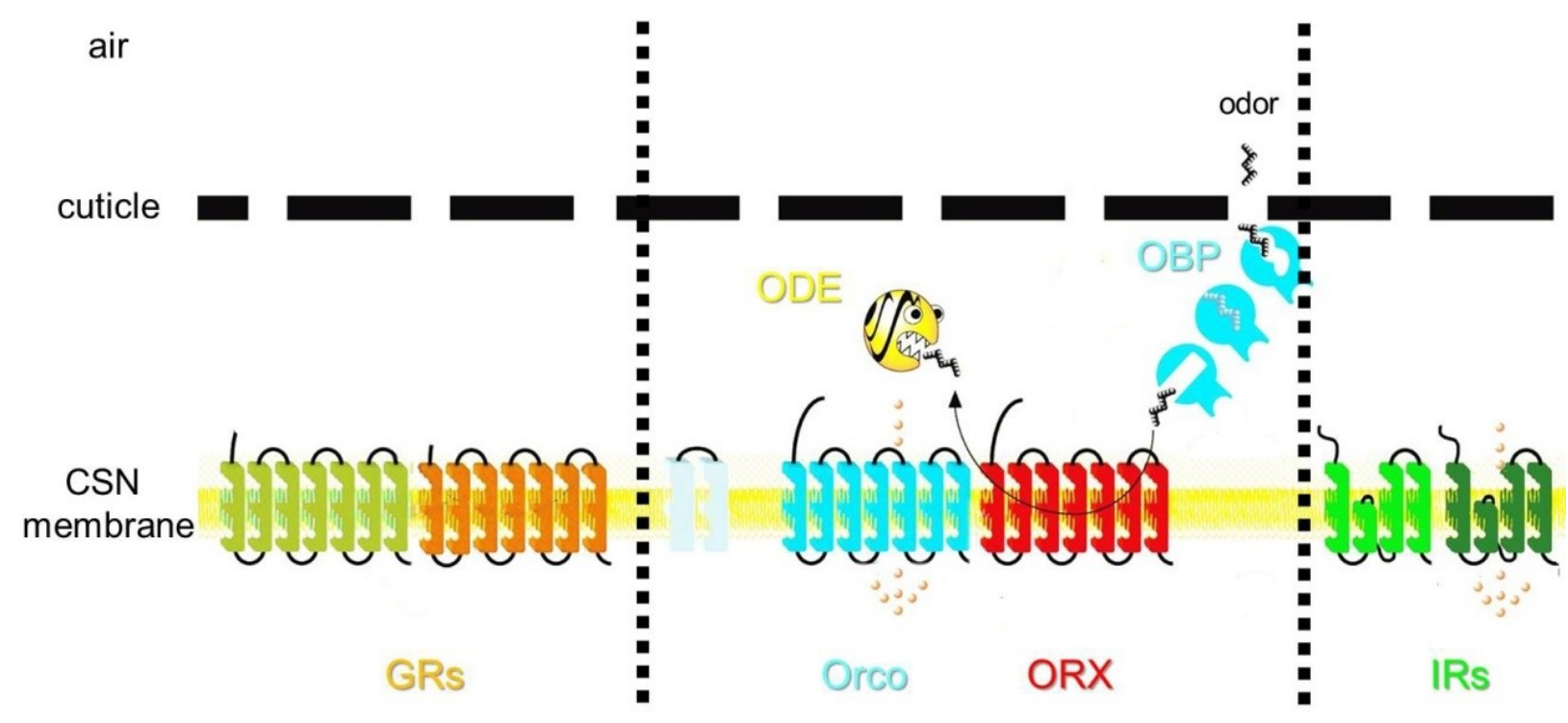

Figure 2.7. Hypothetical and simplified model of the main protein families/classes involved in chemoperception. The different receptor families usually do not occur in the same neuron. CSN, chemosensory neuron; GRs, gustatory receptors; ODE, odorant degrading enzyme, Orco, odorant receptor coreceptor; ORX, typical odorant receptor; OBP, odorant binding protein; IRs, ionotropic glutamate-like receptors. Massively modified, after Rützler and Zwiebel, 2005. 


\subsubsection{Odorant binding proteins and chemosensory proteins}

The detection of airborne odorants requires translocation from the sensilla pores, through the aqueous sensilla lymph, to the dendritic chemoreceptors, which is allegedly performed by OBPs and CSPs (Figure 2.7). OBPs are only found in insects, whereas CSPs are already present in other arthropods (Pelosi et al., 2014; Vieira and Rozas, 2011). It is therefore likely that OBPs are derived from proto-CSPs after the Hexapoda-Crustacea split (Vieira and Rozas, 2011). The OBPs can be subdivided based on their phylogeny into classic OBPs, antennal binding proteins (ABPs), C+OBPs, C-OBPs, and dimeric OBPs (Vieira and Rozas, 2011). Some authors also use the term 'pheromone binding protein' to highlight the function of some OBPs (Vogt and Riddiford, 1981), although they are not conserved across insect orders.

OBPs and CSPs are small (10 to $30 \mathrm{kDa}$ ), globular, and water soluble proteins (Vieira and Rozas, 2011) and highly abundant in the aqueous sensilla lymph (Steinbrecht, 1998; Vogt and Riddiford, 1981). The CSPs are defined by four, the OBPs by six highly conserved cysteine residues (Briand et al., 2002; Vieira and Rozas, 2011), which form two or three interlocking disulfide bridges, leading to the exceptional stability of these proteins (Briand et al., 2002; Sandler et al., 2000; Scaloni et al., 1999). OBPs, as well as CSPs form a hydrophobic inner pocket to bind potential odorants (Sandler et al., 2000). It is widely accepted that OBPs bind and solubilize hydrophobic odors, translocate them through the aqueous lymph, and thereby contribute to the sensitivity of the olfactory system (Leal, 2013). Several studies implicate that OBPs form functional dimers (Andronopoulou et al., 2006) with cooperative binding properties with substantial differences between the homo- and heterodimers (Qiao et al., 2011; Wang et al., 2013). However, expression studies in several insect species showed that many CSPs and OBPs are not restricted to chemosensory tissues (Farhadian et al., 2012; Gong et al., 2007; Pelletier and Leal, 2009; Pitts et al., 2011; Zhang et al., 2013a; Zheng et al., 2013; Zhou et al., 2006) and are also involved in the release of semiochemicals (Dani et al., 2011), mating (Sun et al., 2012), embryogenesis (Maleszka et al., 2007), immune-response (Levy et al., 2004), and regeneration (Nomura et al., 1992).

\subsubsection{Odorant degrading enzymes}

Proper detection of chemicals with a high temporal resolution requires besides a fast detection also a fast signal termination, which is presumably facilitated by ODEs (Durand et al., 2011; Ishida and Leal, 2005; Younus et al., 2014). Every enzyme within the sensilla lymph, able to cleave or modify odorants, can potentially serve as an ODE. Consequently, ODEs belonging to various protein families e.g. aldehyde dehydrogenase (ALDH), aldehyde oxidase (ALOX), carboxylesterase (CES), epoxide hydrolase (EH), glutathione S-transferase (GST), and cytochrome P450 (CYP) were identified in several species (Leal, 2013). 


\subsubsection{The ionotropic glutamate-like receptors (IRs)}

The IRs are evolutionarily the oldest of the three main chemoreceptor families in insects, already present in several protostomes (Benton et al., 2009). Within the insects, the 'antennal IRs' and especially the coreceptors IR8a and IR25a are highly conserved and are typically expressed in OSNs associated with coeloconic sensilla (Croset et al., 2010; Rytz et al., 2013). The functional receptors are heteromeric complexes between odor specific IRs and a coreceptor, shown to be essential for proper functionality as well as the dendritic localization of these complexes (Croset et al., 2010; Rytz et al., 2013). The IRs typically consist of three transmembrane domains, of which two form the ion channel, a bipartite ligand-binding domain, and in the case of the coreceptors an amino-terminal domain which possibly enables hetero-complex formation (Croset et al., 2010; Rytz et al., 2013). The OSNs of $D$. melanogaster that express IRs, typically respond to water soluble odors like acids, aldehydes, and amines (Rytz et al., 2013), but also to salt (Zhang et al., 2013b), temperature, and humidity (Enjin et al., 2016). In D. melanogaster, almost all OSNs expressing the same specific IR converge in a single $\mathrm{AL}$ glomerulus and form a map like representation of sensory input. Despite the scattered distribution of the different sensilla on the antenna, all IR innervate glomeruli are clustered within the AL (Rytz et al., 2013), from which PNs ascent into higher integration centers, the mushroom body and lateral horn (Silbering et al., 2011) .

\subsubsection{The gustatory receptors (GRs)}

The GRs can be found in several arthropods (Cao, 2014; Chipman et al., 2014; Chyb, 2004; Sánchez-Gracia et al., 2001), they are seven transmembrane receptors (Freeman et al., 2014; Jiao et al., 2008; Lee et al., 2009; Weiss et al., 2011), and in D. melanogaster they are responsible for the detection of sugars, $\mathrm{CO}_{2}$, several bitter components (e.g. caffeine), pheromones (Isono and Morita, 2010), and warmth avoidance (Ni et al., 2013). GRs are often coexpressed with other GRs in single receptor neurons (Isono and Morita, 2010), but contradicting reports about their quaternary structures ranging from homodimers to heteromultimers (Freeman et al., 2014; Jiao et al., 2008; Lee et al., 2009; Weiss et al., 2011) indicate complex cooperation. Insect GRs are unrelated to mammalian G-protein coupled taste receptors, but distantly related to insect ORs, which are likely to be odorant-gated cation channels (Shim et al., 2015). However, the transduction mechanism of GRs is still under debate (Ishimoto et al., 2005; Sato et al., 2011). In D. melanogaster the majority of the GSNs are located in the labellum, but also on most other body parts like the antennae, legs, and wings (Depetris-Chauvin et al., 2015). The labellar GSNs project into the primary gustatory center in the gnathal ganglion (Miyazaki and Ito, 2010), whereas the antennal $\mathrm{CO}_{2}$ sensitive Neurons innervate a single AL glomerulus (Kwon et al., 2007). 


\subsubsection{The odorant receptors (ORs)}

The functional insect odorant receptors are heteromeric complexes formed by two seven transmembrane proteins, a typical odorant specific OR and the atypical odorant receptor coreceptor (Orco) (Krieger, 2003; Mukunda et al., 2014; Vosshall and Hansson, 2011) which is highly conserved across insects (Missbach et al., 2014). The ORs are distantly related to the arthopod GRs (Clyne et al., 1999; Scott et al., 2001) and unrelated to vertebrate ORs, reflected, for example by their inverted membrane topology (Benton et al., 2006; Lundin et al., 2007). The ORs seem to be evolved stepwise, first Orco in the Dicondylia lineage leading to at least three Orco-paralogues in Thermobia domestica, followed by the typical ORs in the pterygote insects which possess only one Orco but plenty of typical ORs (Missbach et al., 2014). The Orco/OR complexes form odor gated cation channels, which are tuned by intracellular signaling (Mukunda et al., 2014). Orco is essential for the correct integration of typical ORs into the dendritic membranes of OSNs (Larsson et al., 2004) and it contributes to the OR ion channel pore formation (Mukunda et al., 2014). The typical ORs are highly diverse, usually tuned to a wide variety of chemicals, but also highly specialized - mostly pheromone sensitive - ORs are described (Andersson et al., 2015; Galizia et al., 2010). 


\subsection{Aim of the study}

The aim of this study was to establish the first detailed description of the olfactory system of a beetle, based on the emerging model organism $T$. castaneum to create the basis of future studies.

This should be achieved by:

- RNAseq-based genome wide expression analysis to reannotate the computational predicted gene models for OBPs, CSPs, IRs, GRs, ORs, SNMPs, and potential ODEs, to quantify their expression levels in different olfactory, gustatory and non-chemosensory-specific tissues.

- chromosomal localization of these chemosensory relevant genes to identify and support potential recent gene radiations.

- phylogenetic comparison of these genes to homologs of other species with available tissue specific expression data, to evaluate sequence conservation in the context of tissue-specific expression.

- MALDI-TOF-MS based proteome analysis of antennae, to identify relevant OBPs/CSPs.

- scanning electron microscopic characterization of the olfactory sensilla and evaluation of their numbers.

- confocal microscopic characterization and determination of the numbers and projection patterns of chemosensory neurons in palps and antennae by immunohistochemistry, generation of transgenic reporter lines, and backfills.

- immunohistochemically characterization of the antennal lobe and identification of projection neurons to higher brain centers by dye injections. 


\section{Results}

Every chapter within the results starts with a description of:

- the main aim of the particular manuscript in the context of the complete thesis.

- the authors

- my contributions to the respective manuscript.

- the status of the manuscript. 


\subsection{Tissue-specific transcriptomics, chromosomal localization, and phylogeny of chemosensory and odorant binding proteins from the red flour beetle Tribolium castaneum reveal subgroup specificities for olfaction or more general functions}

The aim of this part was to the evidence based reannotation of the computational predicted gene models for odorant binding proteins (OBP) and chemosensory proteins (CSPs) of $T$. castaneum, the chromosomal localization and tissue specific quantification of their expression, as well as the phylogenic comparison with OBPs/CSPs of Drosophila melanogaster and Anopheles gambiae, to evaluate whether conserved OBPs/CSPs show a similar expression profile. Additionally, it should be investigated which OBPs/CSPs are present in the antennae on protein level.

Authors: Stefan Dippel, Georg Oberhofer, Jörg Kahnt, Lizzy Gerischer, Lennart Opitz, Joachim Schachtner, Mario Stanke, Stefan Schütz, Ernst A Wimmer, and Sergio Angeli

Status: Published in BMC Genomics, 2014. 15:1141 (doi: 10.1186/1471-2164-15-1141).

\section{My contributions:}

Drafting and revision of the manuscript.

Figure 1: Chromosomal localization and chemosensory expression profile of Tribolium CSPs and OBPs.

A: Data analysis and visualization (all in silico).

B: Tissue collection, RNA and protein isolation, bioinformatic analysis (building a modified gene set by manual curation of the gene models; analysis of the read-counts and statistical data; analysis of the MALDI-TOF hits), and visualization.

Figure 2: Comparison of expression levels of CSPs and OBPs in male and female antennae, average values based on two male and three female antennal samples. Same as for Figure 1B without proteomics.

Figure 3: Mid-point rooted phylogenetic tree of CSP (A) and OBP (B) sequences.

A: Data analysis (see figure 1B without proteomics and in silico collection of $D$. melanogaster and $A n$. gambiae data), analysis (calculation of the phylogenetic tree and the relative expression levels), and visualization.

B:see A, except for the calculation of the phylogenetic tree.

Figure 4: Multiple alignments of CSPs (A), OBPs (B), and C-OBPs including TcOBP7D (C) made with ClustalW2 and visualized with Jalview 2.0.1. Data acquisition, analysis and visualization (all in silico).

Additional file 1: Table S1: Same as for Figur 1B. Also collation or analysis of data for columns 1-17, and partially of column 18-42).

Additional file 2: Figure S1: Same as for Figure 3.

Additional file 3: Table S2: Tissue collection and protein extraction, analysis (assignment of corresponding gene).

Additional files 4 - 7: Figures S2 - S5: Same as for Figure 1B without proteomics. 


\title{
Tissue-specific transcriptomics, chromosomal localization, and phylogeny of chemosensory and odorant binding proteins from the red flour beetle Tribolium castaneum reveal subgroup specificities for olfaction or more general functions
}

Stefan Dippel ${ }^{1,2 \dagger}$, Georg Oberhofer ${ }^{1 \dagger}$, Jörg Kahnt ${ }^{3}$, Lizzy Gerischer ${ }^{4}$, Lennart Opitz ${ }^{5}$, Joachim Schachtner ${ }^{6}$, Mario Stanke ${ }^{4}$, Stefan Schütz ${ }^{2}$, Ernst A Wimmer ${ }^{1 *}$ and Sergio Angeli ${ }^{2,7}$

\begin{abstract}
Background: Chemoreception is based on the senses of smell and taste that are crucial for animals to find new food sources, shelter, and mates. The initial step in olfaction involves the translocation of odorants from the periphery through the aqueous lymph of the olfactory sensilla to the odorant receptors most likely by chemosensory proteins (CSPS) or odorant binding proteins (OBPS).

Results: To better understand the roles of CSPS and OBPs in a coleopteran pest species, the red flour beetle Tribolium castaneum (Coleoptera, Tenebrionidae), we performed transcriptome analyses of male and female antennae, heads, mouthparts, legs, and bodies, which revealed that all 20 CSPs and 49 of the 50 previously annotated OBPs are transcribed. Only six of the 20 CSP are significantly transcriptionally enriched in the main chemosensory tissues (antenna and/or mouthparts), whereas of the OBPs all eight members of the antenna binding proteins II (ABPII) subgroup, 18 of the 20 classic OBP subgroup, the C+OBP, and only five of the 21 C-OBPs show increased chemosensory tissue expression. By MALDI-TOF-TOF MS protein fingerprinting, we confirmed three CSPS, four ABPIls, three classic OBPs, and four C-OBPs in the antennae.

Conclusions: Most of the classic OBPs and all ABPlls are likely involved in chemoreception. A few are also present in other tissues such as odoriferous glands and testes and may be involved in release or transfer of chemical signals. The majority of the CSPS as well as the C-OBPS are not enriched in antennae or mouthparts, suggesting a more general role in the transport of hydrophobic molecules.
\end{abstract}

Keywords: Chemosensory protein (CSP), Gustation, Odorant binding protein (OBP), Olfaction, Proteome, Transcriptome, Tribolium castaneum

\footnotetext{
* Correspondence: ewimmer@gwdg.de

${ }^{\dagger}$ Equal contributors

'Department of Developmental Biology, Georg-August-University Goettingen,

Johann-Friedrich-Blumenbach-Institute for Zoology and Anthropology,

GZMB, Ernst-Caspari-Haus, Justus-von-Liebig-Weg 11, Goettingen 37077,

Germany

Full list of author information is available at the end of the article
} 


\section{Background}

The red flour beetle Tribolium castaneum (Herbst, Coleoptera, Tenebrionidae) is a secondary pest of stored, dried food products [1]. As a coleopteran model system, it represents the largest insect order, containing many different pests like bark beetles (Dendroctonus ponderosae, Ips typographus), colorado potato beetle (Leptinotarsa decemlineata), pollen beetle (Brassicogethes aeneus) and the Western corn rootworm (Diabrotica virgifera), which cause severe economic and ecological damage. Over the past years, $T$. castaneum turned into a remarkable model organism with plenty of genetic tools such as systemic RNA interference [2,3], forward genetics based on insertional mutagenesis [4], transgene-based mis-expression systems [5,6], as well as a fully annotated genome sequence $[7,8]$. These tools predestine $T$. castaneum as a model system for coleopterans and to investigate findings from the vinegar fly Drosophila melanogaster for their generality in insects.

Odor discrimination is a key process in insect life: from food and host finding to partner recognition, insects rely strongly on odor stimuli. Perception of odorants takes place in the chemosensory (olfactory or gustatory) sensilla and is supposed to be mediated by chemosensory proteins (CSPs) or odorant binding proteins (OBPs) [9-13], followed by detection via odorant receptors (ORs), ionotropic glutamate-like receptors (IRs), or gustatory receptors (GRs) [14]. The olfactory sensilla are hair like structures with the highest density on the antennae. They are housing the dendrites of the odorant receptor neurons and are filled with aqueous lymph. This lymph is secreted by non-neuronal auxiliary cells and contains some CSPs and OBPs [15,16]. The CSPs and OBPs are small (10 to $30 \mathrm{kDa}$ ), globular, and water soluble proteins [17] providing a hydrophobic pocket for ligand binding [18]. The CSPs are characterized by four, conserved cysteine residues forming two disulfide bonds $\left(C_{1}-C_{2}, C_{3}-C_{4}\right)$ [19]. The OBPs - classic OBPs and antennal binding proteins (ABPIIs) [17] have six highly conserved cysteine residues forming three interlocking disulfide bonds $\left(\mathrm{C}_{1}-\mathrm{C}_{3}, \mathrm{C}_{2}-\mathrm{C}_{5}, \mathrm{C}_{4}-\mathrm{C}_{6}\right)$ between six $\alpha$-helices, conferring a high stability to these proteins $[18,20]$. The $\mathrm{C}$-OBPs seem to be derived from classic OBPs and are lacking the $\mathrm{C}_{2}-\mathrm{C}_{5}$ disulfide bridge [17,21-23].

It is believed that hydrophobic semiochemicals interact first with CSPs or OBPs to get shuttled through the aqueous sensillar lymph and to finally reach and activate ORs [14]. Besides evidence that CSPs are involved in chemoreception of the alfalfa plant bug Adelphocoris lineolatus and the Japanese carpenter ant Camponotus japonicus [24,25] and their presence in the antennae of various species $[10,13,26-29]$, there are no functional experiments conducting a role in chemo-sensation. In contrast, the involvement of OBPs in olfaction has been verified by several functional studies: experiments conducted with moth pheromone receptors in heterologous expression systems [30-32] or in vivo using the Drosophila melanogaster "empty neuron system" [33,34] revealed that the presence of the corresponding OBP (pheromone binding protein, $\mathrm{PBP}$ ) increases the sensitivity to the pheromone by 2 to 3 orders of magnitude (reviewed in [14]). Additionally, D. melanogaster mutants for the OBP Lush [35], allelic variation of different OBPs in D. melanogaster [36] and of an OBP in the fire ant Solenopsis invicta [37], as well as several RNAi based experiments in D. melanogaster and mosquitoes [38-40] showed that OBPs are important for the correct and highly sensitive reception of different semiochemicals in these insects, but might not be absolutely essential [41] However, expression analysis of different insects have revealed that CSPs and OBPs are not restricted to the main chemosensory tissues [42-48] but are also involved in other tasks, e.g. the release of semiochemicals [49], mating [50], embryogenesis [51], immune-response [52], and regeneration [53]. Moreover, olfactory based systems [54] such as OBP coupled biosensors might improve pest and plant disease monitoring [55,56], risk assessment [57], or prevent infestation by camouflaging or repelling [58,59]. This could offer novel eco-friendly and cost effective ways to combat the fast adaption of Tribolium against several insecticides and respective resistance development $[60,61]$ and thus improve the protection of stored agricultural products [62] against migrating beetles [63].

In this study we use tissue-specific transcriptomics to improve the genome annotation of the $T$. castaneum CSPs and OBPs and to determine their expression profile. We place these data into a phylogenetic context in order to get better insights into their potential functions with a comparative evolutionary perspective.

\section{Methods \\ Tribolium rearing}

T. castaneum strain San Bernardino (Herbst, 1797; Insecta, Coleoptera, Tenebrionidae), was reared on organic wheat flour supplemented with $5 \%$ yeast powder at $28^{\circ} \mathrm{C}$ and $40 \%$ relative humidity under constant light. The Beetles were collected from different breeding boxes varying in age (up to three month) and culture density.

\section{RNA isolation and sequencing}

From the sex separated and age pooled animals about 1000 antennae, 600 legs, 150 mouthparts (as piece of the head capsule anterior of the antennae), 50 heads (the whole head capsule excluding the antennae) and 20 bodies (excluding head and legs) were manually dissected and immediately transferred to ice cold RNA lysis buffer (Zymo Research, Irvine, USA). For larval tissues about 
100 heads and 50 bodies of unsexed last instar larvae were collected. Total RNA was isolated using the ZR Tissue \& Insect RNA Micro Prep Kit (Zymo Research, Freiburg, Germany) following the manufacturer's protocol. The Library preparation for RNA-Seq was performed using the TruSeq RNA Sample Preparation Kit (Illumina, San Diego, USA) starting from $300 \mathrm{ng}$ of total RNA. Accurate quantification of cDNA libraries was performed by using the QuantiFluor ${ }^{\mathrm{TN}}$ dsDNA System (Promega, Fitchburg, USA). The size range of final cDNA libraries (280 bp) was determined applying the DNA 1000 chip on the Bioanalyzer 2100 (Agilent, Santa Clara, USA). cDNA libraries were amplified and sequenced using the cBot and HiSeq2000 from Illumina (paired end; $2 \times 100 \mathrm{bp}$ ). Sequence images were transformed with Illumina software BaseCaller to bcl files, which were demultiplexed to fastq files with CASAVA v1.8.2 (Illumina). Quality check was done via fastqc (v. 0.10.0, Babraham Institute, Cambridge, UK).

\section{OBP and CSP re-annotation, SNP calling and mapping}

The obtained fastq formatted Illumina reads were mapped to the Tribolium castaneum 3.0 official gene set using bowtie2 [64] with the "very-sensitive" presetting. The previously published CSP and OBP sequences [7,17,21,65] were identified in this gene set with blastp [66] implemeted in bioperl [67]. Samtools mpileup (v0.1.18) [68] was used to check the RNAseq data for SNPs and indels. In a genome independent approach a de novo assembly was built. Quality filtering was performed with the NGSQC Toolkit (v2.3.1) [69] in three steps: 1) removal of reads containing ambiguous bases with AmbiguityFiltering.pl, all settings default; 2) trimming of bad quality bases from 3' ends with TrimmingReads.pl, -q 28 -n 60;3) removal of bad quality reads with IlluQC_PRLL.pl, N 5 -1 90 -s 24 . Before the assembly the reads were digitally normalized using the normalize_by_kmer_coverage.pl script from trinity (release2013_08_14) [70] with these settings: --max_cov 50 -pairs_together. The assembly was performed with Trinity.pl, all settings default. Translations of open reading frames were extracted with transcripts_to_best_scoring_ORFs.pl. The preliminary reannotation of the whole T. castaneum gene set (au3) was generated by the gene finder AUGUSTUS [71]. Alignments of RNA-Seq reads from libraries from several tissues, stages and conditions (e.g. embryo, larva head, larva body, early and late stage pupa, adult antenna, leg, head, body, stink glands, ovary) were incorporated. These data were produced mainly by the iBeetle consortium [72] and a separate publication is in preparation describing the reannotation of the whole gene set of $T$. castaneum based on these RNA-Seq data, which can be viewed in a respective genome browser [73]. It contains a track with the au3 gene models as well as RNA-Seq coverage tracks of different stages including the data collected for this study. In total 1,624,983,955 reads were mapped against the genome with the alignment tool BLAT [74]. The read alignments were filtered, so that only alignments of reads that mapped uniquely to the genome and that showed a percent identity of at least $93 \%$ were kept. Paired reads were required to be aligned in the correct orientation and with a maximal genomic distance of 500,000 base pairs. Intron evidence was collected based on reads with a spliced alignment against the genome and evidence for transcription is taken from RNA-Seq covered regions. In an iterative process, the SNP calling, the de novo assembly, and the au2 gene set were used to manually curate the OBP and CSP sequences based on previously published annotations $[7,17,21,65]$. The corresponding au3 gene models were replaced with these new candidate sequences and the resulting modified au3 gene set was used to remap the RNAseq data with bowtie 2 using the 'verysensitive' presetting. Finally all sequences were searched for signal peptides using the SignalP4.1 server [75] and browsed for conserved functional domains [76].

\section{Tribolium castaneum expression profiling}

The mapped reads of the re-annotated OBPs and CSPs in the particular tissue or sex sample were counted with samtools [68]. To normalize the count numbers RPKM values were calculated and plotted as $\log 2[\mathrm{RPKM}+1]$ (Additional file 1: Table S1). The values were visualized using the matrix2png interface (version 1.2.1; [77]) and the figures were composed with inkscape [78]. Male and female reads from the sequenced tissues were pooled and considered as biological replicates. Statistical analysis of the data was performed in $R$ [79] using the DESeq package (version1.12.0) [80] from bioconductor [81]. All sequenced tissues were compared to body as reference. Significant differentially expressed genes (false discovery rate $<0.05)$ are marked with asterisks. For the intersex comparison the two male and three female replicates of antenna were treated the same way.

\section{Phylogenetic analysis and interspecies comparison}

We compared our sequences on protein level with data from D. melanogaster and the malaria mosquito Anopheles gambiae obtained from Vieira and Rozas 2011 [17]. After subtraction of the signal peptide (SignalP4.1) [75], the sequences were aligned using MAFFT v7.040b [82] as described [17] and the tree was constructed using RAxML version 7.8.6. [83] with the LG substitution model in the case of the CSPs or the VT substitution model for the OBPs and GAMMA correction. Node support was assessed with 100 rapid bootstrap replicates. The relative expression levels were calculated as $\log 2$ fold changes of antenna/body and palp (mouthpart)/ body. For $T$. castaneum, $\log 2 \mathrm{FC}$ data from inner species 
comparison were used. The D. melanogaster data set was downloaded from EMBL gene expression atlas [84] originally published in Farhadian et al. 2012 [43] and the An. gambiae data were obtained from Pitts et al. 2011 [42]. The phylogenetic tree was visualized by iTOL [85] and descriptions were added using inkscape [78]. Since the absolute expression levels of the different candidates are lost in the depiction of the fold changes, we provide them in Additional file 2: Figure S1. Please note that the methods used to obtain the different expression data (RNA-seq and microarray) are not directly comparable. Therefore, Additional file 2: Figure S1 can just give an impression on more or less abundant transcripts.

\section{Cloning of selected OBPs and CSPs open reading frames}

Manually separated heads were ground in liquid nitrogen, and total RNA was extracted using the TRIZOL reagent (life technologies, Carlsbad, USA). Messenger RNA was purified with the Dynabeads purification kit (life technologies, Carlsbad, USA) and cDNA was synthesized using the Super-Script first-strand synthesis system (life technologies, Carlsbad, USA). Hotstart Taq DNA polymerase (Qiagen, Venlo, Netherlands) was used to amplify individual transcripts. Finally the products were cloned into PCR2.1 vector (life technologies, Carlsbad, USA) and verified by sequencing. Most primers were designed to bind within the UTRs to not bias start and stop codons and are summarized in Additional file 1: Table S1.

\section{MALDI-TOF MS}

For identification of OBPs and CSPs on protein level, about 400 antennae per sample were manually separated and homogenized in $200 \mu \mathrm{l}$ milliQ water containing $0.1 \%$ trifluoroacetic acid (Sigma-Aldrich, St. Louis, USA) with a tube fitting pestle. To get rid of the debris, the samples were centrifuged and $150 \mu$ l supernatant was used further. To break down the secondary structure, the disulfide bridges were reduced and simultaneously the cysteine-derived thiol groups alkylated with $10 \mu \mathrm{l}$ $100 \mathrm{mM}$ tris (2-carboxyethyl) phosphine hydrochloride (Chemos GmbH, Regenstauf, Germany), $10 \mu \mathrm{l} 200 \mathrm{mM}$ 2-vinylpyridine (in 30\% acetonitrile, Sigma-Aldrich) and $26 \mu \mathrm{l}$ M guanidine hydrochloride (Sigma-Aldrich) for $8 \mathrm{~min}$ at $35^{\circ} \mathrm{C}$, followed by additional incubation for $30 \mathrm{~min}$. at $\mathrm{pH} 8$ after adding $11 \mu \mathrm{l} 1 \mathrm{M}$ ammonium bicarbonate (Sigma-Aldrich). The sample was loaded on a VIVASPIN 500 VS011 Ultrafiltration unit (5000 MWCO, Sartorius, Goettingen, Germany) and centrifuged for $10 \mathrm{~min}$ followed by two washing steps with $200 \mu \mathrm{lmilliQ}$. For storage at $-20^{\circ} \mathrm{C}$ over night the remaining $50 \mu \mathrm{l}$ sample was mixed with $100 \mu \mathrm{l}$ milliQ and $50 \mu \mathrm{l}$ acetonitrile. After $30 \mathrm{~min}$ of centrifugation, $100 \mu \mathrm{lmilliQ}$, $50 \mu \mathrm{l} 50 \mathrm{mM}$ Ammonium bicarbonate and $20 \mu \mathrm{l}$ acetonitrile were added and debris was removed by additional centrifugation. Digestion took place in the remaining volume over the membrane by adding $0.11 \mu \mathrm{g}$ sequencinggrade modified trypsin (Promega, Fitchburg, USA) and the resulting peptides were eluted. The mixture was analyzed by nanoLC (PepMap100 C-18 RP nanocolumn and UltiMate 3000 liquid chromatography system; Dionex, Sunnyvale, USA) and automated MSMS (4800 Proteomics Analyzer MDS, AB Sciex, Framingham, USA). MSMS data were searched against the au2 gene set (http://bioinf. uni-greifswald.de/gb2/gbrowse/tcas4/) using Mascot embedded into GPS explorer software (AB Sciex). Identified proteins, their scores, and Pfam predictions are provided in Additional file 3: Table S2.

\section{Results and discussion}

Re-annotation and re-naming of Tribolium CSPs and OBPs In the past, several authors published sequences of Tribolium CSPs and OBPs based on computational predictions $[7,17,21,65]$ resulting in different conflicting annotations and designation. We revised the originally described 20 CSPs and 50 OBPs using transcriptome analysis of different tissues including antennae and mouthparts. Subsequently we applied a new nomenclature to prevent confusion and to provide a unique and distinguishable nomenclature following the one used for Drosophila OBPs [22]. We used the prefaces CSP, and OBP to reflect the fact that a gene is a member of one of these protein families. This is followed by a number reflecting the chromosomal location and a letter that conveys its relative position on the chromosome (Figure 1A). Thus, the new name OBP9B refers to the second OBP on the ninth chromosome. A comparative list putting all previous names in relation can be found in the Additional file 1: Table S1.

We detected reads corresponding to all previously described Tribolium CSPs and OBPs except TcOBP2A. Accordingly we confirmed or corrected the predicted open reading frames of all 20 CSPs and 49 OBPs. In case of low abundant transcripts with poor read coverage in our samples we used additional data obtained from embryo and pupa to support the re-annotation (iBeetle genome browser) [73]. The comparison of the latest genome based annotation [17] with our transcriptome based reannotation revealed differences mainly based on wrongly predicted intron-exon boundaries. The identified discrepancies to previous annotations in the OBPs and one CSP did not cause severe differences in the phylogenetic relationship. However, they partially affect in addition to the intron-exon boundaries also the start or stop codons, which could impair cloning efforts for further investigations. In addition, wrong indels can cause differences in three-dimensional modeling of OBPs and by this also affect predictions in respect to potential ligands. Therefore, we point out clearly which annotations of previously identified OBPs and CSPs needed to be changed: Due 

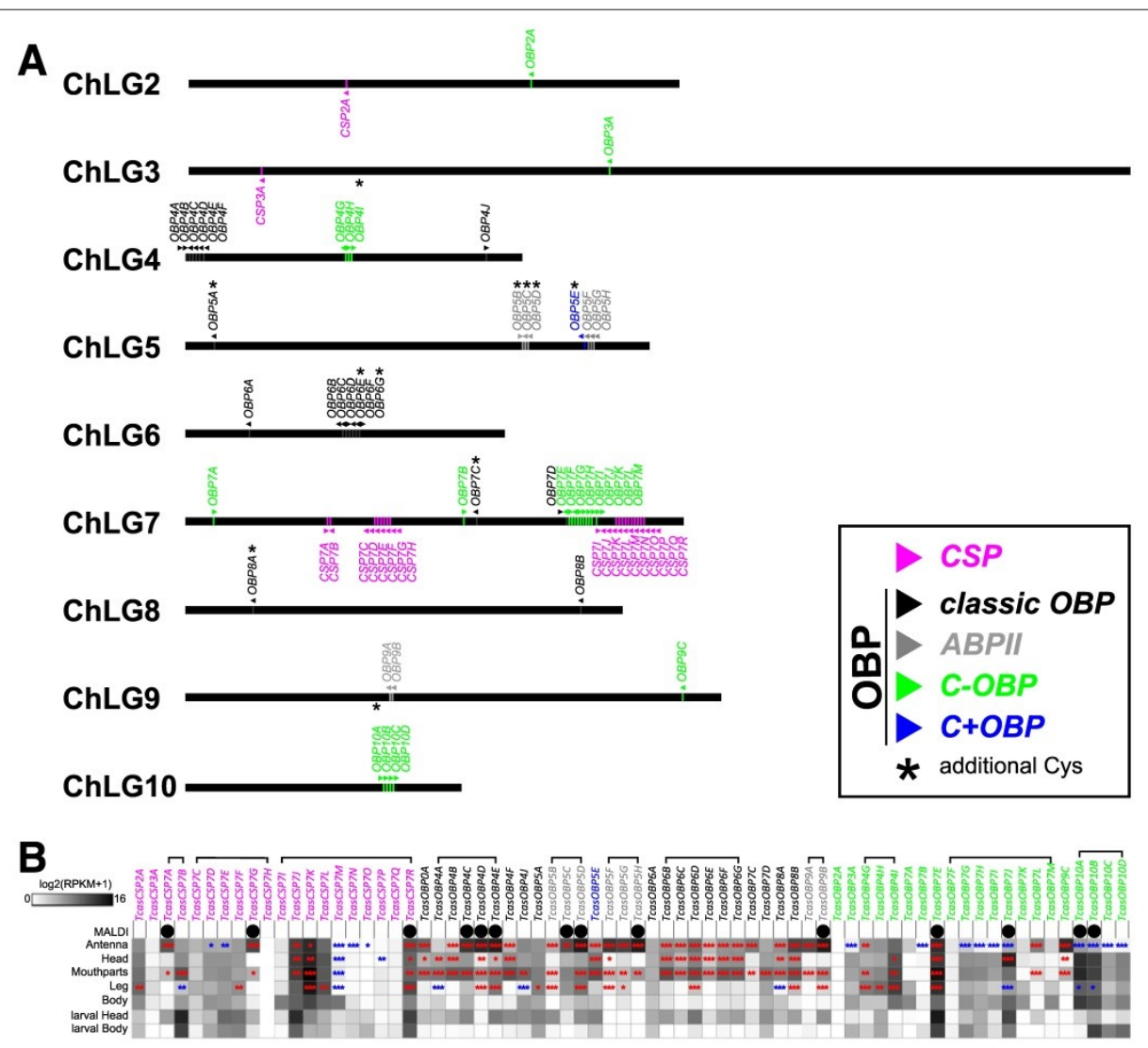

Figure 1 Chromosomal localization and chemosensory expression profile of Tribolium CSPs and OBPs. (A) Chromosomal localization of CSPs (magenta), classic OBPs (black), ABPII (grey), C-OBPs (green) and C + OBP (blue), based on Georgia GA-2 strain genome assembly 3.0 [7]. The arrowheads indicate the orientation of the genes from $5^{\prime} 3^{\prime}$. Genes encoding for more than the six highly conserved cysteines (or four in case of C-OBP) are labeled with an asterisk (*). (B) Heatmap showing the absolute expression level of the OBPs/CSPs as log2 (RPKM + 1) in different tissues (adult antennae, head (missing antennae but including mouth parts), mouthparts, legs, body, as well as larval head and body). The candidates are blotted according to their chromosomal localization, horizontal brackets above indicate clustering in the genome. A black dot in the first row labeled 'MALDI' indicates that at least one unique tryptic fragment of the particular candidate was identified in an antennae sample on protein level. The expression levels are represented by a log2 greyscale with high expression levels $\left(2^{16}\right.$ RPKM) labelled black. The asterisks mark statistically significant differtially expressed genes compared to body. The red asterisks represent up- and the blue down-regulation (p-values are $\left.{ }^{*}<0.05 ;{ }^{* *}<0.01 ;{ }^{* *}<0.001\right)$.

to an unpredicted intron in the 5'UTRs of TcCSP2A, TcOBP0A and TcOBP5F the start codons had to be changed. In case of TcOBP5E, TcOBP6A, TcOBP6E, TcOBP6F, TcOBP8A, TcOBP7G and TcOBP7H wrong or un-predicted introns led to insertions or deletions not affecting the highly conserved cysteine pattern. In TcOBP4A, TcOBP4C, TcOBP4E, TcOBP4F, TcOBP5C, TcOBP5D and TcOBP5H an incorrect last intron caused differences in the $\mathrm{C}$-terminal region, that was supposed to be involved in ligand binding and release $[86,87]$. Additionally we were able to revive previously wrongly annotated pseudogenes. Formerly termed OBP49P is an intact gene with some characteristics of an OBP and is now called TcOBP7C. OBP50P [17] turned out to correspond to the already described OBP-C08 [7] and is now called TcOBP7K. The originally termed CSP21P, which is derived from a duplication of the first exon of TcCSP7G, is expressed at low levels and now called TcCSP7H, which either represents an expressed pseudogene as observed in Nasonia [88] or is a truncated CSP. Because of its high sequence similarity to TcCSP7G we were only able to map unique reads to the 3'UTR. Therefore, expression of TcCSP7H is not indicated in our figures. To confirm the new annotation we checked the resulting protein sequences for common characteristics of CSP- or OBPlike signal peptide, conserved domains, size and cysteine composition [89] and we cloned the whole open reading frame of 33 of the 70 candidates from cDNA. All newly annotated proteins contain a predicted $\mathrm{N}$ terminal signalpeptide with an length of 15 to 22 AA (SignalP 4.1 [75]). According to the conserved domain database [76], all reannotated CSPs and OBPs (except the C+OBP, OBP5E) are members of the OS-D respectively PBP_GOBP superfamily (Additional file 1: Table S1). 
The re-annotated CSPs of Tribolium have an average size of 128 AA (110 AA mature CSP), they range from the smallest, TcCSP7C, with just 99 AA to the longest, TcCSP7E, with 251 AA. On average, Tribolium CSPs are within the size range of other species [12]. The cysteine formula of the Tribolium CSPs follows the highly conserved pattern with four cysteines arranged by an exact spacing of $\mathrm{C}_{1 \mathrm{X}_{6}} \mathrm{C} 2 \mathrm{X}_{18} \mathrm{C} 3 \mathrm{X}_{2} \mathrm{C} 4$ [17]. The only exception is the pseudo/truncated gene $\mathrm{TcCSP} 7 \mathrm{H}$ that stops after $\mathrm{C} 2$.

The OBPs are slightly longer and vary from 106 up to 320 AA with an average size of 143 AA (125 for the mature OBP), which is similar to other insects such as D. melanogaster (117 up to 245 AA) or An. gambiae (107 up to 356 AA [17]). Most of the T. castaneum classical OBPs (including ABPIIs) show a conserved cysteine pattern $\left(\mathrm{C}_{1} \mathrm{X}_{24-29} \mathrm{C} 2 \mathrm{X}_{3} \mathrm{C} 3 \mathrm{X}_{22-43} \mathrm{C} 4 \mathrm{X}_{8-10} \mathrm{C} 5 \mathrm{X}_{8} \mathrm{C} 6\right)$ comparable to D. melanogaster [22] and other insects. The only exceptions are the C+OBP (TcOBP5E, $241 \mathrm{AA}$ ) and the non-clustered OBPs TcOBP5A, TcOBP7C, and TcOBP8B (Figure 1A), which differ in $C$ spacing, are of unusual length (TcOBP5E, 241AA; TcOBP5A, 176 AA; TcOBP7C 320 AA; TcOBP8B, $200 \mathrm{AA}$ ), and are phylogenetically close to $\mathrm{C}+\mathrm{OBP}$. The one typical $\mathrm{C}+\mathrm{OBP}$, TcOBP5E, has an expanded $\mathrm{N}$ and $\mathrm{C}$ terminus containing six additional cysteines, whereas TcOBP7C has an expanded $\mathrm{N}$ terminus, TcOBP8B extra AAs between $\mathrm{C} 1$ and $\mathrm{C} 2$, TcOBP5A between $\mathrm{C} 1$ and $\mathrm{C} 2$ plus between $\mathrm{C} 4$ and $\mathrm{C} 5$. TcOBP5A, TcOBP8B, and TcOBP5E have extra cysteines 17-19 AA before $\mathrm{C} 1$ and nine AA after C6. The OBPs TcOBP5B, TcOBP5C, TcOBP5D, TcOBP6E, TcOBP6G, TcOBP7C, and TcOBP8A contain also at least one additional cysteine. TcOBP6G and the ABPIIs (TcOBP5B, TcOBP5C, TcOBP5D) have a conserved additional cysteine seven AA after C3. Despite the increased amount of cysteines only TcOBP5E carries the typical proline residue following C6 (C6b) [90,91]. The C-OBPs show a conserved cysteine pattern, with only four cysteines, lacking $\mathrm{C} 2$ and $\mathrm{C} 5$ (C1X $\left._{18-30} \mathrm{C} 3 \mathrm{X}_{37-39} \mathrm{C} 4 \mathrm{X}_{16-17} \mathrm{C} 6\right)$. We can conclude that all re-annotated CSPs (except CSP7H) and OBPs fulfill the rigid criteria previously defined based on other species $[22,89]$.

\section{Expression profile of the CSPs and OBPs in Tribolium castaneum}

As several CSPs and OBPs are supposed to be involved in olfaction, we comparatively analyzed the expression of these genes in the main chemosensory tissues antennae and mouthparts (here defined as the piece of the head capsule anterior to the antennae) plus in heads (the whole head capsule excluding the antennae), legs, and bodies (excluding head and legs) of males or females, respectively. To get some first insights into expression differences between larval and adult stages, we also sequenced heads including antennae and bodies (without head) of last instar larvae. The results as $\log 2$ RPKM are represented as heat-map in Figure 1B.

The expression of the majority of the CSPs is detected in a wide variety of tissues. Transcripts of only five of the 20 CSPs are significantly enriched in antennae (TcCSP7A, TcCSP7G, TcCSP7J, TcCSP7K, and TcCSP7R) and six in the mouthparts (TcCSP7A, TcCSP7B, TcCSP7G, TcCSP7J, $T c C S P 7 K$, and $T c C S P 7 R)$. However, only the expression of $T c$ CSP7A and TcCSP7G is restricted to the main olfactory tissue. TcCSP7I and TcCSP7M are exclusively expressed in the body. Six of the CSPs showed no or only poor expression in our tissue samples, however, we found them expressed at other developmental stages by searching the iBeetle genome browser [73]. TcCSP7P and TcCSP7Q are expressed in embryo and pupa, TcCSP3A mainly in embryo, TcCSP7N in embryo and larva, and TcCSP7O only in larva.

In contrast to the CSPs, the expression of the OBPs is more restricted to the main chemosensory tissues (antennae and mouthparts). All eight ABPIIs are highly expressed in the antennae. With the exception of TcOBP5C, all of them are also significantly enriched in mouthparts indicating an involvement of this subgroup in chemoreception (Figure 1B). 15 of the 20 classic OBPs are significantly enriched in antennae and mouthparts (Figure 1B, TcOBP0A, TcOBP4B, TcOBP4C, TcOBP4D, TcOBP4E, TcOBP4F, TcOBP6B, TcOBP6C, TcOBP6D, TcOBP6E, TcOBP6F, TcOBP6G, TcOBP7C, TcOBP8A, TcOBP8B), whereas three are enriched only in the mouthparts (TcOBP4A, TcOBP4J, TcOBP7D). Only three of the classic OBPs are evenly expressed in all tissues. Interestingly, these are the non-clustered ones (TcOBP5A, TcOBP6A, TcOBP7D). The C + OBP (TcOBP5E) is enriched in mouthparts and in antennae. Most of the 21 C-OBPs are expressed in all tissues similar to the majority of the CSPs, only five are significantly enriched in antennae and mouthparts compared to body with three of them also highly abundant in head or leg (TcOBP4G, TcOBP4I, TcOBP7E). Thus, there are just two C-OBPs (TcOBP7L and TcOBP9C) most likely exclusively involved in chemosensory processing. Ten C-OBPs are significantly down-regulated in the antennae compared to the body: TcOBP3A, TcOBP7B, TcOBP7G, TcOBP7H, TcOBP7I, TcOBP7J, TcOBP10A, TcOBP10B, TcOBP10C, and TcOBP10D. TcOBP2A expression was not detected at all, TcOBP7F is expressed during metamorphosis and TcOBP7B is mainly active during embryogenesis as well as metamorphosis.

Statistical analysis of the two male and three female antennal samples did not show any significant difference, due to the low abundance of potential candidates and the relative high dispersion of the samples (Figure 2; Additional file 4: Figure S2, Additional file 5: Figure S3, Additional file 6: Figure S4 and Additional file 7: Figure S5). 


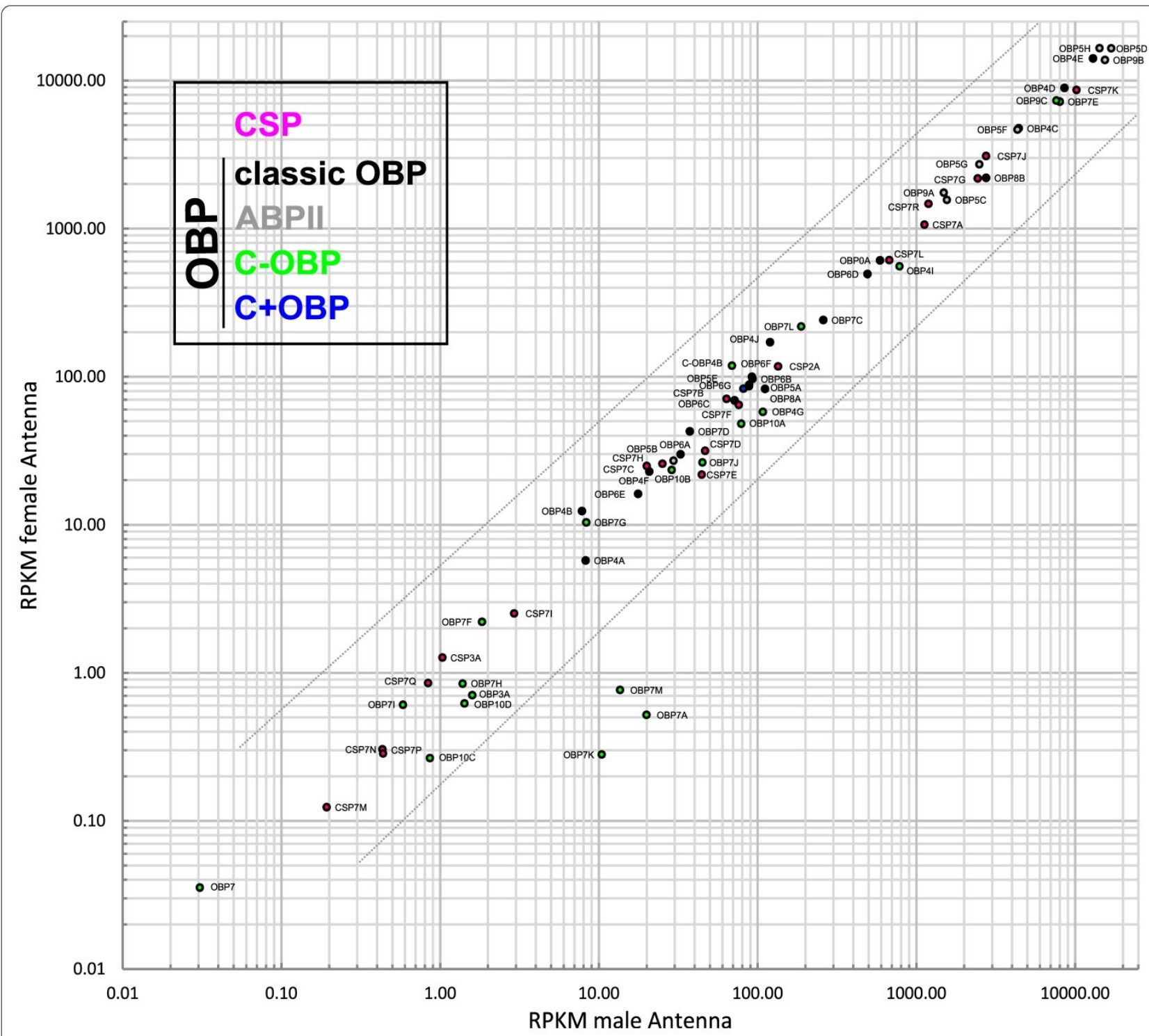

Figure 2 Comparison of expression levels of CSPs and OBPs in male and female antennae, average values based on two male and three female antennal samples. Scatter plot of the RPKM values of the CSPs (in pink) and OBPs (classic in black, ABPII in grey, C-OBP in green, $\mathrm{C}+\mathrm{OBP}$ in blue). The dotted lines represent a fivefold difference.

Nevertheless, TcOBP7A, TcOBP7K, and TcOBP7M were more than five-fold overexpressed in male antennae and could be interesting for further investigation (Figure 2). The fact that we found no major and significant differences between male and female is consistent with anatomical data from the antennal lobe were no sexual dimorphism was found [92], and the antennal morphology from a related species, Tribolium brevicornis, were both sexes are anatomically similar [93].

The comparison of transcriptome data of adult antennae and larval heads revealed differences in the expression of five ABPIIs (TcOBP5C, TcOBP5F, TcOBP5G, $T c \mathrm{OBP} 5 \mathrm{H}$, and $T c \mathrm{OBP} 9 \mathrm{~A})$, one classic OBP (TcOBP4D) and five C-OBPs (TcOBP3A, TcOBP4G, TcOBP4I, TcOBP7I, and $T c \mathrm{OBP} 9 \mathrm{C})$. Most of these transcripts were present in adult antennae or mouthparts but were absent in the larval head (Figure 1B) reflecting the reduced larval olfactory system which also corresponds to the lower amount of expressed odorant receptors previously described (41 in larvae compared to 111 in adults) [94].

In a previous study regarding the stink glands of T. castaneum [95], TcOBP8A, TcOBP6B, TcCSP7P and TcCSP7R were identified to be transcriptionally enriched in the prothoracic glands compared to general anterior abdominal tissue. Interestingly TcOBP8A and TcOBP6B were also enriched in antennae and mouthparts, whereas 
TcCSP7P was only detected in embryo and pupa. It seems that in Tribolium these OBPs are not only involved in reception of odorants/pheromones but also in production or release of such semiochemicals, as postulated for some lepidoptera $[26,49]$.

To relate our transcriptome data to protein detection in the adult antenna, we additionally performed MALDITOF-TOF MS with antennal extracts. We were able to identify fingerprints of 14 of the 70 candidates in antennae on protein level (Figure 1B). Thus, we found at least one tryptic fragment with an ion score above 50 that maps uniquely to the AA sequence of one of the CSPs or OBPs (Additional file 3, Table S2). We identified three highly expressed CSPs (TcCSP7A, TcCSP7G, TcCSP7R), the three highest expressed classic OBPs (TcOBP4C, TcOBP4D, TcOBP4E), four of the highly expressed ABPII subclass (TcOBP5C, TcOBP5D, TcOBP5H, TcOBP9B), and four C-OBPs (TcOBP7E, TcOBP7J, TcOBP10A, TcOBP10B). All identified tryptic fragments belong to genes that are transcribed in the antennae, therefore confirming their expression also on a protein level.

\section{Phylogenetic considerations in respect to the expression of the CSPs and OBPs}

The majority of the 50 OBPs and 20 CSPS of T. castaneum are arranged in clusters like in other insects e.g. D. melanogaster [22], An. gambiae [96], the honey bee Apis mellifera [21] and silk moth Bombyx mori [97]. The CSPs are organized in arrays of two, six and ten genes on the seventh chromosome, only two $(T c C S P 2 A$, TcCSP3A) are non-clustered and located on chromosome 2 and 3. Most of the classic OBPs are arranged in two large arrays on chromosome 4 and 6, only six are interspersed (TcOBP5A, TcOBP6A, TcOBP7C, TcOBP7D, TcOBP8A and TcOBP8B). Six of the eight ABPIIs are located on chromosome 5 with three genes per cluster, the remaining two are close together on Chromosome 9. Nine of the $21 \mathrm{C}$-OBPs are located in a cluster on chromosome 7 , close to the interspersed classic OBP TcOBP7D, that is phylogenetically the closest relative classic OBP to all C-OBPs. Additional three C-OBPs form a cluster on chromosome 4 , four on chromosome 10 and the remaining $5 \mathrm{C}$-OBPs are interspersed on chromosome 2, 7 and 9. The only $\mathrm{C}+\mathrm{OBP}$ (TcOBP5E) is located next to the second ABPII cluster on chromosome 5 , but is phylogenetically unrelated to this group. All other OBPs carrying an additional cysteine are randomly distributed over the genome. The presence of clusters of phylogenetically related genes in all investigated insects can be explained by their origin from gene duplication events within the respective lineage but the fact that the clusters are conserved within different Drosophilidae indicates some constraints that stabilize the clusters [98]. One possible explanation for the maintaining of the clusters is the sharing of regulative elements [99], however, our expression data do not support this theory since genes from the same cluster (Figure 1B, indicated by horizontal brackets) show partially unrelated expression. Most likely more sophisticated methods are needed to understand the complex interplay of regulative elements within a cluster as recently shown for regulatory elements of odorant receptors in Drosophila [100] and their distribution within clusters. An interspecies comparison between T. castaneum, D. melanogaster, and An. gambiae regarding the expression level in a phylogenetic context revealed that some expression features found in T. castaneum are conserved between these species. The majority of the CSPs of all species are expressed in all tissues (Figure 3A). The classic OBPs in the branch holding genes of all three species are mainly enriched in antennae and/or mouthparts (Figure 3B). Only three - namely DmOBP22a, DmOBP56f, DmOBP51a - are clearly underrepresented in the main chemosensory tissues. Also the antennal expression of $T$. castaneum ABPIIs is consistent in the other species (Figure 3B). All members of this subgroup except AgOBP18 are enriched in antennae and the highest expressed OBPs within each species belong to this group.

The comparison of the C-OBPs of $T$. castaneum, An. gambiae, and D. melanogaster revealed that they are polyphyletic (Figure 3B) as previously shown by Vieira and Rozas [17]. The phylogenetic analysis as well as the chromosomal clustering indicates that in $T$. castaneum this large expanded group is together with the classic OBP TcOBP7D most likely derived from a common ancestor. This is similar to the situation in A. mellifera, where a monophyletic group of C-OBPs (AmOBP14 to $A m \mathrm{OBP} 21)$ clusters together with the classic OBP AmOBP13 both on the genomic localization and on the phylogenetic level [21]. However, even though the C-OBPs of different species are polyphyletic in their origin, they are in general highly and equally expressed in all tissues indicating a broad function. The loss of a disulfide bridge might increase their binding flexibility to serve different binding tasks $[10,20,101]$. The C-OBPs (21 in T. castaneum, four in D. melanogaster) actually represent similarly to the CSPs (20 in T. castaneum, four in $D$. melanogaster, eight in An. gambiae) a large expansion in T. castaneum and are mostly not antennae- or mouthpartspecifically expressed. Therefore, these proteins might not be mainly involved in chemosensory detection but might have additional roles such as detoxification which has been discussed for D. sechelia [102].

\section{Conclusion}

Our T. castaneum expression analysis revealed expression of most CSPs and C-OBPs in various body parts, whereas expression of classical OBPs and ABPIIs is mainly restricted to the antennae and mouthparts. These data are 


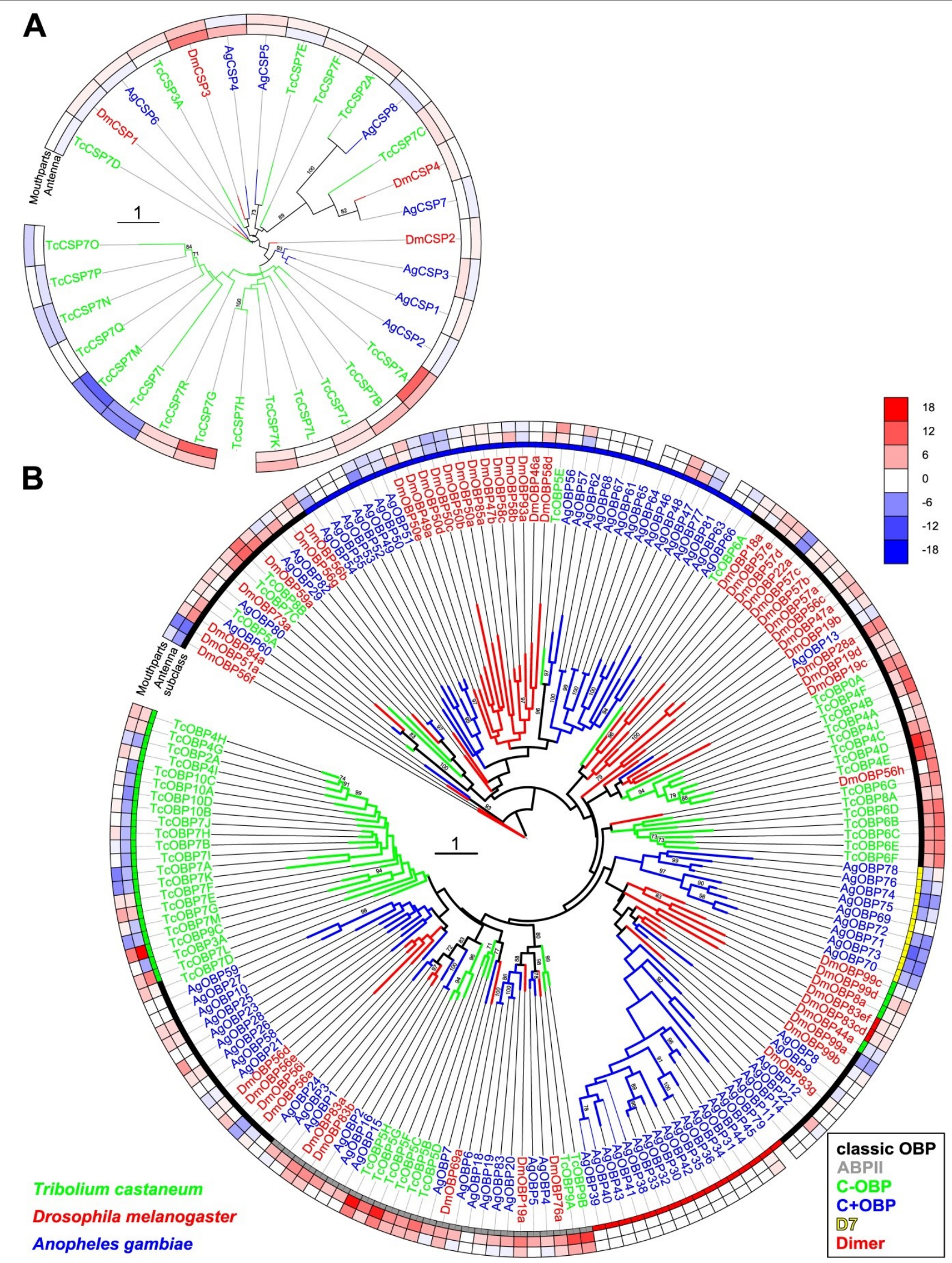

Figure 3 (See legend on next page.) 
(See figure on previous page.)

Figure 3 Mid-point rooted phylogenetic tree of CSP (A) and OBP (B) sequences from Tribolium castaneum (green branches), Drosophila melanogaster (red branches), and Anopheles gambiae (blue branches). Outer rings represent the expression in antennae and 'mouthparts' (Tribolium: palps, mandible, labrum and labium; Drosophila: palp and proboscis; Anopheles: maxillary palp) as log2 fold change compared to body corresponding to the scale in the right middle. The scale bars within the trees represent 1 amino acid substitution per site. Inner ring in B indicates the phylogenetic subclass (classic in black, ABPII in grey, C-OBP in green, C + OBP in blue, D7 in yellow, Dimer in red). Numbers on branches show values of 100 times replication bootstrap analysis higher than 70 .

consistent with previous observations in different insects [48], like A. meliffera [21,65], An. gambiae [42], B. mori [103] and the large black chafer Holotrichia parallela [104]. Systematic OBP knockdowns in D. melanogaster show their necessity for correct olfactory behavioral responses and indicate a combinatorial OBP-dependent odorant recognition [38]. Our comparative expression data suggest that within the classic OBPs, especially the ABPII subgroup has a specific role in olfaction, since all members of T. castaneum, An. gambiae, and D. melanogaster are highly expressed and enriched in the antennae. Moreover, this group contains some of the most prominent OBPs such as D. melanogaster LUSH involved in pheromone detection [35,41], An. gambiae AgOBP4 that forms

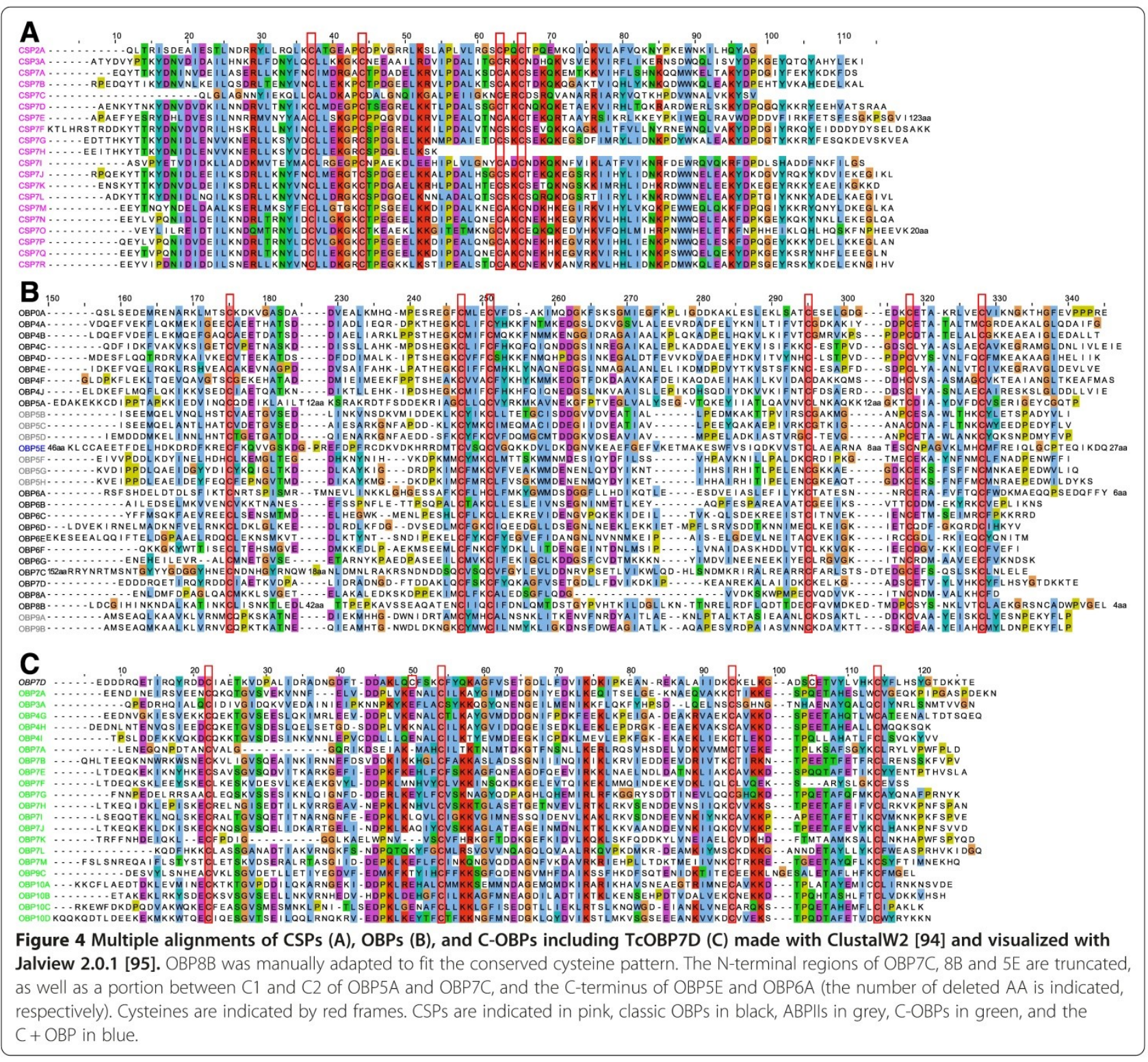


cooperative heteromers with other OBPs [105], and $A g \mathrm{OBP} 1$ that mediates indole detection to find blood meals [39] and is co-expressed with other ABPIIs (AgOBP3, AgOBP4, AgOBP19) [106].

Most OBPs of $T$. castaneum are arranged in clusters in the genome. The only exceptions are TcOBP5A, TcOBP6A, TcOBP7C, TcOBP7D, TcOBP8A, and TcOBP8B. Intriguingly three of them (TcOBP5A, TcOBP6A, TcOBP7D) show an atypical, ubiquitous expression and four differ massively from the average size of 143 AA: TcOBP8A 106 AA; TcOBP5A 176 AA, TcOBP8B 200 AA, and TcOBP7C 320 AA (Figure 4). Interestingly, a bootstrap value of 100 indicates orthology of TcOBP5A with a single widely expressed OBP in An. gambiae (AgOBP80) and D. melanogaster (DmOBP73a) (Figure 3), which seem to have also single orthologues in B. mori, the pea aphid Acyrthosiphon pisum, and the body louse Pediculus humanus [17].

In contrast to the ABPIIs and classic OBPs, the CSPs and $\mathrm{C}$-OBPs show a more broad expression indicating a more versatile function in transport of hydrophobic chemicals involved in various processes. In $T$. castaneum, several functions besides semiochemical reception are implicated: TcCSP7P and TcCSP7R are highly enriched in odoriferous glands [95] and may be involved in the secretion of semiochemicals or defensive products; $T c \mathrm{OBP} 10 \mathrm{~B}$ and $T c \mathrm{CSP} 7 \mathrm{D}$ are up-regulated after cry toxin exposure [107] indicating a function in detoxification or the innate immune system of $T$. castaneum. TcOBP7F is transferred during copulation via seminal fluids [108] similar to the yellow fever mosquito Aedes aegyti [109] to potentially mark fertilized eggs, as also described for Helicoverpa moths [50]. Many more functions of CSPs in insects have sporadically been described, such as involvement in limb regeneration in the American cockroach Periplaneta americana [53], presence in the female reproductive organs of the migratory locust Locusta migratoria [110], involvement in embryonic integument formation in A. mellifera [51], response to an insecticide in the silverleaf whitefly Bemisia tabaci [111] and B. mori [112], detergent like function in the proboscis of two Helicoverpa species [113]. Taken together, some CSPs seem to participate in chemoreception, however, most of them might have more general functions involved in the release of semiochemicals [26], development [51], reproduction $[50,108,109,114]$, food intake [113], and in the drug/ immune response $[52,107,111,112]$.

\section{Availability of supporting data}

The complete transcriptomics dataset including all relevant parameters has been deposited to the National Center for Biotechnology Information (NCBI) database repository 'Gene Expression Omnibus' (GEO accession number: GSE63162) [115].

\section{Additional files}

\begin{abstract}
Additional file 1: Table S1. New names (column 1; uploaded at iBeetle genome browser [48]) based on chromosomal localization and corresponding previous names from Richards et al. 2008 [7] (column 2; uploaded at Beetle Base) as well as Foret and Maleska [16,63] respectively from Vieira and Rozas [12] (column 3). The AA sequence including the signal peptide with the tryptic fragments identified by MALDI-TOF MS highlighted in red (column 4). The length of the pre-peptide (column 5) and mature peptide (column 6) in AA. The predicted length of the signal peptide (column 7; based on SignalP4.1 [50]). The molecular mass of the pre-peptide (column 8) and the mature peptide (column 9). The superfamily identified by the conserved domain database [51] (column 10), as well the probability as e-value (column 11). The confidence of the signal peptide [50] (column 12). The number of cysteines in the mature peptide (column 13). The Cysteine formula adjusted to the six highly conserved cysteines (column 14; including C-OBPs). The isoelectric point (based on endmemo [116], column 15). The position of alpha helices (based on jpred [117], column 16). Whether it was confirmed by cloning from CDNA (column 17). Primer sequences used for cloning from cDNA (columns 18 and 19). The reads per kilobase of exon model per million mapped reads of the different tissue samples (columns 20-34) The calculated fold-changes over body, and the corresponding p-values (DESeq package [80], column 35-42).
\end{abstract}

Additional file 2: Figure S1. Midpoint-rooted phylogenetic tree of CSP (A) and OBP (B) sequences from Tribolium castaneum (green branches), Drosophila melanogaster (red branches), and Anopheles gambiae (blue branches). Outer rings represent the expression in body, 'mouthparts' (Tribolium: palps, mandible, labrum and labium; Drosophila:palp and proboscis; Anopheles: maxillary palp) and antenna as percentage compared to the highest expressed gene according to the scale in the right middle. Please note that the methods used to obtain the different expression data (RNAseq and microarray) are not directly comparable. Thus, this figure can only give an impression of the tissue-specific abundance of the transcripts. The scale bars within the trees represent 1 amino acid substitution per site. Inner ring in B indicates the phylogenetic subclass (classic in black, ABPII in grey, C-OBP in green, C+ OBP in blue, D7 in yellow, Dimer in red). Numbers on branches show values of 100 times replication bootstrap analysis higher than 70

Additional file 3: Table S2. Proteins of antennal extracts identified by MALDI-TOF-TOF MS-fingerprinting: accession number, molecular weight protein isoelectric point, peptide count, ion score, and Pfam prediction.

Additional file 4: Figure S2. Comparison of expression level of CSPS and OBPs in male and female heads (missing antennae but including mouthparts). Scatter plot of the RPKM values of the CSPs (in pink) and OBPs (classic in black, ABPIl in grey, C-OBP in green, C+ OBP in blue). The dotted lines represent a fivefold difference.

Additional file 5: Figure S3. Comparison of expression level of CSPS and OBPs in male and female mouthparts. Scatter plot of the RPKM values of the CSPS (in pink) and OBPs (classic in black, ABPII in grey, $\mathrm{C}-\mathrm{OBP}$ in green, $\mathrm{C}+\mathrm{OBP}$ in blue). The dotted lines represent a fivefold difference.

Additional file 6: Figure S4. Comparison of expression level of CSPS and OBPs in male and female legs. Scatter plot of the RPKM values of the CSPs (in pink) and OBPs (classic in black, ABPII in grey, C-OBP in green, $\mathrm{C}+\mathrm{OBP}$ in blue). The dotted lines represent a fivefold difference.

Additional file 7: Figure S5. Comparison of expression level of CSPS and OBPs in male and female bodies. Scatter plot of the RPKM values of the CSPs (in pink) and OBPs (classic in black, ABPII in grey, C-OBP in green, $C+O B P$ in blue). The dotted lines represent a five fold difference.

Competing interests

The authors declare that they have no competing interests.

\section{Authors' contributions}

EAW, SS, JS, SA, and SD conceived and initiated the project. SD, SA, and JK performed the experiments. SD, GO, and LO analyzed the data and performed the bioinformatics. LG and MS provided the gene sets and 
browser presentation. SD created the figures and wrote the first draft of the manuscript. SD and EAW revised the manuscript. All authors critically read, commented on, and approved of the manuscript.

\section{Acknowledgements}

We thank the transcriptome analysis laboratory (TAL, University Medical Center Göttingen, Germany), especially Gabriela Salinas-Riester for sequencing and technical support, Lotte Sogaard-Andersen from the Max Planck Institute for Terrestrial Microbiology (Marburg, Germany) for providing the MALDI TOF, and Montserrat Torres Oliva for technical and bioinformatics advice. This work was partially funded by the DFG Priority Programme "Integrative Analysis of Olfaction" (SPP 1392, SHA 678/13-1, SCHU 1135/13-1, and WI 1797/4-1).

\section{Author details}

'Department of Developmental Biology, Georg-August-University Goettingen, Johann-Friedrich-Blumenbach-Institute for Zoology and Anthropology, GZMB, Ernst-Caspari-Haus, Justus-von-Liebig-Weg 11, Goettingen 37077 , Germany. ${ }^{2}$ Department of Forest Zoology and Forest Conservation, Georg-August-University Goettingen, Buesgen-Institute, Buesgenweg 3, Goettingen 37077, Germany. ${ }^{3} \mathrm{MPI}$ for Terrestrial Microbiology, Karl-von-Frisch-Straße 10, Marburg D-35043, Germany. ${ }^{4}$ University of Greifswald, Institute for Mathematics and Computer Science, Walther-Rathenau-Straße 47, Greifswald D-17487, Germany. ${ }^{5}$ Functional Genomics Center Zurich, Winterthurerstr. 190, Zurich 8057, Switzerland ${ }^{6}$ Department of Biology - Animal Physiology, Philipps-University Marburg Karl-von-Frisch-Str. 8, Marburg 35032, Germany. 'Present address: Faculty of Science and Technology, Free University of Bolzano, Piazza Università 5, Bolzano 39100, Italy.

\section{Received: 5 August 2014 Accepted: 9 December 2014}

Published: 18 December 2014

\section{References}

1. Sokoloff A: The Genetics of Tribolium and Related Species, Volume 1. New York: Academic Press; 1966.

2. Bucher G, Scholten J, Klingler M: Parental RNAi in Tribolium (Coleoptera). Curr Biol CB 2002, 12:R85-R86.

3. Tomoyasu Y, Denell RE: Larval RNAi in Tribolium (Coleoptera) for analyzing adult development. Dev Genes Evol 2004, 214:575-578.

4. Trauner J, Schinko J, Lorenzen MD, Shippy TD, Wimmer EA, Beeman RW, Klingler M, Bucher G, Brown SJ: Large-scale insertional mutagenesis of a coleopteran stored grain pest, the red flour beetle Tribolium castaneum, identifies embryonic lethal mutations and enhancer traps. BMC Biol 2009, 7:73.

5. Schinko JB, Weber M, Viktorinova I, Kiupakis A, Averof M, Klingler M, Wimmer EA, Bucher G: Functionality of the GAL4/UAS system in Tribolium requires the use of endogenous core promoters. BMC Dev Biol 2010, 10:53.

6. Schinko JB, Hillebrand K, Bucher G: Heat shock-mediated misexpression of genes in the beetle Tribolium castaneum. Dev Genes Evol 2012, 222:287-298.

7. Richards S, Gibbs RA, Weinstock GM, Brown SJ, Denell R, Beeman RW, Gibbs R, Beeman RW, Brown SJ, Bucher G, Friedrich M, Grimmelikhuijzen CJP, Klingler M, Lorenzen M, Richards S, Roth S, Schröder R, Tautz D, Zdobnov EM, Muzny D, Gibbs RA, Weinstock GM, Attaway T, Bell S, Buhay CJ, Chandrabose MN, Chavez D, Clerk-Blankenburg KP, Cree A, Dao M, et al: The genome of the model beetle and pest Tribolium castaneum. Nature 2008, 452:949-955.

8. Kim HS, Murphy T, Xia J, Caragea D, Park Y, Beeman RW, Lorenzen MD, Butcher S, Manak JR, Brown SJ: BeetleBase in 2010: revisions to provide comprehensive genomic information for Tribolium castaneum. Nucleic Acids Res 2010, 38(Database issue):D437-D442.

9. de Santis F, François M-C, Merlin C, Pelletier J, Maïbèche-Coisné M, Conti E, Jacquin-Joly E: Molecular Cloning and in Situ Expression Patterns of Two New Pheromone-Binding Proteins from the Corn Stemborer Sesamia nonagrioides. J Chem Ecol 2006, 32:1703-1717.

10. Angeli S, Ceron F, Scaloni A, Monti M, Monteforti G, Minnocci A, Petacchi R, Pelosi P: Purification, structural characterization, cloning and immunocytochemical localization of chemoreception proteins from Schistocerca gregaria. Eur J Biochem 1999, 262:745-754.
11. Pelosi P, Zhou J-J, Ban LP, Calvello M: Soluble proteins in insect chemical communication. Cell Mol Life Sci CMLS 2006, 63:1658-1676.

12. Wanner KW, Willis LG, Theilmann DA, Isman MB, Feng Q, Plettner E: Analysis of the Insect OS-D-Like Gene Family. J Chem Ecol 2004, 30:889-911.

13. Liu R, He X, Lehane S, Lehane M, Hertz-Fowler C, Berriman M, Field LM, Zhou J-J: Expression of chemosensory proteins in the tsetse fly Glossina morsitans morsitans is related to female host-seeking behaviour. Insect Mol Biol 2012, 21:41-48.

14. Leal WS: Odorant Reception in Insects: Roles of Receptors, Binding Proteins, and Degrading Enzymes. Annu Rev Entomol 2013, 58:373-391.

15. Steinbrecht RA: Odorant-binding proteins: expression and function. Ann N Y Acad Sci 1998, 855:323-332.

16. Vogt $R G$, Riddiford LM: Pheromone binding and inactivation by moth antennae. Nature 1981, 293:161-163.

17. Vieira FG, Rozas J: Comparative Genomics of the Odorant-Binding and Chemosensory Protein Gene Families across the Arthropoda: Origin and Evolutionary History of the Chemosensory System. Genome Biol Evol 2011, 3:476-490.

18. Sandler BH, Nikonova L, Leal WS, Clardy J: Sexual attraction in the silkworm moth: structure of the pheromone-binding-protein-bombykol complex. Chem Biol 2000, 7:143-151.

19. Briand L, Swasdipan N, Nespoulous C, Bézirard V, Blon F, Huet J-C, Ebert P, Penollet J-C: Characterization of a chemosensory protein (ASP3C) from honeybee (Apis mellifera L.) as a brood pheromone carrier. Eur J Biochem FEBS 2002, 269:4586-4596.

20. Scaloni A, Monti M, Angeli S, Pelosi P: Structural analysis and disulfide-bridge pairing of two odorant-binding proteins from Bombyx mori. Biochem Biophys Res Commun 1999, 266:386-391.

21. Foret $S$, Maleszka R: Function and evolution of a gene family encoding odorant binding-like proteins in a social insect, the honey bee (Apis mellifera). Genome Res 2006, 16:1404-1413.

22. Hekmat-Scafe DS, Scafe CR, McKinney AJ, Tanouye MA: Genome-wide analysis of the odorant-binding protein gene family in Drosophila melanogaster. Genome Res 2002, 12:1357-1369.

23. Spinelli S, Lagarde A, lovinella I, Legrand P, Tegoni M, Pelosi P, Cambillau C: Crystal structure of Apis mellifera OBP14, a C-minus odorant-binding protein, and its complexes with odorant molecules. Insect Biochem Mol Biol 2012, 42:41-50.

24. Gu S-H, Wang S-Y, Zhang X-Y, Ji P, Liu J-T, Wang G-R, Wu K-M, Guo Y-Y, Zhou J-J, Zhang Y-J: Functional Characterizations of Chemosensory Proteins of the Alfalfa Plant Bug Adelphocoris lineolatus Indicate Their Involvement in Host Recognition. PLoS One 2012, 7:e42871.

25. Ozaki M: Ant Nestmate and Non-Nestmate Discrimination by a Chemosensory Sensillum. Science 2005, 309:311-314.

26. Jacquin-Joly E, Vogt RG, François M-C, Meillour PN-L: Functional and Expression Pattern Analysis of Chemosensory Proteins Expressed in Antennae and Pheromonal Gland of Mamestra brassicae. Chem Senses 2001, 26:833-844.

27. Andersson MN, Grosse-Wilde E, Keeling Cl, Bengtsson JM, Yuen MM, Li M, Hillbur Y, Bohlmann J, Hansson BS, Schlyter F: Antennal transcriptome analysis of the chemosensory gene families in the tree killing bark beetles, Ips typographus and Dendroctonus ponderosae (Coleoptera: Curculionidae: Scolytinae). BMC Genomics 2013, 14:198.

28. González D, Zhao Q, McMahan C, Velasquez D, Haskins WE, Sponsel V, Cassill A, Renthal R: The major antennal chemosensory protein of red imported fire ant workers. Insect Mol Biol 2009, 18:395-404.

29. Liu X, Luo Q, Zhong G, Rizwan-Ul-Haq M, Hu M: Molecular characterization and expression pattern of four chemosensory proteins from diamondback moth, Plutella xylostella (Lepidoptera: Plutellidae). J Biochem (Tokyo) 2010, 148:189-200.

30. Forstner $\mathrm{M}$, Breer $\mathrm{H}$, Krieger J: A receptor and binding protein interplay in the detection of a distinct pheromone component in the silkmoth Antheraea polyphemus. Int J Biol Sci 2009, 5:745-757.

31. Grosse-Wilde E, Gohl T, Bouché E, Breer H, Krieger J: Candidate pheromone receptors provide the basis for the response of distinct antennal neurons to pheromonal compounds. Eur J Neurosci 2007, 25:2364-2373.

32. Grosse-Wilde E, Svatos A, Krieger J: A pheromone-binding protein mediates the bombykol-induced activation of a pheromone receptor in vitro. Chem Senses 2006, 31:547-555.

33. Hallem EA, Ho MG, Carlson JR: The molecular basis of odor coding in the Drosophila antenna. Cell 2004, 117:965-979. 
34. Syed Z, Ishida Y, Taylor K, Kimbrell DA, Leal WS: Pheromone reception in fruit flies expressing a moth's odorant receptor. Proc Natl Acad Sci U S A 2006, 103:16538-16543

35. Xu P, Atkinson R, Jones DNM, Smith DP: Drosophila OBP LUSH is required for activity of pheromone-sensitive neurons. Neuron 2005, 45:193-200.

36. Arya GH, Weber AL, Wang P, Magwire MM, Negron YLS, Mackay TFC Anholt RRH: Natural Variation, Functional Pleiotropy and Transcriptional Contexts of Odorant Binding Protein Genes in Drosophila melanogaster. Genetics 2010, 186:1475-1485.

37. Krieger MJB, Ross KG: Molecular evolutionary analyses of the odorantbinding protein gene Gp-9 in fire ants and other Solenopsis species. Mol Biol Evol 2005, 22:2090-2103.

38. Swarup S, Williams TI, Anholt RRH: Functional dissection of Odorant binding protein genes in Drosophila melanogaster. Genes Brain Behav 2011, 10:648-657.

39. Biessmann H, Andronopoulou E, Biessmann MR, Douris V, Dimitratos SD, Eliopoulos E, Guerin PM, latrou K, Justice RW, Kröber T, Marinotti O, Tsitoura P, Woods DF, Walter MF: The Anopheles gambiae Odorant Binding Protein 1 (AgamOBP1) Mediates Indole Recognition in the Antennae of Female Mosquitoes. PLoS One 2010, 5:e9471.

40. Pelletier J, Guidolin A, Syed Z, Cornel AJ, Leal WS: Knockdown of a Mosquito Odorant-binding Protein Involved in the Sensitive Detection of Oviposition Attractants. J Chem Ecol 2010, 36:245-248.

41. Gomez-Diaz C, Reina JH, Cambillau C, Benton R: Ligands for PheromoneSensing Neurons Are Not Conformationally Activated Odorant Binding Proteins. PLoS Biol 2013, 11:e1001546.

42. Pitts RJ, Rinker DC, Jones PL, Rokas A, Zwiebel $\amalg$ : Transcriptome profiling of chemosensory appendages in the malaria vector Anopheles gambiae reveals tissue- and sex-specific signatures of odor coding. BMC Genomics 2011, 12:271.

43. Farhadian SF, Suárez-Fariñas M, Cho CE, Pellegrino M, Vosshall LB: Postfasting olfactory, transcriptional, and feeding responses in Drosophila. Physiol Behav 2012, 105:544-553.

44. Pelletier J, Leal WS: Genome Analysis and Expression Patterns of OdorantBinding Proteins from the Southern House Mosquito Culex pipiens quinquefasciatus. PLoS One 2009, 4:e6237.

45. Zhang Y-N, Jin J-Y, Jin R, Xia Y-H, Zhou J-J, Deng J-Y, Dong S-L: Differential Expression Patterns in Chemosensory and Non-Chemosensory Tissues of Putative Chemosensory Genes Identified by Transcriptome Analysis of Insect Pest the Purple Stem Borer Sesamia inferens (Walker). PLoS One 2013, 8:e69715.

46. Zheng W, Peng W, Zhu C, Zhang Q, Saccone G, Zhang H: Identification and Expression Profile Analysis of Odorant Binding Proteins in the Oriental Fruit Fly Bactrocera dorsalis. Int J Mol Sci 2013, 14:14936-14949.

47. Gong D-P, Zhang H, Zhao P, Lin Y, Xia Q-Y, Xiang Z-H: Identification and expression pattern of the chemosensory protein gene family in the silkworm, Bombyx mori. Insect Biochem Mol Biol 2007, 37:266-277.

48. Zhou J-J, Kan Y, Antoniw J, Pickett JA, Field LM: Genome and EST Analyses and Expression of a Gene Family with Putative Functions in Insect Chemoreception. Chem Senses 2006, 31:453-465.

49. Dani FR, Michelucci E, Francese S, Mastrobuoni G, Cappellozza S, Marca GL, Niccolini A, Felicioli A, Moneti G, Pelosi P: Odorant-Binding Proteins and Chemosensory Proteins in Pheromone Detection and Release in the Silkmoth Bombyx mori. Chem Senses 2011, 36:335-344.

50. Sun Y-L, Huang L-Q, Pelosi P, Wang C-Z: Expression in Antennae and Reproductive Organs Suggests a Dual Role of an Odorant-Binding Protein in Two Sibling Helicoverpa Species. PLoS One 2012, 7:e30040.

51. Maleszka J, Forêt S, Saint R, Maleszka R: RNAi-induced phenotypes suggest a novel role for a chemosensory protein CSP5 in the development of embryonic integument in the honeybee (Apis mellifera). Dev Genes Evol 2007, 217:189-196.

52. Levy F, Bulet $P$, Ehret-Sabatier L: Proteomic Analysis of the Systemic Immune Response of Drosophila. Mol Cell Proteomics 2004, 3:156-166.

53. Nomura A, Kawasaki K, Kubo T, Natori S: Purification and localization of p10, a novel protein that increases in nymphal regenerating legs of Periplaneta americana (American cockroach). Int J Dev Biol 1992, 36:391-398.

54. Schütz S, Weißbecker B, Schroth P, Schöning MJ: Linkage of Inanimate Structures to Biological Systems - Smart Materials in Biological Microand Nanosystems. In Smart Mater. Edited by Hoffmann K-H. Heidelberg: Springer Berlin; 2001:149-157.
55. Di Pietrantonio F, Cannatà D, Benetti M, Verona E, Varriale A, Staiano M, D'Auria S: Detection of odorant molecules via surface acoustic wave biosensor array based on odorant-binding proteins. Biosens Bioelectron 2013, 41:328-334.

56. Schütz S, Weißbecker B, Koch UT, Hummel HE: Detection of volatiles released by diseased potato tubers using a biosensor on the basis of intact insect antennae. Biosens Bioelectron 1999, 14:221-228.

57. Johne $A B$, Weissbecker $B$, Schütz $S$ : Approaching risk assessment of complex disease development in horse chestnut trees: a chemical ecologist's perspective. J Appl Entomol 2008, 132:349-359.

58. Zhou J-J, Field LM, He XL: Insect Odorant-Binding Proteins: Do They Offer an Alternative Pest Control Strategy? Outlooks Pest Manag 2010, 21:31-34.

59. Pannure A, Mutthuraju GP, Imran S: The sense of smell in insects: a target for pest management? - A review. Curr Biot 2012, 6:399-419.

60. Abdel-Sattar E, Zaitoun AA, Farag MA, Gayed SHE, Harraz FMH: Chemical composition, insecticidal and insect repellent activity of Schinus molle L. leaf and fruit essential oils against Trogoderma granarium and Tribolium castaneum. Nat Prod Res 2010, 24:226-235.

61. Andreev D, Kreitman M, Phillips TW, Beeman RW, ffrench-Constant RH: Multiple origins of cyclodiene insecticide resistance in Tribolium castaneum (Coleoptera: Tenebrionidae). J Mol Evol 1999, 48:615-624.

62. da Affonso RS, Affonso RS, Guimarães AP, Oliveira AA, Slana GBC, França TCC: Applications of molecular modeling in the design of new insect repellents targeting the odorant binding protein of Anopheles gambiae. J Braz Chem Soc 2013, 24:473-482.

63. Ridley AW, Hereward JP, Daglish GJ, Raghu S, Collins PJ, Walter GH: The spatiotemporal dynamics of Tribolium castaneum (Herbst): adult flight and gene flow. Mol Ecol 2011, 20:1635-1646.

64. Langmead B, Salzberg SL: Fast gapped-read alignment with Bowtie 2. Nat Methods 2012, 9:357-359.

65. Forêt S, Wanner KW, Maleszka R: Chemosensory proteins in the honey bee: Insights from the annotated genome, comparative analyses and expressional profiling. Insect Biochem Mol Biol 2007, 37:19-28.

66. Altschul SF, Madden TL, Schäffer AA, Zhang J, Zhang Z, Miller W, Lipman DJ: Gapped BLAST and PSI-BLAST: a new generation of protein database search programs. Nucleic Acids Res 1997, 25:3389-3402.

67. Stajich JE, Block D, Boulez K, Brenner SE, Chervitz SA, Dagdigian C, Fuellen G, Gilbert JGR, Korf I, Lapp H, Lehväslaiho H, Matsalla C, Mungall CJ, Osborne BI, Pocock MR, Schattner P, Senger M, Stein LD, Stupka E, Wilkinson MD, Birney E: The Bioperl toolkit: Perl modules for the life sciences. Genome Res 2002, 12:1611-1618.

68. Li H, Handsaker B, Wysoker A, Fennell T, Ruan J, Homer N, Marth G, Abecasis G, Durbin R: The Sequence Alignment/Map format and SAMtools. Bioinformatics 2009, 25:2078-2079.

69. Patel RK, Jain M: NGS QC Toolkit: A Toolkit for Quality Control of Next Generation Sequencing Data. PLoS One 2012, 7:e30619.

70. Grabherr MG, Haas BJ, Yassour M, Levin JZ, Thompson DA, Amit I, Adiconis X, Fan L, Raychowdhury R, Zeng Q, Chen Z, Mauceli E, Hacohen N, Gnirke A, Rhind N, di Palma F, Birren BW, Nusbaum C, Lindblad-Toh K, Friedman N, Regev A: Full-length transcriptome assembly from RNA-Seq data without a reference genome. Nat Biotechnol 2011, 29:644-652.

71. Stanke M, Diekhans M, Baertsch R, Haussler D: Using native and syntenically mapped CDNA alignments to improve de novo gene finding. Bioinformatics 2008, 24:637-644.

72. iBeetle consortium: Http://ibeetle.uni-Goettingen.de.

73. iBeetle genome browser, Stanke M, Bucher G, Klingler M: Http://bioinf.uniGreifswald.de/tcas/.

74. Kent WJ: BLAT-The BLAST-Like Alignment Tool. Genome Res 2002, 12:656-664.

75. Petersen TN, Brunak S, von Heijne G, Nielsen H: SignalP 4.0: discriminating signal peptides from transmembrane regions. Nat Methods 2011, 8:785-786.

76. Marchler-Bauer A, Lu S, Anderson JB, Chitsaz F, Derbyshire MK, DeWeeseScott C, Fong JH, Geer LY, Geer RC, Gonzales NR, Gwadz M, Hurwitz DI, Jackson JD, Ke Z, Lanczycki CJ, Lu F, Marchler GH, Mullokandov M, Omelchenko MV, Robertson CL, Song JS, Thanki N, Yamashita RA, Zhang D, Zhang N, Zheng C, Bryant SH: CDD: a Conserved Domain Database for the functional annotation of proteins. Nucleic Acids Res 2011, 39(Database issue):D225-D229.

77. Pavlidis P, Noble WS: Matrix2png: a utility for visualizing matrix data. Bioinformatics 2003, 19:295-296. 
78. Inkscape: http:/Www.inkscape.org.

79. R: A Language and Environment for Statistical Computing. [http://www.Rproject.org].

80. Anders S, Huber W: Differential expression analysis for sequence count data. Genome Biol 2010, 11:R106.

81. Gentleman RC, Carey VJ, Bates DM, Bolstad B, Dettling M, Dudoit S, Ellis B, Gautier L, Ge Y, Gentry J, Hornik K, Hothorn T, Huber W, lacus S, Irizarry R, Leisch F, Li C, Maechler M, Rossini AJ, Sawitzki G, Smith C, Smyth G, Tierney L, Yang JY, Zhang J: Bioconductor: open software development for computational biology and bioinformatics. Genome Biol 2004, 5:R80

82. Katoh K, Kuma K, Toh H, Miyata T: MAFFT version 5: improvement in accuracy of multiple sequence alignment. Nucleic Acids Res 2005, 33:511-518.

83. Stamatakis A: RAxML-VI-HPC: maximum likelihood-based phylogenetic analyses with thousands of taxa and mixed models. Bioinforma Oxf Eng 2006, 22:2688-2690.

84. Kapushesky M, Adamusiak T, Burdett T, Culhane A, Farne A, Filippov A, Holloway E, Klebanov A, Kryvych N, Kurbatova N, Kurnosov P, Malone J, Melnichuk O, Petryszak R, Pultsin N, Rustici G, Tikhonov A, Travillian RS, Williams E, Zorin A, Parkinson H, Brazma A: Gene Expression Atlas updatea value-added database of microarray and sequencing-based functional genomics experiments. Nucleic Acids Res 2011, 40:D1077-D1081.

85. Letunic I, Bork P: Interactive Tree Of Life (iTOL): an online tool for phylogenetic tree display and annotation. Bioinformatics 2007, 23:127-128.

86. Wogulis M, Morgan T, Ishida Y, Leal WS, Wilson DK: The crystal structure of an odorant binding protein from Anopheles gambiae: Evidence for a common ligand release mechanism. Biochem Biophys Res Commun 2006 339:157-164.

87. Zhou J-J, Robertson G, He X, Dufour S, Hooper AM, Pickett JA, Keep NH Field LM: Characterisation of Bombyx mori Odorant-binding Proteins Reveals that a General Odorant-binding Protein Discriminates Between Sex Pheromone Components. J Mol Biol 2009, 389:529-545.

88. Vieira FG, Forêt S, He X, Rozas J, Field LM, Zhou J-J: Unique Features of Odorant-Binding Proteins of the Parasitoid Wasp Nasonia vitripennis Revealed by Genome Annotation and Comparative Analyses. PLoS One 2012, 7:e43034.

89. Zhou J-J: Chapter Ten - Odorant-Binding Proteins in Insects. In Vitam Horm. Volume Volume 83. Edited by Litwack G. Amsterdam: Academic Press; 2010:241-272 [Pheromones].

90. Eirín-López JM, Rebordinos L, Rooney AP, Rozas J: The Birth-and-Death Evolution of Multigene Families Revisited. In Genome Dyn. Volume 7. Edited by Garrido-Ramos MA. Basel: S. KARGER AG; 2012:170-196.

91. Zhou J-J, Huang W, Zhang G-A, Pickett JA, Field LM: "Plus-C" odorantbinding protein genes in two Drosophila species and the malaria mosquito Anopheles gambiae. Gene 2004, 327:117-129.

92. Dreyer D, Vitt H, Dippel S, Goetz B, El Jundi B, Kollmann M, Huetteroth W, Schachtner J: 3D Standard Brain of the Red Flour Beetle Tribolium Castaneum: A Tool to Study Metamorphic Development and Adult Plasticity. Front Syst Neurosci 2010, 4:3.

93. Alabi T, Marion-Poll F, Danho M, Mazzucchelli GD, De Pauw E, Haubruge E, Francis $\mathrm{F}$ : Identification of taste receptors and proteomic characterization of the antenna and legs of Tribolium brevicornis, a stored food product pest. Insect Mol Biol 2013, 23:1-12.

94. Engsontia P, Sanderson AP, Cobb M, Walden KKO, Robertson HM, Brown S: The red flour beetle's large nose: an expanded odorant receptor gene family in Tribolium castaneum. Insect Biochem Mol Biol 2008, 38:387-397.

95. Li J, Lehmann S, Weißbecker B, Ojeda Naharros I, Schütz S, Joop G, Wimmer EA: Odoriferous Defensive Stink Gland Transcriptome to Identify Novel Genes Necessary for Quinone Synthesis in the Red Flour Beetle, Tribolium castaneum. PLoS Genet 2013, 9:e1003596.

96. Xu PX, Zwiebel LJ, Smith DP: Identification of a distinct family of genes encoding atypical odorant-binding proteins in the malaria vector mosquito, Anopheles gambiae. Insect Mol Biol 2003, 12:549-560.

97. Gong D-P, Zhang H-J, Zhao P, Xia Q-Y, Xiang Z-H: The Odorant Binding Protein Gene Family from the Genome of Silkworm, Bombyx mori. BMC Genomics 2009, 10:332.

98. Vieira FG, Sánchez-Gracia A, Rozas J: Comparative genomic analysis of the odorant-binding protein family in 12 Drosophila genomes: purifying selection and birth-and-death evolution. Genome Biol 2007, 8:R235.
99. Librado $P$, Rozas J: Uncovering the functional constraints underlying the genomic organisation of the Odorant-Binding Protein genes. Genome Biol Evol 2013, 5:2096-2108.

100. Jafari S, Alkhori L, Schleiffer A, Brochtrup A, Hummel T, Alenius M: Combinatorial Activation and Repression by Seven Transcription Factors Specify Drosophila Odorant Receptor Expression. PLOS Biol 2012, 10:e1001280

101. Leal WS, Nikonova L, Peng G: Disulfide structure of the pheromone binding protein from the silkworm moth, Bombyx mori. FEBS Lett 1999, 464:85-90.

102. Hungate EA, Earley EJ, Boussy IA, Turissini DA, Ting C-T, Moran JR, Wu M-L, Wu C-I, Jones CD: A Locus in Drosophila sechellia Affecting Tolerance of a Host Plant Toxin. Genetics 2013, 195:1063-1075.

103. Qiao H-L, Deng P-Y, Li D-D, Chen M, Jiao Z-J, Liu Z-C, Zhang Y-Z, Kan Y-C: Expression analysis and binding experiments of chemosensory proteins indicate multiple roles in Bombyx mori. J Insect Physiol 2013, 59:667-675.

104. Ju Q, Li X, Jiang X-J, Qu M-J, Guo X-Q, Han Z-J, Li F: Transcriptome and Tissue-Specific Expression Analysis of Obp and Csp Genes in the Dark Black Chafer. Arch Insect Biochem Physiol 2014, 87:177-200.

105. Qiao H, He X, Schymura D, Ban L, Field L, Dani FR, Michelucci E, Caputo B, della Torre A, latrou K, Zhou J-J, Krieger J, Pelosi P: Cooperative interactions between odorant-binding proteins of Anopheles gambiae. Cell Mol Life Sci 2010, 68:1799-1813.

106. Schultze A, Pregitzer P, Walter MF, Woods DF, Marinotti O, Breer H, Krieger J: The Co-Expression Pattern of Odorant Binding Proteins and Olfactory Receptors Identify Distinct Trichoid Sensilla on the Antenna of the Malaria Mosquito Anopheles gambiae. PLoS One 2013, 8:e69412.

107. Contreras E, Rausell C, Real MD: Proteome Response of Tribolium castaneum Larvae to Bacillus thuringiensis Toxin Producing Strains. PLoS One 2013, 8:e55330.

108. Xu J, Baulding J, Palli SR: Proteomics of Tribolium castaneum seminal fluid proteins: Identification of an angiotensin-converting enzyme as a key player in regulation of reproduction. J Proteomics 2013, 78:83-93.

109. Sirot LK, Poulson RL, McKenna MC, Girnary H, Wolfner MF, Harrington LC: Identity and transfer of male reproductive gland proteins of the dengue vector mosquito, Aedes aegypti: potential tools for control of female feeding and reproduction. Insect Biochem Mol Biol 2008, 38:176-189.

110. Ban L, Napolitano E, Serra A, Zhou X, lovinella I, Pelosi P: Identification of pheromone-like compounds in male reproductive organs of the oriental locust Locusta migratoria. Biochem Biophys Res Commun 2013, 437:620-624.

111. Liu GX, Xuan N, Chu D, Xie HY, Fan ZX, Bi YP, Picimbon J-F, Qin YC, Zhong ST, Li YF, Gao ZL, Pan WL, Wang GY, Rajashekar B: Biotype expression and insecticide response of Bemisia tabaci chemosensory protein-1. Arch Insect Biochem Physiol 2014, 85:137-151.

112. Xuan N, Guo X, Xie H-Y, Lou Q-N, Lu X-B, Liu G-X, Picimbon J-F: Increased expression of CSP and CYP genes in adult silkworm females exposed to avermectins. Insect Sci 2014, : (doi: 10.1111/1744-7917.12116).

113. Liu $Y-L$, Guo $H$, Huang $L-Q$, Pelosi $P$, Wang C-Z: Unique function of a chemosensory protein in the proboscis of two Helicoverpa species. J Exp Biol 2014, 217(Pt 10):1821-1826.

114. Findlay GD, Yi X, MacCoss MJ, Swanson WJ: Proteomics Reveals Novel Drosophila Seminal Fluid Proteins Transferred at Mating. PLoS Biol 2008, 6:e178.

115. GEO accession. http//www.ncbi.nlm.nih.gov/geo/query/acc.cgi?acc=GSE63162.

116. endmemo: http://www.endmemo.com/bio/proie.php.

117. jpred: http://www.compbio.dundee.ac.uk/www-jpred/index.html.

\section{doi:10.1186/1471-2164-15-1141}

Cite this article as: Dippel et al.: Tissue-specific transcriptomics, chromosomal localization, and phylogeny of chemosensory and odorant binding proteins from the red flour beetle Tribolium castaneum reveal subgroup specificities for olfaction or more general functions. BMC Genomics 2014 15:1141. 


\section{Additional files}

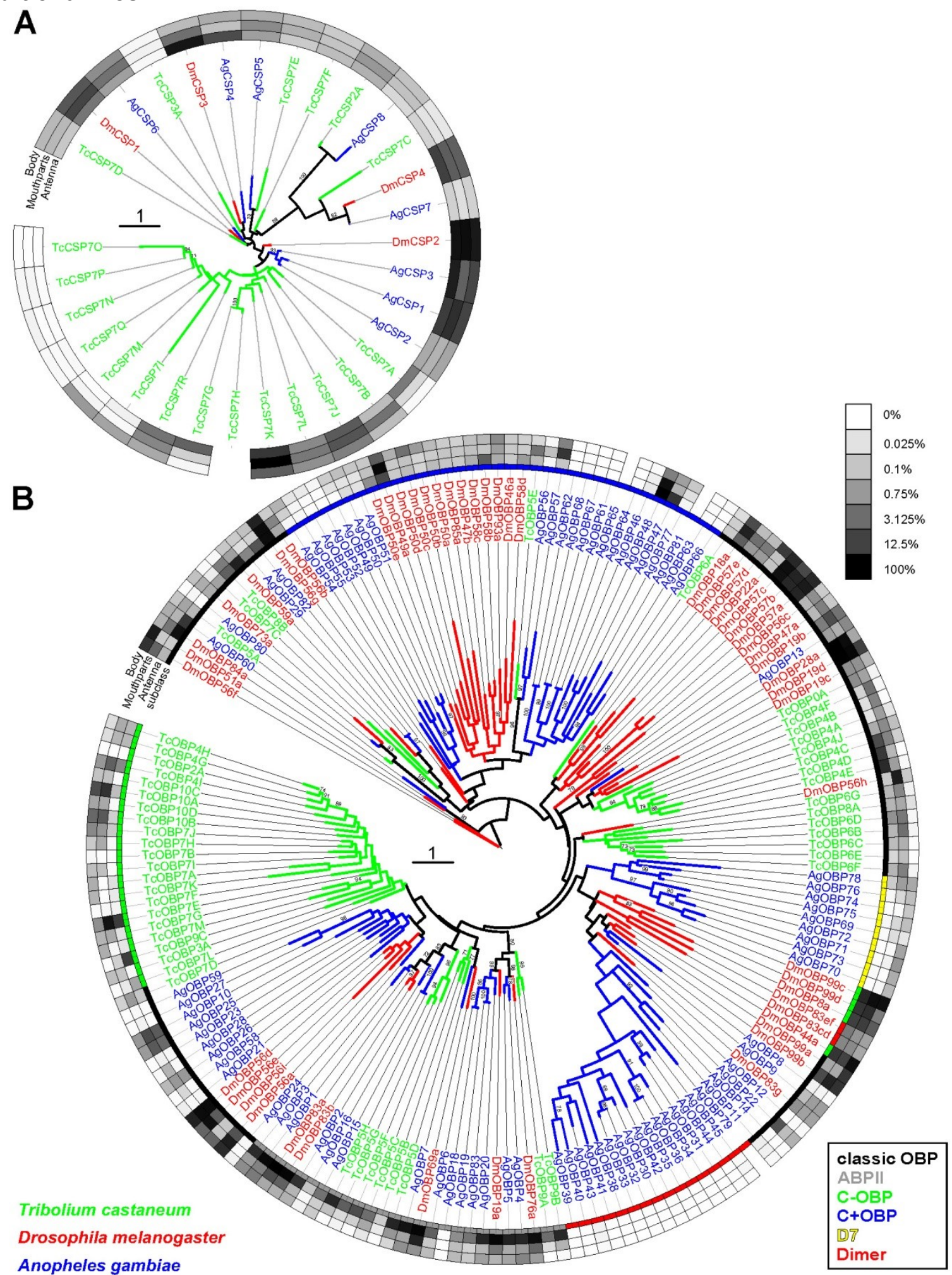

Additional file 2: Figure S1. 


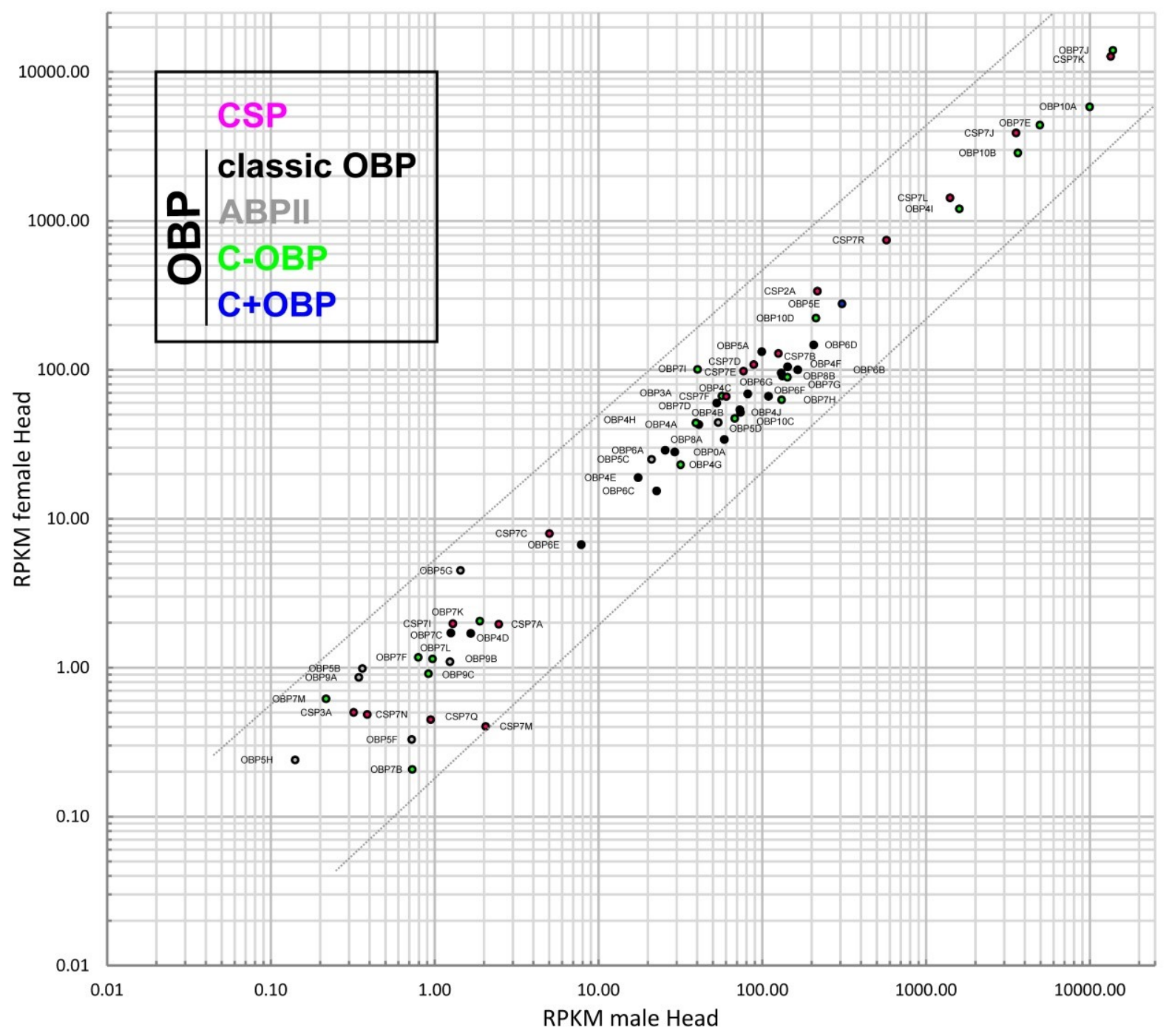

Additional file 4 . 


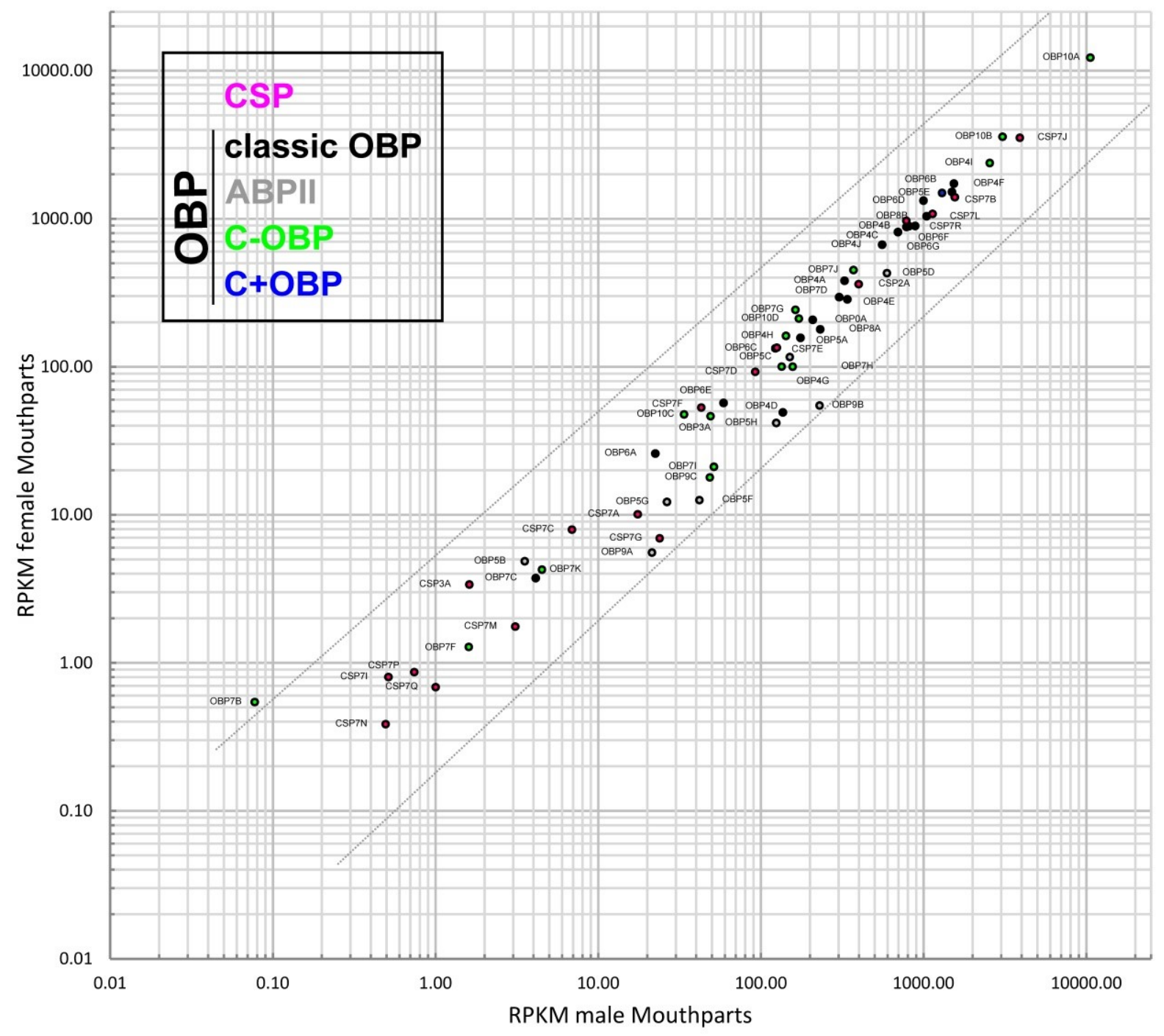

Additional file 5: Figure S3. 


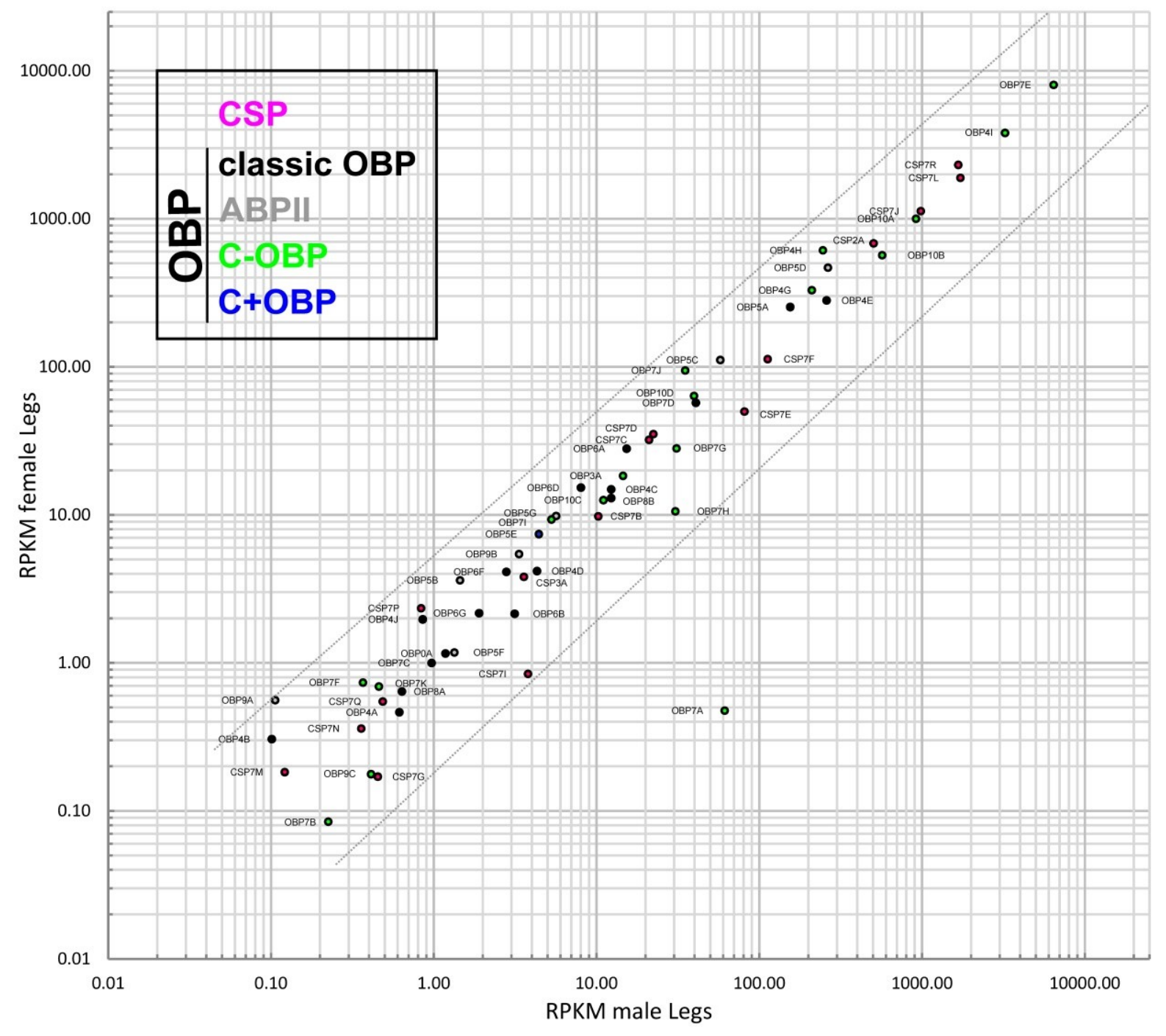

Additional file 6: Figure S4. 


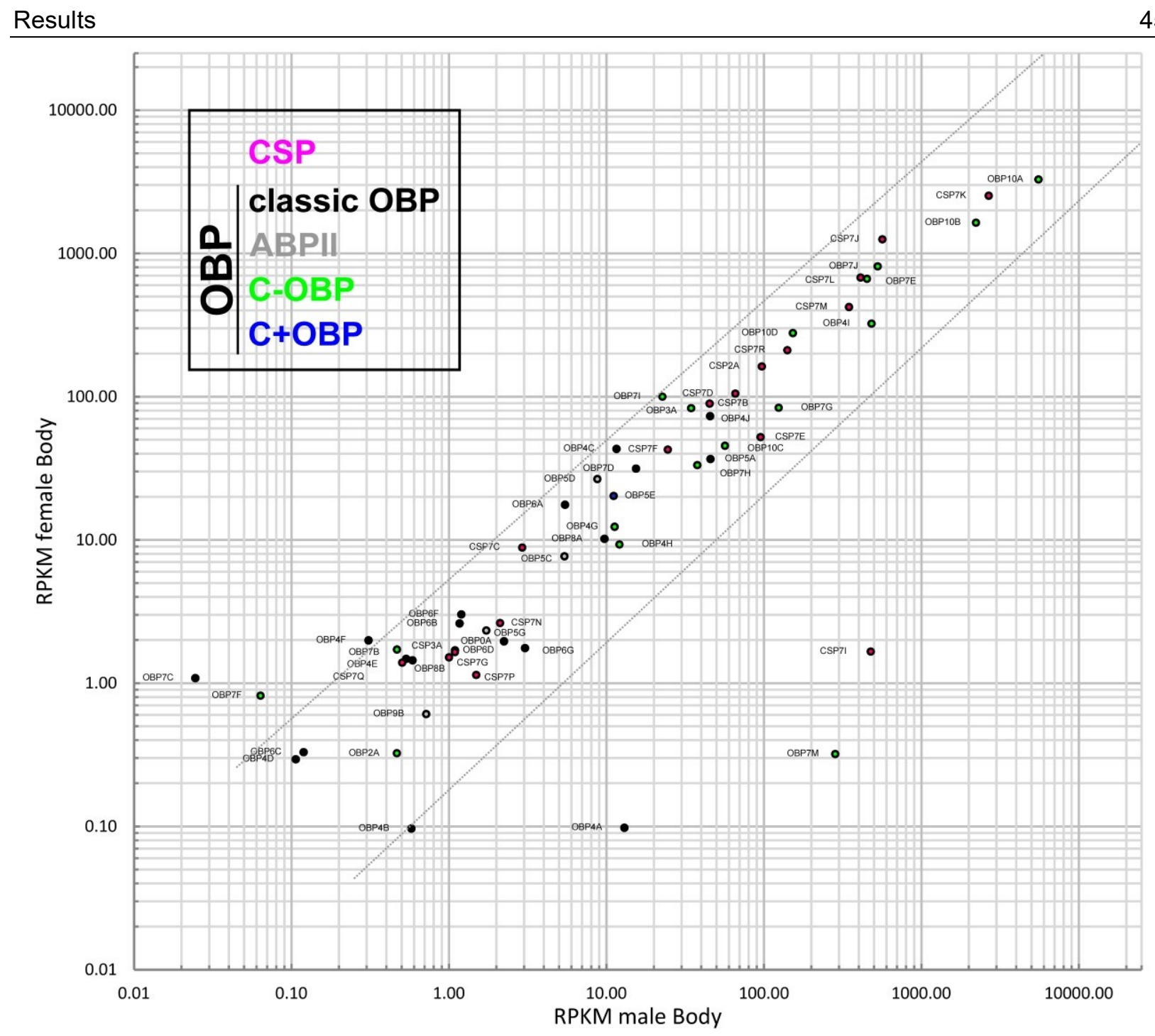

Additional file 7: Figure S5. 


\subsection{Morphological and Transcriptomic Analysis of a Beetle Chemosensory System Reveals a Gnathal Olfactory Center}

The focus of this section was the RNAseq based re-annotation of computational predicted gene models for IRs, GRs, ORs, SNMPs, and potential ODEs of T. castaneum, the chromsosomal localization and quantification of their expression, as well as the phylogenic comparison with related gene families of Drosophila melanogaster and Anopheles gambiae. Once complete, asolid evaluation of whether conserved genes show similar expression profile could be conducted. Furthermore, a detailed description of the anatomical structures involved in olfaction and gustation, such as the antennae, palps, and antennal lobe, should be performed through, immunohistochemistry, generation of transgenic reporter lines, backfills, and dye injections.

Authors: Stefan Dippel, Martin Kollmann, Georg Oberhofer, Alice Montino, Carolin Knoll, Milosz Krala, Karl-Heinz Rexer, Sergius Frank, Joachim Schachtner, Ernst A Wimmer

Status: Published in BMC Biology, 2016. 14:90 (doi: 10.1186/s12915-016-0304-z).

\section{My contributions:}

Drafting and revision of the manuscript.

Figure 1. Sensilla types and distribution on Tribolium castaneum antennae I.

A: Generation of the used transgenic line, data acquisition (confocal scanning), and visualization in AMIRA.

$\mathrm{C}-\mathrm{H}$ : Tissue collection, sectioning, most of the stainings and embeddings for the CLSM images, acquisition of some of the confocal scans.

Figure 2. Sensilla types and distribution on the antennae of $T$. castaneum II.

$\mathrm{H}$ and $\mathrm{I}$ : Tissue collection, sectioning, most of the staining and embedding

Figure 3. The central olfactory pathway of $T$. castaneum.

A-E: Assistance during backfilling of some of the samples.

$\mathrm{F}$ : Generation of the used transgenic line.

Figure 4. Orco- immunoreactive sensory neurons in the maxillary palp. Generation of the used transgenic line and tissue collection, sectioning, staining, and embedding

Figure 5. Antennal lobe tracts. About half of the tracer injections. 
Figure 6. Comparison of expression levels in male and female antenna. Tissue collection, RNA isolation, analysis (building a modified gene set by manual curation of the gene models; analysis of the readcounts), and complete visualization.

Figure 7. Expression of $T$. castaneum ionotropic glutamate-like receptors (IRs). Tissue collection, RNA isolation, analysis (building a modified gene set by manual curation of the gene models; analysis of the read-counts and statistical data), and complete visualization.

Figure 8. Phylogenetic tree of IRs. Tissue collection, RNA isolation, bioinformatic analysis (building a modified gene set by manual curation of the gene models; analysis of the read-counts and statistical data; and in silico collection of $D$. melanogaster and An. gambiae data, calculation of the relative expression levels and of parts of the phylogenetic tree), and visualization.

Figure 9. Expression of $T$. castaneum gustatory receptors (GRs). Same as figure 7.

Figure 10. Phylogenetic tree of gustatory receptors (GRs). Same as figure 8.

Figure 11. Expression of $T$. castaneums odorant receptors (ORs). Same as figure 7 and reinterpretation and integration of previously published data.

Figure 12. Phylogenetic tree of odorant receptors (ORs). Same as figure 8.

Figure 13. Expression of $T$. castaneums potential odorant degrading enzymes (ODEs). Same as figure 7 and identification of the candidates.

Figure 14. Expression of $T$. castaneums homologs of genes described as being involved in olfaction of $D$. melanogaster. Same as figure 13.

Figure 15. Expression of T. castaneums SNMPs. Same as figure 7.

Figure 16. Scheme of the T. castaneum head including the major components of the olfactory pathway. Providing some data for the illustration.

Additional file 1: Figure S1. Antibody staining against DsRed and Orco of the EF1-B-DsRed line. Preparation (tissue collection, sectioning, most of the staining and embedding), acquisition of some of the confocal scans.

Additional file 2: Figure S2. Orco localization in antennae and palp.

A: Cloning of TcasOrco.

B-C: Generation of the Orco knockdown beetles and same as additional file 1: Figure S1.

D: Same as additional file 1: Figure S1.

$\mathrm{E}$ : Generation of the used transgenic line.

F: Same as additional file 1: Figure S1.

Additional file 3: Figure S3. Comparison of sensilla type numbers on the antenna of Tribolium castaneum and chemosensory neurons entering the sensilla types. I: Basis for the analysis shown (tissue collection, sectioning, most of the staining and embedding for the CLSM images, scanning of some of the confocal stacks).

Additional file 6: Figure S4. Ipsilateral antennal projection. Same as figure 3 A-E.

Additional file 8: Table S1. Summary of the RNAseq data. Most of the analysis behind these data.

Additional file 9: Figure S5. IR gene tissue expression and chromosomal localization of IR and SNMP genes.

A: Same as Figure 7.

B: Data collection from previous publications, analysis (BLAST based genomic localization), and complete visualization.

Additional file 10: Figure S6. Phylogenetic tree of IRs. Same as figure 8.

Additional file 11: Figure S7. GR gene tissue expression and their chromosomal localization. Same as additional file 9: figure S5.

Additional file 12: Figure S8. Phylogenetic mid-point rooted tree of the GRs based on protein sequences. Same as figure 8.

Additional file 13: Figure S9. OR gene tissue expression and their chromosomal localization. Same as additional file 9: Figure S5.

Additional file 14: Figure S10. Phylogenetic tree of the ORs based on protein sequences. Same as figure 8.

Additional file 15: Figure S11. Chromosomal localization of potential T. castaneum ODE genes. Same as additional file 9: Figure S5.

Additional file 17: Figure S12. UAS responder lines in the absence of Gal4 driver. Generation of the used transgenic lines, except for UAS-tGFP, crossing, preparation and confocal scanning. 


\title{
Morphological and Transcriptomic Analysis of a Beetle Chemosensory System Reveals a Gnathal Olfactory Center
}

\author{
Stefan Dippel ${ }^{1 \dagger}$, Martin Kollmann ${ }^{2 \dagger}$, Georg Oberhofer ${ }^{3}$, Alice Montino', Carolin Knoll², Milosz Krala²,
} Karl-Heinz Rexer ${ }^{4}$, Sergius Frank ${ }^{2}$, Robert Kumpf ${ }^{5}$, Joachim Schachtner ${ }^{2^{*}}$ and Ernst A. Wimmer ${ }^{1 *}$

\begin{abstract}
Background: The red flour beetle Tribolium castaneum is an emerging insect model organism representing the largest insect order, Coleoptera, which encompasses several serious agricultural and forest pests. Despite the ecological and economic importance of beetles, most insect olfaction studies have so far focused on dipteran, lepidopteran, or hymenopteran systems.

Results: Here, we present the first detailed morphological description of a coleopteran olfactory pathway in combination with genome-wide expression analysis of the relevant gene families involved in chemoreception. Our study revealed that besides the antennae, also the mouthparts are highly involved in olfaction and that their respective contribution is processed separately. In this beetle, olfactory sensory neurons from the mouthparts project to the lobus glomerulatus, a structure so far only characterized in hemimetabolous insects, as well as to a so far non-described unpaired glomerularly organized olfactory neuropil in the gnathal ganglion, which we term the gnathal olfactory center. The high number of functional odorant receptor genes expressed in the mouthparts also supports the importance of the maxillary and labial palps in olfaction of this beetle. Moreover, gustatory perception seems equally distributed between antenna and mouthparts, since the number of expressed gustatory receptors is similar for both organs.

Conclusions: Our analysis of the T. castaneum chemosensory system confirms that olfactory and gustatory perception are not organotopically separated to the antennae and mouthparts, respectively. The identification of additional olfactory processing centers, the lobus glomerulatus and the gnathal olfactory center, is in contrast to the current picture that in holometabolous insects all olfactory inputs allegedly converge in the antennal lobe. These findings indicate that Holometabola have evolved a wider variety of solutions to chemoreception than previously assumed.
\end{abstract}

Keywords: Tribolium castaneum, olfaction, insect, chemoreception, gustation, neuroanatomy, lobus glomerulatus

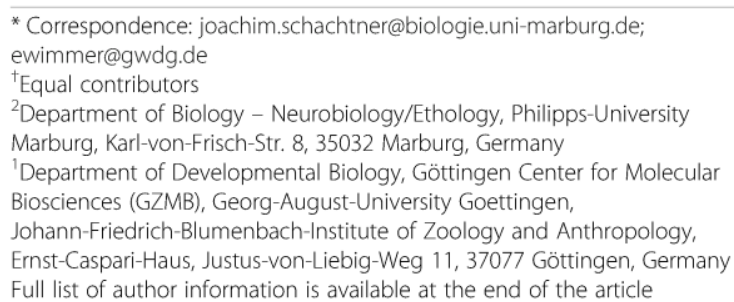




\section{Background}

Insects use chemical cues for most tasks they encounter during their life history. Over long distances, airborne chemical stimuli guide insects to food sources, mates, and places for oviposition [1-6]. Within close range, olfaction as well as gustation are used to discriminate between different food qualities, to avoid toxins or harmful microbes, to communicate intra- or interspecifically, to identify suitable mating partners, and to find appropriate egg-laying sites [6-15]. Because of insects' devastating impact on agriculture and stored food products, as well as their ability to serve as vectors for detrimental diseases, insect olfaction has become an important research field in biology [4].

Chemical signals are typically perceived within specialized antennal and palpal cuticular structures, the olfactory or gustatory sensilla. These chemosensory sensilla form a hollow structure filled with aqueous lymph and harbor the dendritic branches of the chemosensory neurons (CSNs), namely the olfactory (OSNs) or gustatory sensory neurons (GSNs) $[16,17]$. They are divided into several sub-types according to their different morphology [16]. The volatile molecules enter the cavity through wall pores finally to reach and activate the chemoreceptors on the dendrites of the OSNs. To enhance olfactory sensitivity and specificity, odorant binding proteins (OBPs) or potentially chemosensory proteins (CSPs) facilitate the translocation of many, mostly hydrophobic, chemicals through the aqueous lymph [18]. In insects, typically three different receptor families are involved in chemoreception [4]: the ionotropic glutamate-like receptors (IRs) $[19,20]$, the gustatory receptors (GRs) [13, 21], and the odorant receptors (ORs) [22-24]. The IRs are evolutionarily highly conserved chemoreceptors involved in protostome olfaction [19]. They contain three transmembrane domains and form functional heteromers between an odor-specific IR and a co-receptor (IR8a and IR25a) The GRs are seven transmembrane receptors found across arthropods [25-28] whose quaternary structure [13, 29-31], as well as the signal transduction mechanism [32, 33], are still under debate. The typical ORs are seven transmembrane receptors found in pterygote insects [24] that form functional heteromers with the atypical (general) odorant receptor co-receptor (Orco) [22, 23, 34-36]. Their signal transduction mechanism is currently discussed and they may either form an ionotropic receptor complex that is regulated by second messengers or be functional metabotropic receptors $[22,23,34,37-40]$. The described influence of G-proteins and affiliated second messengers on insect olfaction supports both mechanisms [41-47]. Moreover, sensitive pheromone detection requires the OR/Orco complex to interact with a sensory neuron membrane protein (SNMP) related to the scavenger receptor CD36 [48-50]. Besides the perireceptor events involved in effective activation, the high temporal resolution of olfactory reception probably also requires signal termination, which is supposedly mediated by secreted or membrane-bound odorant-degrading enzymes (ODEs) [51-55].

Activation of the described chemoreceptors elicits action potentials in the CSNs that are further transmitted via the antennal nerve to the antennal lobe $(\mathrm{AL})$, the first integration center of the olfactory pathway in the brain, or for GSNs, to the primary gustatory center of the gnathal ganglion (GNG) [56]. The AL of insects consists typically of spherical sub-compartments, the olfactory glomeruli [57]. Usually OSNs express only one typical (specific) OR gene and all antennal OSNs expressing the same typical OR converge into the same olfactory glomerulus, creating a chemotropic map-like representation of chemical coding in the AL [58-60], known as the central dogma of olfaction $[61,62]$. In Drosophila melanogaster, the OR/Orco and IR derived sensory information from the antennae and the maxillary palps is processed in the AL [63], whereas in several hemimetabolous insects, CSNs from the palps converge typically in the lobus glomerulatus (LG), next to but outside the AL [57, 64-66]. In the AL, olfactory information from the OSNs, is processed by a complex network of local interneurons [57, 67-69]. The processed odor information is further relayed by distinct antennal lobe tracts (ALTs) formed by the projection neurons (PNs) to the mushroom body $(\mathrm{MB})$ and the lateral horn (LH) [57, 70]. The MBs are higher-order integration centers for multiple processed sensory information and are responsible for odor discrimination, associative learning, as well as memory storage and retrieval. The LHs receive odor input directly from the ALs or indirectly from the MBs, decode the quality and intensity of the information, and finally trigger immediate odor-driven behavior [71-77].

Despite the evolutionary success and ecological as well as economic importance of beetles [78, 79], little is known on the neuroanatomy, genetics, or biochemistry of their olfactory pathway. Within the Coleoptera, the red flour beetle Tribolium castaneum has become the prime model organism for developmental biology and pest management [80]. With its fully annotated genome [81-83] and the multiple powerful genetic tools - such as systemic RNA interference [84, 85], insertional mutagenesis [86], and transgene-based misexpression systems $[87,88]$ - T. castaneum represents an eligible beetle model organism for olfaction. In the current study, we present a substantial overview of the olfactory pathway in T. castaneum, covering the morphology of the sensilla and the antenna, all major neuropils including $\mathrm{AL}, \mathrm{MB}$, LH, LG, and the gnathal olfactory center (GOC), a previously undescribed glomerularly organized neuropil in the GNG. Additional support for the importance of the 
gnathal input into olfaction is provided by genome-wide expression analysis of gene families involved in chemoreception (e.g., ORs, GRs, IRs, SMNPs, and ODEs) and CSPs and OBPs, which have recently been published [89].

\section{Results}

\section{The Antenna of Tribolium castaneum}

To determine the distribution and number of CSNs, we used immunohistochemistry (IHC) with a cross-reactive antibody against Orco, fluorescent in situ hybridization with an Orco-specific probe, and a transgenic line, EF1-BDsRed, that labels almost all and only CSNs in the adult antenna (see 'Methods' for a detailed characterization). Moreover, we generated an Orco-Gal4 line that partially covers the Orco pattern, which we refer to as the partial OrcoGal4 line (see 'Methods' for a detailed characterization of reagents). These different approaches unequivocally confirm that CSNs are restricted to the distal three segments (9-11) that form the enlarged club of the antenna [90] (Fig. 1a; Additional file 1: Figure S1a; and Additional file 2: Figure S2a). To improve on previous data in respect to the characterization, location, and exact number of antennal sensilla [90], we used in addition to the confocal laserscanning microscopy (CLSM) approaches also scanning electron microscopy (SEM) (Figs. 1b-h and 2a-g). This morphologically verified the presence of chemosensory sensilla exclusively on the three club segments [90], with the highest number and diversity on the apical part of the terminal segment 11 (Fig. 1b-b"; Additional file 3: Figure S3).

Four mechanoreceptive and three chemoreceptive sensilla types could be confirmed by the combination of these techniques (Fig. 1b-b") and the respective number of contained CSNs was identified. The mechanoreceptive sensilla include the spatulate bristles (SpaB; Fig. 1d-d"'), the mechanosensilla trichoidea (mSTri; Fig. 1e-e'"'), the sensilla campaniformes (SCam; Fig. 1b"), and the sensilla chaetica (SCha; Fig. 1c-c"'), which are the most dominant sensilla type present on the lateral sites of all $11 \mathrm{seg}$ ments (Fig. 1a). The chemoreceptive sensilla subdivide into chemo-sensilla trichoidea (cSTri, Fig. 1f-f"'), sensilla basiconica (SBas; Fig. 1g-g"'"), and sensilla coeloconica (SCoe, Fig. 1h-h"'). For the chemoreceptive sensilla, segments 9 and 10 carry mostly SBas (about 15) arranged in an axial ring at the apical edge of each segment (Fig. 2f, g) and two SCoe (Fig. 2f, g'), whereas the terminal segment 11 harbors SBas (about 25), some SCoe (about 7), and many cSTri (about 87) (Fig. 1b-b"). A detailed analysis of the number and distribution of the different sensilla types in males and females revealed no sexual dimorphism (Additional file 3: Figure S3).

The number of CSNs per antenna was estimated based on the number of CSNs per sensillum or prong and the number of the respective sensilla per antenna. cSTri contain typically one Orco-immunoreactive OSN
(Additional file 1: Figure S1b; Additional file 3: Figure $\mathrm{S} 3 \mathrm{i})$. This type of sensilla is known for its pheromone receiving abilities in Lepidoptera [91-93] and had been described as olfactory sensilla in D. melanogaster [59] and Culex quinquefasciatus [94]. SBas of T. castaneum consist of up to five prongs (Fig. 2a-e) like other Tenebrionidae $[90,95]$. Each prong harbors about six CSNs (Additional file 3: Figure S3i) - the same number as in Tenebrio molitor [96]. Of them, four or five can be considered olfactory based on Orco-immunoreactivity (Additional file 1: Figure S1c). Findings in Tribolium brevicornis [95] suggest an additional gustatory function of SBas, leading to the conclusion that the SBas are bimodal chemosensilla. Because of this constant number of CSNs per prong and the shared lymphatic space (Fig. 1g-g"), we propose that multiple pronged SBas are derived from a fusion of single sensilla. Nonetheless, we refer to and count multiple pronged SBas as a single sensillum independent of the number of prongs. SCoe contain three CSNs (Additional file 3: Figure S3i) without Orco-immunoreactivity (Additional file 1: Figure S1d). The SCoe in T. castaneum might therefore harbor IRs as shown in D. melanogaster $[19,97]$. Altogether, we found on each antenna about 100 prongs of SBas with six CSNs each, 87 cSTri with one CSN, and 11 SCoe with about three CSNs (Additional file 3: Figure S3). This leads to a total number of about 720 CSNs per antenna of T. castaneum.

\section{Anatomy of the olfactory pathway in the red flour beetle brain \\ Antennal projections}

To get an impression of the innervation pattern of chemosensory neuropils, we performed antennal and palpal backfills. Backfills via the antennal nerve labeled the ipsilateral AL (Fig. 3a; Additional file 4: Movie S1; Additional file 5: Movie S2; Additional file 6: Figure S4), the antennal mechanosensory and motor center (AMMC) (Fig. 3b), as well as a distinct area in the GNG (Fig. 3c). While this ipsilateral restriction is common in many insects [57], it is in contrast to D. melanogaster and Ceratitis capitata, where the majority of OSNs innervate the ipsi- and contralateral sides $[59,98]$. The antennal backfills labeled all AL glomeruli except one, which is the only glomerulus labeled by backfills of the maxillary palp via the GNG (Fig. 3a; Additional file 5: Movie S2). This resembles the situation in Lepidoptera, where $\mathrm{CO}_{2}$ responsive CSNs from the palp project into a single AL glomerulus devoid of antennal innervation [99]. The descending antennal projections into the GNG (Fig. 3c) are not labeled in the partial Orco-Gal4/UASDsRed line and might therefore be from gustatory or mechanosensory neurons, as described in Periplaneta americana and Locusta migratoria [100, 101]. 

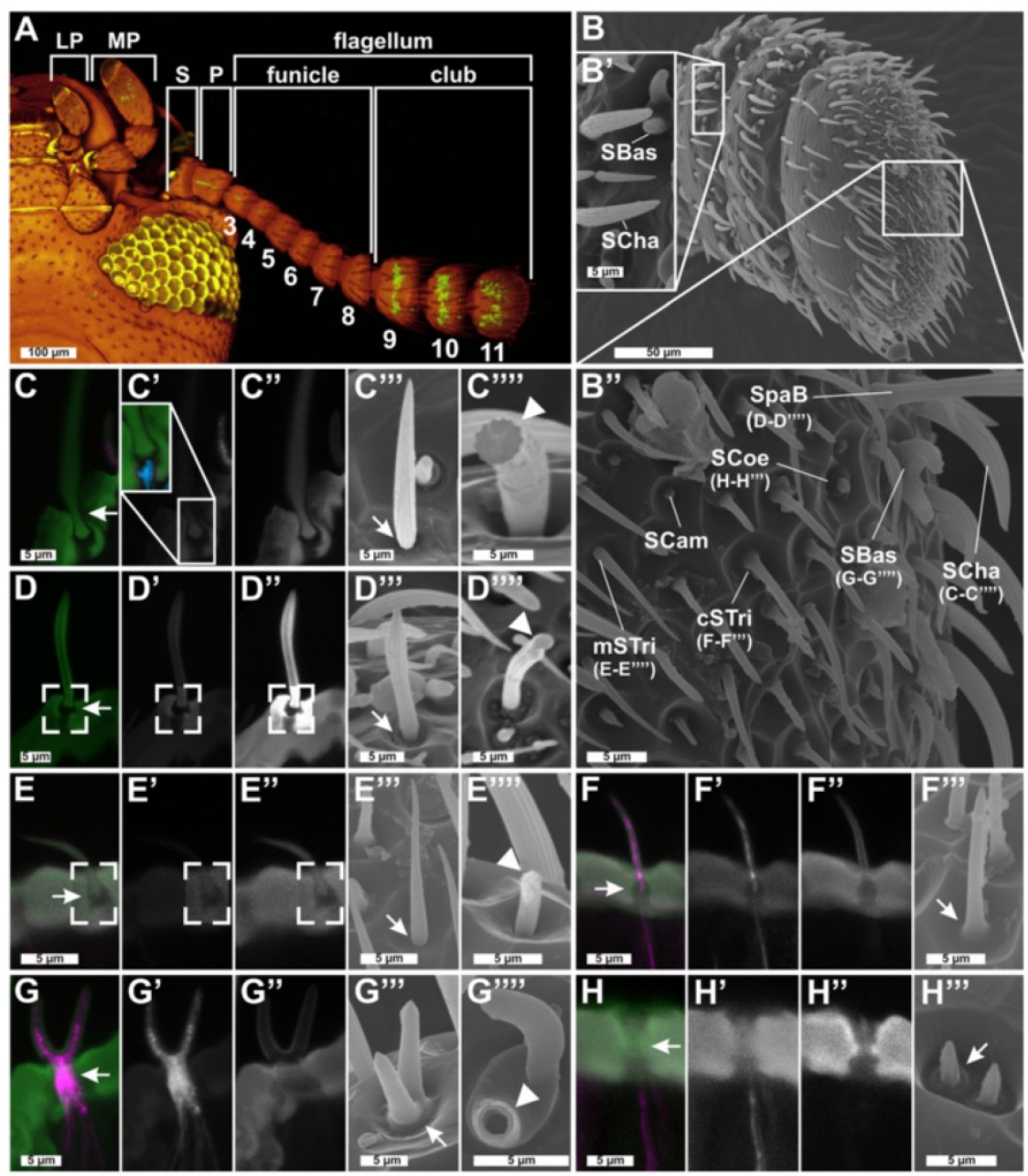

Fig. 1 Sensilla types and distribution on Tribolium castaneum antennae I. a Chemosensory sensilla are restricted to the distal three segments (9-11) of the T. castaneum antenna, which is composed of scape (S), pedicel $(P)$, and flagellum, and the last labial palp (LP) and maxillary palp (MP) segment. CLSM-stack voltex projection of a transgenic beetle head (ventral view, green: partial Orco-Gal4/UAS-tGFP; yellowish eye, brownish cuticle: autofluorescence). b-b" SEM images of the club segments with close-up of segments 9 (b') and $11\left(\mathbf{b}^{\prime \prime}\right)$. Single sensilla: CLSM maximum intensity projection overlays (c-h) of antibodyenhanced EF1-B-DsRed reporter signal (magenta, $\mathbf{c}^{\prime}-\mathbf{h}^{\prime}$ ) and cuticle autofluorescence (green, $\mathbf{c}^{\prime \prime}-\mathbf{h}$ "). $\mathbf{c}^{\prime \prime \prime}-\mathbf{h}$ '" SEM analysis. Mechanoreceptive sensilla: SCam are small, smooth, and dome-shaped sensilla restricted to segment 11 (Additional file 3: Figure S3a); SCha - previously described as spines [90] - are longitudinally corrugated, connected to a neuron at the socket (c'; blue), jointed (c'"; arrow), and solid (c'"'; arrowhead). d-d'"' SpaB - in T. brevicornis called sensilla squamiformium [95] - resemble modified (slightly thicker tip) SCha [96] restricted to segment 11 (Additional file 3: Figure S3b). e-e'"' mSTri (structurally similar to SCha but smaller more hair-like appearance) have previously been described in other species [24, 244]. CLSM analysis showed joint-like structures at the base (c-e, $\mathbf{c}^{\prime \prime}-\mathbf{e}^{\prime \prime}$, open squares) of the mechanoreceptive sensilla and SEM revealed a small gap at their base (c'"--e'", arrow). Chemoreceptive sensilla: $\mathbf{f}-\mathbf{f}^{\prime \prime}$ cSTri are hair-like structures restricted to segment 11 (Additional file 3: Figure S3d) with a rounded tip and a smooth transition of the base; $\mathbf{g}-\mathbf{g}^{\prime \prime \prime \prime}$ SBas are smooth-surfaced pegs with rounded tips and smooth transitions at the base ( $\mathbf{g}^{\prime \prime \prime} ;$ arrow). $\mathbf{h}-\mathbf{h}^{\prime \prime \prime}$ SCoe are short and corrugated, and their transition into the antennal cuticle shows a typical elevation ( $\left.\mathbf{b}^{\prime \prime}, \mathbf{h}^{\prime \prime \prime}\right)$. All chemoreceptive sensilla $\left(\mathbf{f}, \mathbf{g}, \mathbf{f}-\mathbf{h}^{\prime}\right)$ house dendritic branches of CSNs labeled by DsRed. The close-up in c' shows a non-CSN fiber entering only the base of a SCha labeled with phalloidin (blue).

Chemoreceptive sensilla show a smooth transition into the antennal cuticle (f"'-h'", arrow). Whereas all mechanoreceptive sensilla are solid cuticular structures (fractured in c'"'--'e'"'), chemoreceptive SBas appear hollow (fractured in $\mathbf{g}^{\prime \prime \prime \prime}$ ). CLSM confocal laser-scanning microscopy, CSN chemosensory neuron, CSTri chemosensilla trichoidea, LP labial palp, MP maxillary palp, mSTri mechanosensilla trichoidea, P pedicel, S scape, SBas sensilla basiconica, SCam sensilla campaniformes, SCha sensilla chaetica, SCoe sensilla coeloconica, SEM scanning electron microscopy, SpaB spaculate bristle

\section{Antennal lobe}

For the AL of freshly eclosed adults, about 70 distinguishable olfactory glomeruli have been previously described using a synapsin antibody [102]. To evaluate the glomeruli number in ALs of beetles, 7 days after adult eclosion, we improved the analysis by deconvolution as well as using an additional antiserum against tachykininrelated peptides (TKRPs), which distinctly labels also 


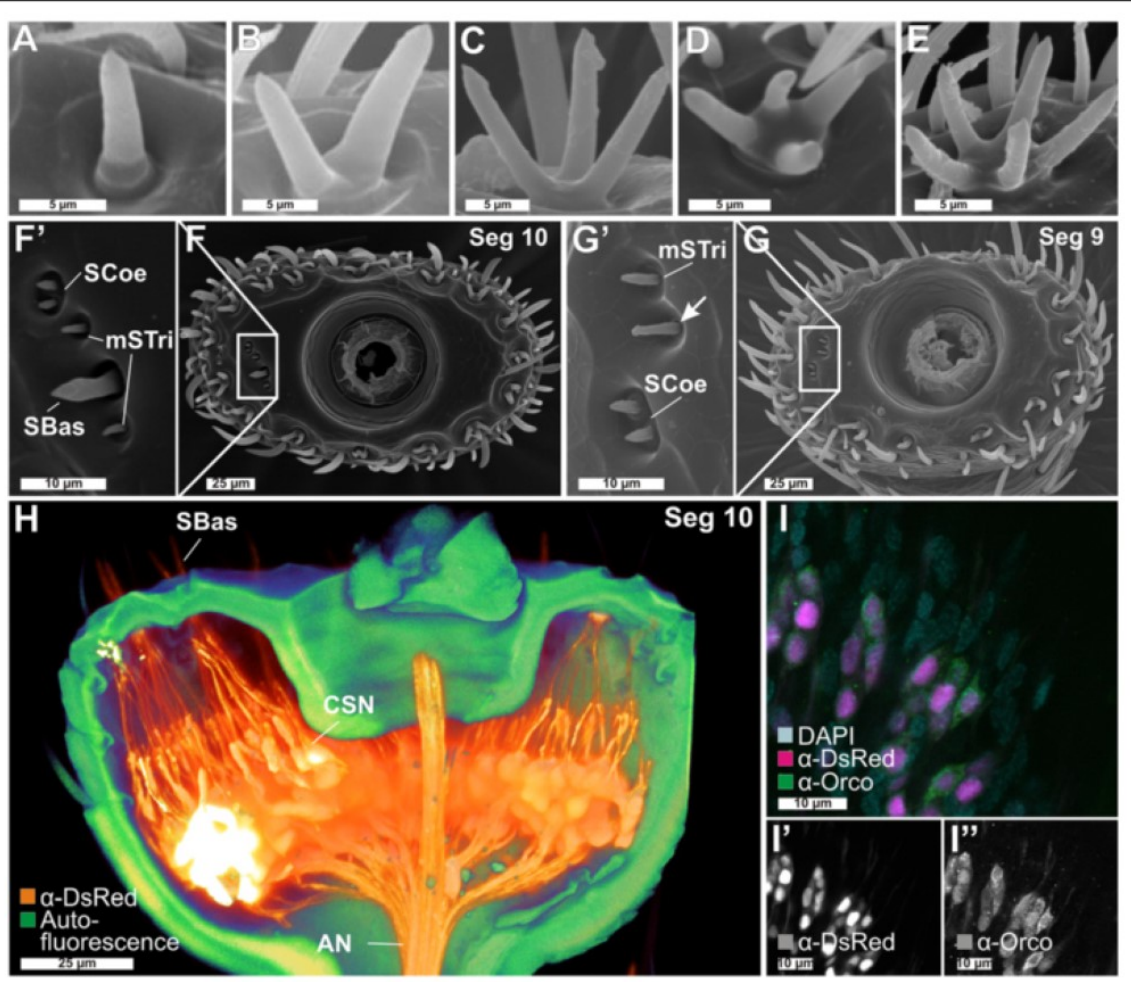

Fig. 2 Sensilla types and distribution on Tribolium castaneum antennae II. a-e SEM images of SBas with one to five prongs. $\mathbf{f}$, $\mathbf{f}$ SEM image of the tenth segment of the antenna with a close-up of the lateral corner (f') containing SCoe, SBas, and $m S T r i . \mathbf{g}$, $\mathbf{g}$ ' SEM image of the ninth segment with a close-up of the lateral corner ( $\mathbf{g}^{\prime}$ ) showing SCoe and mSTri. Chemoreceptive SCoe were previously described as "minute spicule-like sensilla trichoidea" [90], are relatively rare (Additional file 3: Figure S3e), and located besides the lateral corners of segments 9 and 10 (f', g') mostly at the apical side of segment 11 (Fig. 1 b"). Chemoreceptive SBas are arranged in an axial ring at the distal margins of all three club segments (f, $\mathbf{g}$, Fig. 1b-b"). For mechanoreceptive $m S T r$, we identified about 37 on the apical side of segment 11 (Fig. 1b") and four in lateral corners of segments 9 and 10 (f, f', g, $\mathbf{g}^{\prime}$, and Additional file 3: Figure S3c, h). $\mathbf{h}$ Voltex projection based on a CLSM image stack of the tenth segment from the EF1-B-DsRed line displaying CSNs (orange) and autofluorescence of the cuticle (green). The dendrites of the CSNs converge into the SBas (on average, six per prong), while the axons unite at the center of the segment and join the antennal nerve (AN). i-i" Overlay of the signals of the DsRed reporter (magenta, i') and the Orco antibody (green, $\mathbf{i}^{\prime \prime}$ ) together with DAPI staining (light blue) in the EFT-B-DsRed line, demonstrating a high level of colocalization between DsRed and Orco in segments 9 and 10, but not in 11, where some DsRed-immunoreactive CSNs are spared (compare with Additional file 1: Figure S1a). AN antennal nerve, CLSM confocal laser-scanning microscopy, CSN chemosensory neuron, mSTri mechanosensilla trichoidea, Orco odorant receptor co-receptor, SBas sensilla basiconica, SCoe sensilla coeloconica, Seg segment, SEM scanning electron microscopy

densely packed glomeruli [103]. This more advanced analysis resulted in the $3 \mathrm{D}$ reconstruction of about 90 glomeruli per AL with no obvious sexual dimorphism (females: mean 89.2, standard deviation or $\mathrm{SD}=4.9, n=$ 5; males: mean 89.4, SD, 7.6, $n=5$ ).

\section{Palpal projections into accessory olfactory centers}

Whole mouthparts or maxillary palp backfills (Fig. 3d, e) revealed besides the already mentioned single AL glomerulus, innervation of three distinct neuropil areas: an unpaired glomerular organized neuropil in the GNG, the primary gustatory center also in the GNG [104], as well as an area near the $\mathrm{AL}$, resembling the $\mathrm{LG}$ of hemimetabolous insects $[57,105]$. The unpaired neuropil located n-anterodorsal in the GNG consists of 30 to 40 glomeruli (Fig. 3d, inset), which are all innervated from both sides of the mouthparts. This neuropil is also labeled by the partial Orco-Gal4/UAS-DsRed line (Fig. 3f; Additional file 7: Movie S3), which indicates innervation by OSNs originating in the maxillary or labial palps (Figs. 1a, 3f, 4a", 4b"; Additional file 2: Figure S2f) that project via two tracts into the GNG. This neuropil, therefore, represents an olfactory processing center in the GNG that has to our knowledge never been described before and we term the gnathal olfactory center (GOC). Some of the fibers labeled by the palpal backfills, as well as the partial Orco-Gal4/UAS-DsRed line pass through the GOC, ascend via the neck connectives, and terminate ipsilaterally in an area medioventral to the AL (Fig. 3d), resembling the LG, which to date had only been described in hemimetabolous insects [57, 105]. Since the position, innervation, and glomerularly 

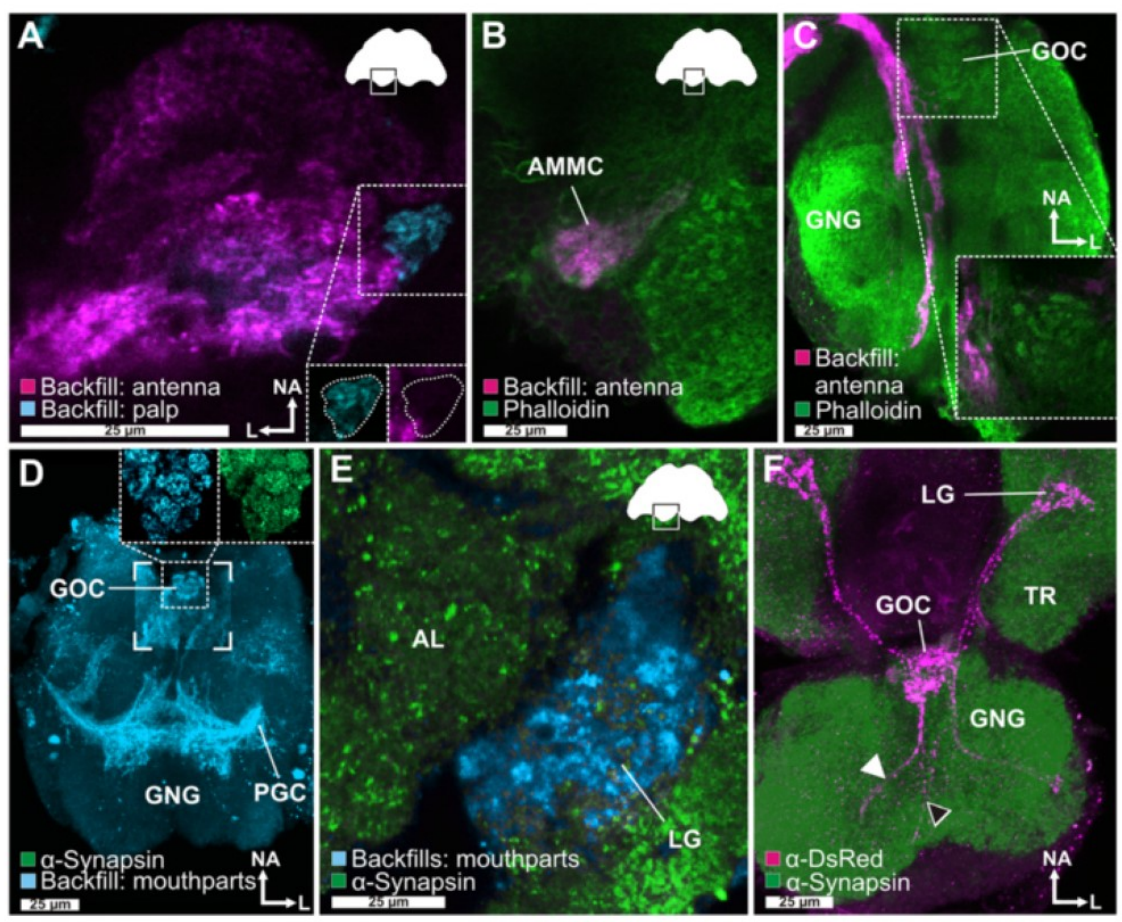

Fig. 3 The central olfactory pathway of $T$. castaneum. a Backfill of one antenna (magenta) stains all glomeruli in the ipsilateral antennal lobe (AL) except one. This glomerulus is exclusively labeled by a backfill of a maxillary palp (cyan). $\mathbf{b}$ In addition to the AL glomeruli, backfilling (magenta) of one antenna labeled the ipsilateral antennal mechanosensory and motor center (AMMC), located n-dorsally to the $A L, \mathbf{c}$ as well as descending fibers to the gnathal ganglion (GNG). d Maximum intensity projection of the backfills of mouthparts (cyan) shows massive innervation of the GNG including the gnathal olfactory center (GOC) (magnified in the inset) and the primary gustatory center (PGC). e Backfill of the mouthparts (cyan) revealed in the cerebral ganglion beside innervation of a single ipsilateral $A L$ glomerulus also projections in the ipsilateral lobus glomerulatus ( $L G$ ). f Reporter expression of the partial Orco-Gal4/UAS-DsRed line (magenta) revealed two paired input tracts (black and white arrowheads) from the maxillary (white arrowhead) and labial palps (black arrowhead) that converge in a medial and n-anterodorsally located glomerular area, the GOC, and ascend to a microglomerularly organized area, the LG. See also Additional file 7: Movie S3. Orientation bars in (a) also apply for (b) and (e). AL antennal lobe, AMMC antennal mechanosensory and motor center, GNG gnathal ganglion, GOC gnathal olfactory center, L lateral, $L G$ lobus glomerulatus, NA neuroaxis-anterior, $P G C$ primary gustatory center, $T R$ tritocerebrum

organized structure of this paired neuropil in T. castaneum is similar to the LG in cockroach, locust, and silverfish [57, 64-66], we refer to it as LG. In summary, our data suggest that in $T$. castaneum, odor information from the antennae and the mouthparts are processed separately. It appears that OSNs from the mouthparts do not project into the AL but into the GOC and the LG.

\section{Projection neurons}

Dye injections into the AL of adult T. castaneum revealed three ALTs formed by the PNs (Fig. 5), exclusively in the ipsilateral hemisphere. The most prominent tract, the medial antennal lobe tract (mALT), connects the AL with the calyx (CA) of the MB and the LH. The mediolateral antennal lobe tract (mlALT) passes the region near the MB spur without forming sub-branches (Fig. 5) and further projects to the LH. The lateral antennal lobe tract (IALT) projects directly to the most n-posterior part of the LH. We could not observe any obvious direct projections of the mlALT and the IALT to the CA. However, since they possibly overlap with trajectories of the mALT fibers from the CA to the LH [106], we cannot exclude their existence, as described for other holometabolous insects [70]. Previously only the mALT had been clearly identified in Coleoptera and the existence of a mlALT had only been presumed [70]. Our results indicate that three ALTs are a common feature among most holometabolous insects, including beetles.

\section{Mushroom body}

The detailed architecture of the MB of T. castaneum is described in [103]. The CA is innervated by the mALT (Fig. 5) and microglomerularly organized as indicated by phalloidin or synapsin antibody stainings (Fig. 5, inset). This is similar to several insects including Apis mellifera $[107,108]$ and D. melanogaster $[109,110]$ and suggests a comparable wiring with the PNs. The Kenyon cells (KCs) were identified in DAPI (4',6-diamidino-2- 

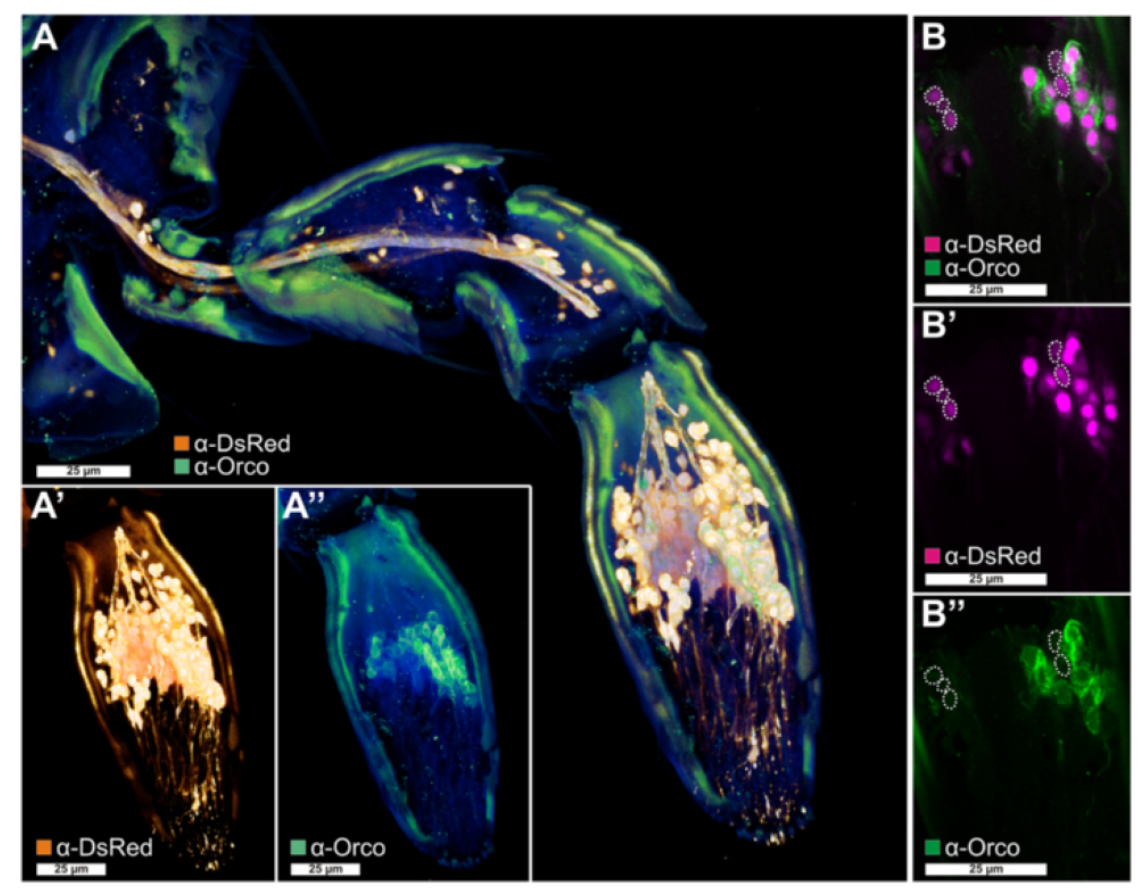

Fig. 4 Orco-immunoreactive sensory neurons in the maxillary palp. a Voltex projection of a CLSM-stack showing antibody enhanced reporter expression of the EFT-B-DsRed line (a', orange) and Orco-immunoreactive cells (a", green) in a halved maxillary palp. b-b" Single optical section of (a) showing partial colocalization of Orco immunoreactivity and the reporter expression of the EFT-B-DsRed line (magenta). Dotted lines in (b) highlight reporter-expressing cells that are not Orco-immunoreactive. CLSM confocal laser-scanning microscopy, Orco odorant receptor co-receptor

phenylindole) stainings based on their smaller and brighter stained nuclei [103]. The number of about 2700 KCs was determined by interpolation of volumetric data as well as by counting of the stained nuclei using MorphoGraphX [111]. Both procedures resulted in comparable numbers with the interpolation of 13 CAs from seven animals estimating about $2800 \mathrm{KCs}$ (2795; SD: 214) and the counting of nine CAs from five specimen indicating approximately $2600 \mathrm{KCs}$ (2613; SD: 204) per MB.

\section{Genome-wide expression analysis of genes involved in chemoreception in $T$. castaneum}

The fully sequenced genome of $T$. castaneum [81-83] led to the annotation of the major gene families involved in chemoreception. Based on genome data and computational gene predictions, the OBPs [112], CSPs [113], IRs [114], GRs [81], ORs [115], and SNMPs [116, 117] were annotated, but only for the ORs was a RT-PCRbased expression analysis performed [115]. To validate or correct the predicted gene models of these gene family members and to determine their tissue-specific expression, we performed transcriptome analyses of adult male and female antennae, heads (without antennae, but including mouthparts), mouthparts (the part of the head capsule, anterior to the antennal bases), legs, and bodies (without legs and head). In addition, we identified potential ODEs, as well as orthologs from further genes described to be involved in D. melanogaster olfaction. The detailed analysis of the OBPs and CSPs has already been published [89] and revealed that the majority of the classic OBPs and antenna binding proteins II (ABP II) seem to be involved in chemoreception while only a few of the minus- $C$ OBPs (C-OBPs) and CSPs are enriched in antenna or mouthparts. The following results are based on this same set of transcriptome data (GEO accession number: GSE63162 (http://www.ncbi.nlm.nih.gov/geo/query/acc.cgi?acc=GSE63 162 [118]). Like OBPs and CSPs [89], also for the genes presented here, no significant differences on the expression level between male and female antenna samples were identified (Fig. 6). Therefore, the female and male antenna samples can serve as biological replicates and indicate that reads above 0.1 reads per kilobase per million (RPKM) are reproducible (Fig. 6). However, to minimize the rate of potential false positives in our description, we considered only genes with RPKM $\geq 0.5$ as expressed. We are aware that this might lead to an underestimation of the expressed gene numbers for each class of genes. Since it is impossible to determine the exact number of genes that are functionally involved in chemoreception based only on transcriptomic expression analyses, we always present two values for expressed genes, one based on RPKM $\geq 0.5$ and the other defined by 


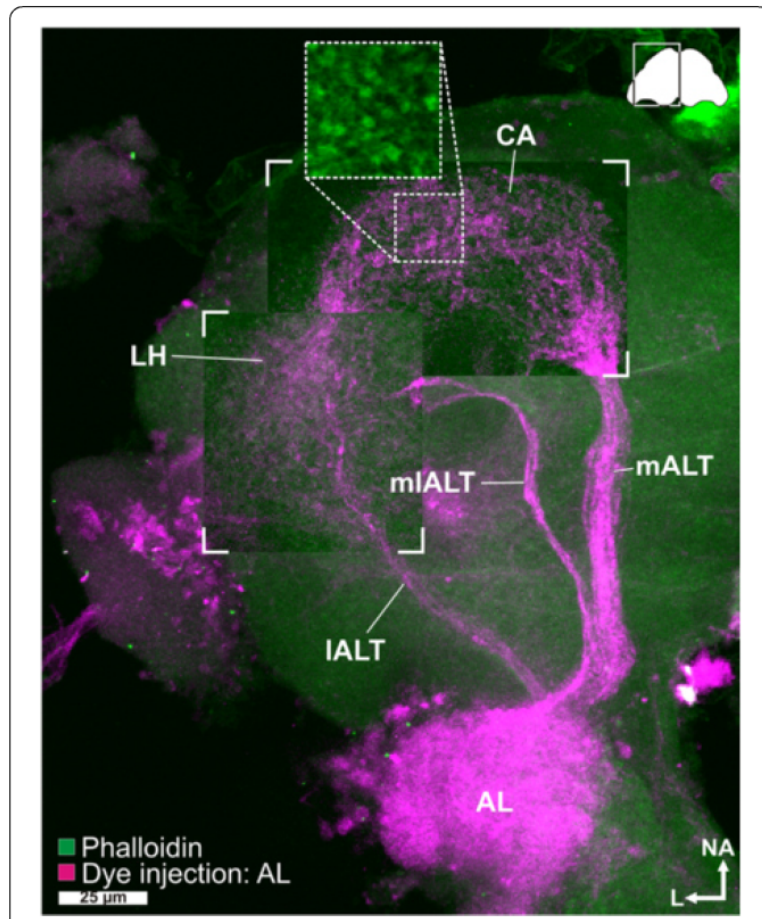

Fig. 5 Antennal lobe tracts. Maximum intensity projection of a CLSM image stack after dye injection into the AL (magenta) revealed three antennal lobe tracts - the medial ( $m A L T)$, mediolateral ( $m / A L T)$, and the lateral antennal lobe tract (IALT) - as well as the calyx $(C A)$ and the lateral horn $(L H)$. In the $C A$, most fibers from the mALT form microglomeruli (inset obtained from another preparation). The staining in the optical lobe is an artifact caused by diffusion of the dye during application. Phalloidin counterstaining in green. AL antennal lobe, ALT antennal lobe tracts, CA calyx, CLSM confocal laser-scanning microscopy, IALT lateral antennal lobe tract, $L H$ lateral horn, MALT mediolateral lobe tract, MIALT mediolateral lobe tract

statistical analysis as significantly enriched over body. All raw values and the re-annotated gene models are summarized in Additional file 8.

\section{Tissue-specific expression of ionotropic glutamate-like receptors}

The RNAseq based revision of the 23 previously annotated IRs [114] confirmed the sequences of three open reading frames (ORFs); 17 had to be modified, two were incompletely covered by reads, and for a single one, no expression was detected (color coded in Additional file 8: Table S1, column B). In antennae, 16 of the IRs were significantly enriched compared to body (Fig. 7; Additional file 9: Figure S5a). In the mouthparts, five IRs are expressed, two are significantly enriched.

Comparing expression profiles of the IRs from $T$. castaneum, D. melanogaster, and Anopheles gambiae confirmed the antennal specific expression, as well as the high degree of phylogenetic conservation of the antennal IRs (Fig. 8; Additional file 10: Figure S6; highlighted in yellow) as proposed [114]. In contrast, the divergent IRs are non-antennal specifically expressed and are highly radiated within species clades as previously shown or predicted [114]. T. castaneum has a lower number of IRs compared to D. melanogaster and An. gambiae, due to lesser expansions of divergent IRs, but maintains the basic repertoire of antennal IRs (Fig. 8; highlighted in yellow). The homologs of IR25a, IR93a, and IR40a, which are necessary for humidity perception in D. melanogaster [119], are significantly enriched in antennae. IR40a is exclusively expressed in antennae, which correlates with the essential role of antennae in $T$. castaneum hygro-perception [90]. The homolog of the highly sensitive salt receptor and possible co-receptor IR76b $[20,120]$ is significantly enriched in antennae, mouthparts, and legs, while the co-receptors IR8a and IR25a [20] are highly expressed in all tissues of T. castaneum (Fig. 7).

\section{Tissue-specific expression of gustatory receptors}

Of the 220 previously annotated GRs [81], only 207 genes had available gene models [82, 83]. Our transcriptome analysis verified the ORFs of 58 GRs, showed slight differences for $20 \mathrm{GRs}$, but did not or only incompletely cover 129 GRs (Additional file 8: Table S1, column B). In the antennae, 62 GRs are expressed, with 34 being significantly enriched and 10 being antennal-specific. Of the 69 mouthpart-expressed GRs, 36 are significantly enriched and 19 exclusive. Seventeen GRs are significantly enriched in both antenna and mouthparts. In legs, 18 GRs are expressed with three being significantly enriched (Fig. 9; Additional file 11: Figure S7a).

The phylogenetic comparison of the GRs in $T$. castaneum, D. melanogaster, and An. gambiae (Fig. 10; Additional file 12: Figure S8) confirmed that only the $\mathrm{CO}_{2}$ receptors (highlighted in orange) are highly conserved [121]. The other GRs seem to have undergone independent radiation during the transition to $T$. castaneum: e.g. the sugar receptor-related branch (highlighted in light yellow) contains 16 genes [122], twice the number compared to the two chosen dipterans. In addition, the single fructose receptor (highlighted in grey) found in D. melanogaster and An. gambiae is represented by eight homologs in T. castaneum. The remaining 180 GRs belong to several $T$. castaneum-specific expansion groups. Specific orthologs to the known bitter receptors of D. melanogaster [13] as well as to the thermo-sensitive DmelGR28bD [123] cannot be predicted based on our phylogenetic analysis.

Like other insects $[121,124,125], T$. castaneum has three $\mathrm{CO}_{2}$ receptors (TcasGR1, TcasGR2, and TcasGR3), while $D$. melanogaster has only two that form functional heteromers $[126,127]$. In $T$. castaneum, the expression of the $\mathrm{CO}_{2}$ receptors is not restricted to one of the chemosensory organs with TcasGR2 and TcasGR3 being significantly enriched in 


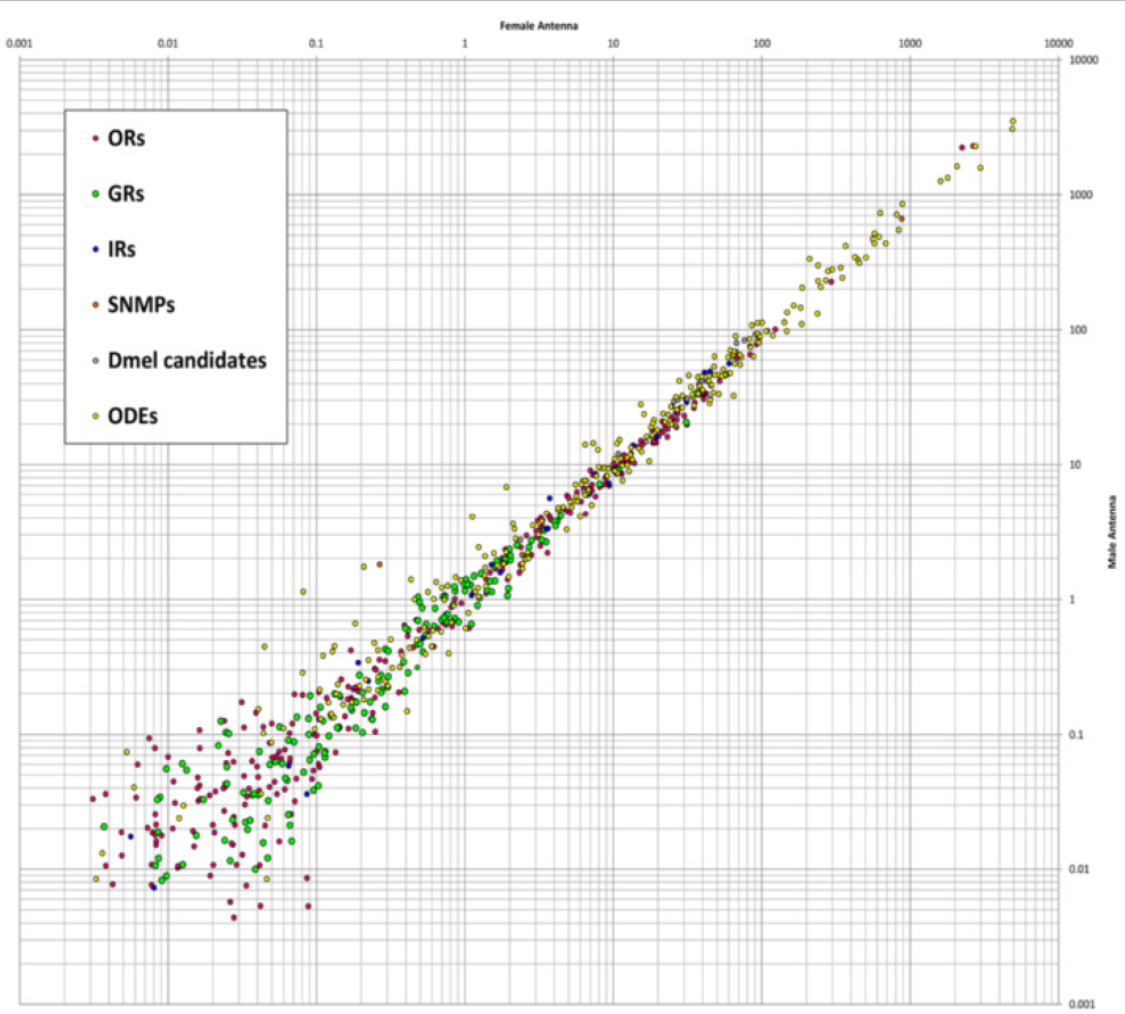

Fig. 6 Comparison of expression levels in male and female antenna. Comparison of expression levels of odorant receptors (ORs, magenta), gustatory receptors (GRs, green), ionotropic glutamate-like receptors (IRs, blue), sensory neuron membrane proteins (SNMPs, orange), orthologous of candidates obtained from D. melanogaster (Dmel candidates, grey) and potential odorant degrading enzymes (ODEs, yellow) in male and female antennae. Average values based on two male and three female antennal samples. Scatter plot of the RPKM values. Dmel D. melanogaster, GRs gustatory receptors, IRs ionotropic glutamate-like receptors, ODEs odorant degrading enzymes, ORs odorant receptors, RPKM reads per kilobase per million, SNMPS sensory neuron membrane proteins

antennae but also being expressed together with TcasGR1 in the mouthparts (Fig. 9; highlighted in orange). This dual input is in contrast to but combines both the expression of the three $A n$. gambiae $\mathrm{CO}_{2}$ receptors that are restricted to the maxillary palps $[128,129]$, as well as the two D. melanogaster $\mathrm{CO}_{2}$ receptors that are mainly expressed in the antennae $[126,127,130]$.

The presence of GRs on insect antenna had previously been postulated based on physiological response to sugars [95, 131-133] and was identified by antennal expression analysis [124, 127, 128, 134-136]. Our interspecies comparison (Fig. 10) confirms the antennal enrichment of several GRs in the two analyzed dipterans. However, the high number of 34 significantly enriched GRs in the antenna of $T$. castaneum is unusual, but reflects the increased total number of GRs in this species. Interestingly, the GRs of T. castaneum are present in both antenna and mouthparts at similar numbers and expression levels (Fig. 9; Additional file 11: Figure S7a).

\section{Tissue-specific expression of odorant receptors}

Of the 341 previously annotated OR sequences [115], we could re-analyze 337 based on our RNAseq data. This revision confirmed 97, and 22 were re-annotated reviving eight previously indicated pseudogenes [115], namely TcasOR2, TcasOR18, TcasOR19, TcasOR22, TcasOR85, TcasOR99, TcasOR104, and TcasOR122. Moreover, 219 genes were not or only partially covered by our transcriptome data (color coded in Additional file 8: Table S1, column B). Over all samples, 170 ORs are expressed (Fig. 11; Additional file 13: Figure S9a). In antennae, 129 ORs are expressed, with 92 being significantly enriched and 99 exclusive. In the mouthparts, 49 ORs are expressed, with 28 being significantly enriched and 27 exclusive. In addition, 16 of the significantly mouthpart-enriched ORs are not enriched in the antenna (Fig. 11). The expression of typical ORs in the mouthparts is consistent with the high expression of Orco in this tissue (Figs. 1a and 4) and with observations in other insect species [128, 129, 137, 138]. In legs, ten ORs are expressed (Additional file 13: Figure 


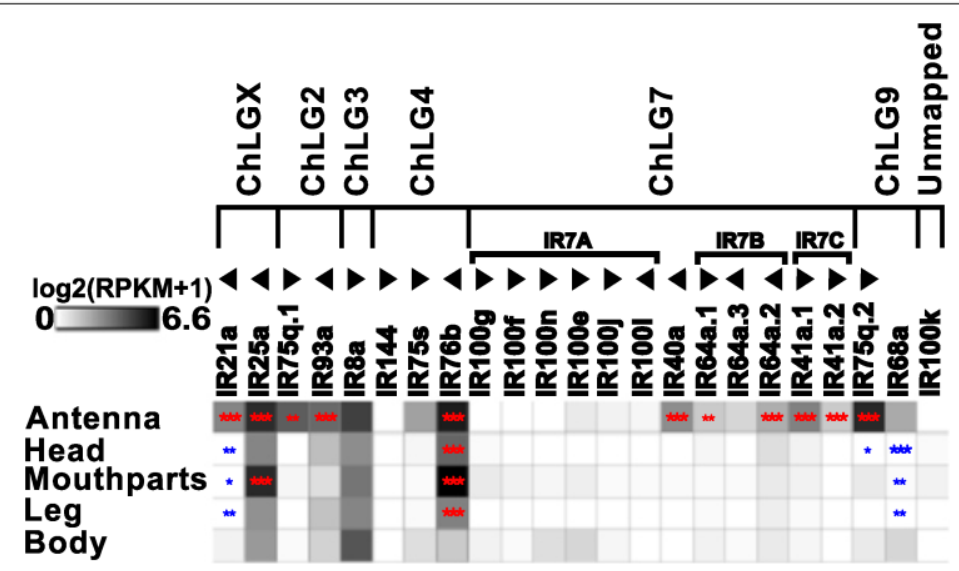

Fig. 7 Expression of $T$. castaneum ionotropic glutamate-like receptors (IRs). Heat map showing the expression level of the 23 IRs as a log, $[R P K M+$ 1] value in different tissues [adult antennae, head (missing antennae but including mouthparts), mouthparts, legs, and body]. The candidates are ordered according to their chromosomal localization (Additional file 9: Figure S5b). Horizontal brackets above indicate clustering in the genome. The arrowheads represent the orientation of the open reading frame. The expression levels are represented by a greyscale with highest shown expression levels labeled black. The asterisks mark statistically significantly differentially expressed genes compared to body (based on biological replicates of five antennal, two head, three mouthpart, two leg, and two body samples). The red asterisks represent up- and the blue down-regulation ( $p$ values adjusted are ${ }^{*}<0.05,{ }^{* *}<0.01$, and ${ }^{* *}<0.001$ ). IR ionotropic glutamate-like receptor, RPKM reads per kilobase per million

S9a) but only one, namely TcasOR127, is statistically enriched (Fig. 9).

The phylogenetic comparison of OR expression patterns in T. castaneum, D. melanogaster, and An. gambiae (Fig. 12; Additional file 14: Figure S10) revealed that the atypical odorant co-receptor Orco (in T. castaneum previously called TcOR1 [115]) is the highest expressed OR in all tissues of all three species. In T. castaneum, Orco is expressed highest in antenna, followed by mouthparts. Orco is the only OR of T. castaneum with clear orthologs in dipterans $[115,139]$. The high expression levels, the distribution, and the evolutionary conservation of Orco are consistent with its ancestral origin [24] and its outstanding role as a chaperone and co-receptor, forming functional heteromers with all typical ORs $[140,141]$.

The exceptional high number of typical ORs (Fig. 12) in $T$. castaneum is the result of large gene radiations within the coleopteran and tenebrionid lineages [136], which were previously subdivided into six expansion groups (Fig. 12) [115]. Expansion groups 1, 2, and 3 are conserved in other coleopterans [136] and are mainly expressed in antennae. The ORs of the expansion groups 4,5 , and 6 are highly derived, have no described homologs in other insects, and their expression is unusually often mouthpart-enriched (Fig. 11; grey lettering). This is consistent with the elaborated role of the mouthparts in T. castaneum olfaction. Specific orthologs to deorphanized ORs of $D$. melanogaster [142] cannot be predicted based on our phylogenetic analysis.

\section{Identification and expression of potential odorant degrading enzymes}

The genome of $T$. castaneum contains 15 aldehyde dehydrogenases (ALDHs) (Fig. 13 and Additional file 15) with two of them being significantly enriched, but not exclusively expressed in antenna. We found four predicted genes encoding aldehyde oxidases (ALOXs) with one being highly enriched in antennae and mouthparts, which, in contrast to ALOX ODEs from lepidopterans [143-145], does not encode a signal peptide (Fig. 13). Five of the 54 identified carboxylesterases (CESs) are significantly enriched in antenna, with two of them also in the mouthparts. Two other CESs are significantly enriched exclusively in the mouthparts. Five of these seven candidates show a predicted signal peptide for secretion (Fig. 13). TcasCESXA shares sequence similarities with $D$. melanogaster Est6, and TcasCES7J with DmelJHEdup, with both D. melanogaster homologs having previously been identified as ODE candidates $[54,55]$. TcasCES10C is expressed highest in antennae and related to a pheromone degrading enzyme from the Japanese beetle, Popillia japonica [146]. We identified six epoxide hydrolases (EHs), which are supposed to be membrane bound ODEs [147], with one being significantly enriched in antennae and having a predicted signal peptide (Fig. 13). The glutathione S-transferases (GSTs) of T. castaneum had already been annotated [148]. The revision confirmed most gene models, only TcasGSTd2 and TcasMGST2 had to be modified (available in Additional file 8: Table S1). Eight of the 41 GSTs are significantly enriched in antennae, with 


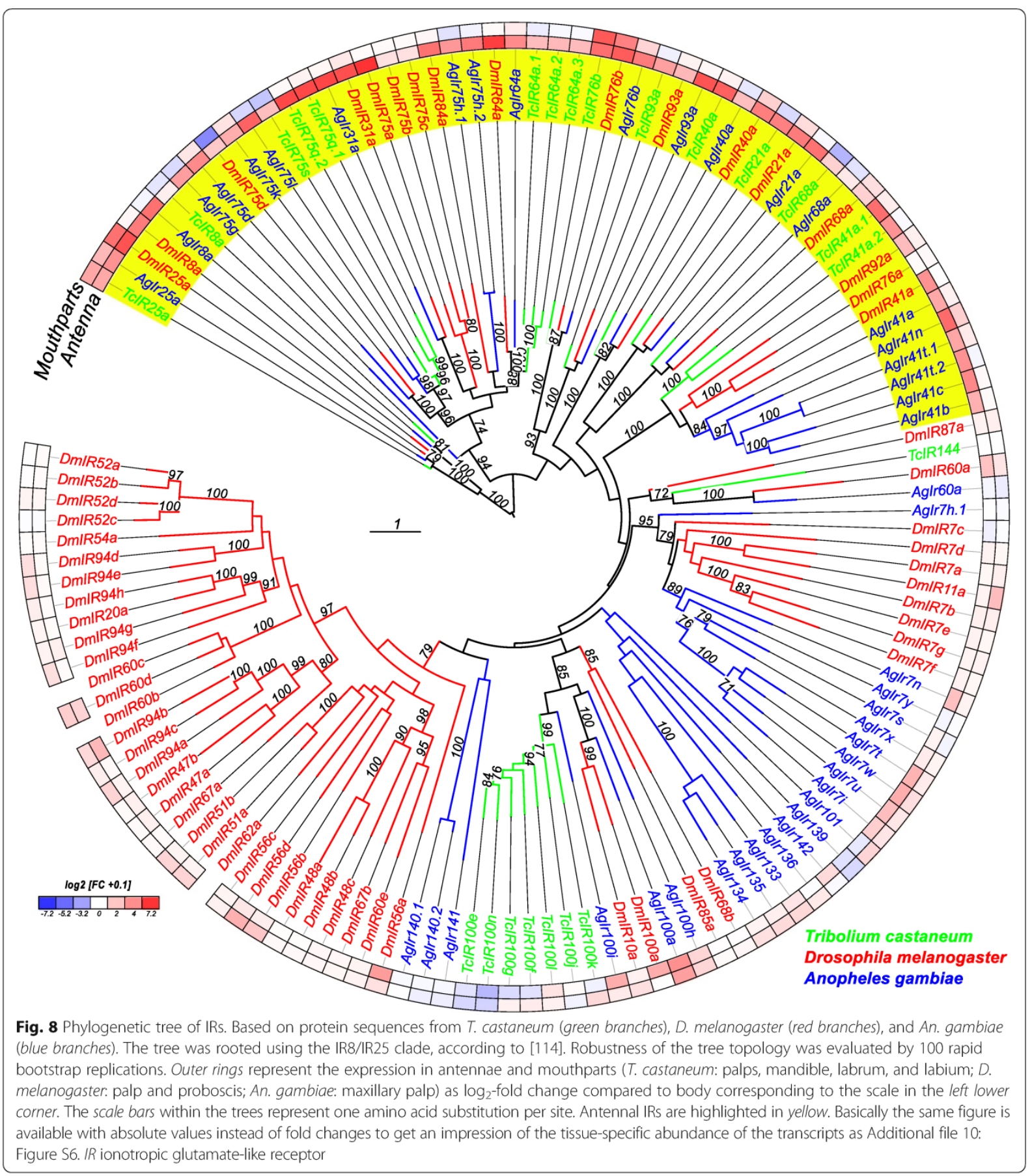

three also in the mouthparts (Fig. 13). One of these three, TcasGSTd2 represents a member of the GST delta subfamily such as GST-msolf1 from Manduca sexta, which is an olfactory-specific GST expressed specifically in the sexpheromone-detecting sensilla [149]. Analysis of the 141 previously described cytochrome P450s (CYPs) [150] revealed that two predicted gene models (CYP347A4 and
CYP351B1) were fusions of two separate genes (now termed CYP347A4A and CYP347A4B, as well as CYP351B1A and CYP351B1B, respectively). Seven other predictions had to be adjusted based on RNAseq data (sequences available in Additional file 8: Table S1). The expression analysis of these 141 genes showed that 26 are significantly enriched in the antenna, with 11 also in the 


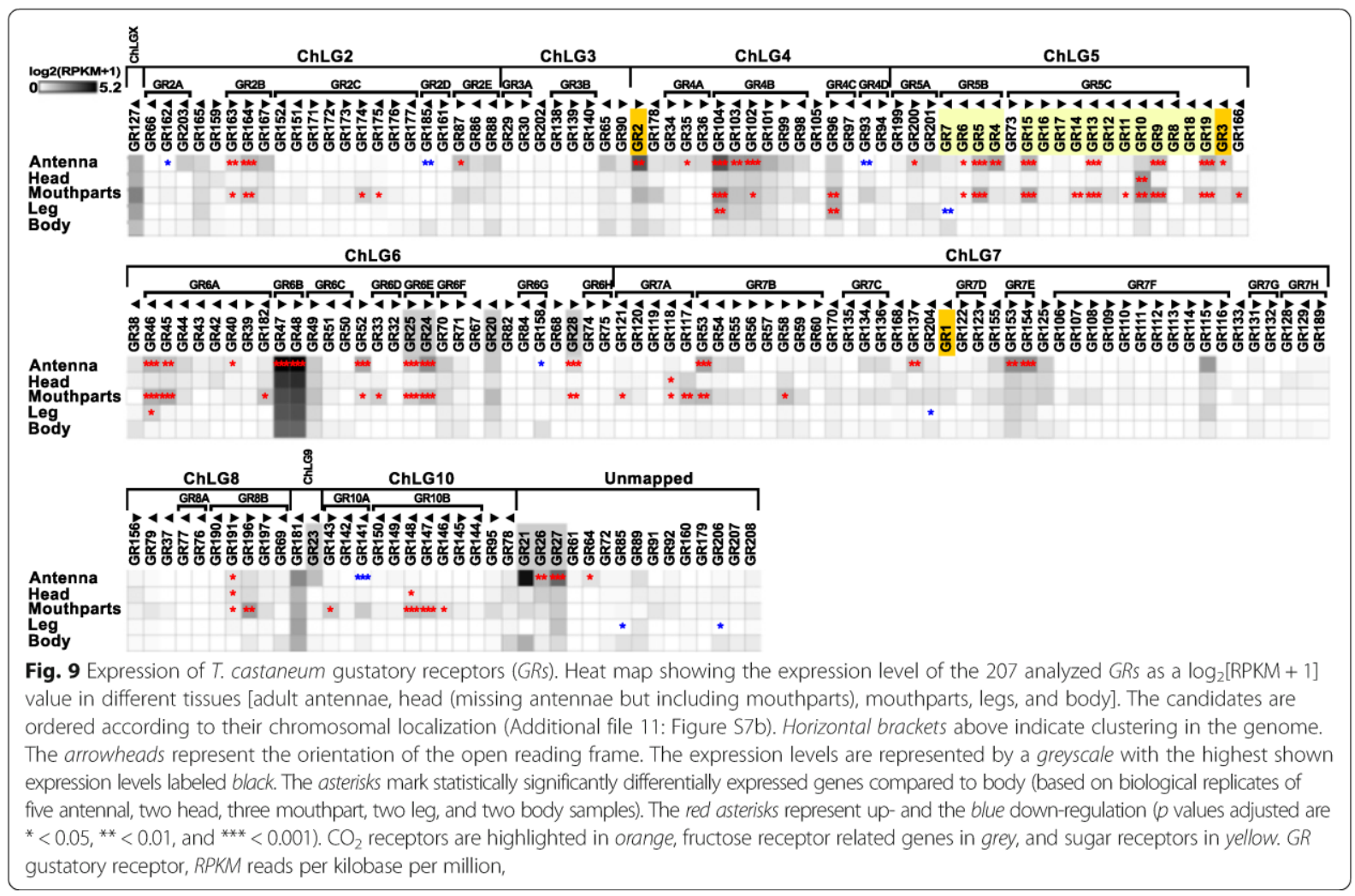

mouthparts (Fig. 13). In addition, six CYPs are significantly enriched in mouthparts, but not in antennae. For the coleopteran Phyllopertha diversa, CYPs have been shown to be involved in pheromone degradation in a membrane-bound manner [51].

\section{Expression of potential olfaction signal transduction pathway components}

The orthologs of genes encoding signal transduction pathway components known to be involved in olfaction of D. melanogaster [46] were identified by BLAST and manually curated. The expression analysis revealed that four of them $(r d g B, i t p r, d g k d$, and $d g k t)$ are significantly enriched in the antennae (Fig. 14). However, there is no chemosensory-specific candidate exclusively expressed in antennae or mouthparts. Our data, therefore, do not indicate a chemosensory-specific metabotropic signal transduction pathway.

\section{Expression and distribution of sensory neuron membrane proteins}

The transcriptome analysis revealed that one of the seven previously identified TcasSNMPs [116, 117], namely XP_969729 [116], was incorrectly annotated and does not encode for a CD36-related protein. Moreover, the gene model previously named SNMP1c (XM_001816389) was a fusion of two SNMPs and overlaps with SNMP1d (XM_001816391) [117]. In our re-annotation, we removed XP_969729 and separated TcasSNMP1c and TcasSNMP1d. In addition, the gene models of TcasSNMP2, TcasSNMP1a, and XP_975606 [116] had to be modified based on transcriptome and RACE-PCR data. For XP_975606, we propose the name TcasSNMP3, to reflect its unclear phylogenic relationship. Despite the more SNMP1-like expression pattern (Fig. 15) and chromosomal localization (Additional file 9: Figure S5b) of TcasSNMP3, the comparison of the amino acid composition revealed no clear affiliation to either the SNMP1 or the SNMP2 subgroup [151]. Interspecies comparison revealed no clear orthology of TcasSNMP3 to SNMPs from other species, including the so-called SNMP3 of Calliphora stygia [152], which, based on phylogeny, clearly represents an SNMP1 homolog. All six TcasSNMPs are expressed in antennae (Fig. 15), which was also confirmed by rapid amplification of cDNA-ends PCR (RACE-PCR) based on an antennae cDNA pool, but only TcasSNMP1a-d and TcasSNMP3 are significantly enriched in antennal tissue. Moreover, three of the TcasSNMP1, as well as TcasSNMP3, are also enriched in mouthparts (Fig. 15), further supporting the importance of the mouthparts for olfaction in T. castaneum. In contrast, 


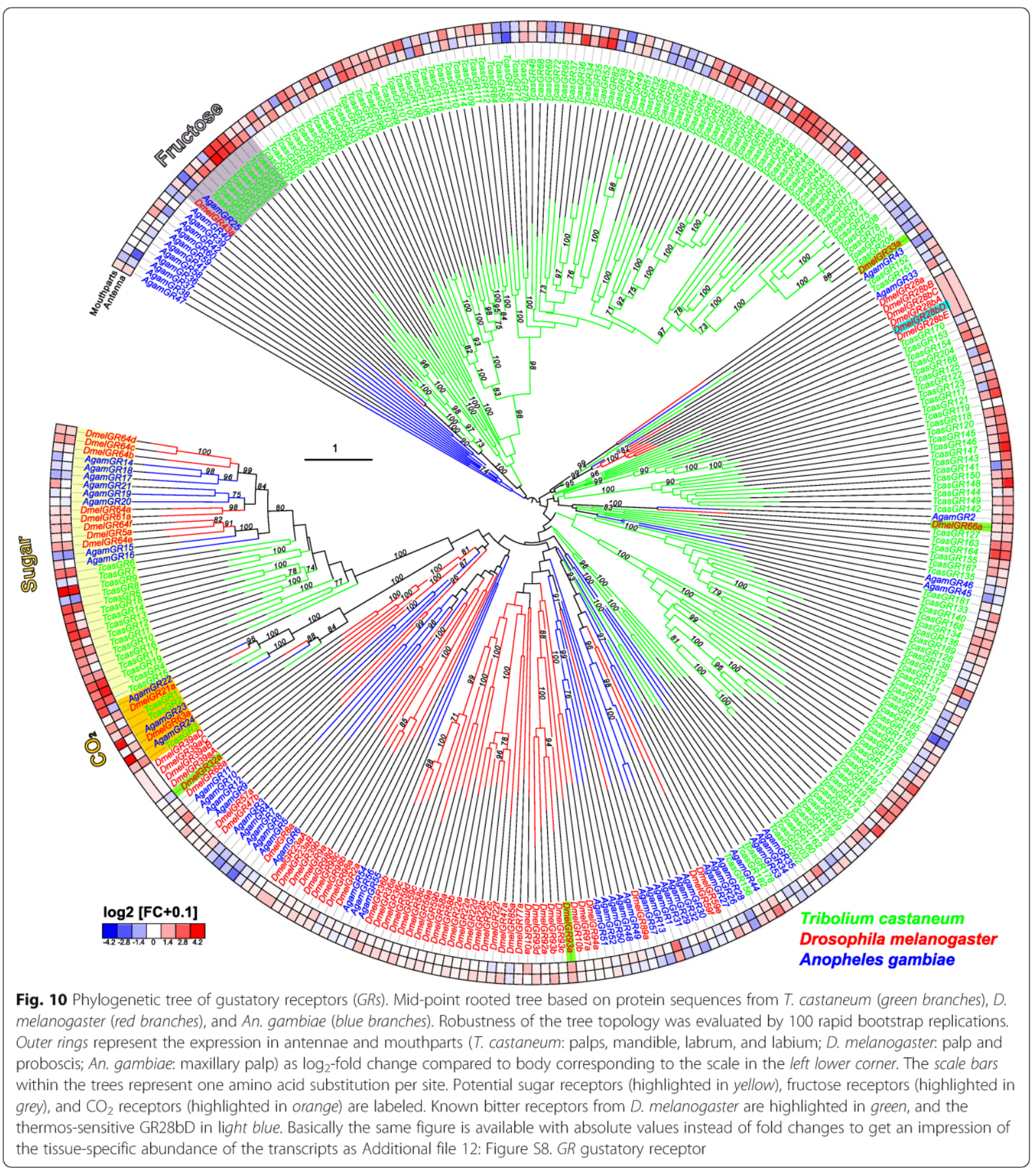

TcasSNMP2 is expressed highest in body and significantly underrepresented in antennae and mouthparts (Fig. 15), which is similar to its ortholog in D. melanogaster [49]. Despite the observation that in most insects with a fully sequenced genome only two SNMPs were found [116, 117], the relatively high amount of six TcasSNMPs of T. castaneum is not unique, since transcriptome analysis, e.g., of other beetles, revealed four SNMPs in Dendroctonus valens [153] and Dastarcus helophoroides [154], as well as three in Ips typographus and Dendroctonus ponderosae [136]. However, T. castaneum is currently only exceeded by the hessian fly (Mayetiola destructor) with seven expressed SNMPs [155]. 


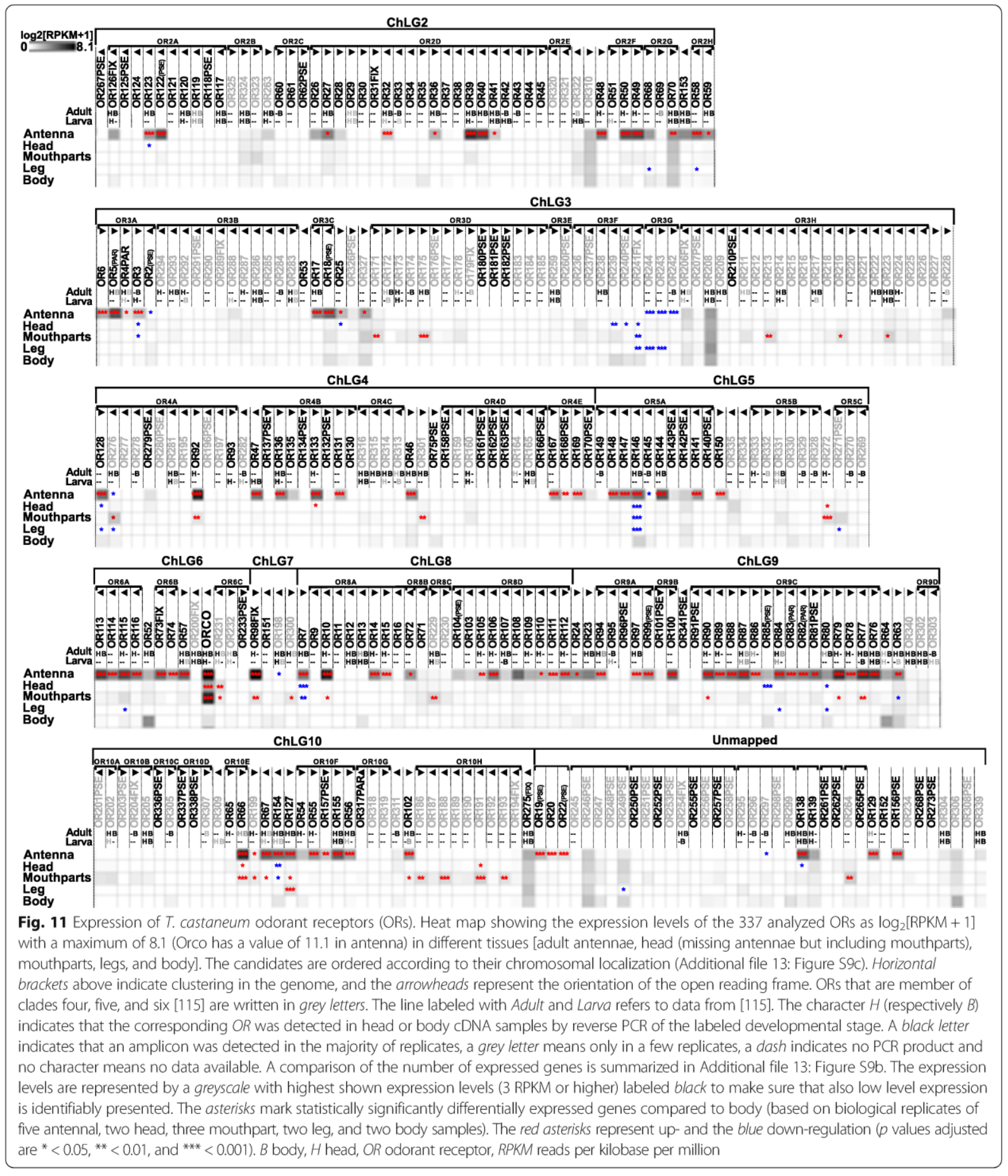

\section{Discussion}

Independent integration centers for antennal and palpal olfactory perception

In $T$. castaneum, odorants are mainly perceived with the last three segments of the antenna, which carries three types of chemoreceptive sensilla (SBas, cSTri, and SCoe), as well as with the maxillary and labial palps (Fig. 16). Accordingly, expression analysis revealed that ORs are mostly expressed in antennae, but also in the mouthparts (Figs. 11 and 12; Additional file 13: Figure S9a) as previously shown for several dipteran species [128, 129, 137, 156-158]. In contrast to the Diptera, where the 


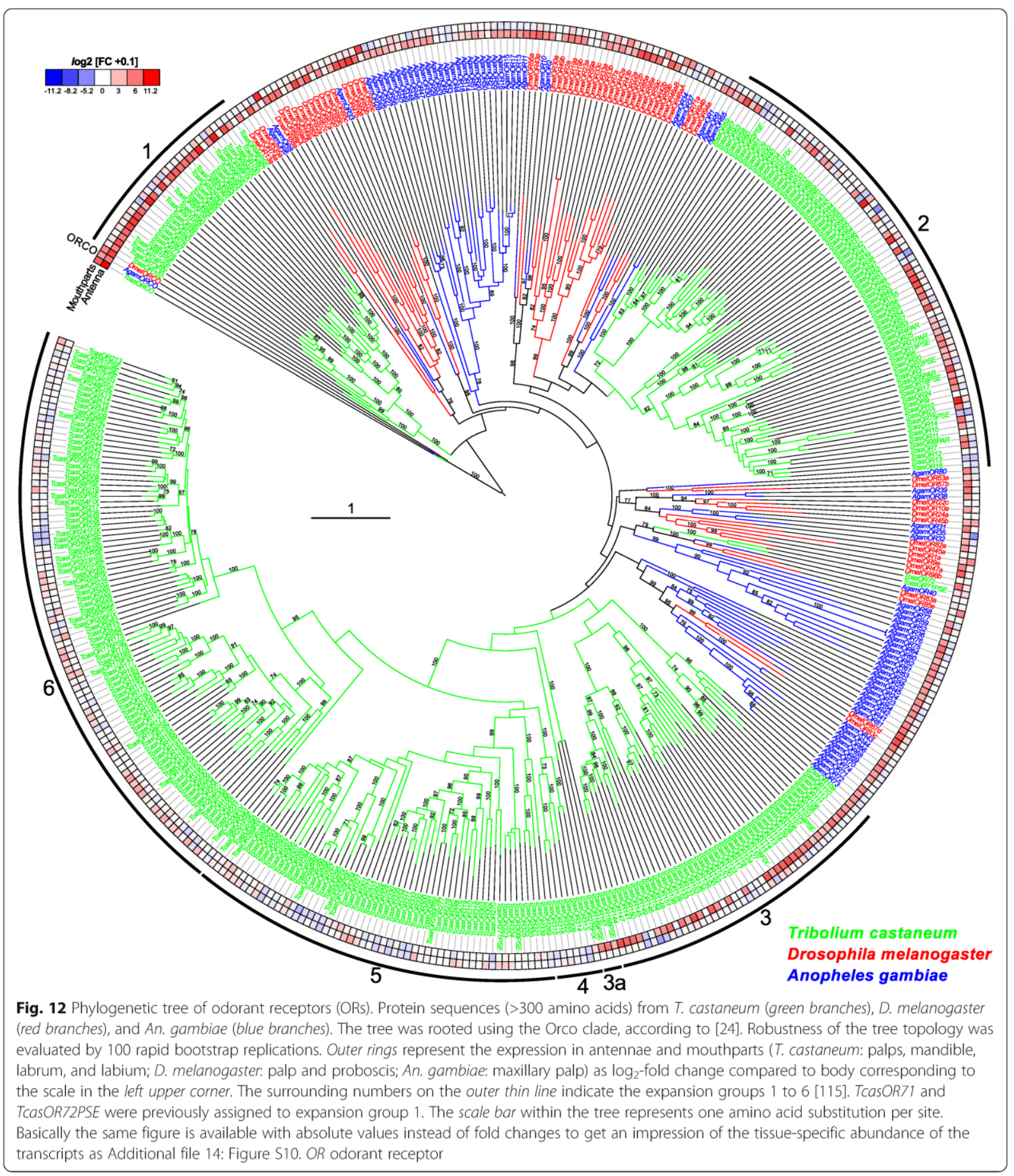

palps are chemosensory appendages with limited odor coding complexity, the relatively high number of Orcoimmunoreactive CSNs (Fig. 4) as well as the high number of expressed ORs, SNMPs, potential ODEs, and OBPs [89] in T. castaneum mouthparts (Figs. 11, 13, 15, and 16) imply a more prominent role of the palps in olfaction. The palpal ORs are possibly involved in the evaluation of the quality of food sources, like the ORs on the proboscis of Manduca sexta [159].

Moreover, in addition to the differences on the perception level, major dissimilarities to the Diptera occur on the level of odor processing. The data from the partial 


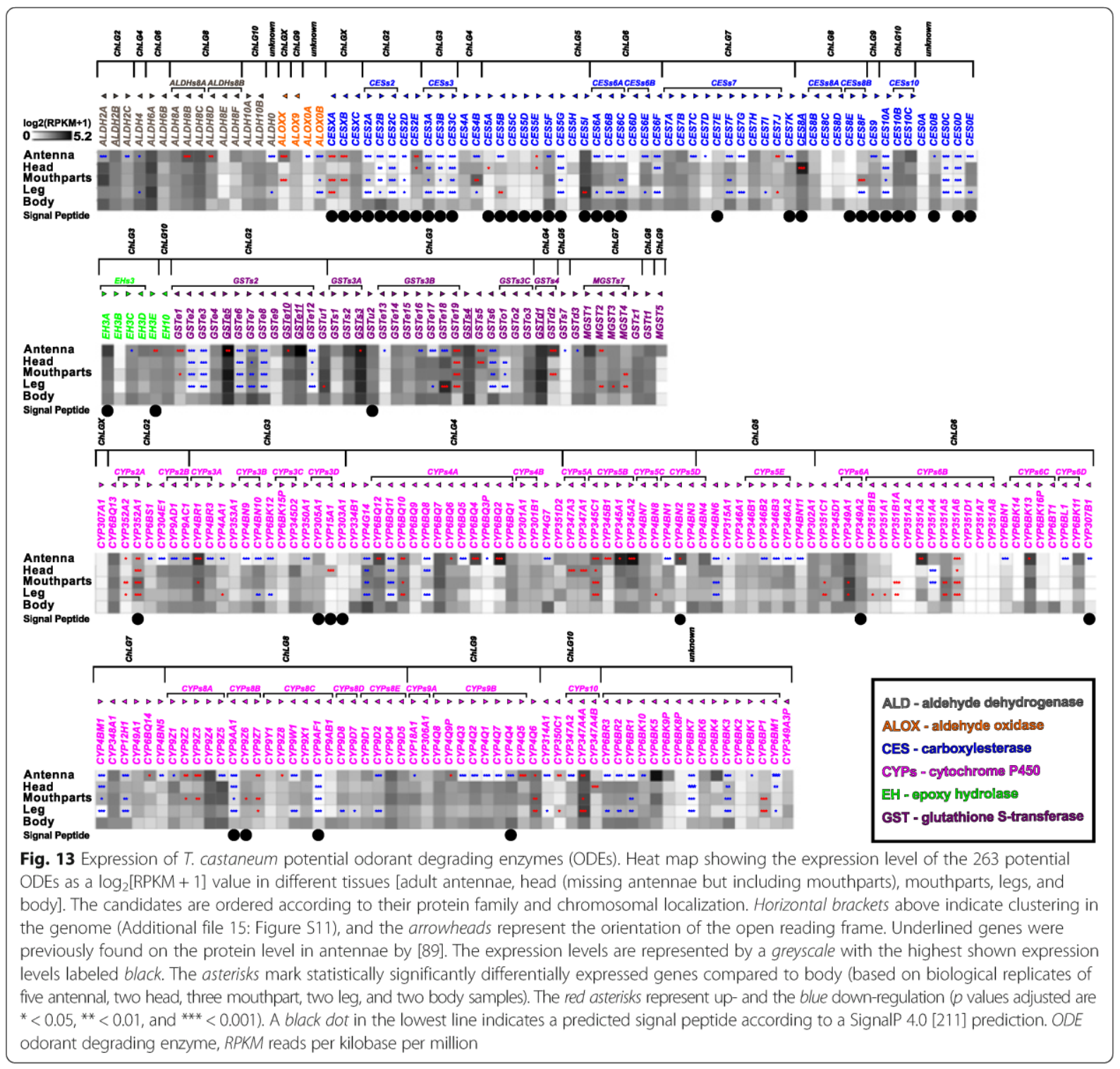

Orco-Gal4 line as well as the backfills from the antenna and the mouthparts indicate that processing olfactory information at least at the level of the first central relay station occurs independently of each other (Fig. 16). This is surprising, as many of the ORs expressed on the mouthparts are also expressed on the antennae. In contrast, typical OR expression is mutually exclusive between antenna and palps in D. melanogaster and An. gambiae [128, 137], where in addition, projections from the palps innervate several AL glomeruli $[63,160,161]$. In $T$. castaneum, the olfactory input stemming from the antenna seems to be processed exclusively in the AL (Fig. 3a; Additional file 5: Movie S2; Additional file 6:
Figure S4), whereas the palpal-derived olfactory information is essentially processed outside the AL, in the LG (Fig. 3e, f; Additional file 5: Movie S2) and the GOC, an unpaired and glomerularly organized first olfactory center in the GNG (Fig. 3d, f; Additional file 7: Movie S3). The LG had, as far as we know, previously been described only in hemimetabolous insects [57, 64-66, 105]. A glomerularly organized olfactory center in the GNG such as the GOC has, to our knowledge, not been described in any insect so far. The number of 49 ORs (with 28 being significantly enriched compared to body) that are expressed in the mouthparts is roughly consistent with the estimated 30 to 40 glomeruli in the GOC. This 


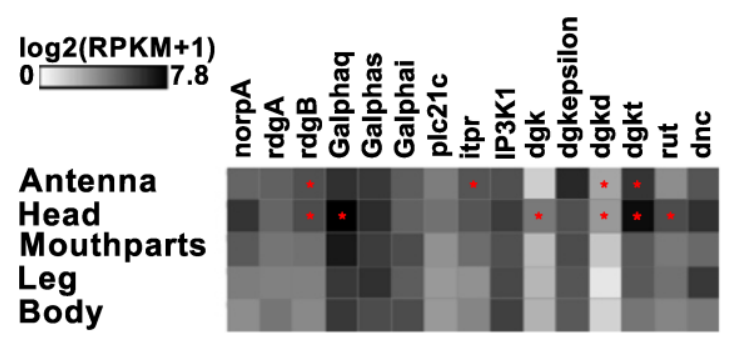

Fig. 14 Expression of T. castaneum homologs of genes described to be involved in olfaction of D. melanogaster. Heat map showing the expression level of the several genes supposed to be involved in D. melanogaster olfaction, as a $\log _{2}[R P K M+1]$ value in different tissues [adult antennae, head (missing antennae but including mouthparts), mouthparts, legs, and body]. The expression levels are represented by a greyscale with highest shown expression levels labeled black. The asterisks mark statistically significantly differentially expressed genes compared to body (based on biological replicates of five antennal, two head, three mouthpart, two leg, and two body samples). The red asterisks represent up-regulation ( $p$ values adjusted are $\left.{ }^{*}<0.05\right)$. RPKM reads per kilobase per million

suggests that the wiring in the GOC may resemble the situation in the ALs with the difference being convergence into an unpaired medial structure. The only palpal projection into the $\mathrm{AL}$ is a mutually exclusive innervation of a single ipsilateral glomerulus (Fig. 3a; Additional file 5), which may be involved in $\mathrm{CO}_{2}$ perception, as described in several moth species [99] and proposed for some mosquitoes [161, 162].
Antennae serve also as key organs for gustatory perception

In T. castaneum, antennae and mouthparts express similar high numbers and levels of GRs, which indicates the antenna as a key gustatory organ besides the mouthparts (Fig. 9; Additional file 11: Figure S7a). This finding may reflect the beetles' ground-dwelling life style and indicates that the scanning behavior with the antennae not

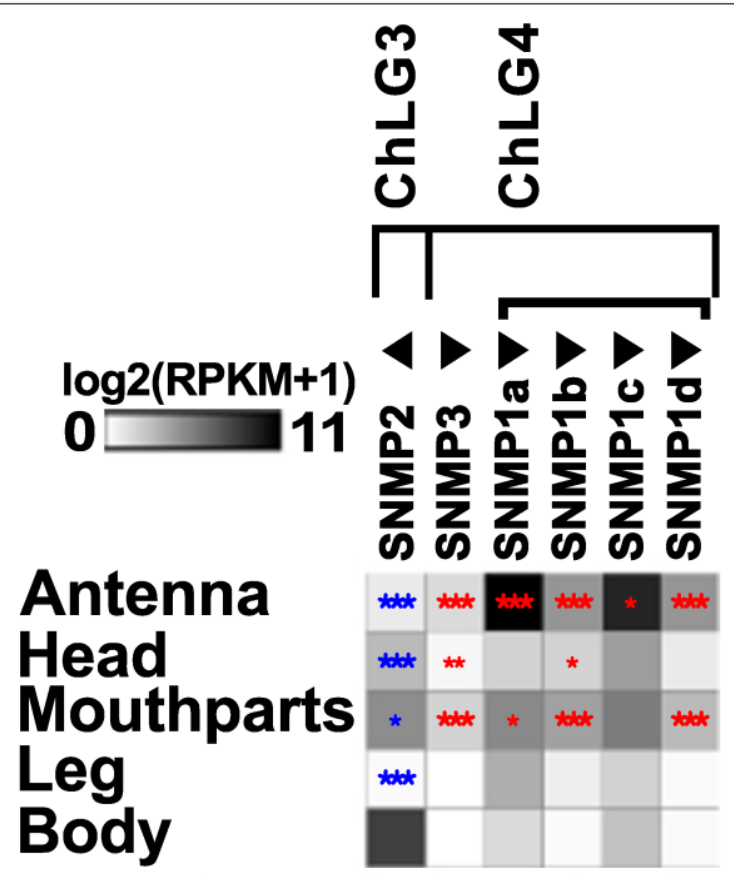

Fig. 15 Expression of T. castaneum sensory neuron membrane proteins (SNMPs). Heat map showing the expression level of the six sensory neuron membrane proteins of $T$. castaneum, as a $\log _{2}[R P K M+1]$ value in different tissues [adult antennae, head (missing antennae but including mouthparts), mouthparts, legs, body, as well as larval head and body. The candidates are ordered according to their chromosomal localization (Additional file 9: Figure S5b). Horizontal brackets above indicate clustering in the genome, and the arrowheads represent the orientation of the open reading frame. The expression levels are represented by a greyscale with highest shown expression levels labeled black. The asterisks mark statistically significantly differentially expressed genes compared to body (based on biological replicates of five antennal, two head, three mouthpart, two leg, and two body samples). The red asterisks represent up- and the blue down-regulation ( $p$ values adjusted are ${ }^{*}<0.05,{ }^{*}<0.01$, and ${ }^{* * *}<0.001$ ). SNMP sensory neuron membrane protein, RPKM reads per kilobase per million 


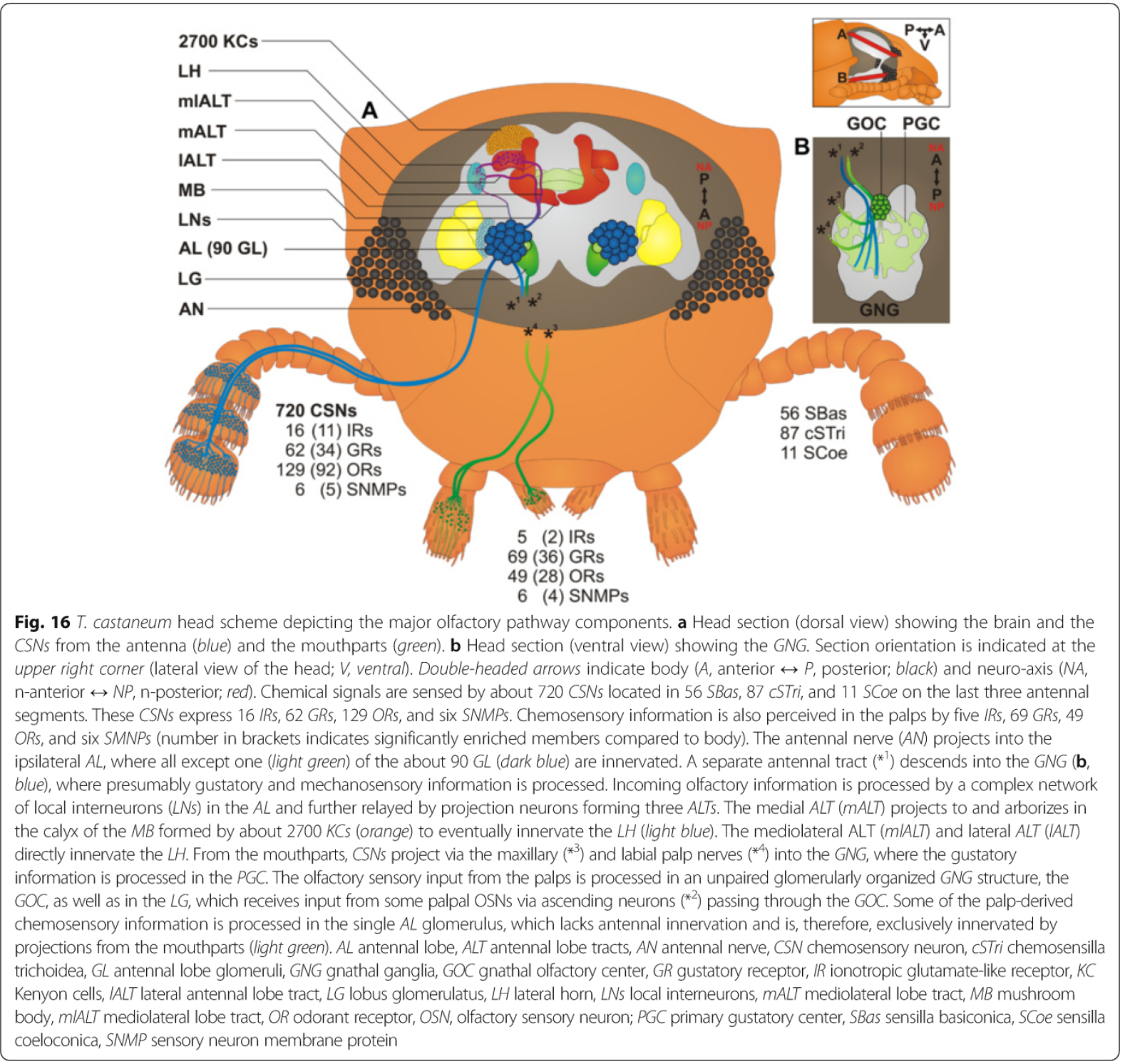

only gathers tactile but also chemical stimuli. This is in contrast to the Diptera, where the labellum is the main gustatory organ [163-165].

\section{Postulation of exceptions to the central dogma}

The number of 129 ORs that we found to be expressed in T. castaneum antennae (Fig. 11; Additional file 13: Figure S9a) exceed the numbere of about 90 glomeruli in the AL. Moreover, some glomeruli are likely to get exclusive innervation by OSNs that express IRs, as described in D. melanogaster [20]. These observations do not conciliate with the central dogma postulating that OSNs express only one typical OR and all OSNs carrying this same OR converge into one and the same glomerulus, which was hypothesized to be the typical situation for insects $[61,137,166]$. However, for D. melanogaster, both co-expression of more than one typical OR per OSN as well as co-convergence due to innervation of one AL glomerulus by more than one OSN sub-type have been already described as exceptions [137, 167]. For T. castaneum, we propose that such exceptions are much more frequent.

\section{Large repertoire of potentially functional odorant receptor genes and possible environmental regulation}

The genome of $T$. castaneum harbors 341 OR genes [81, 115], of which 270 seem to encode for functional ORs. Of the 337 ORs with available full sequence information [115], we find in our RNAseq data 161 ORs to be expressed in adult antennae, mouthparts, and head by a threshold of 0.5 RPKM (Additional file 13: Figure S9a). 
In comparison to the RT-PCR-based data from [115], who found 112 ORs to be clearly expressed in adult heads, we only confirmed 82 ORs. In addition, we identified 41 ORs previously declared as not expressed and 37 ORs previously not tested [115] as expressed (Additional file 13: Figure S9b). This discrepancy may partially be due to the different type of methodology used to identify expression. However, culturing conditions and the specific genetic variations of the strain used may also be responsible for the differences.

Taking both studies together, there is clear experimental evidence for 191 ORs that are expressed in the adult head. By including adult leg and all adult body data, 223 ORs seem to be expressed in total, of which 17 actually do not encode an intact OR. However, for 64 OR functional gene models, no expression could be detected so far. This may be due to low expression in a single OSN or conditional expression under exceptional circumstances. The red flour beetle can live for up to two years [168]. During this long period in their natural environment, the beetles can encounter a variety of challenges such as food shortages, which possibly triggers flight migrations over tens of kilometers [169]. Under such exceptional circumstances, the not or low-expressed receptor genes may become active [115], as shown in studies in D. melanogaster [170] and An. gambiae [171] where up to fivefold upregulation of several ORs was triggered by temperature or feeding state.

\section{Inter-species comparison of olfactory components}

The comparison of the number of main components of the chemosensory pathway of different insect species reveals the high diversity of evolutionary strategies to enable proper chemoreception and thus, reflects the diversity of insects and the manifold adaptations to their specialized lifestyles (Table 1). In particular, T. castaneum has by far the lowest number of chemoreceptive sensilla (154) and consequently also of CSNs (720). In contrast to this low number, the number of GRs (220) and ORs (341), but not of the IRs (23) encoded in the genome is exceptionally high. The number of olfactory glomeruli in the $\mathrm{AL}$ is within the range of most other species (Table 1) $[57,172]$. Comparing the relation of OR genes and number of glomeruli, the highest discrepancy occurs with about fourfold higher numbers of OR genes in T. castaneum. However, also in Aedes aegypti, OR gene numbers are more than double the number of glomeruli [165]. In most other analyzed insects, except ensiferan orthopterans that have hundreds of microglomeruli [173, 174], the number of OR genes is typically similar to the number of glomeruli (Table 1). Despite the relatively low number of IRs encoded in the genome of $T$. castaneum, the repertoire of IRs involved in olfaction is highly conserved (Fig. 8). The number of KCs is roughly the same as in $D$. melanogaster and seems to be independent of the OR or AL glomeruli number (Table 1) [175].

\section{No apparent sexual dimorphism}

Sexual dimorphism of the olfactory system is described in many insect species [57, 172, 176, 177]. However, in contrast to other coleopterans [178-182], our analysis revealed no apparent sexual dimorphism on antenna morphology or number and distribution of sensilla (Additional file 1: Figure S1b-d). Expression analysis of male and female antenna samples revealed only a small but not significant dimorphism in the OBP expression levels described earlier [89]. Also for IRs, GRs, ORs, and SNMPs, we could not find any significant sexual differences (Fig. 6), similar to the striped flea beetle Phyllotreta striolata [183] and in contrast to described situations in Diptera and Lepidoptera [63, 128, 184, 185]. Different numbers of glomeruli or different sized glomeruli were observed in several insect species [57, 70, 176] including the beetle Holotrichia diomphalia [172]. However, the comparison of the ALs of T. castaneum males and females disclosed no obvious dimorphism as previously described also for the small hive beetle (Aethina tumida) [186]. In summary, our study did not reveal any sexual dimorphism of the olfactory system in T. castaneum. This finding is consistent with behavioral studies that showed an attraction of both sexes to the aggregation pheromone 4,8-dimethyldecanal [187] and no sex preference in the mating choice of males [188].

\section{Conclusions}

Detailed analysis of the olfactory system in T. castaneum, a holometabolous insect of special importance for the study of coleopteran and pest biology, reveals that olfactory sensory input from the antennae is processed mostly in the antennal lobes of the brain, as observed in other insect species. However, tracing of olfactory projections from the mouthparts enabled the identification of two additional neuropils: a lobus glomerulatus described previously only in a hemimetabolous insect and an unpaired glomerularly organized olfactory neuropil in the GNG (the GOC), which has never before been described. In addition, the high number of GRs on both the antennae and mouthparts indicates that there is no organotopic separation of olfaction and gustation in this beetle. These findings are a reminder of the wide variety of solutions to chemoreception that have evolved in the holometabolous insects. This should remind us that we have much still to learn about olfactory systems in general.

\section{Methods}

Tribolium castaneum rearing and transgenic lines

Tribolium castaneum (Herbst, 1797; Insecta, Coleoptera, Tenebrionidae) wild-type strain San Bernardino, as well 
Table 1 Comparison of main components of the olfactory system of different insect model organisms

\begin{tabular}{|c|c|c|c|c|c|c|c|}
\hline Species & $\begin{array}{l}\text { Chemo-receptive sensilla } \\
\text { (per antenna) }\end{array}$ & $\begin{array}{l}\text { CSNs } \\
\text { (per antenna) }\end{array}$ & $\begin{array}{l}\text { IRs } \\
\text { (genes) }\end{array}$ & $\begin{array}{l}\text { GRs } \\
\text { (genes) }\end{array}$ & $\begin{array}{l}\text { ORs } \\
\text { (genes) }\end{array}$ & $\begin{array}{l}\text { AL glomeruli } \\
\text { (per } \mathrm{AL} \text { ) }\end{array}$ & KCs (per MB) \\
\hline T. castaneum & 154 & 720 & $23^{1}$ & $220^{2}$ & $341^{3}$ & $70^{4}-90$ & 2700 \\
\hline D. melanogaster & $530^{5}$ & $1200^{5,6}$ & $66^{1}$ & $73^{7}$ & $62^{8}$ & $43^{6}-54^{9}$ & $2500^{10}$ \\
\hline \multirow[t]{2}{*}{ An. gambiae } & $714 f^{11 a}$ & $1500-1600 f^{11 a}$ & $46^{1}$ & $60^{7}$ & $79^{8}$ & $60 f^{14}$ & $\mathrm{n} / \mathrm{a}$ \\
\hline & $738 f^{12}$ & & & & $76^{13}$ & $61 m^{14}$ & \\
\hline \multirow[t]{2}{*}{ Ae. aegypti } & $928 f^{15}$ & $1946 f^{15}$ & $95^{1}$ & $79^{16}$ & $131^{17}$ & $50 f^{18}$ & $\mathrm{n} / \mathrm{a}$ \\
\hline & & & & & & $49 m^{18}$ & \\
\hline \multirow[t]{2}{*}{ Bombyx mori } & $>24500 \mathrm{~m}^{19 a}$ & $50000 \mathrm{~m}^{19 a}$ & $18^{1}$ & $56^{7}$ & $48^{7}$ & $55-60^{19}$ & $\mathrm{n} / \mathrm{a}$ \\
\hline & $>21000 f^{19 a}$ & $30000 f^{19 a}$ & & & & & \\
\hline \multirow[t]{3}{*}{ M. sexta } & $190000 \mathrm{~m}^{20}$ & $255000-$ & $21^{22}$ & $45^{22}$ & $71^{22}$ & $63^{23}$ & $\mathrm{n} / \mathrm{a}$ \\
\hline & & $450000 \mathrm{~m}^{21 \mathrm{a}, 20}$ & & & & & \\
\hline & & $169000 f^{21 a}$ & & & & & \\
\hline \multirow[t]{2}{*}{ A. mellifera (worker) } & $5000-5100^{24}$ & $60000^{25 a}$ & $10^{1}$ & $53^{7}$ & $163^{8}$ & $156-166^{26}$ & $170000^{27}-184000^{28}$ \\
\hline & & $63700^{24}$ & & & & & \\
\hline \multirow[t]{2}{*}{ Periplaneta americana } & $65500 \mathrm{~m}^{29}$ & $241000 \mathrm{~m}^{29}$ & $\mathrm{n} / \mathrm{a}$ & $\mathrm{n} / \mathrm{a}$ & $\mathrm{n} / \mathrm{a}$ & $125 f^{29}$ & $175000^{30}$ \\
\hline & & & & & & $126 \mathrm{~m}^{29}$ & \\
\hline \multirow[t]{2}{*}{ Locusts } & Lmig $4700^{31}$ & $50000^{32 a}$ & $\operatorname{Lmig} 11^{7}$ & $\operatorname{Lmig} 75^{7}$ & $\operatorname{Lmig} 95^{7}$ & Sgre $1000^{32}$ & Sgre $50000^{32}$ \\
\hline & & & & & & Sgre $2500-3000^{33}$ & \\
\hline
\end{tabular}

AL antennal lobe, CSN chemosensory neuron, $f$ female, GR gustatory receptor, IR ionotropic glutamate-like receptor, KC Kenyon cells, $L$ mig Locusta migratoria, $m$ male, $n / a$ not available, $O R$ odorant receptors, Sgre Schistocerca gregaria

alfactory sensory neurons/sensilla (otherwise chemosensory neurons/sensilla).

${ }^{1}$ Croset et al. 2010 [114], ${ }^{2}$ Richards et al. 2008 [81], ${ }^{3}$ Engsontia et al. 2008 [115], ${ }^{4}$ Dreyer 2010 [200], ${ }^{5}$ Stocker 2001 [59], ${ }^{6}$ Vosshall and Stocker 2007 [137], ${ }^{7}$ Wang et al. 2014b [223], ${ }^{8}$ Sánchez-Gracia et al. 2001 [25], ${ }^{9}$ Grabe et al. 2015 [224], ${ }^{10}$ Hinke 1961 [225], ${ }^{11}$ Qiu et al. 2006 [226], ${ }^{12}$ Pitts and Zwiebel 2006 [227], ${ }^{13}$ Pitts et al. 2011 [128], ${ }^{14}$ Ghaninia et al. 2007 [160], ${ }^{15}$ Mclver 1978 [228], ${ }^{16}$ Kent et al 2008 [229], ${ }^{17}$ Bohbot et al. 2007 [165], ${ }^{18}$ Ignell et al. 2005 [162], ${ }^{19}$ Koontz and Schneider 1987 [230], ${ }^{20}$ Lee and Strausfeld 1990 [231], ${ }^{21}$ Homberg et al. 1989 [232], ${ }^{22}$ Kanost et al. 2016 [233], ${ }^{23}$ Rospars and Hildebrand 2000 [234], ${ }^{24}$ Esslen and Kaissling 1976 [235], ${ }^{25}$ Frasnelli et al. 2010 [236], ${ }^{26}$ Galizia et al. 1999 [237], ${ }^{27}$ Mobbs 1982 [238], ${ }^{28}$ Strausfeld 2002 [239], ${ }^{29}$ Boeckh and Ernst 1987 [240], ${ }^{30}$ Neder 1957 [241], ${ }^{31}$ Greenwood and Chapman 1984 [242], ${ }^{2}$ Laurent and Naraghi 1994 [243], ${ }^{33}$ Schachtner et al. 2005 [57]

as the transgenic lines partial Orco-Gal4, UAS-DsRed, UAS-tGFP [87], and EF1-B-DsRed [189] were bred at about $30{ }^{\circ} \mathrm{C}$ and $40 \%$ relative humidity on organic whole wheat flour supplemented with $5 \%$ yeast powder [190]. The Orco-Gal4 and UAS-DsRed lines were generated by piggyBac-based insertional mutagenesis [191]. The donor plasmids used were assembled by a versatile two-step cloning procedure [192].

For the partial Orco-Gal4 line, a donor plasmid was generated by cloning a blunted and BamHI (Fermentas, Vilnius, Lithuania) digested $\mathrm{PCR}<$ product containing Gal4delta-SV4OpA (amplified with primers Gal4deltafor and SV40rev from plasmid $\mathrm{CH} \# 757$, see Additional file 16) into the BamHI and EcoRV (Fermentas) digested pSLfa1180 vector [193]. After propagation, a BamHI and BfuAI digested PCR product containing $2.5 \mathrm{~kb}$ upstream of the TcasOrco (amplified with TcOR1upfor and TcOR1uprev from San Bernardino gDNA) was cloned into the corresponding restriction sites to generate pSLfa $1180[2.5 \mathrm{kbOr}-$ coUp_GAL4delta]. The whole cassette was shuttled with AscI and FseI (New England Biolabs, Ipswich, MA, USA) into the pBac[3XP3-Tcv] [194] donor plasmid. The tissuespecific expression of Gal4 in the Orco-Gal4 line was determined by crossing it with an UAS-tGFP [87] line and performing IHC on the antennae with $\alpha$-tGFP and $\alpha$-Orco antibody or by staining of the whole brain with $\alpha$-tGFP and an $\alpha$-synapsin counterstaining. These stainings revealed that only Orco-immunoreactive neurons are labeled in antennae (Additional file 2: Figure S2d), which indicates the specificity of the Orco-Gal4 driver line. However, only half of the Orco-immunoreactive neurons in the antenna express tGFP (Additional file 2: Figure S2d), which implies that the Orco-Gal4 line only partially covers the Orco pattern resulting in labelling of only half of the AL glomeruli (Additional file 2: Figure S2e). The same approach with an $U A S$-dsRed line and an $\alpha$-RFP antibody was used to characterize the palps, in which the reporter is also exclusively expressed in Orco-immunoreactive neurons, but in only $10-20 \%$ of the cells (Additional file 2: Figure S2f). We, therefore, refer to it as the partial Orco-Gal4 line.

For $U A S$-DsRed, the donor plasmid pBac[3XP3-eYFP_UAS-Tchsp68bP-DsRedex-SV40] was generated by cloning the DsRed express ORF (Clontech laboratories Inc., Mountain View, CA, USA; catalog no. 632412) into the pSLfa[UAS-Tc'Hsp-p-tGFP-SV40]fa shuttle vector [87] by using KpnI and NotI, which was followed by transferring 
the UAS-hsp-DsRed-SV40 cassette into the pBac[3XP3eYFP] [193] using AscI and FseI. The UAS-DsRed line as well as the UAS-tGFP line were analyzed by confocal microscopy to ensure that no reporter expression was present in the relevant tissues in the absence of a Gal4 driver line (Additional file 17: Figure S12).

The EF1-B-DsRed line (elongation factor1-alpha regulatory region-DsRedExpress; kindly provided by Michalis Averof, Institut de Génomique Fonctionnelle de Lyon, France) has been described to label most neurons in the central nervous system of first instar larvae [189] and also shows high expression in the adult central nervous system. However, clearly not all neurons are labeled in the peripheral nervous system. We, therefore, reanalyzed adult antennae of this line using confocal microscopy in combination with antibody stainings. The labeled neurons in the antenna resemble the typical morphology of CSNs with the dendrites being embedded in the sensilla cavities (Fig. 2h; Fig. 1f, g, and h) and the axons converging to the antennal nerve (Fig. 2h; Additional file 1: Figure S1a). No labelling was detected at mechanosensory sensilla (Fig. 1c, d, and e) except the scolopidia cells of Johnston's organ (Additional file 1: Figure S1a). In addition to almost all Orco-immunoreactive ORNs (Fig. 2i), this line labels also non Orcoimmunoreactive neurons that are affiliated with sensilla coeloconica (Fig. 1h) and sensilla basiconica (Fig. 1g). Whereas in the palps only about 30 to $50 \%$ of the DsRedimmunoreactive cells are also Orco-immunoreactive (Fig. 4), in antennal segment 11 a higher percentage of CSNs is double labeled, and in segments 9 and 10, the vast majority of CSNs are double labeled (Fig. 2i-i"; Additional file 1: Figure S1a). This suggests that almost all and only CSNs are labeled by this line in the adult antenna.

\section{Tissue preparation for SEM}

Antennae of sex-separated adults were dissected and immediately fixed for at least $2 \mathrm{~h}$ in $5 \%$ glutaraldehyde in $0.1 \mathrm{M}$ phosphate buffered saline (PBS), $\mathrm{pH} 7.1$, washed and post-fixed in osmium-tetroxide $(1 \%$ in $0.1 \mathrm{M}$ Sörensen buffer, pH 7.2). Fixed samples were washed in water, dehydrated overnight in ethyleneglycolmonoethylether, and then transferred into acetone via at least three 10-min changes with $100 \%$ acetone as described in [195]. The samples were critical-point-dried by using a Polaron E 3000 (Balzers Union, Quorum Technologies Ltd, Darmstadt, Germany). After being sputtered with gold (Balzers Union Sputter Coater, Balzers, Liechtenstein; Quorum Technologies Ltd, Ringmer, UK), the material was examined using a Hitachi S-530 SEM (Hitachi High-Technologies Europe GmbH, Krefeld, Germany). Micrographs (Figs. 1b-b", c"', c"'", d"', d"', e"-e'"', f", g"', g'"', h", and $2 \mathrm{a}-\mathrm{g}$ ) were taken by digital image acquisition (DISS 5, point electronic, Halle, Germany).

\section{Immunohistochemistry}

Whole mount brain IHC was performed as described in [102]. The animals were cold anesthetized, their brains were dissected in cold PBS (0.01 M, pH 7.4), and fixed subsequently overnight at $4{ }^{\circ} \mathrm{C}$ or for $1-2 \mathrm{~h}$ at room temperature in PBS containing $4 \%$ paraformaldehyde (Roth, Karlsruhe, Germany). The tissue was rinsed four times for 10 min with PBS. and pre-incubated with $5 \%$ normal goat serum (NGS, Jackson ImmunoResearch, Westgrove, PA, USA) in PBT (PBS containing $0.3 \%$ Triton X-100; Sigma-Aldrich, Steinheim, Germany) for 1-3 days at $4{ }^{\circ} \mathrm{C}$. After pre-incubation, nervous tissue was transferred to the primary antibody solution containing $2 \%$ NGS in PBT and incubated for 2-4 days at $4{ }^{\circ} \mathrm{C}$. To label neuropil regions selectively, a monoclonal primary antibody from mouse against synapsin was used in combination with specific additional antibodies and various dyes (for an overview of the antibodies and dyes employed, see Additional file 18: Table S2). After rinsing five times for $10 \mathrm{~min}$ with $\mathrm{PBT}$, the brains were incubated with appropriate secondary antibodies and various dyes (Additional file 18: Table S2) diluted in PBT containing $2 \%$ NGS for $1-3$ days at $4{ }^{\circ} \mathrm{C}$, followed by 3 to 5 washing steps for $10 \mathrm{~min}$ each with PBT. Brains and ganglia were dehydrated in an ascending ethanol series (50\%, $70 \%, 90 \%, 95 \%, 100 \%$, and $100 \%$ for $2.5 \mathrm{~min}$ each) and cleared with methyl salicylate (Merck, Gernsheim, Germany). Finally, they were mounted on coverslips using Permount mounting medium (Fisher Scientific, Pittsburgh, PA, USA) and a stack of two reinforcement rings (Zweckform, Oberlaindern, Germany) as spacers to prevent compression. Brains and ganglia of some of the backfills were not dehydrated and directly mounted in Aqua-Poly/ Mount (Polysciences Europe Inc., Eppelheim, Germany).

Antennae and palps of the EF1-B-DsRed, the OrcoGal4/UAS-tGFP, or Orco-Gal4/UAS-dsRed lines were dissected and fixed overnight at $4{ }^{\circ} \mathrm{C}$ in $4 \%$ paraformaldehyde and $10 \%$ methanol in PBT. Afterwards, they were transferred into silicone molds, embedded in tissue-freezing media (Leica, Wetzlar, Germany), and frozen for at least 1 hour at $-80{ }^{\circ} \mathrm{C}$, followed by cutting into $50 \mu \mathrm{m}$ sections at $-23{ }^{\circ} \mathrm{C}$ on a Cryotome (Cryotome CM 1959, Leica Microsystems, Wetzlar, Germany) resulting in longitudinally halved antennae. The half mounts were collected in a tube and rinsed four times for $20 \mathrm{~min}$ each at room temperature in PBT. The samples were pre-incubated with $5 \%$ NGS in PBT overnight at $4{ }^{\circ} \mathrm{C}$ followed by incubation with primary antibodies and dyes together with $5 \%$ NGS in PBT overnight. After washing four times for 20 min with PBT, the samples were incubated with appropriate secondary antibodies (Additional file 18: Table S2) overnight at $4{ }^{\circ} \mathrm{C}$. Finally, the antennae were rinsed four times with PBT for $20 \mathrm{~min}$ and 
embedded on coverslips in Aqua-Poly/Mount with one layer of reinforcement rings as spacers.

The specificity of the Orco-antiserum (Moth-R2, kindly provide by Jürgen Krieger) in T. castaneum could be demonstrated by IHC on antennae of animals with RNA interference-mediated knock-down of Orco [115]. To circumvent problems during dsRNA synthesis previously observed with the full length CDS of TcasOrco, we cloned a 476 bp fragment from San Bernadino cDNA containing only a part of CDS and the majority of the 3 ' untranslated region amplified by Advantage 2 Taq Polymerase and primers TcasOrco3UTRrev and TcasOrco3for (see Additional file 16) into PCRII vector (Invitrogen). Using PCR, a bidirectional template was generated followed by dsRNA synthesis with the MEGAscript T7 transcription kit (Ambion, Austin, USA) [196]. Orco dsRNA was injected into pupa of the strain San Bernardino. About 7 days after adult eclosion, the antennae of the treated animals were collected together with antennae of untreated beetles of the black strain, which can be easily discriminated based on the cuticle color, and thus, they served as internal staining controls. A maximal projection of a confocal stack of the Orco-antiserum (Moth-R2) treated antennae shows no detectable antibody staining in RNAi-treated animals (Additional file 2: Figure $\mathrm{S} 2 \mathrm{~b})$ in contrast to the black beetle internal control (Additional file 2: Figure S2c).

\section{In vivo backfills of the antenna, single maxillary palps,} and whole mouthparts

Cold anesthetized animals were mounted with dental wax (S-U-wax wire, $2.0 \mathrm{~mm}$, hard; Schuler Dental, Ulm, Germany) and modelling clay (Das grosse Dino-Knet-Set; Moses, Verlag GMBH, Kempen, Germany) using a lowtemperature soldering iron (Solder-Unit ST 081; Star Tec Products, Bremen, Germany) or with rubber cement (Fixogum, Marabu, Tamm, Germany) with their dorsal side on a microscope slide. The last three segments of the antenna and the most distal segment of the maxillary palp were removed and $4 \%$ neurobiotin in $1 \mathrm{M} \mathrm{KCl}$ (Vector Laboratories, Burlingame, UK) for the antenna and Texas Red coupled dextran $50 \mathrm{mg} / \mathrm{ml}$ in PBS (3000 MW; Molecular Probes, Invitrogen) for the maxillary palps were used as neuronal tracers. Glass micropipettes were drawn (Model P-97, Sutter Instrument, Novato, USA) from borosilicate glass (inner diameter, $0.75 \mathrm{~mm}$; outer diameter, $1.5 \mathrm{~mm}$; Hilgenberg, Malsfeld, Germany) and broken to a tip diameter matching to the antenna/maxillary palp stump. The dye-filled glass micropipette was put on the antenna/maxillary palp stump for about 4-6 hours in a moist chamber at $4{ }^{\circ} \mathrm{C}$. For the backfills of the whole mouthparts, the maxillary and labial palps were cut and the antennae were protected from unintentional dyefilling by covering them with dental wax (S-U-wax wire,
$2.0 \mathrm{~mm}$, hard). A crystal of biotin-conjugated dextran (3000 MW; Molecular Probes, Invitrogen) was placed onto the prepared mouthparts, covered with a drop of distilled water, and stored for about $4 \mathrm{~h}$ in a moist chamber at $4{ }^{\circ} \mathrm{C}$. Brains and ganglia were dissected, fixed, washed, and stained as described above. Neurobiotin was visualized with $\mathrm{Cy} 3$ conjugated streptavidin (Dianova, Hamburg, Germany) diluted $1 / 200$ in PBT (0.3 \% TrX). The staining solution contained in addition Alexa Fluor 488 -coupled phalloidin (1/200), DAPI $(1 / 20,000)$ and $2 \%$ NGS. The incubation time was $2-3$ days at $4{ }^{\circ} \mathrm{C}$. Biotincoupled dextran was visualized with Alexa Fluor 488coupled streptavidin (Molecular Probes, Invitrogen) diluted $1 / 200$ in PBT (0.3 \% TrX and $2 \%$ NGS) and applied together with synapsin $(1 / 300)$ for $2-3$ days at $4{ }^{\circ} \mathrm{C}$.

\section{In vivo dye injection into the antennal lobes}

Cold anesthetized animals with fluorescent labeled ALs (partial Orco-Gal4/UAS-DsRed) were mounted with their ventral side pointing upside down with dental wax on a microscope slide. The pronotum and the head capsule were opened using a piece of a razor blade held by a blade breaker, with two parallel longitudinal cuts along the compound eyes. The cuticle, fat tissue, and tracheae were removed. Afterwards, the head capsule and pronotum were covered with ringer solution [197]. A tungsten needle was sharpened in $2 \mathrm{M} \mathrm{KOH}$ with 5-8 volts as described in [198], followed by coating with Texas Red conjugated dextran (3000 MW; Molecular Probes, Invitrogen) dissolved in NGS and air-dried. The injection of dye into the DsRed-labeled AL was performed manually under a fluorescence stereomicroscope (SteREO Lumar.V12, Carl Zeiss MicroImaging, Jena, Germany) by careful perforation. The treated animals were kept in a moist chamber for about $1 \mathrm{~h}$ at room temperature to let the dye diffuse. Afterwards, the brains were dissected, fixed, washed, and pre-incubated with NGS as described previously and afterwards incubated with Alexa Fluor 488-coupled phalloidin $(1 / 200)$, DAPI $(1 / 20,000)$, and $2 \%$ NGS for 2 days at $4{ }^{\circ} \mathrm{C}$. Subsequently, the brains were washed, dehydrated, cleared, and mounted in Permount as described above.

\section{Microscopic image acquisition, processing, and analysis}

The fluorescent-labeled microscopic samples were scanned with CLSM (TCS SP5, Leica Microsystems) at $1024 \times 1024$ or $2048 \times 2048$ pixel resolution, a scanning speed between 100 and $200 \mathrm{~Hz}$, a pinhole of size 1 airy, a line average of $2-4$, and a step size between 0.5 and $2.5 \mu \mathrm{m}$. Confocal images and image stacks were analyzed with the Amira 5.3.3 graphics software (FEI, Hillsboro, OR, USA). The final image processing and figure arrangements were processed using Corel Draw X3 (Corel, 
Ottawa, Ontario, Canada), Adobe Photoshop CS3 (Adobe Systems, San Jose, CA, USA), or Inkscape [199].

The number of CSNs per sensillum was determined based on high-resolution CLSM stacks taken from antennae of the EF1-B-DsRed line after antibody enhancement of the DsRed reporter signal in combination with Orco antibody staining. To determine the number of CSNs and Orco-immunoreactive OSNs, we traced the stained dendrites of the CSNs to their associated soma of several sensilla and calculated their average number (Additional file 3: Figure S3i; Fig. 2h).

AL glomeruli were separately labeled in the AMIRA Segmentation Editor and 3D reconstructed [102, 200] based on CLSM stacks of brains labeled with synapsin and TKRP antibodies of five male and five female A7 beetles (one AL from a random hemisphere for each brain). To optimize data quality, the CLSM stacks were previously deconvoluted in AMIRA using the blind method with initial estimation set to input data, with a border width of 10,10 , and 10 , and an iteration of ten cycles.

KCs were identified based on their position, size, and density in DAPI stainings [103]. The total volumes of the whole CAs (13 CAs of seven A7 males), as well as the volumes of three randomly assigned clusters of $20 \mathrm{KCs}$ per $\mathrm{CA}$, were measured using $3 \mathrm{D}$ reconstruction. For the segmentation and reconstruction details, we refer to [201, 202]. Briefly, different layers of a structure were labeled in the Segmentation Editor and wrapped. Volumes of reconstructed structures were taken from Material Statistics. Based on the ratios between whole CA volume and the volumes of the three clusters of $20 \mathrm{KCs}$, the total number of $\mathrm{KCs}$ per CA was interpolated. In addition, we counted the KCs by an independent method using MorphoGraphX (www.MorphoGraphX.org). The CLSM stacks were processed with the arithmetic tool of AMIRA to mask the CAs and consequently to remove the remaining materials. The resulting stacks were converted to TIFF files with FIJI [203] by preserving the image properties. These files were analyzed with the "Local Maxima" tool of MorphoGraphX [111] with the following parameter X-/Y-/Z-radius $=1 \mu \mathrm{m}$, Start Label -1, and Min Color 1. Because of the inhomogeneous intensity distribution within some CLSM stacks, only nine CAs from five specimens were analyzed automatically.

Statistical analysis included determination of mean values, standard deviations, and independent/unpaired two-tailed Student's $t$-tests, which were performed in Excel XP (Microsoft, Redmond, WA, USA). Bar charts were created in Excel XP and imported and revised in Corel Draw.

\section{RNA isolation and sequencing}

Total RNA of about 1000 antennae, 150 mouthparts (a piece of the head capsule anterior of the antennae), 600 legs, 50 heads (without antennae but including mouthparts), and 20 remaining bodies of sex-separated adults was isolated using the ZR Tissue and Insect RNA Micro Prep Kit (Zymo Research, Irvine, CA, USA), following the manufacturer's protocol. The library preparations for RNA-Seq were performed using the TruSeq RNA Sample Preparation Kit (Illumina, San Diego, CA, USA) and CDNA libraries were amplified and sequenced using the cBot and HiSeq2000 from Illumina (paired end; $2 \times$ $100 \mathrm{bp}$ ). Biological triplicates of female and male antennae were sequenced. For the other tissues, one male and one female sample were used and one additional mouthpart sample was obtained from unsexed beetles. The number of biological replicates was chosen based on previous publications using similar approaches [128, 170, 171]. Each biological replicate came from independently prepared and processed tissue. No technical replicates were performed. For details, see Dippel et al. [89].

\section{Re-annotation of olfactory genes}

For manual inspection the obtained reads were mapped against the T. castaneum 4.0 genome using BLAT [204] and a genome browser was set up (http://bioinf.unigreifswald.de/tcas/). In a genome independent approach, a de novo assembly was built with Trinity (release 2013_08_14) [205] as described in [89]. The previously published OR [115], GR [81], IR [114], and SNMP [116, 117] sequences were used for further analysis. To identify the potential ODEs, the official (OGS3) [81-83], the preliminary AU2 and AU3, and the NCBI [206, 207] gene sets were used and a protein functional analysis was conducted using InterProScan [208]. All genes belonging to a protein family containing known ODEs in other insect species were collected (namely, ALDH, ALOX, CES, EH, GST, and CYP [4]). The redundant genes were removed and the sequences were reviewed. The identified GSTs and CYPs were collated to already published sequences $[148,150]$ and the names were adapted. For all other candidates, a genome-based name was built reflecting the protein family and the chromosomal localization (e.g., CES2D is the fourth CES on the second chromosome). The genes supposed to be involved in olfactory transduction of $D$. melanogaster were taken from [46], the corresponding sequences were downloaded from the Flybase [209], and the T. castaneum orthologs were identified by pBLAST embedded in the genome browser (http://bioinf.uni-greifswald.de/tcas/).

The revision of the olfactory genes was performed in an iterative process based on sequence comparison with the de novo assembly and the RNA-seq based gene annotations (AU3), a conserved domain search [210], and manual inspection of the aligned reads in the genome browser. For discrepancies, the gene models were manually curated. Finally, the chromosomal localization of the 
olfactory genes was determined by pBLAST against the genome assembly Tcas4.0. The ODE candidates were searched for signal peptides using the SignalP4.1 server [211]. The sequences and read numbers are summarized in Additional file 8: Table S1. The complete dataset including all relevant parameters has been deposited in the National Center for Biotechnology Information (NCBI) database repository 'Gene Expression Omnibus' (GEO accession number: GSE63162).

\section{Tribolium castaneum expression profiling}

The olfactory genes were identified in the AU3 gene set by pBLAST and the corresponding gene models were replaced with the re-annotated candidate sequences. The resulting enhanced AU3 gene set was used to map the RNAseq data with Bowtie2 [212] using the very sensitive presetting. The mapped reads were counted with Samtools [213] and normalized as RPKM values. The RPKMs were visualized (matrix2png interface, version 1.2.1; [214]) and the figures were arranged in Inkscape [199]. One male antennae sample was excluded from the subsequent analysis due to massive differences with the other five antennae samples in the principal component as well as cluster analysis. Because no significant differences for the genes of interest were observed between male and female reads, the sequenced tissues were considered as biological replicates. Statistical analysis was performed in $\mathrm{R}$ [215] with the DESeq package (version 1.12.0) [216] from Bioconductor [217]. $p$ values were calculated with a negative binomial test using raw read counts and adjusted for multiple testing with the Benjamini-Hochberg method. All tissues were compared to body as reference. Significant differentially expressed genes (false discovery rate $<0.05$ ) are marked with asterisks in the heat maps. However, since only two body samples served as controls, all conclusions from this should be treated as preliminary. Genes with an $R P K M \geq 0.5$ were considered as specifically expressed in the tissue. The tissue comparison was visualized as Venn diagrams (e.g., Additional file 9: Figure S5a) (http://bioinformatics.psb.ugent.be/webtools/Venn/). For details, see Dippel et al. [89].

\section{Phylogenetic analysis and interspecies comparison}

We compared the $T$. castaneum IR, GR, and OR sequences independent from each other on a protein level with data from D. melanogaster [46, 209] and An. gambiae $[128,218]$. The sequences were aligned using MAFFT (v7.040b [219]) (-genafpair -maxiterate 1000 -bl 62 -op 1.53 -ep 0.123) and the phylogeny was calculated using RAxML (version 7.8.6 [220]), with the LG substitution model and GAMMA correction. The robustness of the tree topology was evaluated by 100 rapid bootstrap replications. The relative expression levels were calculated as $\log _{2}$-fold changes of antenna/body and palp (mouthpart)/ body as described in [89]. The D. melanogaster data set was downloaded from the EMBL gene expression atlas [221], originally published in [170], and the An. gambiae data were obtained from [128]. The phylogenetic tree was visualized by iTOL [222] and descriptions were added using Inkscape [199].

\section{Additional files}

Additional file 1: Figure S1. Antibody staining against DsRed and Orco of the EF1-B-DsRed line. a Maximum projection of a confocal image stack of a halved antenna of the EFT-B-DsRed line, with an antibody staining against DsRed and Orco and in addition DAPI. Showing Orco

immunoreactivity in the last three segments and particularly in the SBas. The DsRed reporter line labels in addition the scolopidia cells of Johnston's organ $(J O)$ in the pedicellus $(P)$. S, scapus. b Optical section of a mechano- and chemosensillum trichoideum (mSTri and CSTri) labeled with an Orco antibody (green) shows immunoreactivity only within the sensillum cavity of the cSTri. Autofluorescence of the cuticle at $560 \mathrm{~nm}$ is in blue. $\mathbf{c}-\mathbf{c}^{\prime \prime}$ Single optical section of a sensilla basiconica (SBas) in the EFT-B-DsRed (magenta, $\mathbf{b}^{\prime}$ ) line labeled with an Orco antibody (green, $\mathbf{b}^{\prime \prime}$ reveals signals of both channels particularly within the cavity and at the base of the sensillum. Both channels also show autofluorescence of the cuticle. $\mathbf{d}$-d" Optical section of two sensilla coeloconica (SCoe) in the EF1-B-DsRed (magenta, $\mathbf{d}^{\prime}$ ) line labeled with an Orco antibody (green, d") reveals no specific immunoreactivity within the sensilla cavities. Both channels also show autofluorescence of the cuticle. (TIF $8703 \mathrm{~kb}$ )

Additional file 2: Figure S2. Orco localization in antennae and palp. a Fluorescent in situ hybridization against Orco in the club segments in a maximum projection of a halved antenna. $\mathbf{b}, \mathbf{c}$ Specificity of the Orco antibody. IHC against Orco in antennae of $\mathbf{b}$ a San Bernadino beetle after Orco ${ }^{\mathrm{RNAi}}$ treatment (light cuticle, inset in the left lower corner) and $\mathbf{c}$ an untreated control included in the IHC (black strain identified by dark cuticle). The cross-reactive Orco antiserum results in no detectable staining in the antenna after Orco knockdown, whereas in the antenna of the untreated beetles, the odorant receptor neurons (ORNs) are clearly labeled by the antiserum (magenta). This indicates the specificity of the Orco antiserum against TcasOrco. Counterstaining with phalloidin (green) and DAPI (blue). The Orco antibody staining was labeled with a goat anti rabbit Cy3 secondary antibody. d-d" Immunohistochemical characterization of the Orco-Ga/4 line in the antenna and brain. Double immune-staining against Orco (magenta) and tGFP (green) in the antennae of the partial Orco-Gal4/UAS-tGFP line revealed that only half of the Orco-immunoreactive neurons expressing tGFP (arrow indicates colocalization and arrowhead as an example for no colocalization). e Immunohistochemical characterization of the Orco-Gal4 line in the brain Antibody staining against tGFP in the Orco-Gal4/UAS-tGFP line (orange) labels only half of the AL glomeruli represented as a 3D reconstruction (light green, based on a phalloidin staining). AL glomeruli not labeled are not shown. $\mathbf{f} \mid$ mmunohistochemical characterization of the partial Orco-Gal4 line in the palps. Double immuno-staining against Orco (green) and dsRed (magenta) in the palps of the Orco-Gal4/UAS-dsRed line reveals that all genetically labeled neurons are also Orco-immunoreactive. However, in contrast to the antennae, in which about half of the Orco-immunoreactive neurons are labeled in the partial Orco-Ga/4 line, only a few of the the Orcoimmunoreactive odorant receptor neurons express the reporter in the palps. The Cy2 (Orco) signal is quenched by dsRed. (TIF $6221 \mathrm{~kb}$ )

Additional file 3: Figure S3. Comparison of sensilla type numbers on the antenna of Tribolium castaneum and chemosensory neurons entering the sensilla types. a-e Number of different sensilla types on the 11th segment of the antenna: a sensilla campaniformis (Scam: 0 5.4; SD 1.8; of 5.4; SD 0.8), b spatulate bristles (SpaB: ô 10.2; SD 0.9; + 10.3; SD 0.9), c mechanoreceptive sensilla trichoideum (mSTri: 36.9; SD 3; 9 37.6; SD 4.3), d chemoreceptive sensilla trichoideum (cSTri: ô 86.3; SD 9.3; 9 87.1; SD 6.9), e sensilla coeloconica (SCoe: $\delta$ 6.8; SD 1.4; 우 7.6; SD 1.1). f Amount of sensilla basiconica on the club segments (11th: 24. SD 1.5: 0 25.5; SD 1.3; tenth: of 16.2; SD 1.5; ㅇ 16.4; SD 1.4; ninth: of 13.6; SD 0.9; 우 13.8; 
SD 1.1), regardless of the number of prongs. $\mathbf{g}$ Number of sensilla basiconica as in (f), but considering the prong number. $\mathbf{h}$ Number of different sensilla in the lateral corner of the tenth and ninth segments. $\mathbf{i}$ Number of chemosensory neurons (CSNs) entering the chemoreceptive sensilla: SBas 5.92 CSNs per prong ( $S D=1.2 ; n=73$ prongs of total 48 SBas), CSTri 1.07 CSNs (SD $=0.25 ; n=61)$, and SCoe 3.16 CSNs (SD = 1.10; $n=5)$. Error bars represent standard deviations; $n=$ number of antennae. (TIF $1147 \mathrm{~kb}$ )

Additional File 4: Movie S1. 3D reconstructions of the antennal nerve, antennal lobe, antennal mechanosensory and motor center, and lobus glomerulatus. Z-stack video of a phalloidin stained brainwith embedded 3DReconstruction of antennal lobe (dark blue), antennal nerve (light blue), antennal mechanosensory and motor center (turquoise), and the lobus glomerulatus (magenta). Later the neuropils are embedded in a voltex projection of the brain, also based on phalloidin staining (orange). (MP4 $11380 \mathrm{~kb}$ )

Additional file 5: Movie S2. Camera path through a confocal stack of the AL with backfills of the antenna and maxillary palp.Obtained from the same confocal stack as Figure 3A. Antennal backfill in green and maxillary palp in magenta. (MP4 $5186 \mathrm{~kb})$

Additional file 6: Figure S4. Ipsilateral antennal projection. Maximum intensity projection of a brain labeled with an antibody against synapsin (green) and a neurotracer resulting from an antennal backfill (magenta). The antennal backfill labels exclusively structures in the ipsilateral hemisphere, mainly the AL via the antennal nerve $(*)$, and a tract (arrowhead) descending to the gnathal ganglion The inset depicts a projection of only a few optical sections showing fibers interconnecting the $\mathrm{AL}$ and the protocerebrum with some arborizations in the accessory medulla of the optical lobe (arrow) suggesting an integration of circadian information. (TFF $3816 \mathrm{~kb}$ )

Additional File 7: Movie S3. Voltex projection of the gnathal ganglion and part of the brain of the Orco-Gal4/UAS-DsRed line. The video was obtained from the same confocal stack as Figure 3F. It shows two paired input tracts from the maxillary and labial palps, that converge in GOC and ascend to the LGs, as well as the partially labeled ALs. (MP4 $16701 \mathrm{~kb}$ )

Additional file 8: Table S1. Summary of the RNAseg data. In column (A) the gene name of the GRs according to [81], the ORs from [115], the SNMPs modified after $[116,117]$, the $T$. castaneum orthologous of $D$. melanogaster genes named after candidates obtained from [46], the GSTs named after [148], the CYPs named after [150] and the remaining ODE candidates de novo named according to their chromosomal localization (Additional file 15: Figure S11). Column B shows the sequences of the ORF based on published annotations or existing gene models, but modified if necessary. Confirmed gene models are highlighted in grey, modified ones are highlighted in yellow, only partially covered but expressed ones are highlighted in orange, genes with low and scattered coverage are highlighted in red, and not highlighted sequences were not manually checked. Columns C-I show the average RPKM values of antennae, mouthparts (piece of the head capsule anterior of the antennae), legs, head (without antennae but including mouthparts), and remaining body of sex-separated adult animals. Columns J-Q show the results of the statistica analysis conducted in R [215] with the DESeq package (version 1.12.0) [216] (from Bioconductor [217], based on five antenna samples and two replicates for the other adult tissues in comparison to body. Columns P-AB show the individual RPKM values of the biological replicates. (XLSX $677 \mathrm{~kb}$ )

Additional file 9: Figure S5. $I R$ gene tissue expression and chromosomal localization of IR and SNMP genes. a Venn diagram showing the number of IRs expressed (RPKM $\geq 0.5$ ) in the different body parts: antennae, legs, mouthparts (as a piece of the head capsule anterio of the antennae), heads (the whole head capsule including mouthparts but excluding the antennae), and bodies (excluding head and legs). $\mathbf{b}$ Based on Georgia GA-2 strain genome assembly 3 [81], only chromosomal linkage groups containing an IR or SNMP are depicted. Gene clusters are indicated by a number referring to the chromosome and a letter conveys the relative position on the chromosome. The number of genes within this cluster is indicated in square brackets. (PDF $66 \mathrm{~kb}$ )

Additional file 10: Figure S6. Phylogenetic tree of IRs. Outer rings represent the expression in body, mouthparts ( $T$. castaneum: palps, mandible, labrum, and labium; D. melanogaster: palp and proboscis; An. gambiae: maxillary palp) and antenna as a percentage compared to the highest expressed gene according to the scale in the left upper corner. Note that the methods used to obtain the different expression data (RNAseq and microarray) are not directly comparable. This figure can, thus, only give an impression of the tissue-specific abundance of the transcripts. The scale bars within the trees represent one amino acid substitution per site. Antennal IRs are highlighted in yellow. (PDF 625 kb)

Additional file 11: Figure S7. $G R$ gene tissue expression and their chromosomal localization. a Venn diagram showing the number of GRs expressed (RPKM $\geq 0.5$ ) in the different body parts: antennae, legs, mouthparts (as a piece of the head capsule anterior of the antennae), heads (the whole head capsule including mouthparts but excluding the antennae), and bodies (excluding head and legs). b Based on Georgia GA-2 strain genome assembly 3.0 [81], only chromosomal linkage groups containing an IR or SNMP are depicted. Gene clusters are indicated by a number referring to the chromosome and a letter conveys the relative position on the chromosome. The number of genes within this cluster is indicated in the square brackets. (PDF $176 \mathrm{~kb}$ )

Additional file 12: Figure S8. Phylogenetic mid-point rooted tree of the GRs based on protein sequences. Outer rings represent the expression in body, mouthparts (T. castaneum: palps, mandible, labrum, and labium; $D$. melanogaster: palp and proboscis; An. gambiae: maxillary palp) and antenna as a percentage compared to the highest expressed gene according to the scale in the left upper corner. Note that the methods used to obtain the different expression data (RNAseq and microarray) are not directly comparable. This figure can, thus, only give an impression of the tissue-specific abundance of the transcripts. The scale bars within the trees represent 1 amino acid substitution per site. Potential sugar and fructose receptors are labeled and highlighted in yellow and in grey, and $\mathrm{CO}_{2}$ receptors are highlighted in orange. (PDF $1733 \mathrm{~kb}$ )

Additional file 13: Figure S9. $O R$ gene tissue expression and their chromosomal localization. a Venn diagram showing the number of ORs expressed (RPKM $\geq 0.5$ ) in the different body parts: antennae, legs, mouthparts (as piece of the head capsule anterior of the antennae), heads (the whole head capsule including mouthparts but excluding the antennae), and bodies (excluding head and legs). b Venn diagram comparing our results (yellow, green) with data from Engsontia et al. [115] (blue, red). Number of expressed ORs, defined by RPKM $\geq 0.5$ (yellow), by RT-PCR (blue), not expressed RPKM $<0.5$ (green), or with no RT-PCR amplicon (red). ORs of the brown group were not previously tested by Engsontia et al. c Chromosomal localization of T. castaneum ORs. Based on the Georgia GA-2 strain genome assembly 3.0 [81], only chromosomal linkage groups containing an IR or SNMP are depicted. Gene clusters are indicated by a number referring to the chromosome and a letter conveys the relative position on the chromosome. The number of genes within this cluster is indicated in the square brackets. (PDF $277 \mathrm{~kb}$ )

Additional file 14: Figure S10. Phylogenetic tree of the ORs based on protein sequences. Outer rings represent the expression in body, mouthparts (T. castaneum: palps, mandible, labrum, and labium; D. melanogaster: palp and proboscis; An. gambiae: maxillary palp) and antenna as a percentage compared to the highest expressed gene according to the scale in the left upper corner. Note that the methods used to obtain the different expression data (RNAseq and microarray) are not directly comparable. This figure can, thus, only give an impression of the tissue-specific abundance of the transcripts. The scale bars within the trees represent one amino acid substitution per site. (PDF $2302 \mathrm{~kb}$ )

Additional file 15: Figure S11. Chromosomal localization of potential T. castaneum ODE genes. Based on Georgia GA-2 strain genome assembly 3.0 [81], aldehyde dehydrogenase (ALDH, in grey), aldehyde oxidase (ALOX, in orange), carboxylesterase (CES, in blue), epoxide hydrolase (EH, in green), glutathione S-transferase (GST, in purple), and cytochrome P450 (CYP, in magenta). Gene clusters are indicated by a number referring to the chromosome and a letter conveys the relative position on the chromosome. The number of genes within this cluster is indicated in the square brackets. (PDF $257 \mathrm{~kb}$ )

Additional file 16: Sequences of primers and template plasmid used to generate pSLfa1180[2.5kbOrcoUp_GAL4delta]. (PDF $111 \mathrm{~kb}$ )

Additional file 17: Figure S12. UAS responder lines in the absence of Gal4 driver. In the four rows, the maximum projections of head capsules from different transgenic strains (Orco-Gal4/UAS-tGFP, UAS-tGFP, Orco-Gal4/ UAS-dsRed, and UAS-dsRed) are depicted. The upper row represents the overlay of both channels (GFP/YFP in green and dsRed in red). For UAS 
responders without the Gal4 driver, high-resolution images of the palps and the antennal club are provided. In the second and third rows, the separated channels are given as greyscale images. The UAS-tGFP and UAS-dsRed lines do not show leaky reporter expression in the absence of a Gal4 driver in the antennae and palps. The presence of the genetic constructs is indicated by the eye markers: pBac[3XP3-dsRed_UAS-Tchsp68bP-tGFP-SV40] and pBac[3XP3-eYFP_UAS-Tchsp68bP-DsRedex-SV40]. The marker signal is quenched in the crossed lines by the vermillion rescue marker of the Orco-Gal4 construct pBac[3XP3-gVerm_2.5kbOrcoUp_GAL4delta]. (TIF 6507 kb)

Additional file 18: Table S2. Primary and secondary antibodies and dyes used with additional information such as source and specificity. $n / a$ not available. (PDF $118 \mathrm{~kb}$ )

\section{Abbreviations}

AL: Antennal lobe; ALDH: Aldehyde dehydrogenase; ALOX: Aldehyde oxidase; ALT: Antennal lobe tract; AMMC: antennal mechanosensory and motor center; AN: antennal nerve; CA: Calyx of the MB; CES: Carboxylesterase; CLSM: Confocal laser-scanning microscopy; CSN: Chemosensory neuron; CSP: Chemosensory protein; CSTri: Chemosensilla trichoidea;

CYP: Cytochrome P450; EH: Epoxide hydrolase; GABA: Gamma-aminobutyric acid; GL: Antennal lobe glomeruli; GNG: Gnathal ganglia; GOC: Gnathal olfactory center; GR: Gustatory receptor; GSN: Gustatory sensory neuron; GST: Glutathione S-transferase; IHC: Immunohistochemistry; IR: Ionotropic glutamate-like receptor; KC: Kenyon cells; IALT: Lateral antennal lobe tract; LG: Lobus glomerulatus; LH: Lateral horn (lateral protocerebrum); LN: Local interneuron; mALT: Mediolateral lobe tract; MB: Mushroom body; mIALT: Mediolateral antennal lobe tract; mSTri: Mechanosensilla trichoidea; NA: Neuro-axis; NGS: normal goat serum; OBP: Odorant binding protein; ODE: Odorant degrading enzyme; OR: Odorant receptor; Orco: Odorant receptor co-receptor; ORF: Open reading frame; OSN: Olfactory sensory neuron; PBS: Phosphate buffered saline; PGC: Primary gustatory center; PN: Projection neuron; RPKM: Reads per kilobase per million; SBas: Sensilla basiconica; SCam: Sensilla campaniformes; SCha: Sensilla chaetica; SCoe: Sensilla coeloconica; SD: standard deviation; SEM: Scanning electron microscopy; SNMP: Sensory neuron membrane protein; SpaB: Spaculate bristle; TKRP: Tachykinin-related peptide

\section{Acknowledgements}

We thank the transcriptome analysis laboratory of the University Medical Center Göttingen, Germany, especially Gabriela Salinas-Riester for sequencing and technica support; Jürgen Krieger for providing a cross-reactive previously unpublished Orco antibody; Gregor Bucher and Michalis Averof for sharing transgenic beetle lines and plasmids; Hugh M. Robertson and Kimberly K O. Walden for providing unpublished sequence information; Mario Stanke und Lizzy Gerischer for the iBeetle Genome Browser; Richard Smith for support on MorphoGraphix; Martina Kern, Marlene Binzer, and Peter Christ for technical assistance; Montserrat Torres Oliva and Jan Kropf for technical advice; Kei Ito for comments on the manuscript; as well as Uwe Homberg, and the members of the Deutsche Forschungsgemeinschaft Priority Program SPP 1392 "Integrative Analysis of Olfaction" for a fruitful discussion.

\section{Funding}

Deutsche Forschungsgemeinschaft SPP 1392: SCHA 678/13-1 (JS) and W 1797/4-1 (EAW). The funders had no role in the study design, data collection, interpretation, or the decision to submit the work for publication.

\section{Availability of data and materials}

Previously Published Dataset: Tissue-specific transcriptomics, chromosomal localization, and phylogeny of chemosensory and odorant binding proteins from the red flour beetle Tribolium castaneum reveal subgroup specificities for olfaction or more general functions: Wimmer EA, Dippel S, Oberhofer G, 2015, http:/www.ncbi.nlm.nih.gov/geo/query/acc.cgi?acc=GSE63162, GSE63162.

\section{Authors' contributions}

SD and MK conceived and designed the study; acquired, analyzed, and interpreted the data; and drafted and revised the article. GO and RK analyzed, and interpreted the data. AM acquired, analyzed, and interpreted the data; and revised the article. CK and MK acquired the data and revised the article. KR acquired the data. SF acquired, analyzed, and interpreted the data. JS and EAW conceived and designed the study; analyzed and interpreted the data; and drafted and revised the article. All authors read and approved the final manuscript.

\section{Competing interests}

The authors declare that they have no competing interests.

\section{Author details}

${ }^{1}$ Department of Developmental Biology, Göttingen Center for Molecular Biosciences (GZMB), Georg-August-University Goettingen, Johann-Friedrich-Blumenbach-Institute of Zoology and Anthropology, Ernst-Caspari-Haus, Justus-von-Liebig-Weg 11, 37077 Göttingen, Germany. ${ }^{2}$ Department of Biology - Neurobiology/Ethology, Philipps-University Marburg, Karl-von-Frisch-Str. 8, 35032 Marburg, Germany. ${ }^{3}$ Department of Evolutionary Developmental Genetics, GZMB, Ernst-Caspari-Haus, Georg-August-University Goettingen, Johann-Friedrich-Blumenbach-Institute of Zoology and Anthropology, Justus-von-Liebig-Weg 11, 37077 Göttingen, Germany. ${ }^{4}$ Department of Biology - Mycology, Philipps-University Marburg, Karl-von-Frisch-Str. 8, 35032 Marburg, Germany. ${ }^{5}$ Department of Plant Systems Biology, Flanders Institute for Biotechnology, Technologiepark 927, 9052 Gent, Belgium.

Received: 30 August 2016 Accepted: 5 September 2016

Published online: 17 October 2016

\section{References}

1. de Bruyne M, Smart R, Zammit E, Warr CG. Functional and molecular evolution of olfactory neurons and receptors for aliphatic esters across the Drosophila genus. J Comp Physiol A Neuroethol Sens Neural Behav Physiol. 2010;196:97-109.

2. Dahanukar A, Hallem EA, Carlson JR. Insect chemoreception. Curr Opin Neurobiol. 2005;15:423-30.

3. Tegoni M, Campanacci V, Cambillau C. Structural aspects of sexual attraction and chemical communication in insects. Trends Biochem Sci. 2004;29:257-64.

4. Leal WS. Odorant reception in insects: roles of receptors, binding proteins, and degrading enzymes. Annu Rev Entomol. 2013;58:373-91.

5. Visser JH. Host odor perception in phytophagous insects. Annu Rev Entomol. 1986;31:121-44

6. Whiteman NK, Pierce NE. Delicious poison: genetics of Drosophila host plant preference. Trends Ecol Evol. 2008:23:473-8.

7. Liu M, Yu H, Li G. Oviposition deterrents from eggs of the cotton bollworm, Helicoverpa armigera (Lepidoptera: Noctuidae): chemical identification and analysis by electroantennogram. J Insect Physiol. 2008:54:656-62

8. Sun Y-L, Huang L-Q, Pelosi P, Wang C-Z. Expression in antennae and reproductive organs suggests a dual role of an odorant-binding protein in two sibling Helicoverpa species. PLoS One. 2012;7, e30040.

9. Dicke M. Behavioural and community ecology of plants that cry for help. Plant Cell Environ. 2009:32:654-65.

10. Stensmyr MC, Dweck HKM, Farhan A, Ibba I, Strutz A, Mukunda L, et al. A conserved dedicated olfactory circuit for detecting harmful microbes in Drosophila. Cell. 2012:151:1345-57.

11. Laska M, Galizia CG, Giurfa M, Menzel R. Olfactory discrimination ability and odor structure-activity relationships in honeybees. Chem Senses. 1999;24:429-38.

12. Yang $\mathrm{C}-\mathrm{H}$, Belawat $\mathrm{P}$, Hafen $\mathrm{E}$, Jan $\mathrm{LY}$, Jan $\mathrm{Y}-\mathrm{N}$. Drosophila egg-laying site selection as a system to study simple decision-making processes. Science. 2008:319:1679-83.

13. Weiss LA, Dahanukar A, Kwon JY, Banerjee D, Carlson JR. The molecular and cellular basis of bitter taste in Drosophila. Neuron. 2011:69:258-72.

14. Linz J, Baschwitz A, Strutz A, Dweck HKM, Sachse S, Hansson BS, et al. Host plant-driven sensory specialization in Drosophila erecta. Proc R Soc Lond B Biol Sci. 2013:280:20130626

15. Paczkowski S, Paczkowska M, Dippel S, Flematti G, Schütz S. Volatile combustion products of wood attract Acanthocnemus nigricans (Coleoptera: Acanthocnemidae). J Insect Behav. 2014;27:228-38.

16. Steinbrecht RA. Structure and function of insect olfactory sensilla. Ciba Found Symp. 1996:200:158-77.

17. de Bruyne M, Warr CG. Molecular and cellular organization of insect chemosensory neurons. Bioessays. 2006;28:23-34.

18. Pelosi P, lovinella I, Felicioli A, Dani FR. Soluble proteins of chemical communication: an overview across arthropods. Front Physiol. 2014;5:320.

19. Benton R, Vannice KS, Gomez-Diaz C, Vosshall LB. Variant ionotropic glutamate receptors as chemosensory receptors in Drosophila. Cell. 2009;136:149-62.

20. Rytz R, Croset V, Benton R. lonotropic receptors (IRs): chemosensory ionotropic glutamate receptors in Drosophila and beyond. Insect Biochem Mol Biol. 2013;43:888-97. 
21. Montell C. A taste of the Drosophila gustatory receptors. Curr Opin Neurobiol. 2009;19:345-53.

22. Sato K, Pellegrino M, Nakagawa T, Nakagawa T, Vosshall LB, Touhara K. Insect olfactory receptors are heteromeric ligand-gated ion channels. Nature. 2008;452:1002-6.

23. Wicher D, Schäfer R, Bauernfeind R, Stensmyr MC, Heller R, Heinemann SH, et al. Drosophila odorant receptors are both ligand-gated and cyclicnucleotide-activated cation channels. Nature. 2008;452:1007-11.

24. Missbach C, Dweck HK, Vogel H, Vilcinskas A, Stensmyr MC, Hansson BS, et al. Evolution of insect olfactory receptors. eLife. 2014;3, e02115.

25. Sánchez-Gracia A, Vieira FG, Almeida FC, Rozas J. Comparative Genomics of the Major Chemosensory Gene Families in Arthropods. eLS. 2011. doi:10. 1002/9780470015902.a0022848.

26. Cao TNP. Genome annotation and evolution of chemosensory receptors in spider mites. Dissertation. Ghent University; 2014.

27. Chipman AD, Ferrier DEK, Brena C, Qu J, Hughes DST, Schröder R, et al. The first myriapod genome sequence reveals conservative arthropod gene content and genome organisation in the centipede Strigamia maritima. PLoS Biol. 2014;12, e1002005.

28. Chyb S. Drosophila gustatory receptors: from gene identification to functional expression. J Insect Physiol. 2004;50:469-77.

29. Freeman EG, Wisotsky Z, Dahanukar A. Detection of sweet tastants by a conserved group of insect gustatory receptors. Proc Natl Acad Sci. 2014;111:1598-603.

30. Lee $Y$, Moon SJ, Montell C. Multiple gustatory receptors required for the caffeine response in Drosophila. Proc Natl Acad Sci. 2009;106:4495-500.

31. Jiao Y, Moon SJ, Wang X, Ren Q, Montell C. Gr64f is required in combination with other gustatory receptors for sugar detection in Drosophila. Curr Biol. 2008;18:1797-801.

32. Ishimoto $H$, Takahashi $K$, Ueda R, Tanimura T. G-protein gamma subunit 1 is required for sugar reception in Drosophila. EMBO J. 2005;24:3259-65.

33. Sato $K$, Tanaka $K$, Touhara K. Sugar-regulated cation channel formed by an insect gustatory receptor. Proc Natl Acad Sci. 2011;108:11680-5.

34. Smart R, Kiely A, Beale M, Vargas E, Carraher C, Kralicek AV, et al. Drosophila odorant receptors are novel seven transmembrane domain proteins that can signal independently of heterotrimeric $\mathrm{G}$ proteins. Insect Biochem Mol Biol. 2008;38:770-80.

35. Mukunda L, Lavista-Llanos S, Hansson BS, Wicher D. Dimerisation of the Drosophila odorant coreceptor Orco. Front Cell Neurosci. 2014;8:261.

36. German PF, van der Poel S, Carraher C, Kralicek AV, Newcomb RD. Insights into subunit interactions within the insect olfactory receptor complex using FRET. Insect Biochem Mol Biol. 2013:43:138-45.

37. Getahun MN, Olsson SB, Lavista-Llanos S, Hansson BS, Wicher D. Insect odorant response sensitivity is tuned by metabotropically autoregulated olfactory receptors. PLoS One. 2013;8, e58889.

38. Stengl M, Funk NW. The role of the coreceptor Orco in insect olfactory transduction. J Comp Physiol A. 2013;199:897-909.

39. Nolte A, Funk NW, Mukunda L, Gawalek P, Werckenthin A, Hansson BS, et al. In situ tip-recordings found no evidence for an Orco-based ionotropic mechanism of pheromone-transduction in Manduca sexta. PLoS One. 2013; 8, e62648.

40. Martin F, Alcorta E. Regulation of olfactory transduction in the Orco channel. Front Cell Neurosci. 2011;5:21.

41. Riesgo-Escovar J, Raha D, Carlson JR. Requirement for a phospholipase C in odor response: overlap between olfaction and vision in Drosophila. Proc Natl Acad Sci. 1995;92:2864-8.

42. Miura N, Atsumi S, Tabunoki H, Sato R. Expression and localization of three $\mathrm{G}$ protein a subunits, Go, Gq, and Gs, in adult antennae of the silkmoth (Bombyx mori). J Comp Neurol. 2005;485:143-52.

43. Chatterjee A, Roman G, Hardin PE. Go contributes to olfactory reception in Drosophila melanogaster. BMC Physiol. 2009;9:22.

44. Deng Y, Zhang W. Farhat K, Oberland S, Gisselmann G, Neuhaus EM. The stimulatory gas protein is involved in olfactory signal transduction in Drosophila. PLoS One. 2011;6, e18605.

45. Sargsyan V, Getahun MN, Llanos SL, Olsson SB, Hansson BS, Wicher D. Phosphorylation via PKC regulates the function of the Drosophila odorant co-receptor. Front Cell Neurosci. 2011;5:5.

46. Martin F, Boto T, Gomez-Diaz C, Alcorta E. Elements of olfactory reception in adult Drosophila melanogaster. Anat Rec. 2013:296:1477-88.

47. Ignatious Raja JS, Katanayeva N, Katanaev VL, Galizia CG. Role of Go/i subgroup of $\mathrm{G}$ proteins in olfactory signaling of Drosophila melanogaster. Eur J Neurosci. 2014:39:1245-55.
48. Jin X, Ha TS, Smith DP. SNMP is a signaling component required for pheromone sensitivity in Drosophila. Proc Natl Acad Sci. 2008;105:109961001.

49. Benton $R$, Vannice KS, Vosshall LB. An essential role for a CD36-related receptor in pheromone detection in Drosophila. Nature. 2007;450:289-93.

50. Li Z, Ni JD, Huang J, Montell C. Requirement for Drosophila SNMP1 for rapid activation and termination of pheromone-induced activity. PLoS Genet. 2014; 10, e1004600.

51. Maïbèche-Coisne M, Nikonov AA, Ishida Y, Jacquin-Joly E, Leal WS Pheromone anosmia in a scarab beetle induced by in vivo inhibition of a pheromone-degrading enzyme. Proc Natl Acad Sci. 2004;101:11459-64.

52. Ishida Y, Leal WS. Rapid inactivation of a moth pheromone. Proc Natl Acad Sci. 2005;102:14075-9.

53. Durand N, Carot-Sans G, Bozzolan F, Rosell G, Siaussat D, Debernard S, et al. Degradation of pheromone and plant volatile components by a same odorant-degrading enzyme in the cotton leafworm, Spodoptera littoralis. PLOS ONE. 2011;6, e29147.

54. Chertemps T, François A, Durand N, Rosell G, Dekker T, Lucas P, et al. A carboxylesterase, Esterase-6, modulates sensory physiological and behavioral response dynamics to pheromone in Drosophila. BMC Biol. 2012;10:56.

55. Younus F, Chertemps T, Pearce SL, Pandey G, Bozzolan F, Coppin CW, et al, Identification of candidate odorant degrading gene/enzyme systems in the antennal transcriptome of Drosophila melanogaster. Insect Biochem Mol Biol. 2014:53:30-43.

56. Scott $K$, Brady Jr R, Cravchik A, Morozov P, Rzhetsky A, Zuker C, et al. A chemosensory gene family encoding candidate gustatory and olfactory receptors in Drosophila. Cell. 2001;104:661-73.

57. Schachtner J, Schmidt M, Homberg U. Organization and evolutionary trend of primary olfactory brain centers in Tetraconata (Crustacea + Hexapoda). Arthropod Struct Dev. 2005;34:257-99.

58. Vosshall LB. Olfaction in Drosophila. Curr Opin Neurobiol. 2000;10:498-503.

59. Stocker RF. Drosophila as a focus in olfactory research: mapping of olfactory sensilla by fine structure, odor specificity, odorant receptor expression, and central connectivity. Microsc Res Tech. 2001;55:284-96.

60. Keller A, Vosshall LB. Decoding olfaction in Drosophila. Curr Opin Neurobiol. 2003;13:103-10.

61. Jefferis GSXE. Insect olfaction: a map of smell in the brain. Curr Biol. 2005;15 R668-70.

62. Smith CUM. Olfaction. In: Biology of sensory systems. John Wiley \& Sons; 2009. doi: $10.1002 / 9780470694374 . c h 14$.

63. Couto A, Alenius M, Dickson BJ. Molecular, anatomical, and functional organization of the Drosophila olfactory system. Curr Biol. 2005;15:1535-47.

64. Ernst DKD, Boeckh J, Boeckh V. A neuroanatomical study on the organization of the central antennal pathways in insects. Cell Tissue Res. 1977:176:285-308.

65. Ignell $\mathrm{R}$, Anton S, Hansson BS. The maxillary palp sensory pathway of Orthoptera. Arthropod Struct Dev. 2000;29:295-305.

66. Hofer S, Dircksen H, Tollbäck P. Homberg U. Novel insect orcokinins: characterization and neuronal distribution in the brains of selected dicondylian insects. J Comp Neurol. 2005;490:57-71.

67. Christensen TA, Waldrop BR, Harrow ID, Hildebrand JG. Local interneurons and information processing in the olfactory glomeruli of the moth Manduca sexta. J Comp Physiol A Neuroethol Sens Neural Behav Physiol. 1993;173: 385-99.

68. Chou Y-H, Spletter ML, Yaksi E, Leong JCS, Wilson RI, Luo L. Diversity and wiring variability of olfactory local interneurons in the Drosophila antennal lobe. Nat Neurosci. 2010;13:439-49.

69. Seki Y, Rybak J, Wicher D, Sachse S, Hansson BS. Physiological and morphological characterization of local interneurons in the Drosophila antennal lobe. J Neurophysiol. 2010;104:1007-19.

70. Galizia CG, Rössler W. Parallel olfactory systems in insects: anatomy and function. Annu Rev Entomol. 2010;55:399-420

71. Wang Y, Chiang A-S, Xia S, Kitamoto T, Tully T, Zhong Y. Blockade of neurotransmission in Drosophila mushroom bodies impairs odor attraction but not repulsion. Curr Biol. 2003:13:1900-4.

72. Jefferis GSXE, Potter CJ, Chan AM, Marin EC, Rohlfing T, Maurer Jr CR, et al. Comprehensive maps of Drosophila higher olfactory centers: spatially segregated fruit and pheromone representation. Cell. 2007;128: 1187-203.

73. Yamagata N, Nishino H, Mizunami M. Neural pathways for the processing of alarm pheromone in the ant brain. J Comp Neurol. 2007;505:424-42. 
74. Strutz A, Soelter J, Baschwitz A, Farhan A, Grabe V, Rybak J, et al. Decoding odor quality and intensity in the Drosophila brain. eLife. 2014;3, e04147.

75. de Belle J, Heisenberg M. Associative odor learning in Drosophila abolished by chemical ablation of mushroom bodies. Science. 1994:263:692-5.

76. Connolly JB, Roberts IJ, Armstrong JD, Kaiser K, Forte M, Tully T, et al. Associative learning disrupted by impaired Gs signaling in Drosophila mushroom bodies. Science. 1996;274:2104-7.

77. Heimbeck G, Bugnon V, Gendre N, Keller A, Stocker RF. A central neura circuit for experience-independent olfactory and courtship behavior in Drosophila melanogaster. Proc Natl Acad Sci. 2001;98:15336-41.

78. Morris SC, Grimaldi D, Engel MS. Evolution of the Insects. Cambridge, New York, Melbourne: Cambridge University Press; 2005. http://www.cambridge. org/catalogue/catalogue.asp?isbn=9780521821490\&ss=fro

79. Hunt T, Bergsten J, Levkanicova Z, Papadopoulou A, John OS, Wild R, et al. A comprehensive phylogeny of beetles reveals the evolutionary origins of a superradiation. Science. 2007;318:1913-6.

80. Brown SJ, Shippy TD, Miller S, Bolognesi R, Beeman RW, Lorenzen MD, et al. The red flour beetle, Tribolium castaneum (Coleoptera): a model for studies of development and pest biology. Cold Spring Harb Protoc. 2009;2009:pdb.emo126.

81. Richards S, Gibbs RA, Weinstock GM, Brown SJ, Denell R, Beeman RW, et al. The genome of the model beetle and pest Tribolium castaneum. Nature. 2008:452:949-55.

82. Wang L, Wang S, Li Y, Paradesi MSR, Brown SJ. BeetleBase: the model organism database for Tribolium castaneum. Nucleic Acids Res. 2007;35:D476-9.

83. Kim HS, Murphy T, Xia J, Caragea D, Park Y, Beeman RW, et al. BeetleBase in 2010: revisions to provide comprehensive genomic information for Tribolium castaneum. Nucleic Acids Res. 2010;38:D437-42.

84. Bucher G, Scholten J, Klingler M. Parental RNAi in Tribolium (Coleoptera). Curr Biol. 2002;12:R85-6.

85. Tomoyasu Y, Denell RE. Larval RNAi in Tribolium (Coleoptera) for analyzing adult development. Dev Genes Evol. 2004;214:575-8.

86. Lorenzen MD, Berghammer, Brown SJ, Denell RE, Klingler M, Beeman RW. piggyBac-mediated germline transformation in the beetle Tribolium castaneum. Insect Mol Biol. 2003;12:433-40.

87. Schinko JB, Weber M, Viktorinova I, Kiupakis A, Averof M, Klingler M, et al. Functionality of the GAL4/UAS system in Tribolium requires the use of endogenous core promoters. BMC Dev Biol. 2010;10:53.

88. Schinko JB, Hillebrand K, Bucher G. Heat shock-mediated misexpression of genes in the beetle Tribolium castaneum. Dev Genes Evol. 2012;222: $287-98$

89. Dippel S, Oberhofer G, Kahnt J, Gerischer L, Opitz L, Schachtner J, et al. Tissue-specific transcriptomics, chromosomal localization, and phylogeny of chemosensory and odorant binding proteins from the red flour beetle Tribolium castaneum reveal subgroup specificities for olfaction or more general functions. BMC Genomics. 2014;15:1141.

90. Roth LM, Willis ER. Hygroreceptors in adults of Tribolium (Coleoptera, Tenebrionidae). J Exp Zool. 1951:116:527-70.

91. Vogt RG, Riddiford LM. Pheromone binding and inactivation by moth antennae. Nature. 1981;293:161-3.

92. Keil TA. Fine structure of the pheromone-sensitive sensilla on the antenna of the hawkmoth, Manduca sexta. Tissue Cell. 1989;21:139-51.

93. Almaas TJ, Mustaparta H. Pheromone reception in tobacco budworm moth, Heliothis virescens. J Chem Ecol. 1990;16:1331-47.

94. Hill SR, Hansson BS, Ignell R. Characterization of antennal trichoid sensilla from female southern house mosquito, Culex quinquefasciatus Say. Chem Senses. 2009;34:231-52.

95. Alabi T, Marion-Poll F, Danho M, Mazzucchelli GD, De Pauw E, Haubruge E, et al. Identification of taste receptors and proteomic characterization of the antenna and legs of Tribolium brevicornis, a stored food product pest. Insect Mol Biol. 2014;23:1-12

96. Harbach RE, Larsen JR. Fine structure of antennal sensilla of the adult mealworm beetle, Tenebrio molitor L. (Coleoptera: Tenebrionidae). Int J Insect Morphol Embryol. 1977;6:41-60.

97. Shanbhag SR, Müller B, Steinbrecht RA. Atlas of olfactory organs of Drosophila melanogaster. 1. types, external organization, innervation and distribution of olfactory sensilla. Int J Insect Morphol Embryol. 1999;28:377-97.

98. Solari P, Corda V, Sollai G, Kreissl S, Galizia CG, Crnjar R. Morphological characterization of the antennal lobes in the Mediterranean fruit fly Ceratitis capitata. J Comp Physiol A Neuroethol Sens Neural Behav Physiol. 2016;202: $131-46$.
99. Kent KS, Harrow ID, Quartararo P. Hildebrand DJG. An accessory olfactory pathway in Lepidoptera: the labial pit organ and its central projections in Manduca sexta and certain other sphinx moths and silk moths. Cell Tissue Res. 1986:245:237-45.

100. Bräunig P, Pflüger $\mathrm{H}-J$, Hustert R. The specificity of central nervous projections of locust mechanoreceptors. J Comp Neurol. 1983;218:197-207.

101. Nishino H, Nishikawa M, Yokohari F, Mizunami M. Dual, multilayered somatosensory maps formed by antennal tactile and contact chemosensory afferents in an insect brain. J Comp Neurol. 2005;493: 291-308.

102. Dreyer D, Vitt H, Dippel S, Goetz B, El Jundi B, Kollmann M, et al. 3D standard brain of the red flour beetle Tribolium castaneum: a tool to study metamorphic development and adult plasticity. Front Syst Neurosci. 2010;4:3

103. Binzer M, Heuer CM, Kollmann M, Kahnt J, Hauser F, Grimmelikhuijzen CJP, et al. Neuropeptidome of Tribolium castaneum antennal lobes and mushroom bodies. J Comp Neurol. 2014;522:337-57.

104. Miyazaki T, Ito K. Neural architecture of the primary gustatory center of Drosophila melanogaster visualized with GAL4 and LexA enhancer-trap systems. J Comp Neurol. 2010;518:4147-81.

105. Farris SM. Tritocerebral tract input to the insect mushroom bodies. Arthropod Struct Dev, 2008:37:492-503.

106. Tanaka NK, Endo K, Ito K. Organization of antennal lobe-associated neurons in adult Drosophila melanogaster brain. J Comp Neurol. 2012;520:4067-130

107. Frambach I, Schürmann FW. Separate distribution of deutocerebral projection neurons in the mushroom bodies of the cricket brain. Acta Biol Hung. 2004:55:21-9.

108. Groh C, Lu Z, Meinertzhagen IA, Rössler W. Age-related plasticity in the synaptic ultrastructure of neurons in the mushroom body calyx of the adult honeybee Apis mellifera. J Comp Neurol. 2012;520:3509-27.

109. Leiss F, Groh C, Butcher NJ, Meinertzhagen IA, Tavosanis G. Synaptic organization in the adult Drosophila mushroom body calyx. J Comp Neurol. 2009;517:808-24

110. Caron SJC, Ruta V, Abbott LF, Axel R. Random convergence of olfactory inputs in the Drosophila mushroom body. Nature. 2013;497:113-7.

111. de Reuille PB, Routier-Kierzkowska A-L, Kierzkowski D, Bassel GW, Schüpbach T, Tauriello G, et al. MorphoGraphX: a platform for quantifying morphogenesis in 4D. eLife. 2015;4, e05864.

112. Foret S, Maleszka R. Function and evolution of a gene family encoding odorant binding-like proteins in a social insect, the honey bee (Apis mellifera). Genome Res. 2006;16:1404-13.

113. Forêt S, Wanner KW, Maleszka R. Chemosensory proteins in the honey bee: insights from the annotated genome, comparative analyses and expressional profiling. Insect Biochem Mol Biol. 2007;37:19-28.

114. Croset V, Rytz R, Cummins SF, Budd A, Brawand D, Kaessmann H, et al. Ancien protostome origin of chemosensory ionotropic glutamate receptors and the evolution of insect taste and olfaction. PLoS Genet. 2010;6, e1001064.

115. Engsontia P, Sanderson AP, Cobb M, Walden KKO, Robertson HM, Brown S. The red flour beetle's large nose: an expanded odorant receptor gene family in Tribolium castaneum. Insect Biochem Mol Biol. 2008;38:387-97.

116. Nichols Z, Vogt RG. The SNMP/CD36 gene family in Diptera, Hymenoptera and Coleoptera: Drosophila melanogaster, D. pseudoobscura, Anopheles gambiae, Aedes aegypti, Apis mellifera, and Tribolium castaneum. Insect Biochem Mol Biol. 2008;38:398-415.

117. Vogt RG, Miller NE, Litvack R, Fandino RA, Sparks J, Staples J, et al. The insect SNMP gene family. Insect Biochem Mol Biol. 2009;39:448-56.

118. GEO accession: http://www.ncbi.nlm.nih.gov/geo/query/acc.cgi?acc=GSE63162.

119. Enjin A, Zaharieva EE, Frank DD, Mansourian S, Suh GSB, Gallio M, et al. Humidity sensing in Drosophila. Curr Biol. 2016;26:1352-8.

120. Zhang YV, Ni J, Montell C. The molecular basis for attractive salt-taste coding in Drosophila. Science. 2013;340:1334-8.

121. Robertson HM, Kent LB. Evolution of the gene lineage encoding the carbon dioxide receptor in insects. J Insect Sci. 2009;9:19.

122. Kent LB, Robertson HM. Evolution of the sugar receptors in insects. BMC Evol Biol. 2009;9:41.

123. Ni L, Bronk P, Chang EC, Lowell AM, Flam JO, Panzano VC, et al. A gustatory receptor paralogue controls rapid warmth avoidance in Drosophila. Nature 2013;500:580-4.

124. Robertson HM, Wanner KW. The chemoreceptor superfamily in the honey bee, Apis mellifera: expansion of the odorant, but not gustatory, receptor family. Genome Res. 2006;16:1395-403. 
125. Wanner KW, Robertson $\mathrm{HM}$. The gustatory receptor family in the silkworm moth Bombyx mori is characterized by a large expansion of a single lineage of putative bitter receptors. Insect Mol Biol. 2008;17:621-9.

126. Jones WD, Cayirlioglu P, Kadow IG, Vosshall LB. Two chemosensory receptors together mediate carbon dioxide detection in Drosophila. Nature. 2007:445:86-90.

127. Kwon JY, Dahanukar A, Weiss LA, Carlson JR. The molecular basis of $\mathrm{CO}_{2}$ reception in Drosophila. Proc Natl Acad Sci. 2007;104:3574-8.

128. Pitts RJ, Rinker DC, Jones PL, Rokas A, Zwiebel LJ. Transcriptome profiling of chemosensory appendages in the malaria vector Anopheles gambiae reveals tissue- and sex-specific signatures of odor coding. BMC Genomics. 2011;12: 271.

129. Lu T, Qiu YT, Wang G, Kwon JY, Rutzler M, Kwon H-W, et al. Odor coding in the maxillary palp of the malaria vector mosquito Anopheles gambiae. Curr Biol. 2007;17:1533-44.

130. Hartl M, Loschek LF, Stephan D, Siju KP, Knappmeyer C, Kadow ICG. A new Prospero and microRNA-279 pathway restricts $\mathrm{CO}_{2}$ receptor neuron formation. J Neurosci. 2011:31:15660-73.

131. Ramaswamy SB. Behavioural responses of Heliothis virescens (Lepidoptera: Noctuidae) to stimulation with sugars. J Insect Physiol. 1987:33:755-60.

132. de Brito Sanchez MG, Giurfa M, de Paula Mota TR, Gauthier M. Electrophysiological and behavioural characterization of gustatory responses to antennal 'bitter' taste in honeybees. Eur J Neurosci. 2005;22:3161-70.

133. Popescu A, Couton L, Almaas T-J, Rospars J-P, Wright GA, Marion-Poll F, et al. Function and central projections of gustatory receptor neurons on the antenna of the noctuid moth Spodoptera littoralis. J Comp Physiol A. 2013; 199:403-16.

134. Dunipace L, Meister S, McNealy C, Amrein H. Spatially restricted expression of candidate taste receptors in the Drosophila gustatory system. Curr Biol. 2001;11:822-35

135. Jacquin-Joly E, Legeai F, Montagné N, Monsempes C, François M-C, Poulain $\mathrm{J}$, et al. Candidate chemosensory genes in female antennae of the noctuid moth Spodoptera littoralis. Int J Biol Sci. 2012;8:1036-50.

136. Andersson MN, Grosse-Wilde E, Keeling Cl, Bengtsson JM, Yuen MM, Li $M$, et al. Antennal transcriptome analysis of the chemosensory gene families in the tree killing bark beetles, Ips typographus and Dendroctonus ponderosae (Coleoptera: Curculionidae: Scolytinae). BMC Genomics. 2013;14:198.

137. Vosshall LB, Stocker RF. Molecular architecture of smell and taste in Drosophila. Annu Rev Neurosci. 2007;30:505-33.

138. Sparks JT, Bohbot JD, Dickens JC. The genetics of chemoreception in the labella and tarsi of Aedes aegypti. Insect Biochem Mol Biol. 2014;48:8-16.

139. Krieger J. A candidate olfactory receptor subtype highly conserved across different insect orders. J Comp Physiol A Neuroethol Sens Neural Behav Physiol. 2003;189:519-26.

140. Vosshall LB, Hansson BS, A unified nomenclature system for the insect olfactory coreceptor. Chem Senses. 2011;36:497-8.

141. Jones WD, Nguyen T-AT, Kloss B, Lee KJ, Vosshall LB. Functional conservation of an insect odorant receptor gene across 250 million years of evolution. Curr Biol. 2005;15:R119-21.

142. Münch D, Galizia CG. DoOR 2.0 - comprehensive mapping of Drosophila melanogaster odorant responses. Sci Rep. 2016;6:21841.

143. Choo Y-M, Pelletier J, Atungulu E, Leal WS. Identification and characterization of an antennae-specific aldehyde oxidase from the navel orangeworm. PLoS One. 2013;8, e67794.

144. Rybczynski R, Vogt RG, Lerner MR. Antennal-specific pheromone-degrading aldehyde oxidases from the moths Antheraea polyphemus and Bombyx mor J Biol Chem. 1990:265:19712-5.

145. Pelletier J, Bozzolan F, Solvar M, François M-C, Jacquin-Joly E, MaïbècheCoisne M. Identification of candidate aldehyde oxidases from the silkworm Bombyx mori potentially involved in antennal pheromone degradation. Gene. 2007:404:31-40.

146. Ishida Y, Leal WS. Chiral discrimination of the Japanese beetle sex pheromone and a behavioral antagonist by a pheromone-degrading enzyme. Proc Natl Acad Sci. 2008;105:9076-80.

147. Vogt RG. Molecular basis of pheromone detection in insects. Compr Insect Physiol Biochem Pharmacol Mol Biol. 2005;3:753-804.

148. Shi H, Pei L, Gu S, Zhu S, Wang Y, Zhang Y, et al. Glutathione Stransferase (GST) genes in the red flour beetle, Tribolium castaneum, and comparative analysis with five additional insects. Genomics. 2012; 100:327-35.
149. Rogers ME, Jani MK, Vogt RG. An olfactory-specific glutathione-S-transferase in the sphinx moth Manduca sexta. J Exp Biol. 1999;202:1625-37.

150. Zhu F, Moural TW, Shah K, Palli SR. Integrated analysis of cytochrome P450 gene superfamily in the red flour beetle, Tribolium castaneum. BMC Genomics. 2013;14:174.

151. Forstner M, Gohl T, Gondesen I, Raming K, Breer H, Krieger J. Differential expression of SNMP-1 and SNMP-2 proteins in pheromone-sensitive hairs of moths. Chem Senses. 2008:33:291-9.

152. Leitch O, Papanicolaou A, Lennard C, Kirkbride KP, Anderson A. Chemosensory genes identified in the antennal transcriptome of the blowfly Calliphora stygic. BMC Genomics. 2015;16:255.

153. Gu X-C, Zhang Y-N, Kang K, Dong S-L, Zhang L-W. Antennal transcriptome analysis of odorant reception genes in the red turpentine beetle (RTB), Dendroctonus valens. PloS One. 2015;10, e0125159.

154. Wang J, Li D-Z, Min S-F, Mi F, Zhou S-S, Wang M-Q. Analysis of chemosensory gene families in the beetle Monochamus alternatus and its parasitoid Dastarcus helophoroides. Comp Biochem Physiol Part D Genomics Proteomics. 2014:11:1-8.

155. Andersson MN, Videvall E, Walden KK, Harris MO, Robertson HM, Löfstedt C. Sexand tissue-specific profiles of chemosensory gene expression in a herbivorous gall-inducing fly (Diptera: Cecidomyiidae). BMC Genomics. 2014;15:501.

156. Syed Z, Leal WS. Maxillary palps are broad spectrum odorant detectors in culex quinquefasciatus. Chem Senses. 2007;32:727-38.

157. Rinker DC, Zhou X, Pitts RJ, Jones PL, Rokas A, Zwiebel $\sqcup$. RNAseg in the mosquito maxillary palp: a little antennal RNA goes a long way. bioRxiv. 2015:16998. http://dx.doi.org/10.1101/016998.

158. Dweck HK, Ebrahim SA, Khallaf MA, Koenig C, Farhan A, Stieber R, et al. Olfactory channels associated with the Drosophila maxillary palp mediate short- and long-range attraction. eLife. 2016;5:e14925.

159. Haverkamp A, Yon F, Keesey IW, Missbach C, Koenig C, Hansson BS, et al. Hawkmoths evaluate scenting flowers with the tip of their proboscis. eLife. 2016;5:e15039.

160. Ghaninia M, Hansson BS, Ignell R. The antennal lobe of the African malaria mosquito, Anopheles gambiae - innervation and three-dimensional reconstruction. Arthropod Struct Dev. 2007;36:23-39.

161. Anton S, van Loon JJA, Meijerink J, Smid HM, Takken W, Rospars J-P. Central projections of olfactory receptor neurons from single antennal and palpa sensilla in mosquitoes. Arthropod Struct Dev. 2003:32:319-27.

162. Ignell R, Dekker T, Ghaninia M, Hansson BS. Neuronal architecture of the mosquito deutocerebrum. J Comp Neurol. 2005;493:207-40.

163. Dahanukar A, Foster K, van der Goes van Naters WM, Carlson JR. A Gr receptor is required for response to the sugar trehalose in taste neurons of Drosophila. Nat Neurosci. 2001;4:1182-6.

164. Vermehren-Schmaedick A, Scudder C, Timmermans W, Morton DB. Drosophila gustatory preference behaviors require the atypical soluble guanylyl cyclases. J Comp Physiol A. 2011;197:717-27.

165. Bohbot J, Pitts RJ, Kwon H-W, Rützler M, Robertson HM, Zwiebel L. Molecular characterization of the Aedes aegypti odorant receptor gene family. Insect Mol Biol. 2007;16:525-37.

166. Kaupp UB. Olfactory signalling in vertebrates and insects: differences and commonalities. Nat Rev Neurosci. 2010;11:188-200.

167. Goldman AL, Van der Goes van Naters W, Lessing D, Warr CG, Carlson JR. Coexpression of two functional odor receptors in one neuron. Neuron. 2005;45:661-6.

168. Good NE. The flour beetles of the Genus Tribolium. United States Department of Agriculture; 1936

169. Ridley AW, Hereward JP, Daglish GJ, Raghu S, Collins PJ, Walter GH. The spatiotemporal dynamics of Tribolium castaneum (Herbst): adult flight and gene flow. Mol Ecol. 2011;20:1635-46.

170. Farhadian SF, Suárez-Fariñas M, Cho CE, Pellegrino M, Vosshall LB. Postfasting olfactory, transcriptional, and feeding responses in Drosophila. Physiol Behav. 2012;105:544-53.

171. Rinker DC, Pitts RJ, Zhou X, Suh E, Rokas A, Zwiebel L. Blood meal-induced changes to antennal transcriptome profiles reveal shifts in odor sensitivities in Anopheles gambiae. Proc Natl Acad Sci. 2013:110:8260-5.

172. Hu J-H, Wang Z-Y, Sun F. Anatomical organization of antennal-lobe glomeruli in males and females of the scarab beetle Holotrichia diomphalia (Coleoptera: Melolonthidae). Arthropod Struct Dev. 2011;40:420-8.

173. Flook PK, Klee S, Rowell CHF. Combined molecular phylogenetic analysis of the Orthoptera (Arthropoda, Insecta) and implications for their higher systematics. Syst Biol. 1999:48:233-53. 


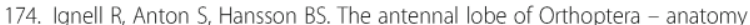
and evolution. Brain Behav Evol. 2001;57:1-17.

175. Farris SM, Roberts NS. Coevolution of generalist feeding ecologies and gyrencephalic mushroom bodies in insects. Proc Natl Acad Sci. 2005;102:17394-9.

176. Kondoh Y, Kaneshiro KY, Kimura K, Yamamoto D. Evolution of sexual dimorphism in the olfactory brain of Hawaiian Drosophila. Proc Biol Sci. 2003;270:1005-13.

177. Kleineidam CJ, Obermayer M, Halbich W, Rössler W. A macroglomerulus in the antennal lobe of leaf-cutting ant workers and its possible functional significance. Chem Senses. 2005;30:383-92.

178. Ågren L. Architecture of a lamellicorn flagellum (Phyllopertha horticola, Scarabaeidae, Coleoptera, Insecta). J Morphol. 1985;186:85-94.

179. Allsopp PG. Sexual dimorphism in the adult antennae of Antitrogus parvulus Britton and Lepidiota negatoria Blackburn (Coleoptera: Scarabaeidae: Melolonthinae). Aust J Entomol. 1990;29:261-6.

180. Ruther J, Reinecke A, Thiemann K, Tolasch T, Francke W, Hilker M. Mate finding in the forest cockchafer, Melolontha hippocastani, mediated by volatiles from plants and females. Physiol Entomol. 2000;25:172-9.

181. Jourdan H, Barbier R, Bernard J, Ferran A. Antennal sensilla and sexual dimorphism of the adult ladybird beetle Semiadalia undecimnotata Schn. (Coleoptera: Coccinellidae). Int J Insect Morphol Embryol. 1995;24: $307-22$.

182. Okada K, Mori M, Shimazaki K, Chuman T. Morphological studies on the antennal sensilla of the cigarette beetle, Lasioderma serricorne ( $F$.) (Coleoptera: Anobiidae). Appl Entomol Zool. 1992;27:269-76.

183. Wu Z, Bin S, He H, Wang Z, Li M, Lin J. Differential expression analysis of chemoreception genes in the striped flea beetle Phyllotreta striolata using a transcriptomic approach. PLoS One. 2016;11, e0153067.

184. Fishilevich E, Vosshall LB. Genetic and functional subdivision of the Drosophila antennal lobe. Curr Biol. 2005;15:1548-53.

185. Grosse-Wilde E, Stieber R, Forstner M, Krieger J, Wicher D, Hansson BS. Sexspecific odorant receptors of the tobacco hornworm Manduca sexta. Front Cell Neurosci. 2010;4:22.

186. Kollmann M, Rupenthal AL, Neumann P, Huetteroth W, Schachtner J. Novel antennal lobe substructures revealed in the small hive beetle Aethina tumida. Cell Tissue Res. 2015;363:679-92.

187. Suzuki T. 4, 8-Dimethyldecanal: the aggregation pheromone of the flour beetles, Tribolium castaneum and T. confusum (Coleoptera: Tenebrionidae). Agric Biol Chem. 1980;44:2519-20,

188. Serrano JM, Castro L, Toro MA, López-Fanjul C. The genetic properties of homosexual copulation behavior in Tribolium castaneum: diallel analysis. Behav Genet. 1991;21:547-58.

189. Posnien N, Koniszewski NDB, Hein HJ, Bucher G. Candidate gene screen in the red flour beetle Tribolium reveals Six3 as ancient regulator of anterior median head and central complex development. PLoS Genet. 2011;7: e1002416.

190. Berghammer, Bucher G, Maderspacher F, Klingler M. A system to efficiently maintain embryonic lethal mutations in the flour beetle Tribolium castaneum. Dev Genes Evol. 1999;209:382-9.

191. Berghammer, Klingler M, Wimmer EA. A universal marker for transgenic insects. Nature. 1999:402:370-1.

192. Horn C, Offen N, Nystedt S, Häcker U, Wimmer EA. PiggyBac-Based insertional mutagenesis and enhancer detection as a tool for functional insect genomics. Genetics. 2003;163:647-61.

193. Horn C, Wimmer EA. A versatile vector set for animal transgenesis. Dev Genes Evol. 2000;210:630-7.

194. Siebert KS, Lorenzen MD, Brown SJ, Park Y, Beeman RW. Tubulin superfamily genes in Tribolium castaneum and the use of a Tubulin promoter to drive transgene expression. Insect Biochem Mol Biol. 2008;38:749-55.

195. Santos JG, Pollák E, Rexer K-H, Molnár L, Wegener C. Morphology and metamorphosis of the peptidergic Va neurons and the median nerve system of the fruit fly, Drosophila melanogaster. Cell Tissue Res. 2006;326: 187-99.

196. Schmitt-Engel C, Schultheis D, Schwirz J, Ströhlein N, Troelenberg N, Majumdar $\mathrm{U}$, et al. The iBeetle large-scale RNAi screen reveals gene functions for insect development and physiology. Nat Commun. 2015;6:7822.

197. Galizia CG, Joerges J, Küttner A, Faber T, Menzel R. A semi-in-vivo preparation for optical recording of the insect brain. J Neurosci Methods. 1997;76:61-9.

198. Pellegrino M, Nakagawa T, Vosshall LB. Single sensillum recordings in the insects Drosophila melanogaster and Anopheles gambiae. J Vis Exp. 2010;36, e1725.
199. Inkscape [Internet]. Available from: http://www.inkscape.org.

200. Huetteroth W, Schachtner J. Standard three-dimensional glomeruli of the Manduca sexta antennal lobe: a tool to study both developmental and adult neuronal plasticity. Cell Tissue Res. 2005;319:513-24.

201. Kurylas AE, Rohlfing T, Krofczik S, Jenett A, Homberg U. Standardized atlas of the brain of the desert locust, Schistocerca gregaria. Cell Tissue Res. 2008; 333:125-45.

202. Brandt R, Rohlfing T, Rybak J, Krofczik S, Maye A, Westerhoff M, et al. Threedimensional averageshape atlas of the honeybee brain and its applications. J. Comp. Neurol. 2005;492:1-19.

203. Schindelin J, Arganda-Carreras I, Frise E, Kaynig V, Longair M, Pietzsch T, et al. Fiji: an open-source platform for biological-image analysis. Nat Methods. 2012;9:676-82.

204. Kent WJ. BLAT_-the BLAST-like alignment tool. Genome Res. 2002:12:656-64.

205. Grabherr MG, Haas BJ, Yassour M, Levin JZ, Thompson DA, Amit I, et al. Fulllength transcriptome assembly from RNA-Seq data without a reference genome. Nat Biotechnol. 2011;29:644-52.

206. Maglott D, Ostell J, Pruitt KD, Tatusova T. Entrez gene: gene-centered information at NCBI. Nucleic Acids Res. 2007:35:D26-31.

207. Pruitt KD, Tatusova T, Maglott DR. NCBI reference sequences (RefSeq): a curated non-redundant sequence database of genomes, transcripts and proteins. Nucleic Acids Res. 2007;35:D61-5.

208. Zdobnov EM, Apweiler R. InterProScan-an integration platform for the signature-recognition methods in InterPro. Bioinformatics. 2001;17:847-8.

209. Attrill H, Falls K, Goodman JL, Millburn GH, Antonazzo G, Rey AJ, et al. FlyBase: establishing a Gene Group resource for Drosophila melanogaster. Nucleic Acids Res. 2016;44:D786-92.

210. Marchler-Bauer A, Lu S, Anderson JB, Chitsaz F, Derbyshire MK, DeWeeseScott C, et al. CDD: a conserved domain database for the functional annotation of proteins. Nucleic Acids Res. 2011;39:D225-9.

211. Petersen TN, Brunak S, von Heijne G, Nielsen H. SignalP 4.0: discriminating signal peptides from transmembrane regions. Nat Methods. 2011;8:785-6.

212. Langmead B, Salzberg SL. Fast gapped-read alignment with Bowtie 2. Nat Methods. 2012;9:357-9.

213. Li H, Handsaker B, Wysoker A, Fennell T, Ruan J, Homer N, et al. The sequence alignment/map format and SAMtools. Bioinformatics. 2009;25:2078-9.

214. Pavlidis $P$, Noble WS. Matrix2png: a utility for visualizing matrix data. Bioinformatics. 2003:19:295-6.

215. R Core Team. R: A Language and Environment for Statistical Computing. 2013. Available from: http://www.R-project.org.

216. Anders S, Huber W. Differential expression analysis for sequence count data. Genome Biol. 2010;11:R106.

217. Gentleman RC, Carey VJ, Bates DM, Bolstad B, Dettling M, Dudoit S, et al. Bioconductor: open software development for computational biology and bioinformatics. Genome Biol. 2004;5:R80.

218. Lawson D, Arensburger $P$, Atkinson P, Besansky NJ, Bruggner RV, Butler R, et al. VectorBase: a data resource for invertebrate vector genomics. Nucleic Acids Res. 2009:37:D583-7.

219. Katoh K, Kuma K, Toh H, Miyata T. MAFFT version 5: improvement in accuracy of multiple sequence alignment. Nucleic Acids Res. 2005;33:511-8.

220. Stamatakis A. RAXML-VI-HPC: maximum likelihood-based phylogenetic analyses with thousands of taxa and mixed models. Bioinformatics. 2006;22: 2688-90.

221. Kapushesky M, Adamusiak T, Burdett T, Culhane A, Farne A, Filippov A, et al. Gene Expression Atlas update-a value-added database of microarray and sequencing-based functional genomics experiments. Nucleic Acids Res. 2011:40:D1077-81.

222. Letunic I, Bork P. Interactive Tree of Life (TOL): an online tool for phylogenetic tree display and annotation. Bioinformatics. 2007;23:127-8.

223. Wang $X$, Fang $X$, Yang $P$, Jiang $X$, Jiang $F$, Zhao $D$, et al. The locust genome provides insight into swarm formation and long-distance flight. Nat Commun. 2014:5:2957.

224. Grabe V, Strutz A, Baschwitz A, Hansson BS, Sachse S. Digital in vivo 3D atlas of the antennal lobe of Drosophila melanogaster. J Comp Neurol. 2015;523: 530-44.

225. Hinke DW. Das relative postembryonale Wachstum der Hirnteile von Culex pipiens, Drosophila melanogaster und Drosophila-mutanten. Z Für Morphol Ökol Tiere. 1961;50:81-118.

226. Qiu YT, van Loon JJA, Takken W, Meijerink J, Smid HM. Olfactory coding in antennal neurons of the malaria mosquito, Anopheles gambiae. Chem Senses. 2006;31:845-63. 
227. Pitts RJ, Zwiebel LJ. Antennal sensilla of two female anopheline sibling species with differing host ranges. Malar J. 2006;5:26.

228. Mclver S. Structure of sensilla trichodea of female Aedes aegypti with comments on innervation of antennal sensilla. J Insect Physiol. 1978:24:383-90.

229. Kent LB, Walden KKO, Robertson HM. The Gr family of candidate gustatory and olfactory receptors in the yellow-fever mosquito Aedes aegypti. Chem Senses. 2008:33:79-93.

230. Koontz MA, Schneider D. Sexual dimorphism in neuronal projections from the antennae of silk moths (Bombyx mori, Antheraea polyphemus) and the gypsy moth (Lymantria dispar). Cell Tissue Res. 1987;249:39-50.

231. Lee JK, Strausfeld NJ. Structure, distribution and number of surface sensilla and their receptor cells on the olfactory appendage of the male moth Manduca sexta. J Neurocytol. 1990;19:519-38.

232. Homberg U, Christensen TA, Hildebrand JG. Structure and function of the deutocerebrum in insects. Annu Rev Entomol. 1989:34:477-501.

233. Kanost MR, Arrese EL, Cao X, Chen Y-R, Chellapilla S, Goldsmith MR, et al. Multifaceted biological insights from a draft genome sequence of the tobacco hornworm moth, Manduca sexta. Insect Biochem Mol Biol. 2016;76:118-47.

234. Rospars JP, Hildebrand JG. Sexually dimorphic and isomorphic glomeruli in the antennal lobes of the Sphinx moth Manduca sexta. Chem Senses. 2000; 25:119-29.

235. Esslen J, Kaissling K-E. Zahl und Verteilung antennaler Sensillen bei der Honigbiene (Apis mellifera L.). Zoomorphologie. 1976;83:227-51.

236. Frasnelli E, Anfora G, Trona F, Tessarolo F, Vallortigara G. Morpho-functional asymmetry of the olfactory receptors of the honeybee (Apis mellifera). Behav Brain Res. 2010;209:221-5.

237. Galizia CG, Sachse S, Rappert A, Menzel R. The glomerular code for odor representation is species specific in the honeybee Apis mellifera. Nat Neurosci. 1999:2:473-8.

238. Mobbs PG. The brain of the honeybee Apis mellifera. I. The connections and spatial organization of the mushroom bodies. Philos Trans R Soc Lond B Biol Sci. 1982;298:309-54

239. Strausfeld NJ. Organization of the honey bee mushroom body: representation of the calyx within the vertical and gamma lobes. J Comp Neurol. 2002:450:4-33.

240. Boeckh J, Ernst K-D. Contribution of single unit analysis in insects to an understanding of olfactory function. J Comp Physiol A. 1987;161:549-65.

241. Neder R. Allometrisches Wachstum von Hirnteilen bei drei verschieden grossen Schabenarten. Zool. Jb., Abt. allg. Zool. u. Physiol. 1959;77:411-464.

242. Greenwood M, Chapman RF. Differences in numbers of sensilla on the antennae of solitarious and gregarious Locusta migratoria L. (Orthoptera: Acrididae). Int J Insect Morphol Embryol. 1984;13:295-301.

243. Laurent G, Naraghi M. Odorant-induced oscillations in the mushroom bodies of the locust. J Neurosci. 1994;14:2993-3004.

244. Altner H. Insektensensillen: Bau und Funktionsprinzipien. Verhandlungen Dtsch Zool Ges. 1977;70:139-153.

Submit your next manuscript to BioMed Central and we will help you at every step:

- We accept pre-submission inquiries

- Our selector tool helps you to find the most relevant journal

- We provide round the clock customer support

- Convenient online submission

- Thorough peer review

- Inclusion in PubMed and all major indexing services

- Maximum visibility for your research

Submit your manuscript at

www.biomedcentral.com/submit

C BioMed Central 


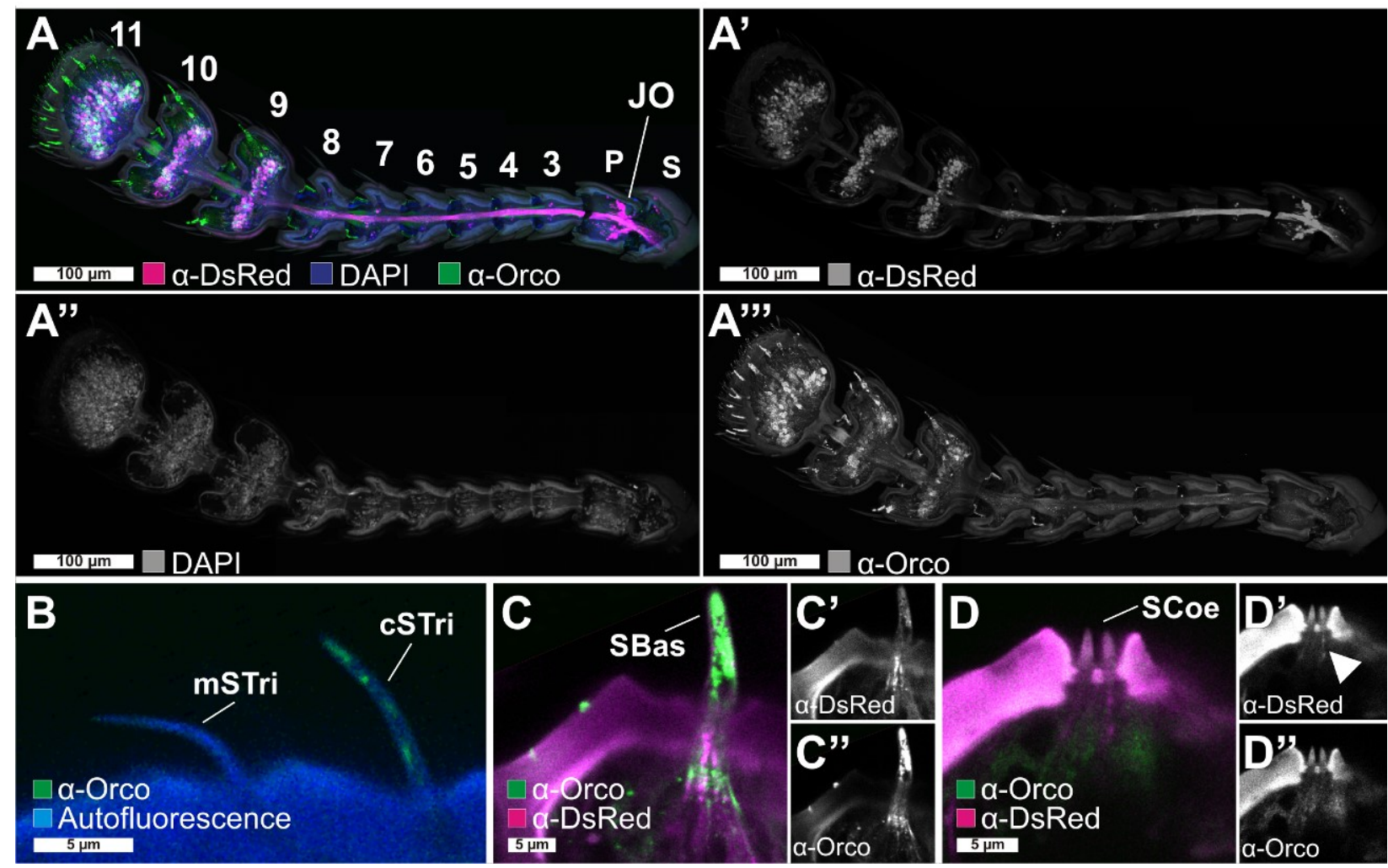

Additional file 1: Figure S1.

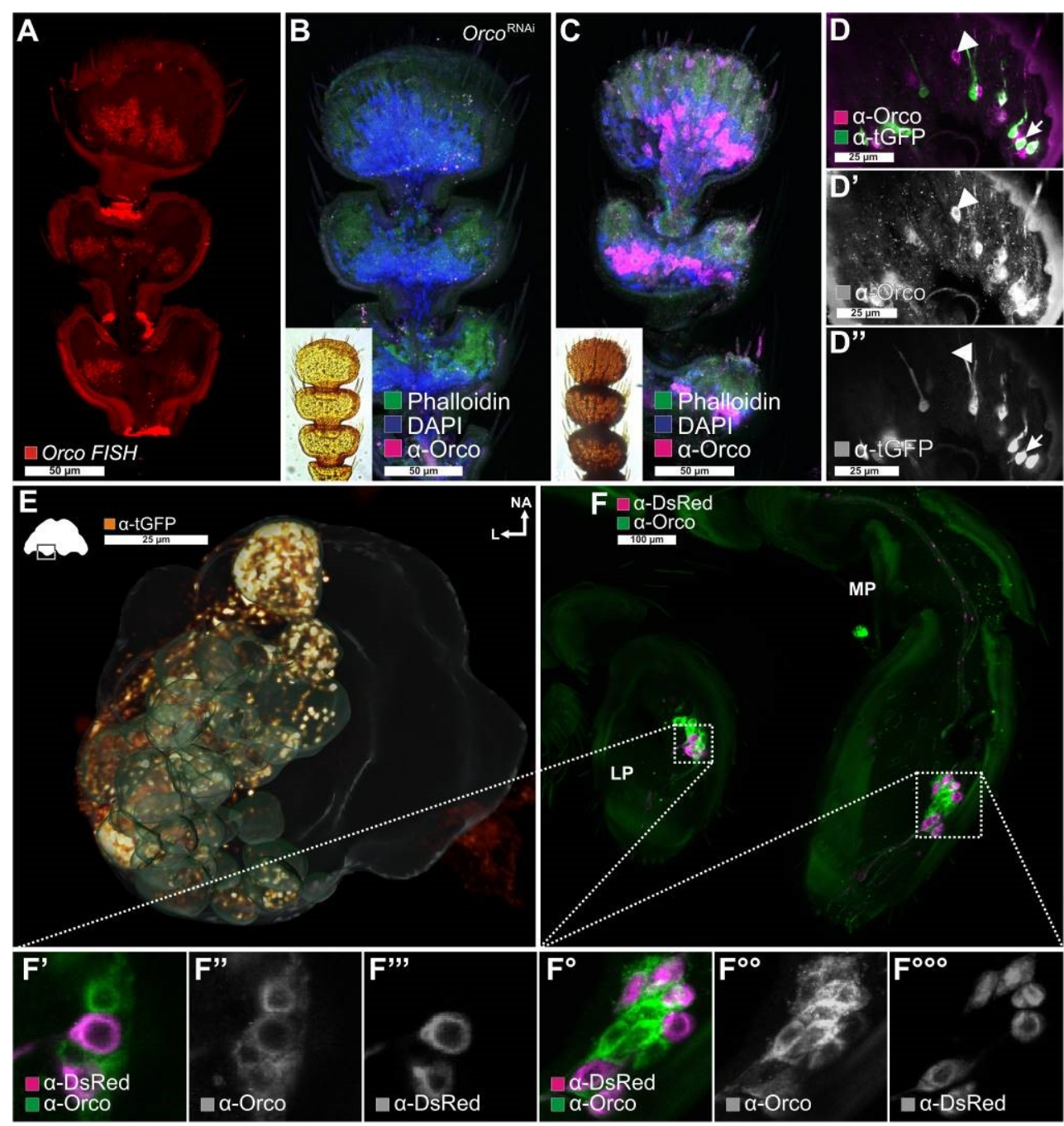

Additional file 2: Figure S2. 

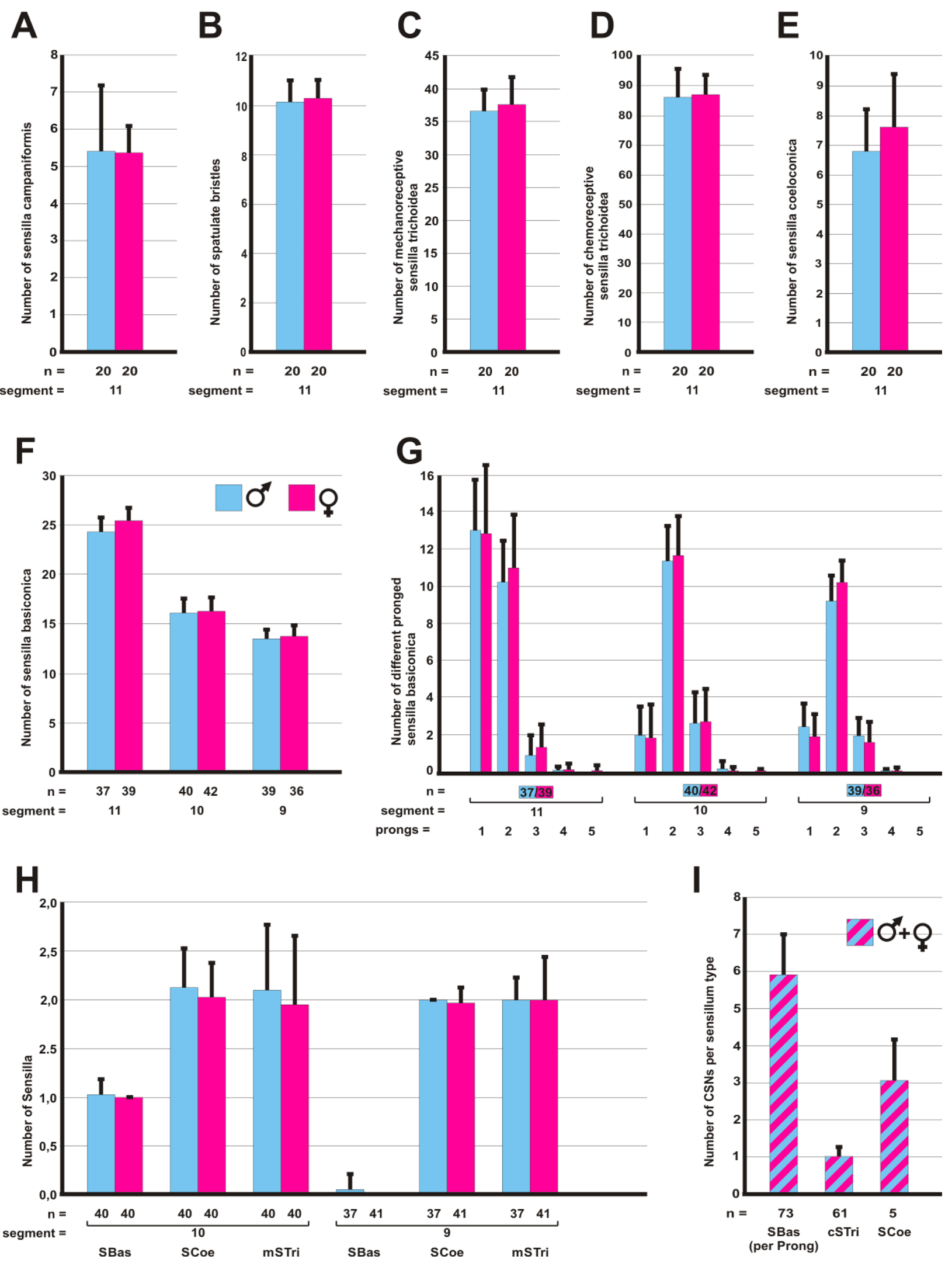

Additional file 3: Figure S3. 


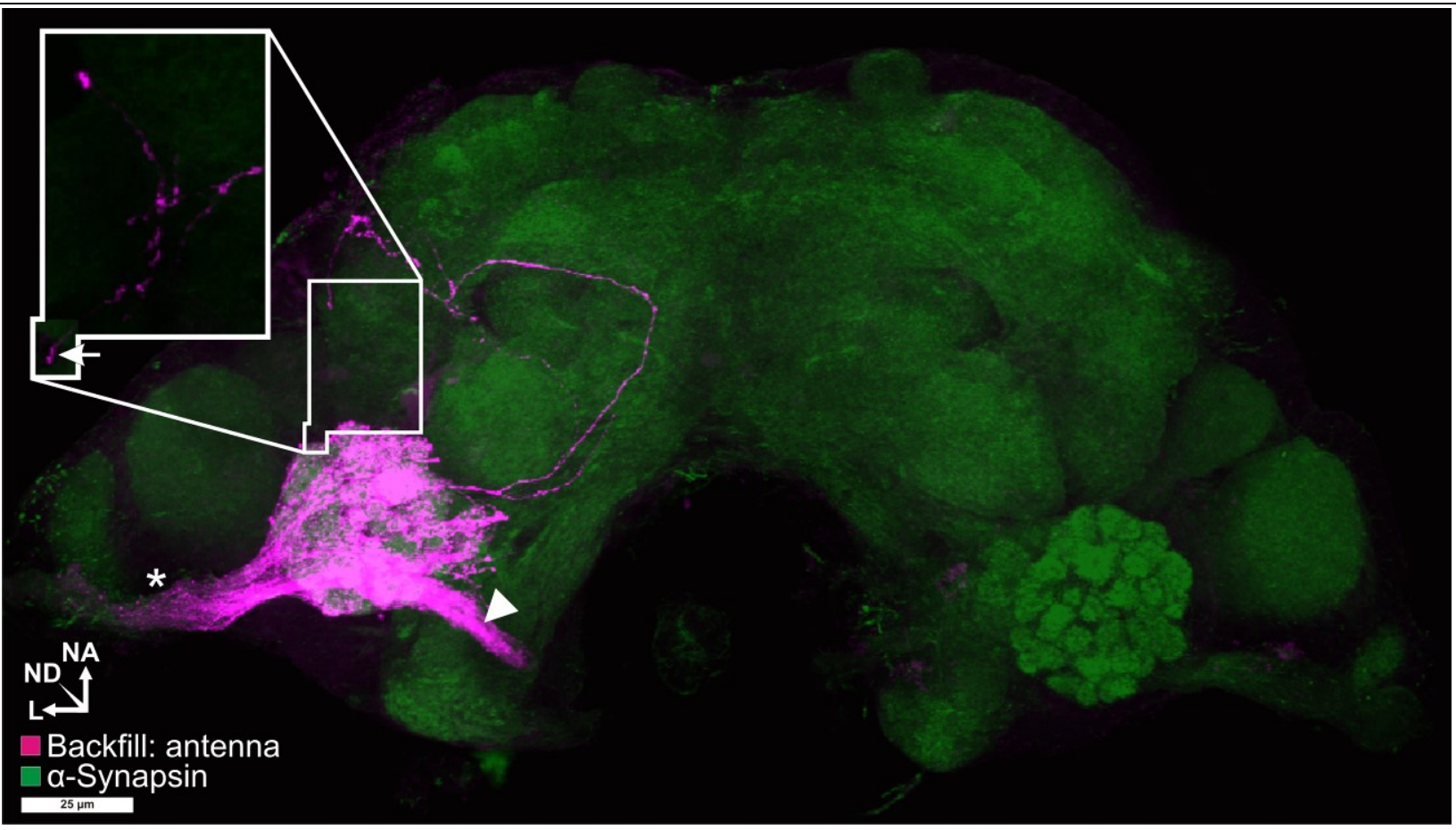

Additional file 6: Figure S4.

A

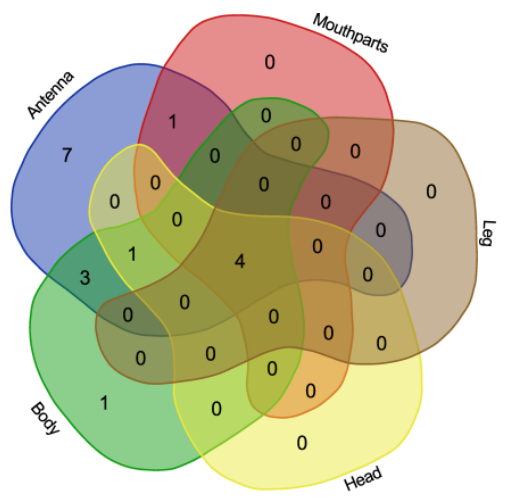

B ChLGX—
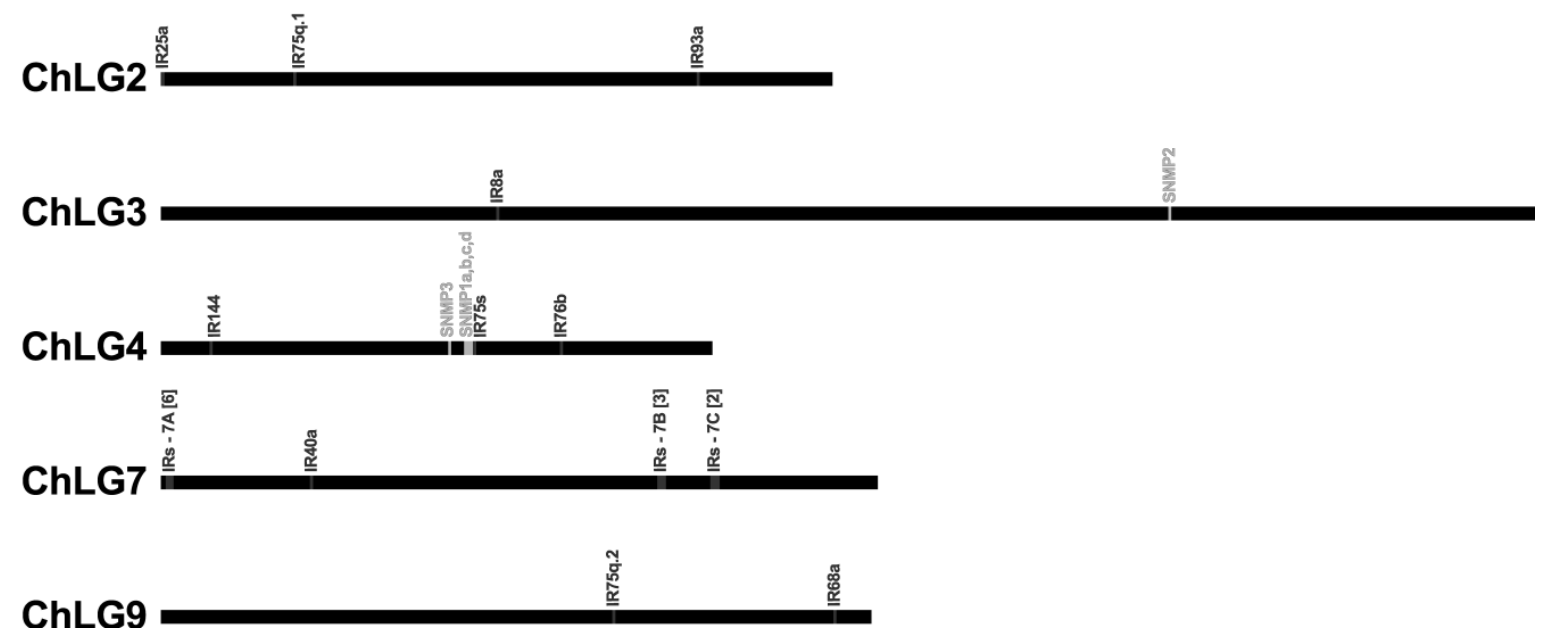

Additional file 9: Figure S5. 


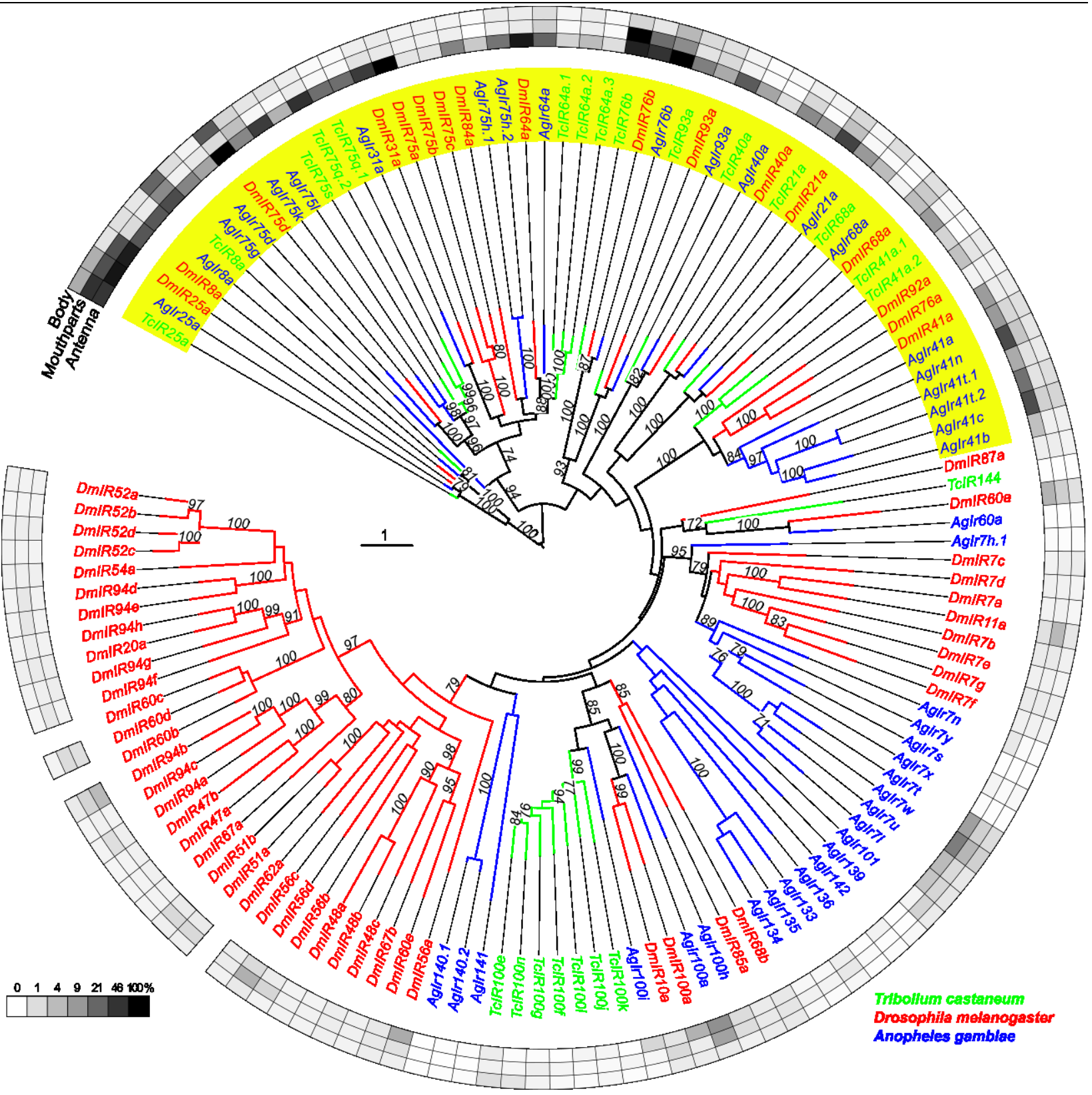

Additional file 10: Figure S6. 
A

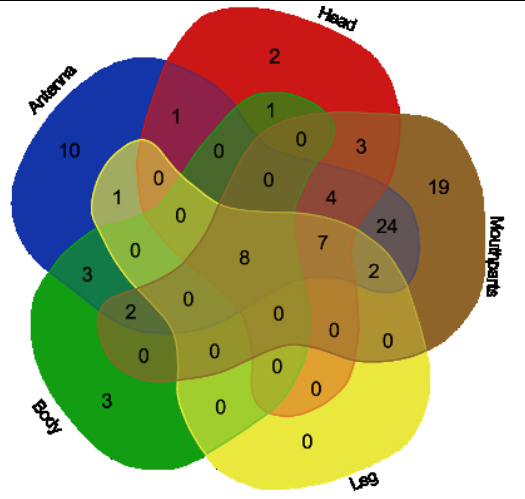

$B_{\text {chLGx }}$
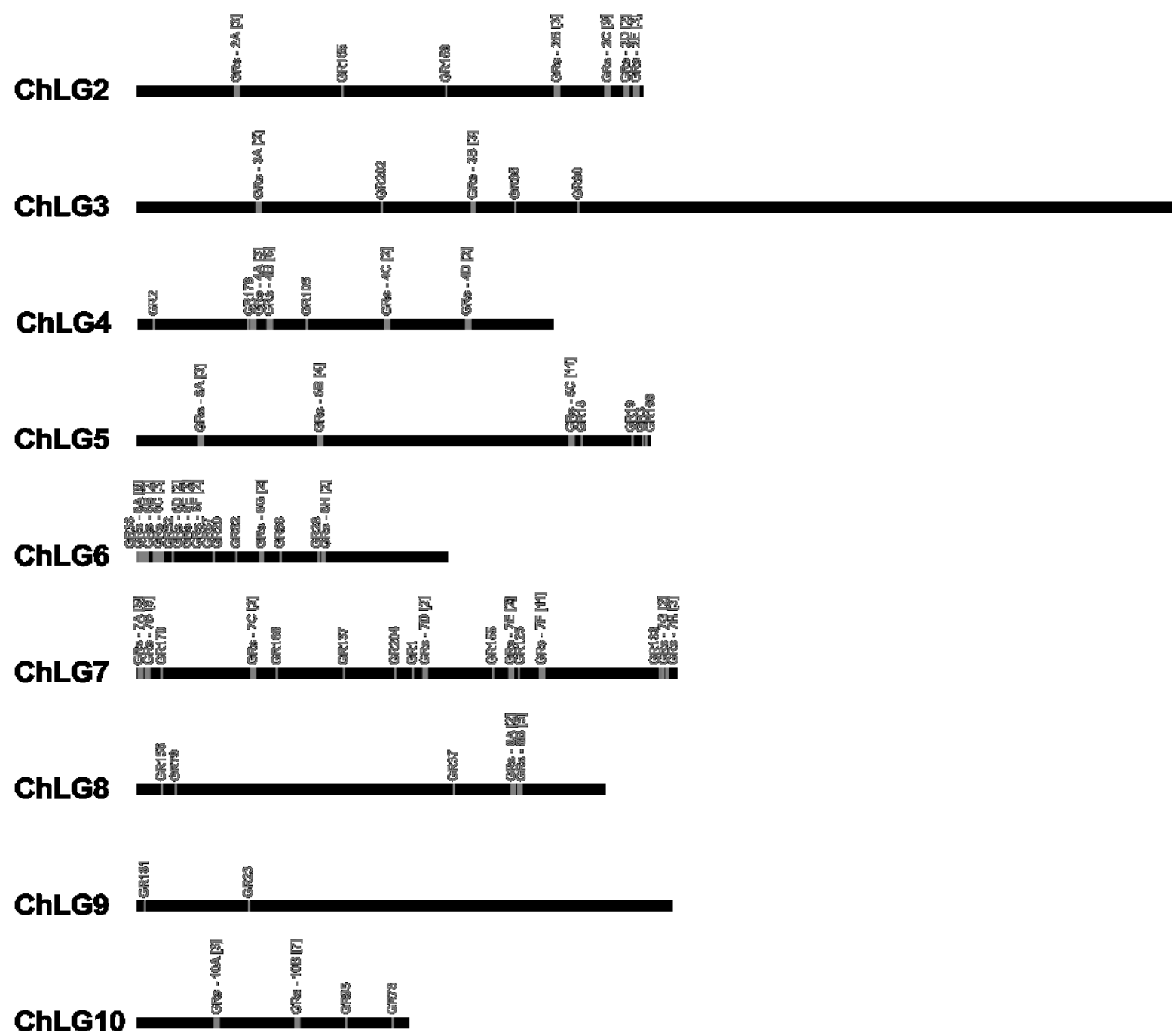

Additional file 11: Figure S7. 


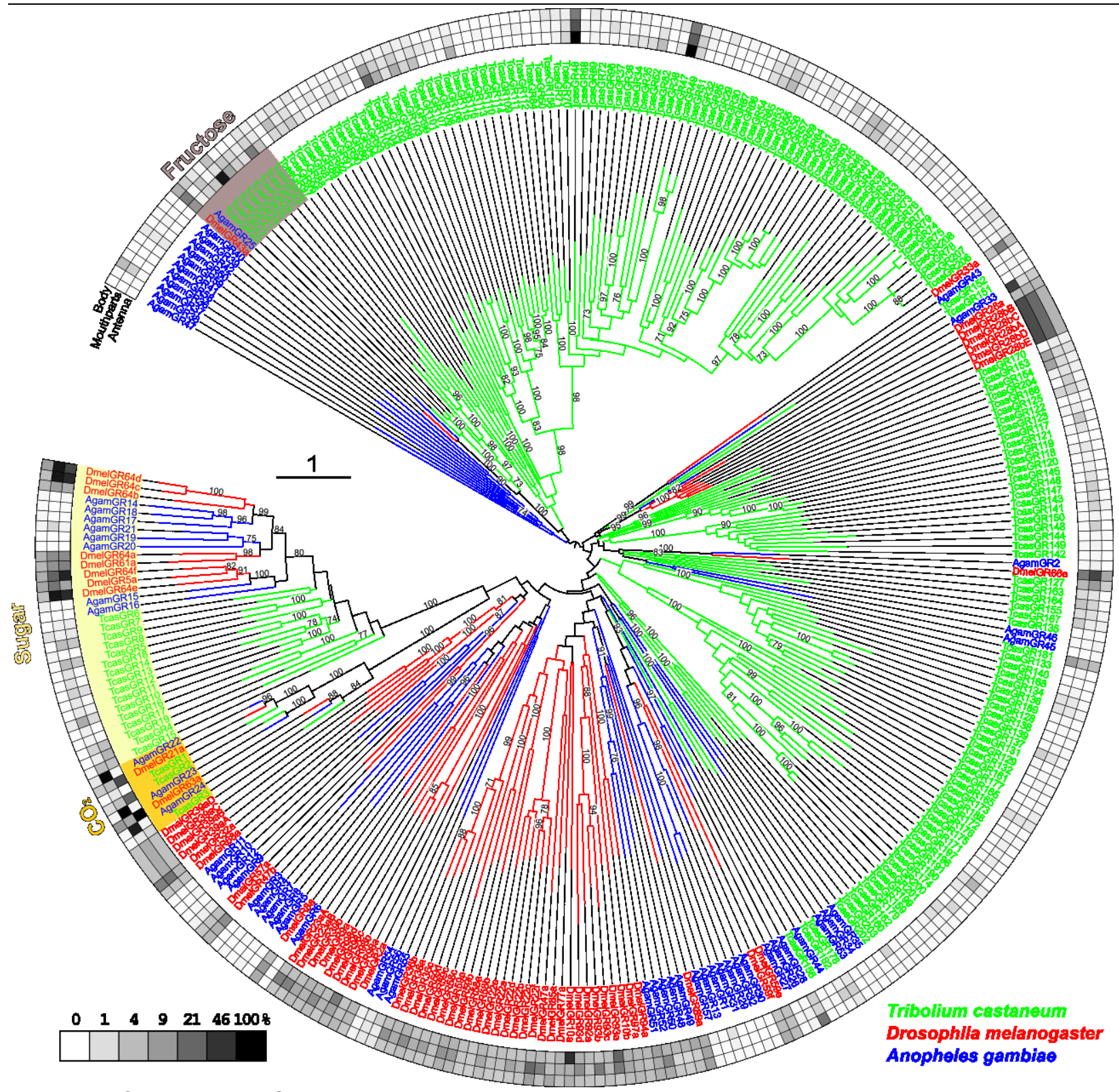

Additional file 12: Figure S8. 

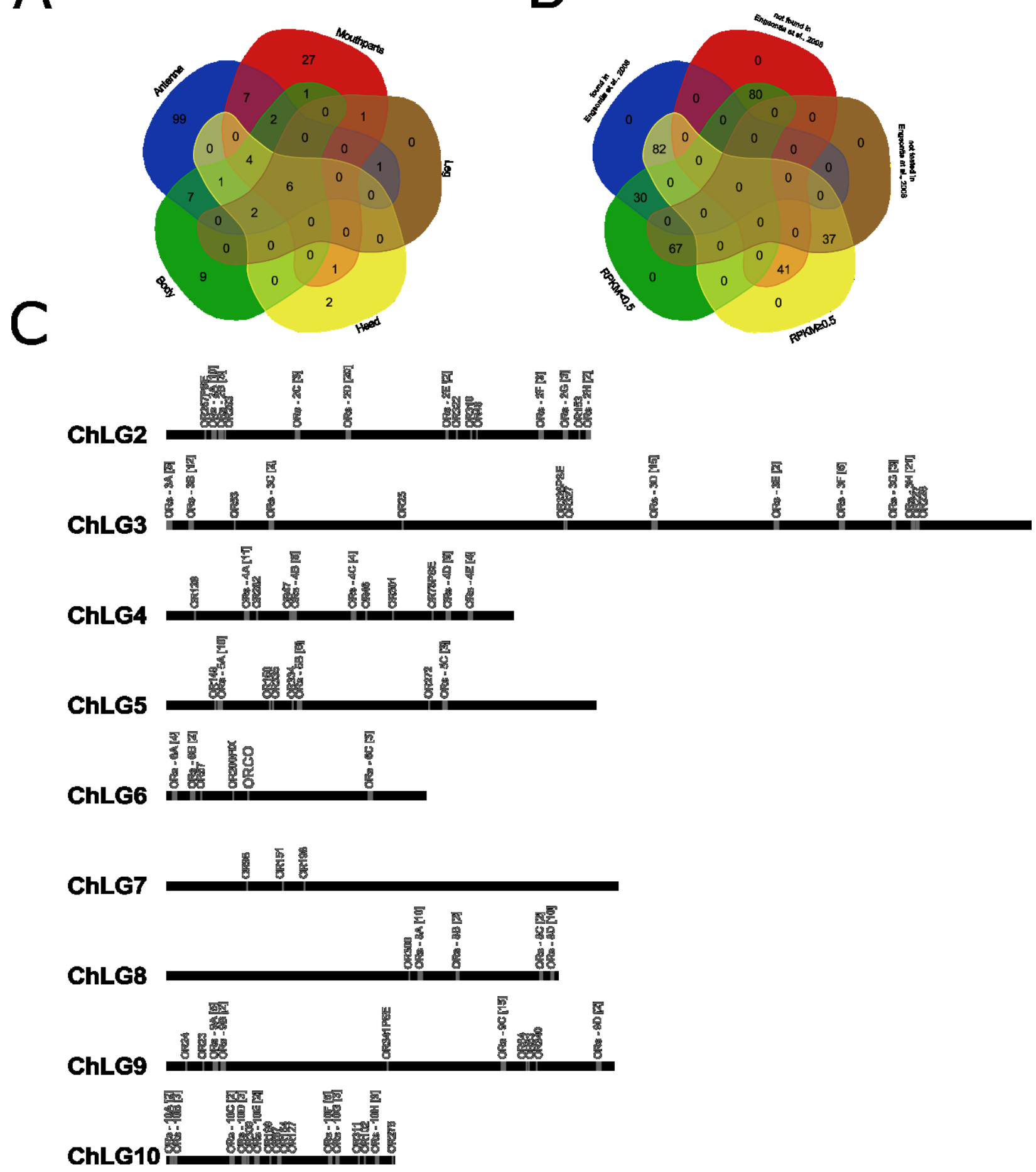

Additional file 13: Figure S9. 


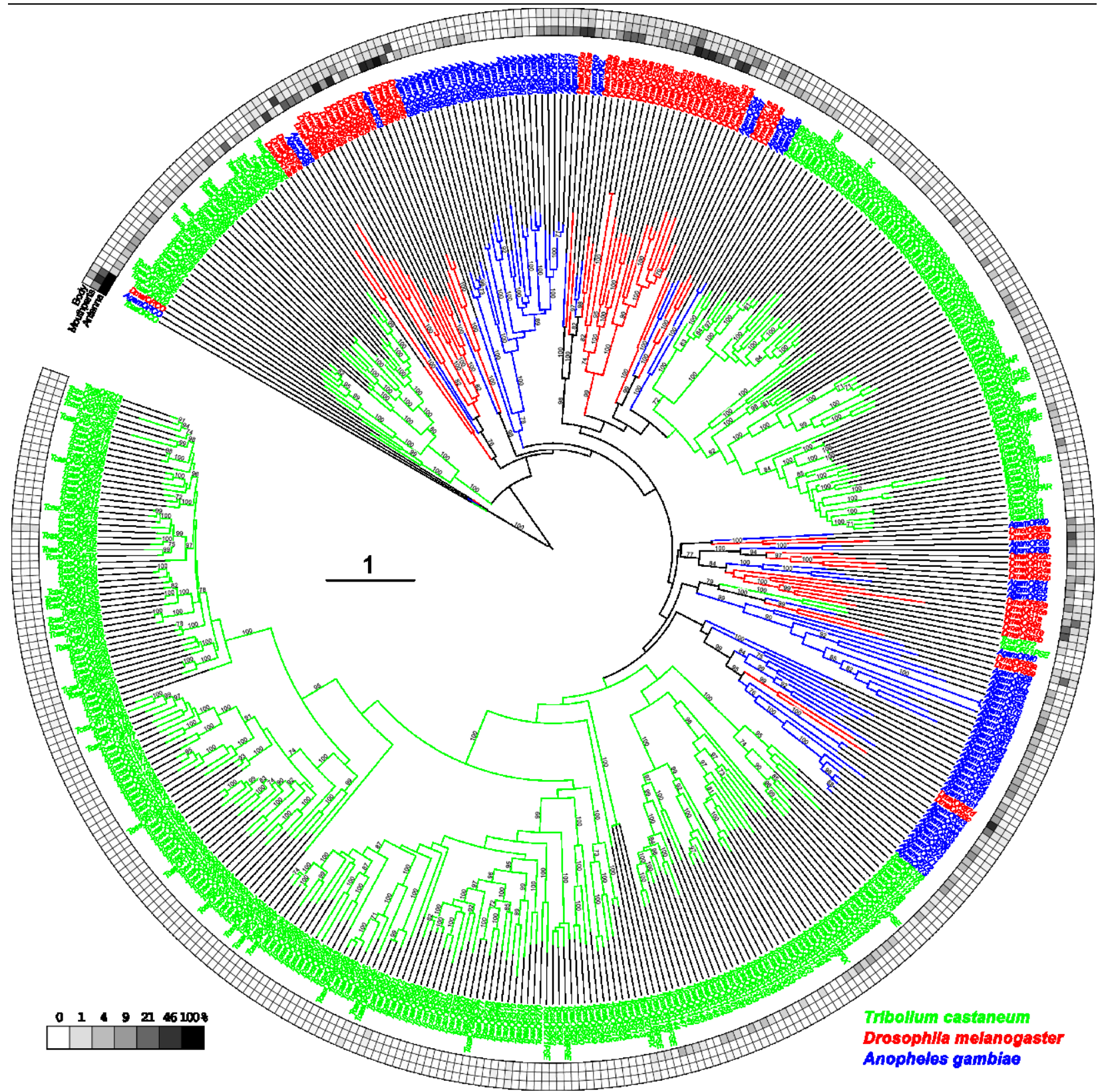

Additional file 14: Figure S10. 

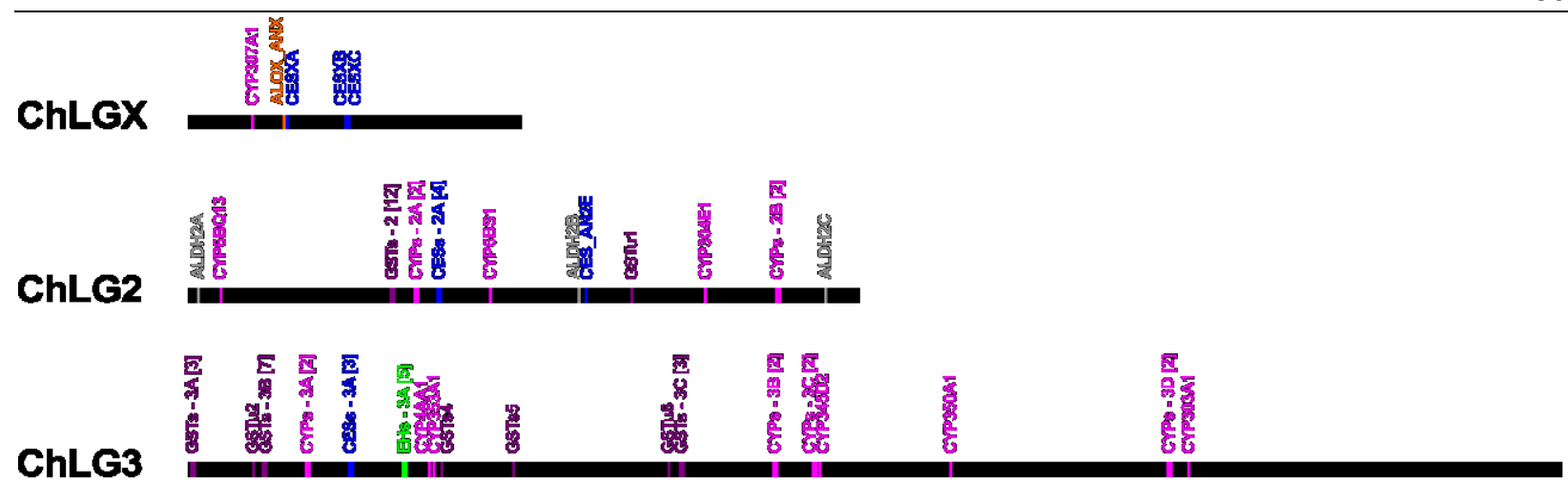

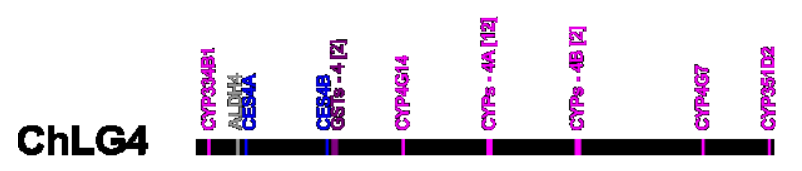
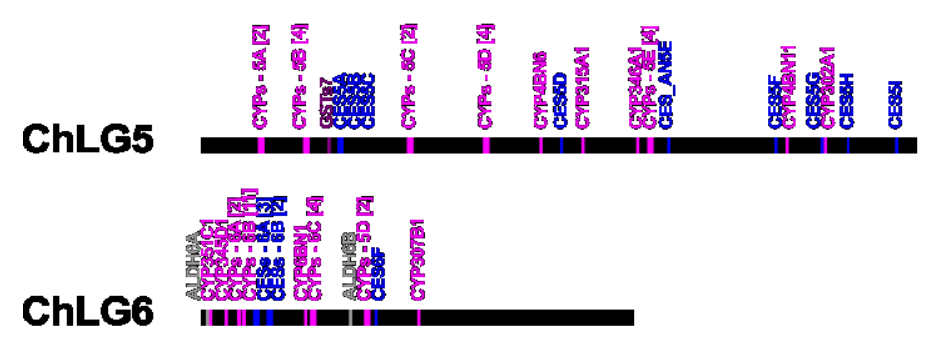

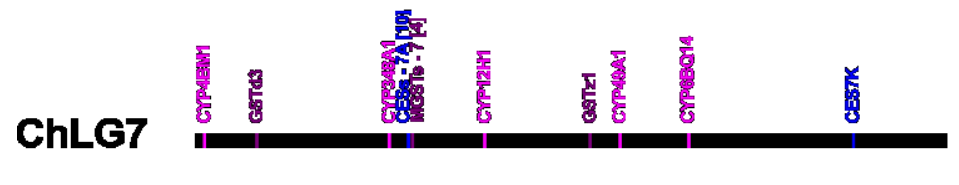
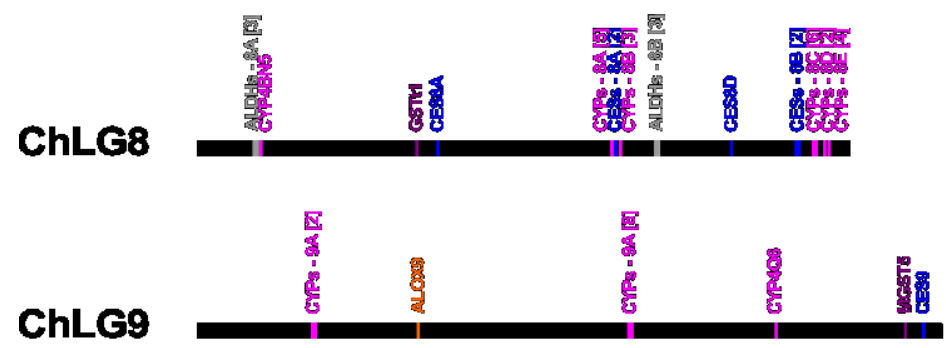

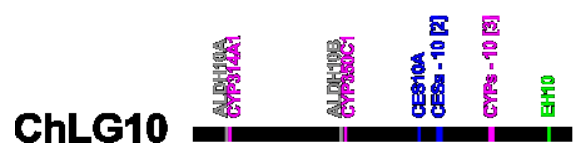

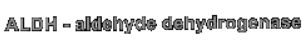

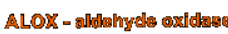

CES - carboxylemtarase

태 - apoxy thydrolab

GST - glutecthlone 8transferase

CYP - cylochrom PASO

Additional file 15: Figure S11. 

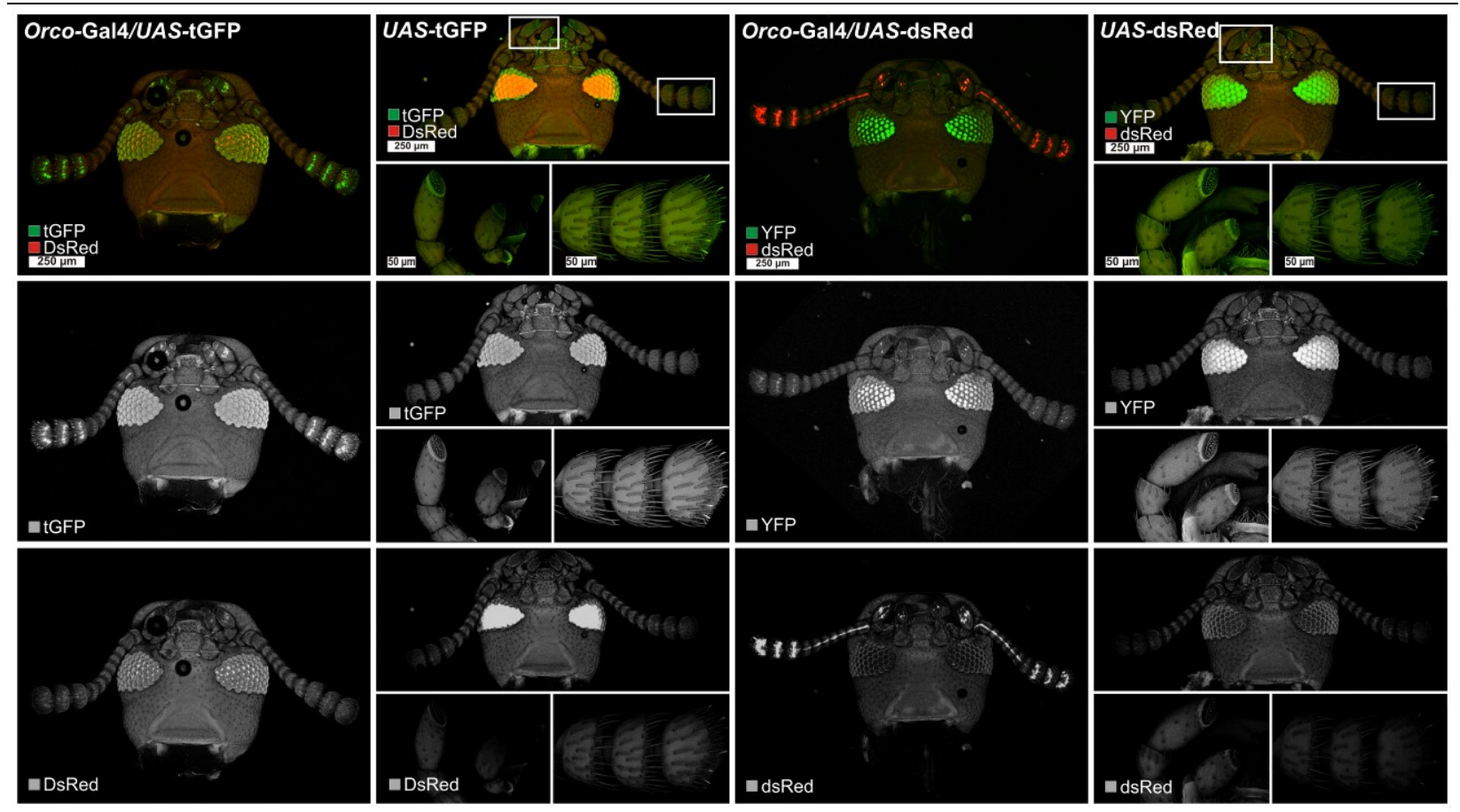

Additional file 17: Figure S12.

\begin{tabular}{|c|c|c|c|c|c|}
\hline \multicolumn{6}{|c|}{ Primary antibodies } \\
\hline Name & Abbreviation & Host Species & Used dilution & Donor and reference / vendor (catalog \#, batch \#, RRID \#) & Specificity \\
\hline D. melanogaster Symapein I & a-Symapsin & Mouse & $1 / 50$ & \begin{tabular}{|l|} 
Dr. E. Buchner, Uriversily of Wurzburg, Germany, Klagges et al., 1996 \\
[245] (n/a, naa, AB_2313617)
\end{tabular} & Utz et al., 2008 [246] \\
\hline Moth Odorant receptor coreceptor & aroreo & Rabbit & $1 / 500$ & Dr. J. Krieger, Martin-Luther-Unwersitat Halo-Wittenbery, Germany & $\begin{array}{l}\text { RNAi Additional File 2 B } \\
\text { and C }\end{array}$ \\
\hline Locusta mlgratorla Tachykhin II & a-TKRP & Rabblt & $1 / 10000$ & 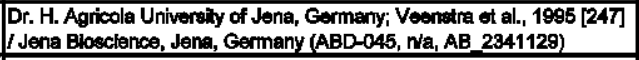 & Binzer ot al, 2014 [103] \\
\hline Red fluorescent protein & o-DsRed & Chicken & $1 / 2000$ & $\begin{array}{l}\begin{array}{l}\text { Rockland Immunochemlcals INC, Limerikk, PA, USA (600-801-379, } \\
26274, \text { AB_10704808) }\end{array} \\
\end{array}$ & \\
\hline Red fluorescent proteln & a-DsRed & Rat & $1 / 1000$ & $\begin{array}{l}\text { ChromoTek GmbH, Planegg-Martineried, Germary (rip-antbody-5f8, } \\
\text { os0428, AB_2336064) }\end{array}$ & \\
\hline Turbo Green fluorescent proteln & $a-t G F$ & Rabblt & $1 / 8000$ & Evrogen, Moscow, Ruseda (AB514, n/a, n/a) & \\
\hline \multicolumn{6}{|c|}{ Secondary antibodies } \\
\hline Name & Abbreviation & Coupled dye & Used dilution & Donor/source, reference & \\
\hline goat ant-rabblt & GAR & Суз & $1 / 300$ & \multicolumn{2}{|c|}{ Jackson ImmunoResearch; Westgrove, PA, USA (111-165-144, N'a, AB_233800e) } \\
\hline goat anti-chicken & GAC & Суз & $1 / 300$ & \multicolumn{2}{|c|}{ Jackson ImmunoResearch; Westgrove, PA, USA (103-165-155, 83117, AB_2337386) } \\
\hline goat enthrabblt & GAR & Cyz & $1 / 300$ & \multicolumn{2}{|c|}{ Jackson ImmunoResearch; Weetgrove, PA, USA (111-225-003, 88408, AB_2307385) } \\
\hline goat anti-rabbit & GAR & Cy5 & $1 / 300$ & \multicolumn{2}{|c|}{ Jackson ImmunoResearch; Westgrove, PA, USA (111-175-144, 76449, AB_2338013) } \\
\hline goat enti-mouse & GAM & Cy5 & $1 / 300$ & \multicolumn{2}{|c|}{ Jackson ImmunoResearch; Wostgrove, PA, USA (115-175-146, 81431, AB_2338713) } \\
\hline goat ant-chlcken & GAC & Alexa488 & $1 / 300$ & \multicolumn{2}{|l|}{ Jackson ImmunoResearch; Westgrove, PA, USA (103-547-008, n/a, n/a) } \\
\hline goat anti-rat & GARat & Cy5 & $1 / 300$ & \multicolumn{2}{|c|}{ Jackson ImmunoResearch; Westgrove, PA, USA (112-175-143, 69074, AB_2336263) } \\
\hline \multicolumn{6}{|c|}{ Dyes and tracers } \\
\hline Name & Abbreviation & stains & Dilution & \multicolumn{2}{|l|}{ Donor / source / reference } \\
\hline 4',6-Diamidin-2-phenylindol & DAPI & muctel & $1 / 20000$ & \multicolumn{2}{|l|}{ StgmaAddrich, Tauniklechen, Germany } \\
\hline Alexa Fuor 488 colplod phalloddln & Phalloddin & $f$-acth & $1 / 200$ & \multirow{2}{*}{\multicolumn{2}{|c|}{ Vector Laboratorles, Burhingame, UK }} \\
\hline Neurobiotin & & neurotracer & $4 \%$ solution & & \\
\hline Texas-Red coupled 3000 MW dextran & & neurotracer & $\begin{array}{l}50 \mathrm{mg} / \mathrm{ml} \text { or } \\
\text { coygtals }\end{array}$ & \multicolumn{2}{|l|}{ Molecular Probes, Invitrogen, Karksuhe, Germany } \\
\hline Bloth coupled $3000 \mathrm{MW}$ dextran & & netrotracer & crystals & \multicolumn{2}{|l|}{ Molecular Probes, mitrogen, Karlsuhe, Germany } \\
\hline Cy3 colpoled Streptandin & & bloth & $1 / 200$ & \multicolumn{2}{|l|}{ Dianova, Hamburg, Germany } \\
\hline
\end{tabular}

\section{References:}

245. Klagges BRE, Heimbeck G, Godenschwege TA, Hofbaver A, Pflugfelder Go, Reifegerste R, et al. Imvertebrate Synapsins: A Single Gene Codes for Several lsoforms in Drosophila. J. Neurosel. 1996;16:3154-65.

246. Utz S, Huetteroth W, Vơmel M, Schachther J. Mas-allatotropin in the developing antennal lobe of the sphinx moth Manduca sexta: Distribution, time course, developmental regulation, and colocallzation wth other neuropeptldes. Dev. Neuroblol. 2008;68:123-42.

247. Veenstra JA, Lau GW, Agrtoola HJ, Petzel DH. Immunohlstologlcal localzation of regulatory peptides in the midgut of the female mosquito Aedes aegypt. Histochern. Cell Blol. 1995;104:337-47.

Additional file 18: Table S2. 


\subsection{Improvement of trangenesis in T. castaneum and establishment of an additional binary expression system (work in progress).}

\section{Introduction}

The success of Drosophila melanogaster as model system for insects is mainly based on the plethora of transgenic tools, which enable the visualization and manipulation of basically every aspect of fly biology. This development started in 1982 with the first stable integration of exogenous DNA into the genome of $D$. melanogaster by using the transposable element $P$ (Rubin and Spradling, 1982). Since then the development of paramount methodology - such as the binary Gal4/UAS expression system (Brand and Perrimon, 1993), the usage of the green fluorescent protein (GFP) as an in vivo marker (Yeh et al., 1995), the possibility to image neuronal activity by expressing genetically encoded calcium sensors (Nakai et al., 2001), and diverse optogenetic tools (Riemensperger et al., 2016) turned D. melanogaster into the prime insect model for neurobiology.

Also the success of $T$. castaneum as an emerging model organism was hugely influenced by the possibility to generate transgenic lines (Berghammer et al., 1999b; Trauner et al., 2009), which is reflected by the impact of the partial OrcoGal4 line to the work described in this thesis. In order to further improve Tribolium as a model system and to optimize the work-flow, I started experiments to increase the transformation efficiency of the predominantly used piggyBac system in $T$. castaneum. The piggyBac transposable element was first discovered in Trichoplusia ni (Fraser et al., 1983) and later related elements were found in several eukaryotic genomes (Sarkar et al., 2003). PiggyBac is one of the most versatile transposase available for germline transformation, it can be used in several insect orders (Lorenzen et al., 2003), has a large cargo capacity ( $\mathrm{Li}$ et al., 2011), and the integration and excision is scar less (Cary et al., 1989). Moreover, in order to compare the expression of more than one gene within the same animal, a second independent binary expression system besides the already established Gal4/UAS system (Schinko et al., 2010) was required. For this aspect the Tet-OFF system seemed suitable (Bello et al., 1998). Since the Tet-OFF system is repressible by tetracycline, its use would also be interesting for the development of modern transgene-based insect pest management strategies (Horn and Wimmer, 2003) in coleopteran pest species.. The Tet-OFF system was developed by the fusion of the tetracycline repressor from E. coli with the VP16 activation domain from the herpes simplex virus (Gossen and Bujard, 1992). In the absence of Tetracycline or its analogs, the tTA protein binds the tetracycline response element (TRE) upstream of a minimal promoter (in case of T. castaenum the hsp68 basal promoter (Schinko et al., 2010) and initiates gene expression. 


\section{Material \& Methods}

\section{Tribolium castaneum rearing}

Tribolium castaneum (Herbst, 1797; Insecta, Coleoptera, Tenebrionidae) strain vermillion ${ }^{\text {white }}$ was bred at about $30^{\circ} \mathrm{C}$ and $40 \%$ relative humidity on organic whole wheat flour supplemented with $5 \%$ yeast powder according to (1999) (Berghammer et al., 1999b).

\section{Embryonic injection}

The embryonic injection was performed in a similar manor as described in (Lorenzen et al., 2003). Eggs were collected at one hour intervals by sieving. After another hour at room temperature, the eggs were washed twice in a $1 \%$ Klorix solution for 30 seconds and aligned on a microscope slide. The injection mix $(0.375 \mu \mathrm{g} / \mu \mathrm{l}$ helper, $0.5 \mu \mathrm{g} / \mu \mathrm{l}$ donor, injection buffer $(1.4 \mathrm{mM} \mathrm{NaCl}$, $0.07 \mathrm{mM} \mathrm{Na}_{2} \mathrm{HPO}_{4}, 0.03 \mathrm{mM} \mathrm{KH} \mathrm{PO}_{4}$, $4 \mathrm{mM} \mathrm{KCl}), 25 \%$ phenol red) was

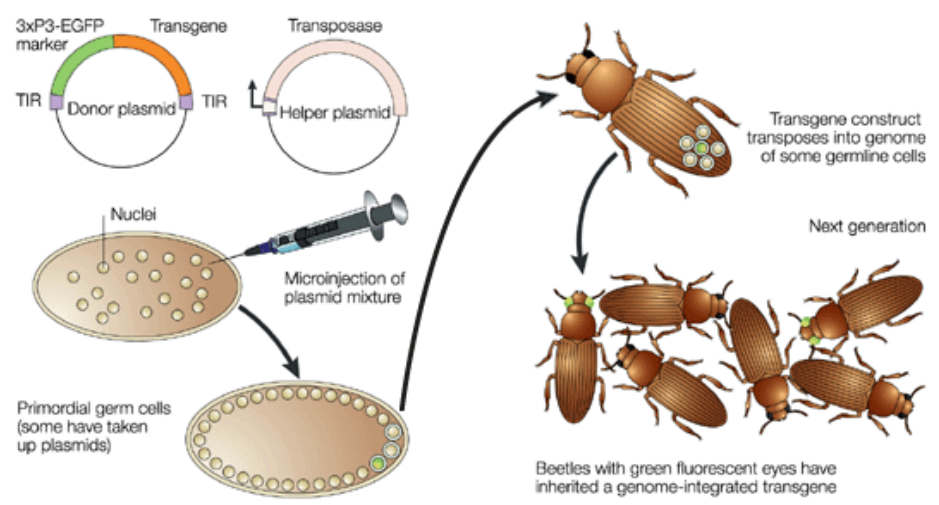

Figure 3.1. Workflow of Tribolium germline transformation. Helper and donor vectors are coinjected in embryos. The transposase expressed form the helper integrates the transgene that is flanked by terminal inverted repeats from the donor vector into the genome of germline cells. Transformed progenies will present the eye-marker.

From Wimmer, 2003 injected into the anterior part of the embryos (Figure 3.1). The eggs were incubated at $30{ }^{\circ} \mathrm{C}$ for about 72 hours in a humid box, followed by 24 hours outside of the box. The hatched larvae were collected and transferred to full grain flour and raised at $30{ }^{\circ} \mathrm{C}$ until pupation. Individual pupae were separated as well as two pupae of the opposite sex from the background strain. The offspring was screened for the eye marker.

\section{Generation of the used plasmids}

The Tribolium specific piggyBac helper Tc_phspBac was generated by cloning the Hpal and Eco72l digested Tribolium hsp68 heat-shock responsive element from pSLfa[Tc'hsp5'-dsRedEx-3'UTR_ 5'3'UTR]fa (Johannes Schinko, formerly AG Bucher, Göttingen) into the EcoRI and Apal digested, blunted and dephosphorylated phspBac (Handler and Harrell li, 1999).

The initial hyperactive piggyBac Tribolium helper TcHyPBase was generated by excision of the HyPBase from PCMV-HyPBase (Yusa et al., 2011) with Kpnl and Xhol and cloning into the same sites in $p S L f a\left[T c^{\prime} h s p 5^{\prime} 3^{\prime} U T R\right] f a$ (Johannes Schinko \#107). The cloning of the later used Versions of the hyperactive helper with mammalian as well as insect optimized codon usage is described in the master thesis of Mohammad KaramiNejadRanjbar. 
The Tet-responder construct $p B a c\left[3 x P 3-d s R e d \_T R E-h s p 68 p\right.$-tGFP-SV40] was generated by cloning an Ascl and HindIII digested PCR product containing the Tchsp68-tGFP-SV40 from pSLfa[UAS-Tc'Hspp-tGFP-SV40]fa (from Johannes Schinko, generated with Ascl-Sacl-hsp68_F ATCGGCGCGCCGAGCTCCGTTTCATATATAAGCGCGGTCTCGCG and pSLfa1180fa_F AACGACGGCCAGTGCC) into a Ascl and Hindlll digested pSLfa1180fa. The TRE was excised with Ascl and Sacl out of the pSL-fa-TRE-hs43-tTA-SV40-fa (Carsten Horn, previously AG Wimmer, Göttingen \#719) and cloned in the same sites into the intermediate $p S L f a\left[T c^{\prime} H s p-p\right.$-tGFP-SV40]. Finally the whole cassette was shuttled by with Ascl into the pBac[3xP3-dsRed]af.

The heat-shock inducible tTA vector pBac[3XP3-eCFP_Tc'hsp5'-tTA-SV40-3'UTR] was generated by cloning a Nhel and Ncol digested PCR product containing the tTA from pSL-fa[TRE-hs43-tTA-SV40]fa (Carsten Horn, previously AG Wimmer, Göttingen \#719; generated with $t T A \_H i n d I I I N h e l f o r$ GCCAAGCTTGCTAGCAAAatgtctagattagataaaagtaagtg and tTA_Ncolrev AgTCCATGGCTACCCACCGTACTC) into the Xbal Ncol digested $p S L f a\left[T c^{\prime} h s p 5^{\prime}-3 ' U T R\right] f a$. After propagation, the $S V 40$ was excised by Ncol out of the ps/1180fa[2.5kb Orcoup-Gal4-SV40] and cloned into the same site. Finally the whole cassette was shuttled by with Ascl and Fsel into the $p B a c[3 x P 3-e C F P] a f$.

The vector maps of the plasmids are available in the appendix.

\section{$\underline{\text { Results }}$}

Evaluation of the piggyBac helper with an endogenous heat-shock responsive element (Tcas_hsp68).

Table 3.1. Comparison of the transformation efficiency of the Drosophila helper ( $p h s p B a c$ ) and the modified version with the Tribolium heat-shock responsive element (Tc_phspBac).

\begin{tabular}{|l|r|r|r|r|r|r|}
\hline donor (about 1,000 embryos each) & \multicolumn{2}{|c|}{ \# single crossings } & \multicolumn{2}{|c|}{ \#vial with transgenic offspring } & \% (\#vials with GMO/\#crossings) \\
\hline & phspBac & Tc_phspBac & phspBac & Tc_phspBac & phspBac & Tc_phspBac \\
\hline pBac[3XP3-dsRed_TRE-tGFP-SV40] & 97 & 102 & 16 & 21 & 16,5 & 20,6 \\
\hline pBac[3XP3-YFP_UAS-dsRed-SV40] & 22 & 27 & 0 & 10 & 0,0 & 37,0 \\
\hline pBac[3XP3-dsRed_UAS-TNXXL-SV40] & 95 & 104 & 6 & 7 & 6,3 & 6,7 \\
\hline pBac[3XP3-CFP_hsp68P-tTA-SV40-3'UTR] & 90 & 77 & 2 & 3 & 2,2 & 3,9 \\
\hline
\end{tabular}

In a first step to improve the transformation efficiency, I used the endogenous heat shock promoter (Schinko et al., 2012) of T. castaneum to drive the transposase. In order to evaluate the differences between the Drosophila helper phspBac (Handler and Harrell li, 1999) and its Tribolium counterpart, I tested them in combination with four different constructs and counted the number of single crossings that gave rise to at least one transgenic beetle. The analysis of the efficiency of the helper constructs revealed a slight increase in the number of injected beetles that gave rise to transgenic offspring (Table 3.1), with one exception 
(pBac[3XP3-YFP_UAS-dsRed-SV40]) (Table 1), which possibly resulted from technical problems.

To further improve the efficiency, I also tested a hyperactive version of the piggyBac transposase (hyPBase) (Yusa et al., 2011). Since it was originally not intended to publish this data, no comparative experiments were performed initially. I simply injected several constructs, to see whether the number of obtained transgenics increased. However, the results seemed extremely promising, with a roughly four-fold increase of

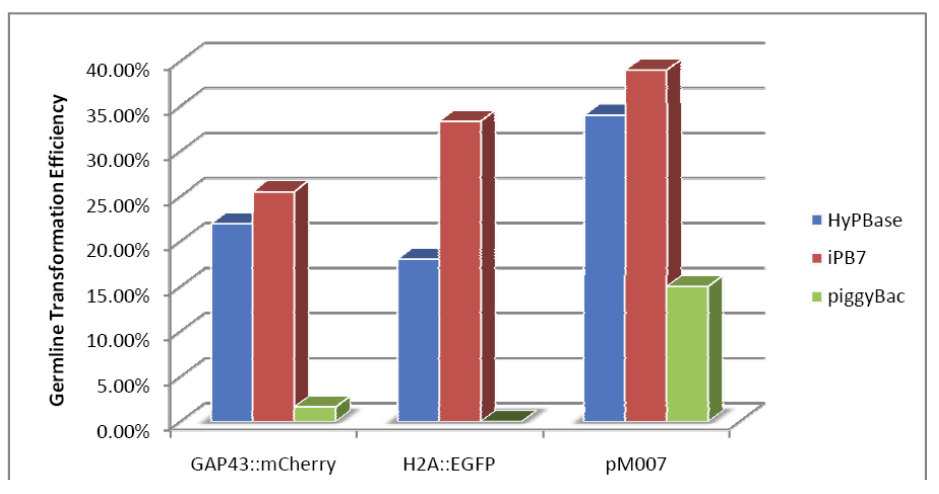

Figure 3.2. Graph representation of germline transfomation efficiencies obtained for different piggyBac transposase variants (from "Germline transformation efficiency of different variants of piggyBac transposase in Tribolium castaneum and Drosophila melanogaster", Master's thesis of Mohammad KaramiNejadRanjbar, 2014) transgenic offspring compared to previous injections with the regular piggyBac transposase. Therefore, besides me also most of my colleagues started to use the improved helper, which became the standard in our laboratory.

After a published study that proposed no increased transformation efficiency of a hyperactive piggyBac in D. melanogaster and Aedes aegypti was published (Wright et al., 2013), we decided to start a systematic approach to do a comparative evaluation of hyperactive piggyBac transposases. The comparison of the wildtype (piggyBac), the mammalian codon optimized (hyPBase), and the insect codon optimized piggyBac transposase (iPB7) was conducted by Mohammad KaramiNejadRanjbar as part of his Master's thesis, which was supervised by me (Figure 3.2). He could conclude: "Hyperactive transposases are highly efficient transgenesis tools in T. castaneum [...] it is clear that hyperactive variants of piggyBac transposase can indeed lead to an up to 20-fold increase in the germline transformation efficiency of $T$. castaneum" (KaramiNejadRanjbar, 2014) dazu muss dann hinten in Verzeichnis Universität, Fakultät Programm!). An independent publication describing the performance of the hyperactive piggyBac transposase in several insects, including besides $T$. castaneum also the Mediterranean fruit fly Ceratitis capitata and the vinegar fly $D$. melanogaster, is currently in preparation with me as co-firstauthor.

\section{The Tet-OFF system in $T$. castaneum}

In oder to evaluate the tet-OFF system in T. castaneum, I crossed the heterozygote heat shock inducible tTA ( $p$ Bac[3XP3-CFP_Tc'hsp5'-tTA-SV40-3'UTR]) line with the heterozygote TREtGFP ( $p B a c\left[3 x P 3-d s R e d \_T R E-h s p 68 p\right.$-tGFP-SV40]) responder line. The obtained offspring 
were sorted based on the eye fluorescence, indicating the presence or absence of the two transgenic constructs into wild type, transactivator only, responder only, and transactivator and responder. The animals were glued to glas slides as indicated in Figure 2 and photographed with a fluorescence stereomicroscope before and two days after the application of a heat shock at $42{ }^{\circ} \mathrm{C}$, for 30 min. Only the pupa containing both, the heat shok inducible tTA and the TRE-tGFP showed a strong reporter expression after the heat shock induction (Figure 3.3). This clearly demonstrates the functionality of the Tet-OFF system as a binary expression system in $T$. castaneum. As the difficulties in cloning intact cis-regulatory regions of $T$. castaneum genes stopped us to generate additional driver lines, this project part was not pursued any

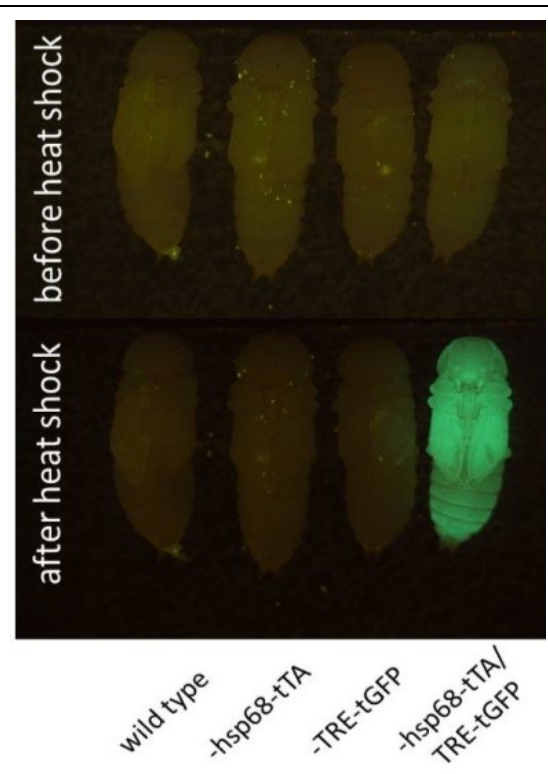

Figure 3.3. Test of the Tet-OFF system in $T$. castaneum. further and tests regarding the represability by tetracycline are still missing.

\section{Discussion}

We could show that in $T$. castaneum the hyperactive piggyBac transposase massively increases the germline transformation rate. Consequently, the number of embryonic microinjection can be significantly reduced from about 1,000 to only 250 embryos for a standard donor construct, to obtain a similar number of transgenic beetles with independent insertions. Not only the reduced number of injections, but subsequently less single crossings and screening effort, lead to a substantial reduction of labor. Additionally, in case of difficult (huge or cytotoxic) donor constructs, the usage of the hyperactive transposase helpers increases the chance to obtain transgenic progeny. Therefore, I strongly recommend the use of the hyperactive piggyBac transposase for $T$. castaenum to optimize work-flow and encourage everyone to test its functionality also in other insect species.

I demonstrated the functionality of the Tet-OFF system as a binary expression system in $T$. castaneum, which can be used in combination with the already established Gal4/UAS system to drive independently reporter or effector genes. The verification of the repressability of the system in $T$. castaneum is still pending. However, having a conditional binary expression systems would enable the adaption of a transgenic pest control system (Horn and Wimmer, 2003; Schetelig and Wimmer, 2011) to this coleopteran species. 


\section{Discussion and Outlook}

\subsection{Involvement of OBPs and CSPs in Olfaction}

We assume an involvement of several OBP and a few CSPs in olfaction based on antennal expression and the presence of a signal peptide for secretion. Furthermore, we could identify several of them at the protein level using MALDI-TOF MS fingerprinting. However, to further substantiate the olfactory function of these candidates additional analysis is required. The expression should be localized on a cellular level by in situ hybridization, as shown for Orco in T. castaneum antennae by Alice Montino (formerly AG Wimmer, Göttingen). Also the localization of the protein should be analyzed by using specific antibodies which should label the sensilla cavity (Steinbrecht et al., 1995), as already shown in preliminary experiments with an antiserum against TcasOBP9B (kindly provided by Paolo Pelosi, Pisa, Italy). This first attempt indicated a localization of TcasOBP9B in the sensilla cavity of several basiconic sensilla (Figure 4.1) and illustrated the possibility of this approach. Additionally, staining the OBPs/CSPs would reveal the number and localization of sensilla that contain a certain OBP/CSP and eventually also the colocalization of different OBPs/CSPs in the same sensillum, as shown in An. gambiae (Schultze et al., 2013). To gain insights into the particular function of these candidates, an RNA interference based loss of function approach, combined with a behavioral (olfactometer) or electrophysiological readout (e.g. electroantennagram)

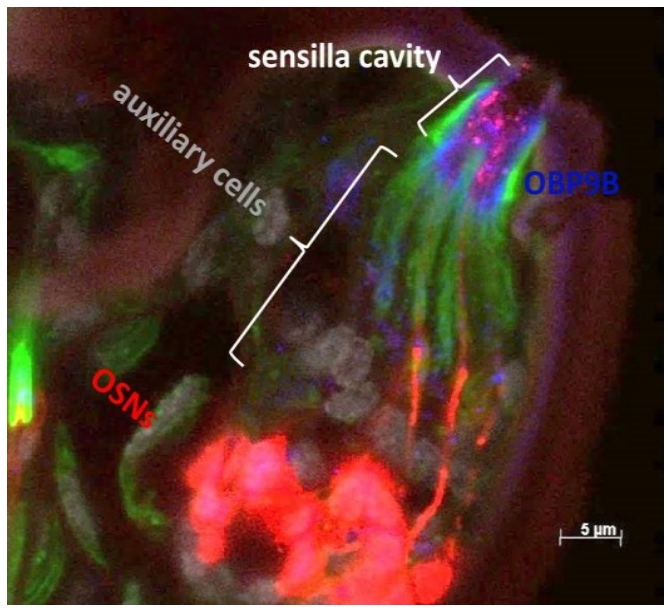

Figure 4.1. IHC staining of a basiconic sensilla in a cryosection of an antenna of the EF1-B-DsRed line, which labels most CSNs, with a polyclonal rabbit antisera against the full length TcasOBP9B (blue), an anti-RFP antibody (red), and DAPI (grey) and phalloidin staining (green). should be performed, as done in $D$. melanogaster (Swarup et al., 2011) or An. gambiae (Pelletier et al., 2010). In cooperation with Prof. Stefan Schütz (Büsgen-Institut, Göttingen) and his $\mathrm{PhD}$ student Karthi Balakrishnan, who performed the EAG measurements, we already started the proposed experiment with promising but very preliminary results. We demonstrated a reduced EAG response towards $\beta$-lonone in TcasOBP9B knockdown beetles (Figure 4.2), which nicely fits to data published on an OBP9B orthologue of a scarab beetle that binds $\beta$-lonone in vitro (Wang et al., 2013). However, the expected results are potentially inconclusive and difficult to interpret, since RNAi leads to a reduction, but not necessarily to a complete loss of the target transcript (Pelletier et al., 2010), most OBPs are important but not essential for odor detection in heterologous systems (Leal, 2013), and heterodimerization, possible cooperativity, or redundancy (Qiao et al., 2011; Wang et al., 2013) can compensate or mask effects. Furthermore the detailed analysis of T. castaneum sensilla revealed that basiconic sensilla tend to harbor usually six to 18 OSNs which are in contact with the OBPs in the surrounding sensilla lymph. The possible interactions 
of odors with several (maybe cooperatively acting) OBPs in combination with several ORs in the same sensillum will make it hard to determine specific interactions. Therefore, also competitive binding studies in vitro with recombinant candidates and mixtures of them are necessary (Qiao et al., 2011; Wang et al., 2013) to gain a rudimentary understanding of their biochemical function.

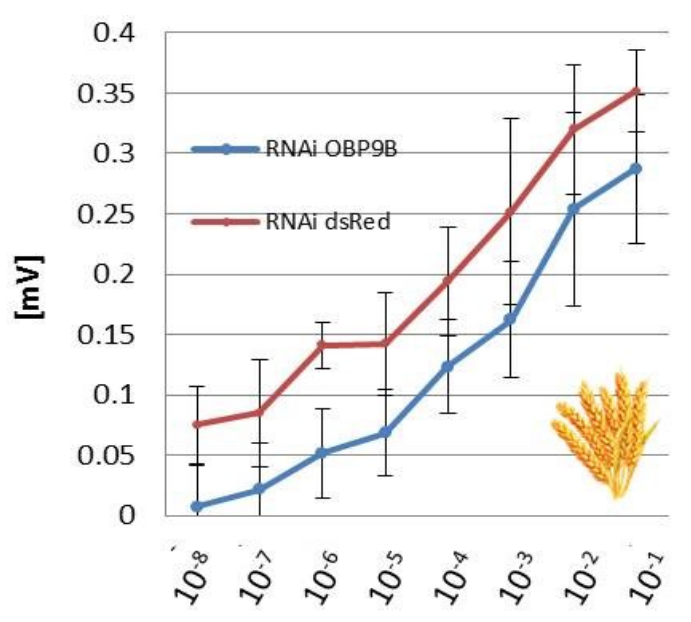

$\beta$-Ionone dilution

Figure 4.2. Figure 1: EAG response to different dilutions of $\beta$-lonone in OBP9B knockdown and control (dsRed) beetles. The measurements were performed with a standard syntech setup; the $\beta$ lonone was diluted in silicone oil; the concentration of the dsRNA of the full length TcasOBP9B and of the mock control (427 bp fragment of dsRed, kindly provided by $\mathrm{G}$. Bucher) was $3 \mu \mathrm{g} / \mu \mathrm{l}$ diluted with injection buffer $(1,4 \mathrm{mM} \mathrm{NaCl}, 0,07 \mathrm{mM}$ $\mathrm{Na} 2 \mathrm{HPO} 4,0,03 \mathrm{mM} \mathrm{KH} 2 \mathrm{PO} 4,4 \mathrm{mM} \mathrm{KCL}$, and $10 \%$ phenol red); the injection was done at about $50 \%$ pupal stage; the beetles were analysed about seven to 14 days after adult eclosion.

\subsection{Deorphanization of IRs, GRs and ORs}

The phylogenetic analysis revealed a substantial radiation of $T$. castaneum GRs and ORs but not of the 'antennal IRs', compared to D. melanogaster and An. gambiae. Most 'antennal IRs', some GRs, but no typical ORs of $T$. castaneum are orthologous to already deorphanized receptors from D. melanogaster (Freeman et al., 2014; Galizia et al., 2010; Jones et al., 2007; Münch and Galizia, 2016; Richards et al., 2008). The large radiation of the identified GRs possibly involved in detection of sweet tastes, makes the ligand prediction questionable. Therefore, the identification of ligands for $T$. castaneum receptors is of interest.

To deorphanize IRs, GRs, or ORs, several methods were developed in the past. For example the ectopic expression and characterization of receptors in a heterologous system, such as cell culture (Kiely et al., 2007) or xenopus oocytes (Wetzel et al., 2001). Both systems are in principle possible, but due to the nature of heterologous systems, results would be always questionable. In all these systems endogenous factors such as OBPs, ODEs, possible coreceptors, and receptor downstream-targets are missing, or have to be supplemented, which massively increases the effort. On the other hand every system brings its own set of proteins, such as channels and kinases, which can interfere with the ectopic receptors and alter their response profiles. Therefore and because of the needed laboraty equipment, I would only use these techniques to verify already obtained results, but not for 'screening' purposes. An alternative is the ectopic expression of chemoreceptors in Drosophila's 'empty neuron system' (Gonzalez et al., 2016) in combination with single sensillum recordings. Since the recopter is 
expressed within OSNs, this technique would give more reliable results. However, endogenous factors (OBPs and ODEs, SNMPs) are missing or have to be supplemented, which will never perfectly emulate the native situation. Moreover, single sensillum recordings require an appropriate setup as well as preperational skills. Therefore, this approach would also not be my first choice. The established misexpression systems and the possibility to knockdown receptor expression by RNAi make it possible to investigate the receptor function directly in T. castaneum, which would eliminate all concerns over missing factors. Single sensillum recordings are in principle possible in $T$. castaneum, but transgenic lines that label OSN subtypes of interest would be required to selectivly record them. However, basiconic sensilla harbor usually six to 18 OSNs, which make it almost impossible to record exclusively from the one neuron of interest in this sensillum. Also, the possibility of coexpression of more than one OR per OSN would require the RNAi mediated knockdown of the candidate receptor to examine whether the measured response was actually generated by it. A similar approach that only relies on RNAi mediated receptor knockdown in combination with electroantennagram (EAG) would work without the need of transgenic lines and could also reveal some ligands for the candidate receptor, as shown for the OBP9B. However, I believe that this approach would not reveal the full response profile of most receptors, due to the high noise of the EAG sum potentials and the redundancy caused by overlapping response profiles of different receptors.

To deorphanize single receptors in T. castaneum, I would express a genetically encoded calcium sensor, such as gCamp (Nakai et al., 2001) under the same control as the respective receptor of interest using the Gal4/UAS system to in vivo image the OSN activity in response to certain odors within the antenna, as shown for D. melanogaster (Pelz et al., 2006). In my opinion this transgenic strategy is the most promising one, but it has to be supplemented with the knockdown of the receptor of interest, to be able to subtract responses from receptors which are possibly coexpressed within the same neurons. However, the generation of the partial OrcoGal4 driver line and my attempts to do the same with several typical ORs revealed that longer non coding DNA regions of $T$. castaenum are extremely instable in E. coli und almost unclonable. Shorter $(\sim 1 \mathrm{kB})$ upstream regions from several other ORs were easy to clone, but failed to drive Gal4 expression in the OSNs, and even the $2.5 \mathrm{kB}$ upstream region of TcasOrco gave rise only to a partial phenocopy of the Orco pattern. Therefore, I suggest to use the recently in $T$. castaneum established CRISPR/Cas9 system (Gilles et al., 2015) to generate the receptor specific driver lines by knocking-in the Gal4 transactivator at the end of the ORF of the receptor gene. To ensure the nuclear localization of the transactivator, I would add a $2 \mathrm{~A}$-like peptide (Daniels et al., 2014) or an IRES (Carter et al., 2008; Savard et al., 2006) before of the Gal4. To improve the visability of the calcium indicator, I would increase the level by using a 20xUAS, as shown in D. melanogaster (Pfeiffer et al., 2010) and if necessary, a multimeric calcium sensor, analog to regular fluorescent proteins (Shearin et al., 2014). In thev case of impairment of gCamp's green signal by antennal cuticle quenching, also a red version ( $R$ - 
GECO) is available (Zhao et al., 2011) and should be tested. Alternatively, the signal could be imaged through the opened head capsule in the target glomerulus of the AL. In case that the receptor gene was disrupted by the insertion of the transactivator, all these experiments should be performed with heterozygoous driver lines, to retain a functional copy of the receptor of interest. However, the results obtained with homozygous beetles, which are knockouts for the receptor of interest, can be used to supplement the results from RNAi treated beetles to substract the influence of possibly coexpressed receptors.

By using this technique, it should be possible to deorphanize several of the receptors of T. castaneum. I would use the already existing partial Orco-Gal4 line to develop a functional reporter system. In parallel, the CRISPR/Cas9 system can be optimized, by using the Orco locus as a target. Already by analysing the responsiveness of the OSNs, labelled with the Orco driver line, the spectrum of odorants received by $T$. castaneum can be determined, which could help to characterize semiochemicals from the native environment. After such a system is fully established, I would continue with the characterization of the highly conserved 'antennal IRs', which could help to examine, whether the sequence conservation is also reflected by their response spectra in comparison to $D$. melanogaster. This would also allow for more statements about insect IRs in general. Prime IR targets are the coreceptors TcasIR8a and TcasIR25a (Rytz et al., 2013), to get an overview of the responsivness of Tribolium's IRs in general. The homologs of IR93a and IR40a, which are necessary for humidity perception in $D$. melanogasters (Enjin et al., 2016) should be tested on, whether they are responsible for the essential role of the antennae in Tribolium's hygroreception (Roth and Willis, 1951). From the GRs, I would focus first on the highly conserved orthologs of the $\mathrm{CO}_{2}$ receptors (TcasGR1, 2 and 3 ), of which one is not present in $D$. melanogaster and is therefore uncharacterized. This would also be interesting for most insects, which have three $\mathrm{CO}_{2}$ receptors (Robertson and Kent, 2009). Also, the analysis of the eight fructose receptor related GRs of $T$. castaneum, which only have a single ortholog in the investigated dipterans, could help to understand the driving force behind this massive radiation of these GR subtype. A characterization of some of the mouthpart enriched ORs (especially of the clades 4, 5, and 6) could reveale wheather the palpal olfaction is used for long distance orientation or to evaluate food over short distances, for example.

\subsection{The innervation of the $A L$ and possible exceptions from the 'Central dogma'}

Based on the number of antennal expressed ORs (129) and the number of AL glomeruli (90), we assumed that in several cases one glomerulus must get input from more than one OSN subtype. Some examples are known in $D$. melanogaster, either through the coexpression of more than one ORs in the same OSN, or through convergence of two different OSNs into the 
same glomerulus (Goldman et al., 2005; Vosshall and Stocker, 2007). However, due to the obvious discrepancy of OR and glomeruli number, we expect that these events are more frequent in $T$. castaneum. To evaluate whether coexpression of more than one OR per OSNs is causing this discrepancy, double fluorescence in situ hybridization would be sufficient (Goldman et al., 2005), but would require a relatively large number of tested candidates in all possible combinations and is not able to show convergence. The only feasible technique to check for convergence of OSN subtypes within the same glomerulus is transgenic based reporter expression, as shown in D. melanogaster (Couto et al., 2005) and mapping of the innervated glomeruli. As discusse above, I suggest also for this purpose the recently in $T$. castaneum established CRISPR/Cas9 system (Gilles et al., 2015), to label the expressing OSNs and consequently the targeted glomeruli of some OSNs.

I would target highly expressed ORs, which are probably present in many OSNs, to investigate whether converging OSN subtypes exists. To further analyse, whether ORs are coexpressed in the same OSN, I would, in analogy to D. melanogaster (Vosshall and Stocker, 2007), choose some closely related ORs which are localized to the same genomic locus, and therefore maybe sharing the same regulative elements, as discussed for clustered OBPs (Librado and Rozas, 2013). Additionally, I would also target GRs, especially orthologs to the $\mathrm{CO}_{2}$ receptors, to investigate whether the single palpal innervated $\mathrm{AL}$ glomerulus gets input from $\mathrm{CO}_{2}$ sensitive GSNs, as shown in several moth species (Kent et al., 1986) and proposed for some mosquitoes (Anton et al., 2003; Ignell et al., 2005). Finally, it should be tested, whether the AL glomeruli which are not innervated by Orco expressing OSNs, are labeled by IR coreceptors (IR8a and IR25a (Rytz et al., 2013)) expressing OSNs.

\subsection{Environmental or age dependent regulation of OR expression}

The genome of $T$. castaneum encodes for 341 OR genes, of which 270 seem to have an intact ORF. Our expression analysis in combination with already published data (Engsontia et al., 2008) revealed about 206 intact ORs to be expressed, indicating that several ORs are not expressed under diverse laboratory conditions. Since unused genes usually undergo pseudogenization, I assume that theses genes may become active under exceptional circumstances (e.g. during long distance flight migration (Ridley et al., 2011)). To investigate this speculation, RNAseq based genome wide expression analysis of several cohorts that were raised under different (harsh) conditions has to be performed, similar to $D$. melanogaster (Hodges et al., 2014) and An. gambiae (Rinker et al., 2013). Later identified candidate ORs can be labelled genetically, as described earlier and could serve as indicators for studying the stimuli that trigger the expression in more detail. 


\subsection{The gnathal olfactory center and its function}

By analyzing palpal backfills and the partial Orco-Gal4 line we identified a novel olfactory center in the gnathal ganglion of $T$. castaneum. Our data clearly indicate that palpal olfactory input enters this glomerularly organized neuropil, which suggests a similar function to the $A L$ of the cerebral ganglion. However, to be able to better characterize this new structure it is necessary to analyse several other receptor (IR, GR, and IR) driver lines. As we found five IRs and several GRs to be expressed in the mouthparts, it should be assessed, whether IR and GR derived input is also processed in the GOC by analyzing already proposed receptor driver lines.

Besides the input neurons, also the processing network within the GOC has to be characterized. The AL of most insects consists of a mainly GABAergic network of local interneurons (Chou et al., 2010; Schachtner et al., 2005). To evaluate whether this also applies to the GOC, immunohistochemistry against GABA (or Glutamate decarboxylase) should be performed and later staining against choline acetyltransferase, to potentially label cholinergic interneurons (Das et al., 2008), and several neuropeptides (Binzer et al., 2014) should be performed to complete the picture.

Finally, the GOC output neurons, analogous to the AL PNs have to be characterized. As most sensory modalities (Heuer et al., 2012) (mainly olfactory input from the AL (Galizia and Rössler, 2010), but also gustatory information from the GNG (Kirkhart and Scott, 2015; Schröter and Menzel, 2003)) are somehow relayed to the MBs in insects, assays should be conducted to find out whether there are direct projections from the GOC to the MBs by labeling GOC associated neurons, including the ascending tracts. However, dye injections into the GOC, similar to the $\mathrm{AL}$, are extremely difficult to perform, due to the relatively small size of less than $20 \mu \mathrm{m}$ in diameter and the almost inaccessible location in the head capsule. Alternatively, similar results could be obtained by using a ubiquitously expressed photoactivatable fluorescent protein and two-photon microscopy in an ex vivo preparation, as shown for $\mathrm{KCs}$ in D. melanogaster (Pech et al., 2013). In the past, all of my attempts to generate a stable and viable mEOS2 (green to red photo-convertible protein) expressing lines in $T$. castaneum failed most likely due to the cytotoxicity in ubiquitous expression. However, in the meantime a stable and functional PA-GFP (C3paGFP) line was generated (personal communication, Peter Kitzmann, AG Bucher, Göttingen), which could
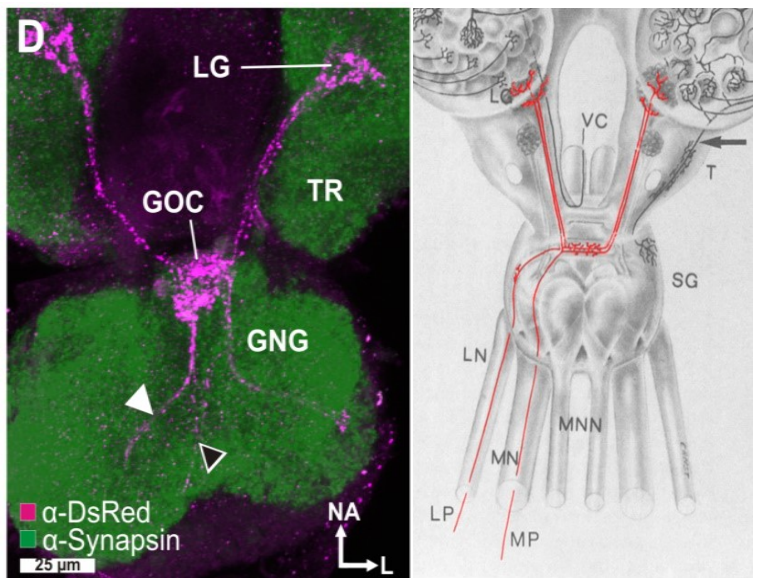

Figure 10. Comparison of the palpal OSNs of $T$. castaneum (partial Orco-Gal4/UAS-dsRed; magenta) and Periplaneta americana (highlighted in red). Modified after Boeckh and Ernst, 1987. be used for this purpose. 
All the supposed techniques can and should be also applied to the lobus glomerulatus, to increase our knowledge of this neuropil, which is of general interest for hemimetabolous insects.

The existence of the GOC in $T$. castaneum was an astonishing observation, which alone justifies the efforts of the past years. To test whether this neuropil is present in other beetles, morphological studies of diverse beetle families should be performed. Furthermore striking similarities of the branching pattern of palpal OSNs of $T$. castaneum and palpal projection in the hemimetabolous cockroach Periplaneta americana (Boeckh and Ernst, 1987) give reason to speculate about the presence of a GOC-like structure in some hemimetabolous insects. To, support this idea, detailed morphological studies on several hemimetabolous orders are required, including palpal backfills in combination with neuropil labeling (e.g. with a synapsin antibody). However, without established transgene based reporter expression systems in a hemimetabolous insect, it is almost impossible to give a solid answer. In my opinion, the most promising hemimetabolous model organism is Gryllus bimaculatus, with available transcriptome data (Zeng et al., 2013), transposon mediated transgenesis (Shinmyo et al., 2004), and established genome editing tools (Awata et al., 2015; Watanabe et al., 2012).

\subsection{Final remarks}

All these findings, especially the identified differences to other species illustrate the spectrum of adaptations that have evolved within the holometabolous insects. This reminds us to avoid over-generalizations on this matter. 


\section{$5 \quad$ References to chapter 2, 3.3, and 4}

Andersson, M.N., Löfstedt, C., and Newcomb, R.D. (2015). Insect olfaction and the evolution of receptor tuning. Chem. Ecol. 3, 53.

Andronopoulou, E., Labropoulou, V., Douris, V., Woods, D.F., Biessmann, H., and latrou, K. (2006). Specific interactions among odorant-binding proteins of the African malaria vector Anopheles gambiae. Insect Mol. Biol. 15, 797-811.

Anton, S., van Loon, J.J.A., Meijerink, J., Smid, H.M., Takken, W., and Rospars, J.-P. (2003). Central projections of olfactory receptor neurons from single antennal and palpal sensilla in mosquitoes. Arthropod Struct. Dev. 32, 319-327.

Arnaud, L., Lognay, G., Verscheure, M., Leenaers, L., Gaspar, C., and Haubruge, E. (2002). Is Dimethyldecanal a Common Aggregation Pheromone of Tribolium Flour Beetles? J. Chem. Ecol. 28, 523-532.

Arnaud, L., Brostaux, Y., Lallemand, S., and Haubruge, E. (2005). Reproductive strategies of Tribolium flour beetles. J. Insect Sci. 5.

Awata, H., Watanabe, T., Hamanaka, Y., Mito, T., Noji, S., and Mizunami, M. (2015). Knockout crickets for the study of learning and memory: Dopamine receptor Dop1 mediates aversive but not appetitive reinforcement in crickets. Sci. Rep. 5, 15885.

Belle, J. de, and Heisenberg, M. (1994). Associative odor learning in Drosophila abolished by chemical ablation of mushroom bodies. Science 263, 692-695.

Bello, B., Resendez-Perez, D., and Gehring, W.J. (1998). Spatial and temporal targeting of gene expression in Drosophila by means of a tetracycline-dependent transactivator system. Development 125 , 2193-2202.

Benelli, G., and Mehlhorn, H. (2016). Declining malaria, rising of dengue and Zika virus: insights for mosquito vector control. Parasitol. Res. 115, 1747-1754.

Benton, R., Sachse, S., Michnick, S.W., and Vosshall, L.B. (2006). Atypical Membrane Topology and Heteromeric Function of Drosophila Odorant Receptors In Vivo. PLOS Biol 4, e20.

Benton, R., Vannice, K.S., Gomez-Diaz, C., and Vosshall, L.B. (2009). Variant lonotropic Glutamate Receptors as Chemosensory Receptors in Drosophila. Cell 136, 149-162.

Berghammer, Bucher, G., Maderspacher, F., and Klingler, M. (1999a). A system to efficiently maintain embryonic lethal mutations in the flour beetle Tribolium castaneum. Dev. Genes Evol. 209, 382-389.

Berghammer, Klingler, M., and Wimmer, E.A. (1999b). A universal marker for transgenic insects. Nature 402, 370-371.

Binzer, M., Heuer, C.M., Kollmann, M., Kahnt, J., Hauser, F., Grimmelikhuijzen, C.J.P., and Schachtner, J. (2014). Neuropeptidome of Tribolium castaneum antennal lobes and mushroom bodies. J. Comp. Neurol. 522, 337-357.

Bloch Qazi, M.C., Boake, C.R.B., and Lewis, S.M. (1998). The femoral setiferous glands of Tribolium castaneum males and production of the pheromone 4,8-dimethyldecanal. Entomol. Exp. Appl. 89, 313317.

Boeckh, J., and Ernst, K.-D. (1987). Contribution of single unit analysis in insects to an understanding of olfactory function. J. Comp. Physiol. A 161, 549-565. 
Brand, A.H., and Perrimon, N. (1993). Targeted gene expression as a means of altering cell fates and generating dominant phenotypes. Dev. Camb. Engl. 118, 401-415.

Briand, L., Swasdipan, N., Nespoulous, C., Bézirard, V., Blon, F., Huet, J.-C., Ebert, P., and Penollet, J.C. (2002). Characterization of a chemosensory protein (ASP3c) from honeybee (Apis mellifera L.) as a brood pheromone carrier. Eur. J. Biochem. FEBS 269, 4586-4596.

Brown, S.J., Shippy, T.D., Miller, S., Bolognesi, R., Beeman, R.W., Lorenzen, M.D., Bucher, G., Wimmer, E.A., and Klingler, M. (2009). The red flour beetle, Tribolium castaneum (Coleoptera): a model for studies of development and pest biology. Cold Spring Harb. Protoc. 2009, pdb.emo126.

Bucher, G., Scholten, J., and Klingler, M. (2002). Parental RNAi in Tribolium (Coleoptera). Curr. Biol. CB 12, R85-86.

Buckland, P.C. (1981). The early dispersal of insect pests of stored products as indicated by archaeological records. J. Stored Prod. Res. 17, 1-12.

Bushey, D., and Cirelli, C. (2011). From genetics to structure to function: exploring sleep in Drosophila. Int. Rev. Neurobiol. 99, 213-244.

Campbell, R.A.A., and Turner, G.C. (2010). The mushroom body. Curr. Biol. 20, R11-R12.

Cao, T.N.P. (2014). Genome annotation and evolution of chemosensory receptors in spider mites. dissertation. Ghent University.

Carter, J.R., Fraser, T.S., and Fraser, M.J. (2008). Examining the relative activity of several dicistrovirus intergenic internal ribosome entry site elements in uninfected insect and mammalian cell lines. J. Gen. Virol. 89, 3150-3155.

Cary, L.C., Goebel, M., Corsaro, B.G., Wang, H.-G., Rosen, E., and Fraser, M.J. (1989). Transposon mutagenesis of baculoviruses: Analysis of Trichoplusia ni transposon IFP2 insertions within the FP-locus of nuclear polyhedrosis viruses. Virology 172, 156-169.

Chapman, A.D. (2009). Numbers of living species in Australia and the world.

Chipman, A.D., Ferrier, D.E.K., Brena, C., Qu, J., Hughes, D.S.T., Schröder, R., Torres-Oliva, M., Znassi, N., Jiang, H., Almeida, F.C., et al. (2014). The First Myriapod Genome Sequence Reveals Conservative Arthropod Gene Content and Genome Organisation in the Centipede Strigamia maritima. PLoS Biol 12, e1002005.

Chou, Y.-H., Spletter, M.L., Yaksi, E., Leong, J.C.S., Wilson, R.I., and Luo, L. (2010). Diversity and wiring variability of olfactory local interneurons in the Drosophila antennal lobe. Nat. Neurosci. 13, 439-449.

Chyb, S. (2004). Drosophila gustatory receptors: from gene identification to functional expression. J. Insect Physiol. 50, 469-477.

Clyne, P.J., Warr, C.G., Freeman, M.R., Lessing, D., Kim, J., and Carlson, J.R. (1999). A novel family of divergent seven-transmembrane proteins: candidate odorant receptors in Drosophila. Neuron 22, 327338.

Couto, A., Alenius, M., and Dickson, B.J. (2005). Molecular, Anatomical, and Functional Organization of the Drosophila Olfactory System. Curr. Biol. 15, 1535-1547.

Croset, V., Rytz, R., Cummins, S.F., Budd, A., Brawand, D., Kaessmann, H., Gibson, T.J., and Benton, R. (2010). Ancient Protostome Origin of Chemosensory lonotropic Glutamate Receptors and the Evolution of Insect Taste and Olfaction. PLoS Genet 6, e1001064. 
Dani, F.R., Michelucci, E., Francese, S., Mastrobuoni, G., Cappellozza, S., Marca, G.L., Niccolini, A., Felicioli, A., Moneti, G., and Pelosi, P. (2011). Odorant-Binding Proteins and Chemosensory Proteins in Pheromone Detection and Release in the Silkmoth Bombyx mori. Chem. Senses 36, 335-344.

Daniels, R.W., Rossano, A.J., Macleod, G.T., and Ganetzky, B. (2014). Expression of Multiple Transgenes from a Single Construct Using Viral 2A Peptides in Drosophila. PLOS ONE 9, e100637.

Das, A., Sen, S., Lichtneckert, R., Okada, R., Ito, K., Rodrigues, V., and Reichert, H. (2008). Drosophila olfactory local interneurons and projection neurons derive from a common neuroblast lineage specified by the empty spiracles gene. Neural Develop. 3, 33.

Depetris-Chauvin, A., Galagovsky, D., and Grosjean, Y. (2015). Chemicals and chemoreceptors: ecologically relevant signals driving behavior in Drosophila. Chem. Ecol. 3, 41.

Dicke, M. (2009). Behavioural and community ecology of plants that cry for help. Plant Cell Environ. 32, 654-665.

Dippel, S., Oberhofer, G., Kahnt, J., Gerischer, L., Opitz, L., Schachtner, J., Stanke, M., Schütz, S., Wimmer, E.A., and Angeli, S. (2014). Tissue-specific transcriptomics, chromosomal localization, and phylogeny of chemosensory and odorant binding proteins from the red flour beetle Tribolium castaneum reveal subgroup specificities for olfaction or more general functions. BMC Genomics 15, 1141.

Doty, R.L. (2015). Handbook of Olfaction and Gustation (John Wiley \& Sons).

Dreyer (2010). 3D standard brain of the red flour beetle Tribolium castaneum: a tool to study metamorphic development and adult plasticity. Front. Syst. Neurosci.

Durand, N., Carot-Sans, G., Bozzolan, F., Rosell, G., Siaussat, D., Debernard, S., Chertemps, T., and Maïbèche-Coisne, M. (2011). Degradation of Pheromone and Plant Volatile Components by a Same Odorant-Degrading Enzyme in the Cotton Leafworm, Spodoptera littoralis. PLoS ONE 6, e29147.

Engsontia, P., Sanderson, A.P., Cobb, M., Walden, K.K.O., Robertson, H.M., and Brown, S. (2008). The red flour beetle's large nose: an expanded odorant receptor gene family in Tribolium castaneum. Insect Biochem. Mol. Biol. 38, 387-397.

Enjin, A., Zaharieva, E.E., Frank, D.D., Mansourian, S., Suh, G.S.B., Gallio, M., and Stensmyr, M.C. (2016). Humidity Sensing in Drosophila. Curr. Biol. 0.

Farhadian, S.F., Suárez-Fariñas, M., Cho, C.E., Pellegrino, M., and Vosshall, L.B. (2012). Post-fasting olfactory, transcriptional, and feeding responses in Drosophila. Physiol. Behav. 105, 544-553.

Farris, S.M., and Van Dyke, J.W. (2015). Evolution and function of the insect mushroom bodies: contributions from comparative and model systems studies. Curr. Opin. Insect Sci. 12, 19-25.

Faustini, D.L., Burkholder, W.E., and Laub, R.J. (1981). Sexually dimorphic setiferous sex patch in the male red flour beetle, Tribolium castaneum (Herbst)(Coleoptera: Tenebrionidae): Site of aggregation pheromone production. J. Chem. Ecol. 7, 465-480.

Faustini, D.L., Rowe, J.R., and Burkholder, W.E. (1982). A male-produced aggregation pheromone in Tribolium brevicornis (LeConte) (Coleoptera: Tenebrionidae) and interspecific responses of several Tribolium species. J. Stored Prod. Res. 18, 153-158.

Fiala, A. (2007). Olfaction and olfactory learning in Drosophila: recent progress. Curr. Opin. Neurobiol. 17, 720-726. 
Fraser, M.J., Smith, G.E., and Summers, M.D. (1983). Acquisition of Host Cell DNA Sequences by Baculoviruses: Relationship Between Host DNA Insertions and FP Mutants of Autographa californica and Galleria mellonella Nuclear Polyhedrosis Viruses. J. Virol. 47, 287-300.

Freeman, E.G., Wisotsky, Z., and Dahanukar, A. (2014). Detection of sweet tastants by a conserved group of insect gustatory receptors. Proc. Natl. Acad. Sci. 201311724.

Friedrich, M., Rambold, I., and Melzer, R.R. (1996). The early stages of ommatidial development in the flour beetle Tribolium castaneum (Coleoptera; Tenebrionidae). Dev. Genes Evol. 206, 136-146.

Galizia, C.G. (2014). Olfactory coding in the insect brain: data and conjectures. Eur. J. Neurosci. 39, 1784-1795.

Galizia, C.G., and Rössler, W. (2010). Parallel Olfactory Systems in Insects: Anatomy and Function. Annu. Rev. Entomol. 55, 399-420.

Galizia, C.G., and Sachse, S. (2010). Odor Coding in Insects. In The Neurobiology of Olfaction, A. Menini, ed. (Boca Raton (FL): CRC Press/Taylor \& Francis), p.

Galizia, C.G., Münch, D., Strauch, M., Nissler, A., and Ma, S. (2010). Integrating Heterogeneous Odor Response Data into a Common Response Model: A DoOR to the Complete Olfactome. Chem. Senses $35,551-563$.

Gilles, A.F., Schinko, J.B., and Averof, M. (2015). Efficient CRISPR-mediated gene targeting and transgene replacement in the beetle Tribolium castaneum. Dev. Camb. Engl. 142, 2832-2839.

Goldman, A.L., Van der Goes van Naters, W., Lessing, D., Warr, C.G., and Carlson, J.R. (2005). Coexpression of Two Functional Odor Receptors in One Neuron. Neuron 45, 661-666.

Gong, D.-P., Zhang, H., Zhao, P., Lin, Y., Xia, Q.-Y., and Xiang, Z.-H. (2007). Identification and expression pattern of the chemosensory protein gene family in the silkworm, Bombyx mori. Insect Biochem. Mol. Biol. 37, 266-277.

Gonzalez, F., Witzgall, P., and Walker, W.B. (2016). Protocol for Heterologous Expression of Insect Odourant Receptors in Drosophila. Chem. Ecol. 24.

Gossen, M., and Bujard, H. (1992). Tight control of gene expression in mammalian cells by tetracyclineresponsive promoters. Proc. Natl. Acad. Sci. 89, 5547-5551.

Grimaldi, D., and Engel, M.S. (2005). Evolution of the Insects (Cambridge University Press).

Handler, A.M., and Harrell Ii, R.A. (1999). Germline transformation of Drosophila melanogaster with the piggyBac transposon vector. Insect Mol. Biol. 8, 449-457.

Hansson, B.S. (2013). Insect Olfaction (Springer Science \& Business Media).

Heuer, C.M., Kollmann, M., Binzer, M., and Schachtner, J. (2012). Neuropeptides in insect mushroom bodies. Arthropod Struct. Dev. 41, 199-226.

Hodges, T.K., Cosme, L.V., Athrey, G., Pathikonda, S., Takken, W., and Slotman, M.A. (2014). Speciesspecific chemosensory gene expression in the olfactory organs of the malaria vector Anopheles gambiae. BMC Genomics 15, 1089.

Horn, C., and Wimmer, E.A. (2003). A transgene-based, embryo-specific lethality system for insect pest management. Nat. Biotechnol. 21, 64-70. 
Howard, R.W. (1987). Chemosystematic Studies of the Triboliini (Coleoptera: Tenebrionidae): Phylogenetic Inferences from the Defensive Chemicals of Eight Tribolium spp., Palorus ratzeburgi (Wissmann), and Latheticus oryzae Waterhouse. Ann. Entomol. Soc. Am. 80, 398-405.

Hu, A., Zhang, W., and Wang, Z. (2010). Functional feedback from mushroom bodies to antennal lobes in the Drosophila olfactory pathway. Proc. Natl. Acad. Sci. 107, 10262-10267.

Ignell, R., Dekker, T., Ghaninia, M., and Hansson, B.S. (2005). Neuronal architecture of the mosquito deutocerebrum. J. Comp. Neurol. 493, 207-240.

Ishida, Y., and Leal, W.S. (2005). Rapid inactivation of a moth pheromone. Proc. Natl. Acad. Sci. U. S. A. 102, 14075-14079.

Ishimoto, H., Takahashi, K., Ueda, R., and Tanimura, T. (2005). G-protein gamma subunit 1 is required for sugar reception in Drosophila. EMBO J. 24, 3259-3265.

Isono, K., and Morita, H. (2010). Molecular and cellular designs of insect taste receptor system. Front. Cell. Neurosci. 4, 20.

Ito, K., Suzuki, K., Estes, P., Ramaswami, M., Yamamoto, D., and Strausfeld, N.J. (1998). The organization of extrinsic neurons and their implications in the functional roles of the mushroom bodies in Drosophila melanogaster Meigen. Learn. Mem. Cold Spring Harb. N 5, 52-77.

Jefferis, G.S.X.E. (2005). Insect Olfaction: A Map of Smell in the Brain. Curr. Biol. 15, R668-R670.

Jefferis, G.S., Marin, E.C., Stocker, R.F., and Luo, L. (2001). Target neuron prespecification in the olfactory map of Drosophila. Nature 414, 204-208.

Jiao, Y., Moon, S.J., Wang, X., Ren, Q., and Montell, C. (2008). Gr64f is required in combination with other gustatory receptors for sugar detection in Drosophila. Curr. Biol. CB 18, 1797-1801.

Jones, W.D., Cayirlioglu, P., Kadow, I.G., and Vosshall, L.B. (2007). Two chemosensory receptors together mediate carbon dioxide detection in Drosophila. Nature 445, 86-90.

KaramiNejadRanjbar, M. (2014). Germline transformation efficiency of different variants of piggyBac transposase in Tribolium castaneum and Drosophila melanogaster. Master's Thesis in Molecular Biology Graduate Program at the Georg August University Göttingen, Faculty of Biology.

Keller, A., and Vosshall, L.B. (2003). Decoding olfaction in Drosophila. Curr. Opin. Neurobiol. 13, 103110.

Kent, K.S., Harrow, I.D., Quartararo, P., and Hildebrand, D.J.G. (1986). An accessory olfactory pathway in Lepidoptera: the labial pit organ and its central projections in Manduca sexta and certain other sphinx moths and silk moths. Cell Tissue Res. 245, 237-245.

Kiely, A., Authier, A., Kralicek, A.V., Warr, C.G., and Newcomb, R.D. (2007). Functional analysis of a Drosophila melanogaster olfactory receptor expressed in Sf9 cells. J. Neurosci. Methods 159, 189-194.

Kim, H.S., Murphy, T., Xia, J., Caragea, D., Park, Y., Beeman, R.W., Lorenzen, M.D., Butcher, S., Manak, J.R., and Brown, S.J. (2010). BeetleBase in 2010: revisions to provide comprehensive genomic information for Tribolium castaneum. Nucleic Acids Res. 38, D437-442.

Kirkhart, C., and Scott, K. (2015). Gustatory learning and processing in the Drosophila mushroom bodies. J. Neurosci. Off. J. Soc. Neurosci. 35, 5950-5958. 
Krieger, J. (2003). A candidate olfactory receptor subtype highly conserved across different insect orders. J. Comp. Physiol. A Neuroethol. Sens. Neural. Behav. Physiol. 189, 519-526.

Kwon, J.Y., Dahanukar, A., Weiss, L.A., and Carlson, J.R. (2007). The molecular basis of CO2 reception in Drosophila. Proc. Natl. Acad. Sci. U. S. A. 104, 3574-3578.

Larsson, M.C., Domingos, A.I., Jones, W.D., Chiappe, M.E., Amrein, H., and Vosshall, L.B. (2004). Or83b encodes a broadly expressed odorant receptor essential for Drosophila olfaction. Neuron 43, 703-714.

Laska, M., Galizia, C.G., Giurfa, M., and Menzel, R. (1999). Olfactory discrimination ability and odor structure-activity relationships in honeybees. Chem. Senses $24,429-438$.

Leal, W.S. (2013). Odorant Reception in Insects: Roles of Receptors, Binding Proteins, and Degrading Enzymes. Annu. Rev. Entomol. 58, 373-391.

Lee, Y., Moon, S.J., and Montell, C. (2009). Multiple gustatory receptors required for the caffeine response in Drosophila. Proc. Natl. Acad. Sci. 106, 4495-4500.

Levy, F., Bulet, P., and Ehret-Sabatier, L. (2004). Proteomic Analysis of the Systemic Immune Response of Drosophila. Mol. Cell. Proteomics 3, 156-166.

Li, M.A., Turner, D.J., Ning, Z., Yusa, K., Liang, Q., Eckert, S., Rad, L., Fitzgerald, T.W., Craig, N.L., and Bradley, A. (2011). Mobilization of giant piggyBac transposons in the mouse genome. Nucleic Acids Res. 39 , e148-e148.

Librado, P., and Rozas, J. (2013). Uncovering the functional constraints underlying the genomic organisation of the Odorant-Binding Protein genes. Genome Biol. Evol.

Linz, J., Baschwitz, A., Strutz, A., Dweck, H.K.M., Sachse, S., Hansson, B.S., and Stensmyr, M.C. (2013). Host plant-driven sensory specialization in Drosophila erecta. Proc. R. Soc. Lond. B Biol. Sci. 280, 20130626.

Liu, M., Yu, H., and Li, G. (2008). Oviposition deterrents from eggs of the cotton bollworm, Helicoverpa armigera (Lepidoptera: Noctuidae): chemical identification and analysis by electroantennogram. J. Insect Physiol. 54, 656-662.

Lorenzen, M.D., Berghammer, Brown, S.J., Denell, R.E., Klingler, M., and Beeman, R.W. (2003). piggyBac-mediated germline transformation in the beetle Tribolium castaneum. Insect Mol. Biol. 12, 433440.

Lundin, C., Käll, L., Kreher, S.A., Kapp, K., Sonnhammer, E.L., Carlson, J.R., Heijne, G. von, and Nilsson, I. (2007). Membrane topology of the Drosophila OR83b odorant receptor. FEBS Lett. 581, 5601-5604.

Maleszka, J., Forêt, S., Saint, R., and Maleszka, R. (2007). RNAi-induced phenotypes suggest a novel role for a chemosensory protein CSP5 in the development of embryonic integument in the honeybee (Apis mellifera). Dev. Genes Evol. 217, 189-196.

Martin, F., Boto, T., Gomez-Diaz, C., and Alcorta, E. (2013). Elements of Olfactory Reception in Adult Drosophila melanogaster. Anat. Rec. 296, 1477-1488.

Martin, J.R., Ernst, R., and Heisenberg, M. (1998). Mushroom bodies suppress locomotor activity in Drosophila melanogaster. Learn. Mem. Cold Spring Harb. N 5, 179-191.

Missbach, C., Dweck, H.K., Vogel, H., Vilcinskas, A., Stensmyr, M.C., Hansson, B.S., and Grosse-Wilde, E. (2014). Evolution of insect olfactory receptors. eLife 3. 
Miyazaki, T., and Ito, K. (2010). Neural architecture of the primary gustatory center of Drosophila melanogaster visualized with GAL4 and LexA enhancer-trap systems. J. Comp. Neurol. 518, 4147-4181.

Montell, C. (2009). A Taste of the Drosophila Gustatory Receptors. Curr. Opin. Neurobiol. 19, 345-353.

Mukunda, L., Lavista-Llanos, S., Hansson, B.S., and Wicher, D. (2014). Dimerisation of the Drosophila odorant coreceptor Orco. Front. Cell. Neurosci. 8.

Münch, D., and Galizia, C.G. (2016). DoOR 2.0 - Comprehensive Mapping of Drosophila melanogaster Odorant Responses. Sci. Rep. 6, 21841.

Nakai, J., Ohkura, M., and Imoto, K. (2001). A high signal-to-noise Ca2+ probe composed of a single green fluorescent protein. Nat. Biotechnol. 19, 137-141.

Ni, L., Bronk, P., Chang, E.C., Lowell, A.M., Flam, J.O., Panzano, V.C., Theobald, D.L., Griffith, L.C., and Garrity, P.A. (2013). A gustatory receptor paralogue controls rapid warmth avoidance in Drosophila. Nature 500, 580-584.

Nomura, A., Kawasaki, K., Kubo, T., and Natori, S. (1992). Purification and localization of p10, a novel protein that increases in nymphal regenerating legs of Periplaneta americana (American cockroach). Int. J. Dev. Biol. 36, 391-398.

Paczkowski, S., Paczkowska, M., Dippel, S., Flematti, G., and Schütz, S. (2014). Volatile Combustion Products of Wood Attract Acanthocnemus nigricans (Coleoptera: Acanthocnemidae). J. Insect Behav. 27, 228-238.

Pech, U., Dipt, S., Barth, J., Singh, P., Jauch, M., Thum, A.S., Fiala, A., and Riemensperger, T. (2013). Mushroom body miscellanea: transgenic Drosophila strains expressing anatomical and physiological sensor proteins in Kenyon cells. Front. Neural Circuits 7, 147.

Pedigo, L.P., and Rice, M.E. (2014). Entomology and Pest Management: Sixth Edition (Waveland Press).

Pelletier, J., and Leal, W.S. (2009). Genome Analysis and Expression Patterns of Odorant-Binding Proteins from the Southern House Mosquito Culex pipiens quinquefasciatus. PLoS ONE 4, e6237.

Pelletier, J., Guidolin, A., Syed, Z., Cornel, A.J., and Leal, W.S. (2010). Knockdown of a Mosquito Odorant-binding Protein Involved in the Sensitive Detection of Oviposition Attractants. J. Chem. Ecol. 36, 245-248.

Pelosi, P., lovinella, I., Felicioli, A., and Dani, F.R. (2014). Soluble proteins of chemical communication: an overview across arthropods. Integr. Physiol. 5, 320.

Pelz, D., Roeske, T., Syed, Z., Bruyne, M. de, and Galizia, C.G. (2006). The molecular receptive range of an olfactory receptor in vivo (Drosophila melanogaster Or22a). J. Neurobiol. 66, 1544-1563.

Pfeiffer, B.D., Ngo, T.-T.B., Hibbard, K.L., Murphy, C., Jenett, A., Truman, J.W., and Rubin, G.M. (2010). Refinement of Tools for Targeted Gene Expression in Drosophila. Genetics 186, 735-755.

Pitts, R.J., Rinker, D.C., Jones, P.L., Rokas, A., and Zwiebel, L.J. (2011). Transcriptome profiling of chemosensory appendages in the malaria vector Anopheles gambiae reveals tissue- and sex-specific signatures of odor coding. BMC Genomics 12, 271.

Qiao, H., He, X., Schymura, D., Ban, L., Field, L., Dani, F.R., Michelucci, E., Caputo, B., Torre, A. della, latrou, K., et al. (2011). Cooperative interactions between odorant-binding proteins of Anopheles gambiae. Cell. Mol. Life Sci. 68, 1799-1813. 
Richards, S., Gibbs, R.A., Weinstock, G.M., Brown, S.J., Denell, R., Beeman, R.W., Gibbs, R., Beeman, R.W., Brown, S.J., Bucher, G., et al. (2008). The genome of the model beetle and pest Tribolium castaneum. Nature 452, 949-955.

Ridley, A.W., Hereward, J.P., Daglish, G.J., Raghu, S., Collins, P.J., and Walter, G.H. (2011). The spatiotemporal dynamics of Tribolium castaneum (Herbst): adult flight and gene flow. Mol. Ecol. 20, 1635-1646.

Riemensperger, T., Kittel, R.J., and Fiala, A. (2016). Optogenetics in Drosophila Neuroscience. Methods Mol. Biol. Clifton NJ 1408, 167-175.

Rinker, D.C., Pitts, R.J., Zhou, X., Suh, E., Rokas, A., and Zwiebel, L.J. (2013). Blood meal-induced changes to antennal transcriptome profiles reveal shifts in odor sensitivities in Anopheles gambiae. Proc. Natl. Acad. Sci. U. S. A. 110, 8260-8265.

Robertson, H.M., and Kent, L.B. (2009). Evolution of the gene lineage encoding the carbon dioxide receptor in insects. J. Insect Sci. Online 9, 19.

Roth, L.M., and Willis, E.R. (1951). Hygroreceptors in Coleoptera. J. Exp. Zool. 117, 451-487.

Rubin, G.M., and Spradling, A.C. (1982). Genetic transformation of Drosophila with transposable element vectors. Science $218,348-353$.

Rytz, R., Croset, V., and Benton, R. (2013). Ionotropic receptors (IRs): chemosensory ionotropic glutamate receptors in Drosophila and beyond. Insect Biochem. Mol. Biol. 43, 888-897.

Sachse, S. (2014). Coding of odor valence and intensity in the Drosophila olfactory circuit. Flavour 3, 1-1.

Sallam, M.N., and Mejida, D. (2008). INSECT DAMAGE: Damage on Post-harvest. Food Agric. Organ. U. $\mathrm{N}$.

Sánchez-Gracia, A., Vieira, F.G., Almeida, F.C., and Rozas, J. (2001). Comparative Genomics of the Major Chemosensory Gene Families in Arthropods. In eLS, (John Wiley \& Sons, Ltd), p.

Sandler, B.H., Nikonova, L., Leal, W.S., and Clardy, J. (2000). Sexual attraction in the silkworm moth: structure of the pheromone-binding-protein-bombykol complex. Chem. Biol. 7, 143-151.

Sarkar, A., Sim, C., Hong, Y.S., Hogan, J.R., Fraser, M.J., Robertson, H.M., and Collins, F.H. (2003). Molecular evolutionary analysis of the widespread piggyBac transposon family and related "domesticated" sequences. Mol. Genet. Genomics 270, 173-180.

Sato, K., Pellegrino, M., Nakagawa, T., Nakagawa, T., Vosshall, L.B., and Touhara, K. (2008). Insect olfactory receptors are heteromeric ligand-gated ion channels. Nature 452, 1002-1006.

Sato, K., Tanaka, K., and Touhara, K. (2011). Sugar-regulated cation channel formed by an insect gustatory receptor. Proc. Natl. Acad. Sci. 108, 11680-11685.

Savard, J., Marques-Souza, H., Aranda, M., and Tautz, D. (2006). A Segmentation Gene in Tribolium Produces a Polycistronic mRNA that Codes for Multiple Conserved Peptides. Cell 126, 559-569.

Scaloni, A., Monti, M., Angeli, S., and Pelosi, P. (1999). Structural analysis and disulfide-bridge pairing of two odorant-binding proteins from Bombyx mori. Biochem. Biophys. Res. Commun. 266, 386-391.

Schachtner, J., Schmidt, M., and Homberg, U. (2005). Organization and evolutionary trends of primary olfactory brain centers in Tetraconata (Crustacea+Hexapoda). Arthropod Struct. Dev. 34, 257-299. 
Schetelig, M.F., and Wimmer, E.A. (2011). Insect Transgenesis and the Sterile Insect Technique. In Insect Biotechnology, A. Vilcinskas, ed. (Springer Netherlands), pp. 169-194.

Schinko, J.B., Weber, M., Viktorinova, I., Kiupakis, A., Averof, M., Klingler, M., Wimmer, E.A., and Bucher, G. (2010). Functionality of the GAL4/UAS system in Tribolium requires the use of endogenous core promoters. BMC Dev. Biol. 10, 53.

Schinko, J.B., Hillebrand, K., and Bucher, G. (2012). Heat shock-mediated misexpression of genes in the beetle Tribolium castaneum. Dev. Genes Evol. 222, 287-298.

Schmitt-Engel, C., Schultheis, D., Schwirz, J., Ströhlein, N., Troelenberg, N., Majumdar, U., Dao, V.A., Grossmann, D., Richter, T., Tech, M., et al. (2015). The iBeetle large-scale RNAi screen reveals gene functions for insect development and physiology. Nat. Commun. 6, 7822.

Schröter, U., and Menzel, R. (2003). A new ascending sensory tract to the calyces of the honeybee mushroom body, the subesophageal-calycal tract: Sensory Inputs of The Mushroom Body. J. Comp. Neurol. 465, 168-178.

Schultze, A., Pregitzer, P., Walter, M.F., Woods, D.F., Marinotti, O., Breer, H., and Krieger, J. (2013). The Co-Expression Pattern of Odorant Binding Proteins and Olfactory Receptors Identify Distinct Trichoid Sensilla on the Antenna of the Malaria Mosquito Anopheles gambiae. PLoS ONE 8, e69412.

Scott, K., Brady, R., Jr, Cravchik, A., Morozov, P., Rzhetsky, A., Zuker, C., and Axel, R. (2001). A chemosensory gene family encoding candidate gustatory and olfactory receptors in Drosophila. Cell 104, 661-673.

Serrano, J.M., Castro, L., Toro, M.A., and López-Fanjul, C. (1991). The genetic properties of homosexual copulation behavior in Tribolium castaneum: diallel analysis. Behav. Genet. 21, 547-558.

Shanbhag, S.R., Müller, B., and Steinbrecht, R.A. (1999). Atlas of olfactory organs of Drosophila melanogaster: 1. Types, external organization, innervation and distribution of olfactory sensilla. Int. J. Insect Morphol. Embryol. 28, 377-397.

Shearin, H.K., Macdonald, I.S., Spector, L.P., and Stowers, R.S. (2014). Hexameric GFP and mCherry Reporters for the Drosophila GAL4, Q, and LexA Transcription Systems. Genetics 196, 951-960.

Shim, J., Lee, Y., Jeong, Y.T., Kim, Y., Lee, M.G., Montell, C., and Moon, S.J. (2015). The full repertoire of Drosophila gustatory receptors for detecting an aversive compound. Nat. Commun. 6, 8867.

Shinmyo, Y., Mito, T., Matsushita, T., Sarashina, I., Miyawaki, K., Ohuchi, H., and Noji, S. (2004). piggyBac-mediated somatic transformation of the two-spotted cricket, Gryllus bimaculatus. Dev. Growth Differ. 46, 343-349.

Shurin, J.B., Gruner, D.S., and Hillebrand, H. (2006). All wet or dried up? Real differences between aquatic and terrestrial food webs. Proc. R. Soc. Lond. B Biol. Sci. 273, 1-9.

Silbering, A.F., Rytz, R., Grosjean, Y., Abuin, L., Ramdya, P., Jefferis, G.S.X.E., and Benton, R. (2011). Complementary function and integrated wiring of the evolutionarily distinct Drosophila olfactory subsystems. J. Neurosci. Off. J. Soc. Neurosci. 31, 13357-13375.

Smith, C. (2008). Biology of Sensory Systems (John Wiley \& Sons).

Sokoloff, A. (1972). The biology of Tribolium. Vol. 1. Clarendon (Oxford).

Steinbrecht, R.A. (1996). Structure and function in insect olfactory sensilla. Ciba Found. Symp. 200, 15874-7. 
Steinbrecht, R.A. (1998). Odorant-binding proteins: expression and function. Ann. N. Y. Acad. Sci. 855, 323-332.

Steinbrecht, R.A., Laue, M., and Ziegelberger, G. (1995). Immunolocalization of pheromone-binding protein and general odorant-binding protein in olfactory sensilla of the silk moths Antheraea and Bombyx. Cell Tissue Res. 282, 203-217.

Stensmyr, M.C., Dweck, H.K.M., Farhan, A., Ibba, I., Strutz, A., Mukunda, L., Linz, J., Grabe, V., Steck, K., Lavista-Llanos, S., et al. (2012). A Conserved Dedicated Olfactory Circuit for Detecting Harmful Microbes in Drosophila. Cell 151, 1345-1357.

Stocker, R.F. (2001). Drosophila as a focus in olfactory research: mapping of olfactory sensilla by fine structure, odor specificity, odorant receptor expression, and central connectivity. Microsc. Res. Tech. 55, 284-296.

Strutz, A., Soelter, J., Baschwitz, A., Farhan, A., Grabe, V., Rybak, J., Knaden, M., Schmuker, M., Hansson, B.S., and Sachse, S. (2014). Decoding odor quality and intensity in the Drosophila brain. eLife 3, e04147.

Sun, Y.-L., Huang, L.-Q., Pelosi, P., and Wang, C.-Z. (2012). Expression in Antennae and Reproductive Organs Suggests a Dual Role of an Odorant-Binding Protein in Two Sibling Helicoverpa Species. PLoS ONE 7, e30040.

Suzuki, T. (1980). 4, 8-Dimethyldecanal: The aggregation pheromone of the flour beetles, Tribolium castaneum and T. confusum (Coleoptera: Tenebrionidae). Agric. Biol. Chem. 44, 2519-2520.

Suzuki, T., Nakakita, H., and Kuwahara, Y. (1988). Cross-attraction among three Tribolium species and their hybrids(Coleoptera: Tenebrionidae). Appl. Entomol. Zool. 23, 477-480.

Swarup, S., Williams, T.I., and Anholt, R.R.H. (2011). Functional dissection of Odorant binding protein genes in Drosophila melanogaster. Genes Brain Behav. 10, 648-657.

Szyszka, P., and Galizia, C.G. (2015). Olfaction in Insects. In Handbook of Olfaction and Gustation, R.L. Doty, ed. (John Wiley \& Sons, Inc), pp. 531-546.

Tanaka, N.K., Tanimoto, H., and Ito, K. (2008). Neuronal assemblies of the Drosophila mushroom body. J. Comp. Neurol. 508, 711-755.

Tomoyasu, Y., and Denell, R.E. (2004). Larval RNAi in Tribolium (Coleoptera) for analyzing adult development. Dev. Genes Evol. 214, 575-578.

Trauner, J., Schinko, J., Lorenzen, M.D., Shippy, T.D., Wimmer, E.A., Beeman, R.W., Klingler, M., Bucher, G., and Brown, S.J. (2009). Large-scale insertional mutagenesis of a coleopteran stored grain pest, the red flour beetle Tribolium castaneum, identifies embryonic lethal mutations and enhancer traps. BMC Biol. 7, 73.

Verheggen, F., Ryne, C., Olsson, P.-O.C., Arnaud, L., Lognay, G., Högberg, H.-E., Persson, D., Haubruge, E., and Löfstedt, C. (2007). Electrophysiological and Behavioral Activity of Secondary Metabolites in the Confused Flour Beetle, Tribolium confusum. J. Chem. Ecol. 33, 525-539.

Vieira, F.G., and Rozas, J. (2011). Comparative Genomics of the Odorant-Binding and Chemosensory Protein Gene Families across the Arthropoda: Origin and Evolutionary History of the Chemosensory System. Genome Biol. Evol. 3, 476-490.

Vogt, R.G., and Riddiford, L.M. (1981). Pheromone binding and inactivation by moth antennae. Nature 293, 161-163. 
Vogt, K., Aso, Y., Hige, T., Knapek, S., Ichinose, T., Friedrich, A.B., Turner, G.C., Rubin, G.M., and Tanimoto, H. (2016). Direct neural pathways convey distinct visual information to Drosophila mushroom bodies. eLife 5, e14009.

Vosshall, L.B. (2000). Olfaction in Drosophila. Curr. Opin. Neurobiol. 10, 498-503.

Vosshall, L.B., and Hansson, B.S. (2011). A Unified Nomenclature System for the Insect Olfactory Coreceptor. Chem. Senses bjr022.

Vosshall, L.B., and Stocker, R.F. (2007). Molecular architecture of smell and taste in Drosophila. Annu. Rev. Neurosci. 30, 505-533.

Wang, B., Guan, L., Zhong, T., Li, K., Yin, J., and Cao, Y. (2013). Potential Cooperations between Odorant-Binding Proteins of the Scarab Beetle Holotrichia oblita Faldermann (Coleoptera: Scarabaeidae). PLoS ONE 8, e84795.

Wang, L., Wang, S., Li, Y., Paradesi, M.S.R., and Brown, S.J. (2007). BeetleBase: the model organism database for Tribolium castaneum. Nucleic Acids Res. 35, D476-D479.

Watanabe, T., Ochiai, H., Sakuma, T., Horch, H.W., Hamaguchi, N., Nakamura, T., Bando, T., Ohuchi, H., Yamamoto, T., Noji, S., et al. (2012). Non-transgenic genome modifications in a hemimetabolous insect using zinc-finger and TAL effector nucleases. Nat. Commun. 3, 1017.

Weiss, L.A., Dahanukar, A., Kwon, J.Y., Banerjee, D., and Carlson, J.R. (2011). The Molecular and Cellular Basis of Bitter Taste in Drosophila. Neuron 69, 258-272.

Wetzel, C.H., Behrendt, H.J., Gisselmann, G., Störtkuhl, K.F., Hovemann, B., and Hatt, H. (2001). Functional expression and characterization of a Drosophila odorant receptor in a heterologous cell system. Proc. Natl. Acad. Sci. U. S. A. 98, 9377-9380.

Whiteman, N.K., and Pierce, N.E. (2008). Delicious poison: genetics of Drosophila host plant preference. Trends Ecol. Evol. 23, 473-478.

Wicher, D., Schäfer, R., Bauernfeind, R., Stensmyr, M.C., Heller, R., Heinemann, S.H., and Hansson, B.S. (2008). Drosophila odorant receptors are both ligand-gated and cyclic-nucleotide-activated cation channels. Nature 452, 1007-1011.

Wright, J.A., Smith, R.C., Xie, K., Craig, N.L., and Atkinson, P.W. (2013). IPB7 transposase behavior in Drosophila melanogaster and Aedes aegypti. Insect Biochem. Mol. Biol. 43, 899-906.

Yang, C.-H., Belawat, P., Hafen, E., Jan, L.Y., and Jan, Y.-N. (2008). Drosophila egg-laying site selection as a system to study simple decision-making processes. Science $319,1679-1683$.

Yeh, E., Gustafson, K., and Boulianne, G.L. (1995). Green fluorescent protein as a vital marker and reporter of gene expression in Drosophila. Proc. Natl. Acad. Sci. U. S. A. 92, 7036-7040.

Yezerski, A., Ciccone, C., Rozitski, J., and Volingavage, B. (2007). The Effects of a Naturally Produced Benzoquinone on Microbes Common to Flour. J. Chem. Ecol. 33, 1217-1225.

Younus, F., Chertemps, T., Pearce, S.L., Pandey, G., Bozzolan, F., Coppin, C.W., Russell, R.J., Maïbèche-Coisne, M., and Oakeshott, J.G. (2014). Identification of candidate odorant degrading gene/enzyme systems in the antennal transcriptome of Drosophila melanogaster. Insect Biochem. Mol. Biol. 53, 30-43.

Yusa, K., Zhou, L., Li, M.A., Bradley, A., and Craig, N.L. (2011). A hyperactive piggyBac transposase for mammalian applications. Proc. Natl. Acad. Sci. 108, 1531-1536. 
Zeng, V., Ewen-Campen, B., Horch, H.W., Roth, S., Mito, T., and Extavour, C.G. (2013). Developmental gene discovery in a hemimetabolous insect: de novo assembly and annotation of a transcriptome for the cricket Gryllus bimaculatus. PloS One 8, e61479.

Zhang, W., Ricketts, T.H., Kremen, C., Carney, K., and Swinton, S.M. (2007). Ecosystem services and dis-services to agriculture. Ecol. Econ. 64, 253-260.

Zhang, Y.-N., Jin, J.-Y., Jin, R., Xia, Y.-H., Zhou, J.-J., Deng, J.-Y., and Dong, S.-L. (2013a). Differential Expression Patterns in Chemosensory and Non-Chemosensory Tissues of Putative Chemosensory Genes Identified by Transcriptome Analysis of Insect Pest the Purple Stem Borer Sesamia inferens (Walker). PLoS ONE 8.

Zhang, Y.V., Ni, J., and Montell, C. (2013b). The Molecular Basis for Attractive Salt-Taste Coding in Drosophila. Science 340, 1334-1338.

Zhao, Y., Araki, S., Wu, J., Teramoto, T., Chang, Y.-F., Nakano, M., Abdelfattah, A.S., Fujiwara, M., Ishihara, T., Nagai, T., et al. (2011). An Expanded Palette of Genetically Encoded Ca2+ Indicators. Science 333, 1888-1891.

Zheng, W., Peng, W., Zhu, C., Zhang, Q., Saccone, G., and Zhang, H. (2013). Identification and Expression Profile Analysis of Odorant Binding Proteins in the Oriental Fruit Fly Bactrocera dorsalis. Int. J. Mol. Sci. 14, 14936-14949.

Zhou, J.-J., Kan, Y., Antoniw, J., Pickett, J.A., and Field, L.M. (2006). Genome and EST Analyses and Expression of a Gene Family with Putative Functions in Insect Chemoreception. Chem. Senses 31, 453465. 


\section{$6 \quad$ Appendix}

\subsection{Abbrevations}

ABP II antennal binding protein II

$\mathrm{AL}$ antennal lobe

ALDH aldehyde dehydrogenase

ALOX aldehyde oxidase

ALT antennal lobe tract

CA calyx of the MB

CES carboxylesterase

CSN chemosensory neuron

CSP chemosensory protein

cSTri chemosensilla trichoidea

CYP cytochrome P450

dsRed Discosoma sp. red fluorescent protein

EH epoxide hydrolase

GABA gamma-Aminobutyric acid

GFP green fluorescent protein

GL antennal lobe glomeruli

GNG gnathal ganglia

GOC gnathal olfactory center

GR gustatory receptor

GSN gustatory sensory neuron

GST glutathione S-transferase

IHC Immunohistochemistry

IN local interneuron

IR Ionotropic glutamate-like receptor

-ir immunoreactive

KC kenyon cells

LG lobus glomerulatus

$\mathrm{LH} \quad$ lateral horn (lateral protocerebrum)

MB mushroom body

mSTri mechanosensilla trichoidea

OBP odorant binding protein

ODE odorant degrading enzyme

OR odorant receptor

Orco odorant receptor coreceptor

ORF open reading frame

OSN olfactory sensory neurons

PGC primary gustatory center

$\mathrm{PN} \quad$ projection neuron

RPKM reads per kilobase per million

SBas sensilla basiconica

Scam sensilla campaniformes

SCha sensilla chaetica

SCoe sensilla coeloconica

SEM scanning electron microscope

SNMP sensory neuron membrane protein

SpaB spaculate bristle

TKRP tachykinin related peptide

TRE tetracycline resonding element

tTA tetracycline transactivator

UAS upstream activation sequence 


\subsection{Vector maps and sequences}
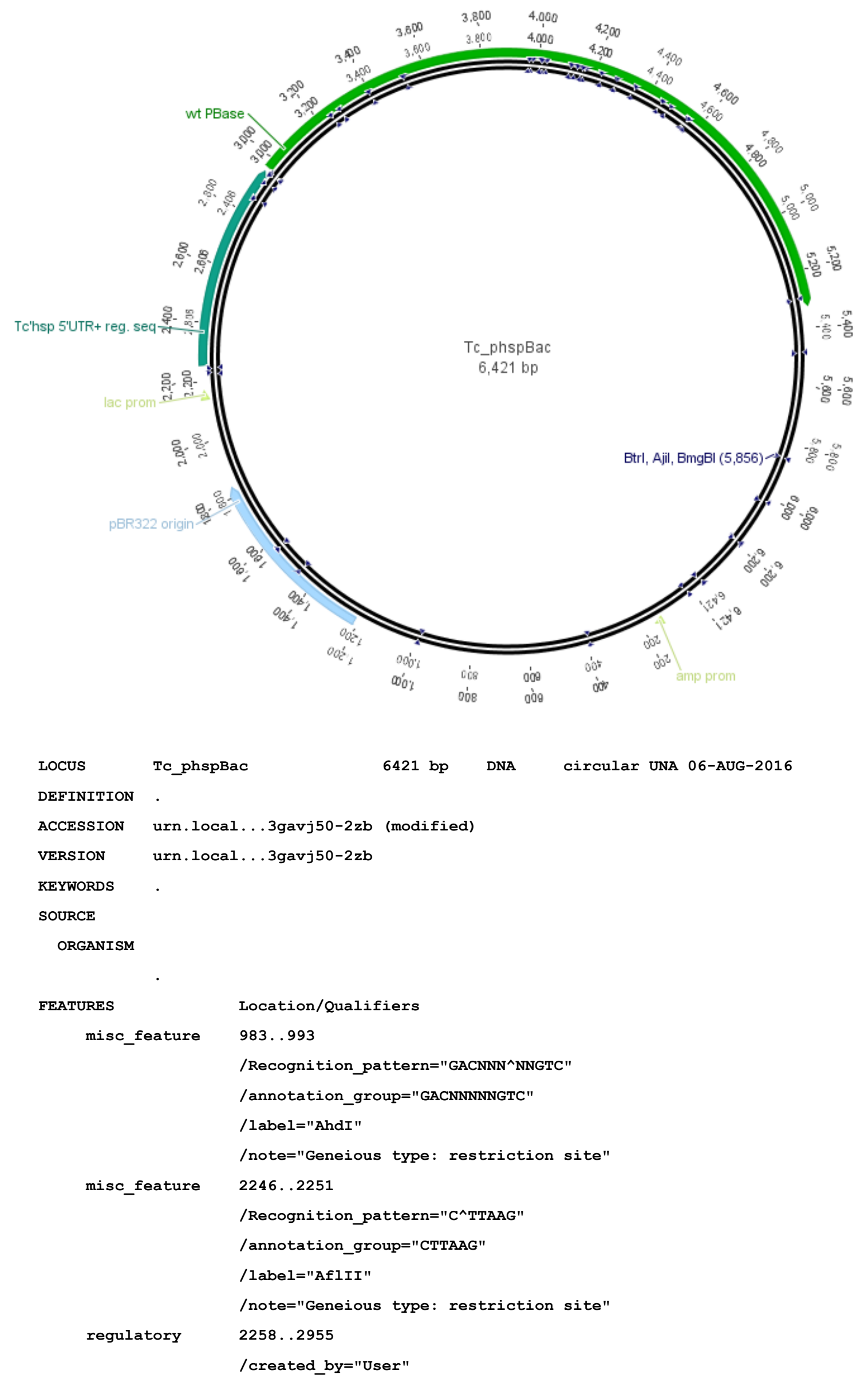
/modified_by="User"

/label="Tc_hsp68"

misc_feature

$2919 . .2924$

/Recognition_pattern="A^CTAGT"

/annotation_group="ACTAGT"

/label="SpeI"

/note="Geneious type: restriction site"

misc_feature $\quad 2951.2956$

/Recognition_pattern="T^CTAGA"

/annotation_group="TCTAGA"

/label="XbaI"

/note="Geneious type: restriction site"

misc_feature

2957 . 2962

/Recognition_pattern="G^GTACC"

/annotation_group="GGTACC"

/1abel="Acc65I"

/note="Geneious type: restriction site"

misc_feature 2957.2962

/Recognition_pattern="GGTAC^C"

/annotation_group="GGTACC"

/label="KpnI"

/note="Geneious type: restriction site"

ORF

2972 . 5324

/modified_by="User"

/label="wt PBase"

misc_feature

$3526 \ldots 3531$

/Recognition_pattern="A^GATCT"

/annotation_group="AGATCT"

/label="BglII"

/note="Geneious type: restriction site"

misc_feature $4023 \ldots 4032$

/Recognition_pattern="GATNN^NNATC"

/annotation_group="GATNNNNATC"

/label="BsaBI"

/note="Geneious type: restriction site"

misc_feature

4148 . 4156

/Recognition_pattern="CACNNN^GTG"

/annotation_group="CACNNNGTG"

/label="DraIII"

/note="Geneious type: restriction site"

misc_feature $\quad 4218 \ldots 4223$

/Recognition_pattern="C^AATTG"

/annotation_group="CAATTG"

/label="MfeI"

/note="Geneious type: restriction site"

misc feature

4272 . . 4277

/Recognition_pattern="GTT^AAC"

/annotation_group="GTTAAC" 
/label="HpaI"

/note="Geneious type: restriction site"

misc_feature $\quad 4340 \ldots 4351$

/Recognition_pattern="CCANNNNN^NTGG"

/annotation group="CCANNNNNNTGG"

/label="BstXI"

/note="Geneious type: restriction site"

misc_feature $\quad 4549 \ldots 4554$

/Recognition_pattern="ACCTGC (4/8) "

/annotation_group="АCCTGC"

/label="BfuAI"

/note="Geneious type: restriction site"

misc_feature $\quad 4551.4556$

/Recognition_pattern="CTGCA^G"

/annotation_group="CTGCAG"

/label="PstI"

/note="Geneious type: restriction site"

misc_feature $\quad 5294.5299$

/Recognition_pattern="C^GTACG"

/annotation_group="CGTACG"

/label="BsiWI"

/note="Geneious type: restriction site"

misc_feature $\quad 6018.6023$

/Recognition_pattern="A^AGCTT"

/annotation_group="AAGCTT"

/label="HindIII"

/note="Geneious type: restriction site"

ORIGIN

1 gacgaaaggg cctcgtgata cgcctatttt tataggttaa tgtcatgata ataatggttt

61 cttagacgtc aggtggcact tttcggggaa atgtgcgcgg aacccctatt tgtttatttt

121 tctaaataca ttcaaatatg tatccgctca tgagacaata accctgataa atgcttcaat

181 aatattgaaa aaggaagagt atgagtattc aacatttccg tgtcgccctt attccctttt

241 ttgcggcatt ttgccttcct gtttttgctc acccagaaac gctggtgaaa gtaaaagatg

301 ctgaagatca gttgggtgca cgagtgggtt acatcgaact ggatctcaac agcggtaaga

361 tccttgagag ttttcgcccc gaagaacgtt ttccaatgat gagcactttt aaagttctgc

421 tatgtggcgc ggtattatcc cgtattgacg ccgggcaaga gcaactcggt cgccgcatac

481 actattctca gaatgacttg gttgagtact caccagtcac agaaagcat cttacggatg

541 gcatgacagt aagagaatta tgcagtgctg ccataaccat gagtgataac actgcggcca

601 acttacttct gacaacgatc ggaggaccga aggagctaac cgcttttttg cacaacatgg

661 gggatcatgt aactcgcctt gatcgttggg aaccggagct gaatgaagcc ataccaaacg

721 acgagcgtga caccacgatg cctgtagcaa tggcaacaac gttgcgcaaa ctattaactg

781 gcgaactact tactctagct tcccggcaac aattaataga ctggatggag gcggataaag

841 ttgcaggacc acttctgcgc tcggccettc cggctggctg gtttattgct gataaatctg

901 gagccggtga gcgtgggtct cgcggtatca ttgcagcact ggggccagat ggtaagccet

961 cccgtatcgt agttatctac acgacgggga gtcaggcaac tatggatgaa cgaaatagac

1021 agatcgctga gataggtgcc tcactgatta agcattggta actgtcagac caagtttact

1081 catatatact ttagattgat ttaaacttc atttttaatt taaaggatc taggtgaaga

1141 tcctttttga taatctcatg accaaatcc cttaacgtga gttttcgttc cactgagcgt 
1201 cagaccccgt agaaaagatc aaaggatctt cttgagatcc tttttttctg cgcgtaatct

1261 gctgcttgca aacaaaaaa ccaccgctac cagcggtggt ttgtttgccg gatcaagagc 1321 taccaactct ttttccgaag gtaactggct tcagcagagc gcagatacca aatactgtcc 1381 ttctagtgta gccgtagtta ggccaccact tcaagaactc tgtagcaccg cctacatacc 1441 tcgctctgct aatcctgtta ccagtggctg ctgccagtgg cgataagtcg tgtcttaccg 1501 ggttggactc aagacgatag ttaccggata aggcgcagcg gtcgggctga acggggggtt 1561 cgtgcacaca gcccagcttg gagcgaacga cctacaccga actgagatac ctacagcgtg 1621 agcattgaga aagcgccacg cttcccgaag ggagaaaggc ggacaggtat ccggtaagcg 1681 gcagggtcgg aacaggagag cgcacgaggg agcttccagg gggaaacgcc tggtatcttt 1741 atagtcctgt cgggtttcgc cacctctgac ttgagcgtcg atttttgtga tgctcgtcag 1801 gggggcggag cctatggaaa aacgccagca acgcggcctt tttacggttc ctggcetttt 1861 gctggccttt tgctcacatg ttctttcctg cgttatcccc tgattctgtg gataaccgta 1921 ttaccgcett tgagtgagct gataccgctc gccgcagccg aacgaccgag cgcagcgagt 1981 cagtgagcga ggaagcggaa gagcgcccaa tacgcaaacc gcctctcccc gcgcgttggc 2041 cgattcatta atgcagctgg cacgacaggt ttcccgactg gaaagcgggc agtgagcgca 2101 acgcaattaa tgtgagttag ctcactcatt aggcacccca ggctttacac tttatgcttc 2161 cggctcgtat gttgtgtgga attgtgagcg gataacaatt tcacacagga aacagctatg 2221 accatgatta cggtgggccg tgcaccttaa gctgatccca accttcaata aaaattgtt 2281 ctttatttat tattaatcac tattatttag gcatcttctg caaagtaat tgattataaa 2341 cgataaaagt aaaaaaagg attaatctaa aacattttac ttaaagacct attaataagt 2401 ttaaaagaa aaaaatagg aaautgttt tattaaaut gctatgacaa tccaactttg 2461 gcatatgtat atgaaatcaa aacaattcac tactcattca taatattgcc gaactttacc 2521 aaattaaaa taaacatgaa atagtcaaca actcattcgt tatgtaacgt ttttttttta 2581 aatgcattaa tatataatga ttgtttttat tttggaagtt gaaataatt agggcacaac 2641 cagtttccac tattctcctt tatctttacg gaaatagcat gaataatacc gaaaggccg 2701 ttttaaaat aaaattcga gaaaattcaa acgcaaatca atggaaactt cgagatcatt 2761 cggtgaagtt cgagacgaat tccccgtttc atatataagc gcggtctcgc ggcgcgttgt 2821 cagtgaattt gaatcaagcg aagcgaaagc aacaaagcta agcaagcgac tcaaagcaaa 2881 ttttcaaagc gactttaaca aagttgagtg aattatttac tagtgaattc aaagtaaatc 2941 gaaagtgca tctagaggta ccggttgttg gccogtgagg cgtgcttgtc aatgcggtaa 3001 gtgtcactga ttttgaacta taacgaccgc gtgagtcaaa atgacgcatg attatctttt 3061 acgtgacttt taagatttaa ctcatacgat aattatattg ttattcatg ttctacttac 3121 gtgataactt attatatata tattttcttg ttatagatat cgtgactaat atataataaa 3181 atgggtagtt ctttagacga tgagcatatc ctctctgctc ttctgcaaag cgatgacgag 3241 cttgttggtg aggattctga cagtgaaata tcagatcacg taagtgaaga tgacgtccag 3301 agcgatacag aagaagcgtt tatagatgag gtacatgaag tgcagccaac gtcaagcggt 3361 agtgaaatat tagacgaaca aaatgttatt gaacaaccag gttcttcatt ggcttctaac 3421 agaatcttga ccttgccaca gaggactatt agaggtaaga ataaacattg ttggtcaact 3481 tcaaagtcca cgaggcgtag ccgagtctct gcactgaaca ttgtcagatc tcaaagaggt 3541 ccgacgcgta tgtgccgcaa tatatatgac ccacttttat gcttcaaact attttttact 3601 gatgagataa tttcggaaat tgtaaatgg acaaatgctg agatatcatt gaaacgtcgg 3661 gaatctatga caggtgctac atttcgtgac acgaatgaag atgaaatcta tgctttcttt 3721 ggtattctgg taatgacagc agtgagaaaa gataaycaca tgtccacaga tgacctcttt 3781 gatcgatctt tgtcaatggt gtacgtctct gtaatgagtc gtgatcgttt tgattttttg 3841 atacgatgtc ttagaatgga tgacaaagt atacggccca cacttcgaga aaacgatgta 3901 tttactcctg ttagaaaaat atgggatctc tttatccatc agtgcataca aaattacact 3961 ccaggggctc atttgaccat agatgaacag ttacttggtt ttagaggacg gtgtccgttt 4021 aggatgtata tcccaaacaa gccaagtaag tatggaataa aaatcctcat gatgtgtgac 
4081 agtggtacga agtatatgat aaatggaatg ccttatttgg gaagaggaac acagaccaac

4141 ggagtaccac tcggtgaata ctacgtgaag gagttatcaa agcctgtgca cggtagttgt 4201 cgtaatatta cgtgtgacaa ttggttcacc tcaatccctt tggcaaaaa cttactacaa 4261 gaaccgtata agttaaccat tgtgggaacc gtgcgatcaa acaaacgcga gataccggaa 4321 gtactgaaaa acagtcgctc caggccagtg ggaacatcga tgttttgttt tgacggaccc 4381 cttactctcg tctcatataa accgaagcca gctaagatgg tatacttatt atcatcttgt 4441 gatgaggatg cttctatcaa cgaaagtacc ggtaaaccgc aaatggttat gtattataat 4501 caaactaaag gcggagtgga cacgctagac caaatgtgtt ctgtgatgac ctgcagtagg 4561 aagacgaata ggtggcctat ggcattattg tacggaatga taacattgc ctgcataaat 4621 tcttttatta tatacagcca taatgtcagt agcaagggag aaaggtcca aagtcgcaaa 4681 aaatttatga gaaccttta catgagcctg acgtcatcgt ttatgcgtaa gcgtttagaa 4741 gctcctactt tgaagagata tttgcgcgat aatatctcta atattttgcc aaatgaagtg 4801 cctggtacat cagatgacag tactgaagag ccagtaatga aaaacgtac ttactgtact 4861 tactgcccet ctaaaataag gcgaaaggca aatgcatcgt gcaaaaatg caaaaaagt 4921 atttgtcgag agcataatat tgatatgtgc caaagttgtt tctgactgac taataagtat 4981 aatttgtttc tattatgtat aagttaagct aattacttat tttataatac aacatgactg 5041 tttttaaagt acaaaataag tttatttttg taaagagag aatgtttaaa agttttgtta 5101 ctttatagaa gaaattttga gtttttgttt ttttttaata aataaataaa cataaataaa 5161 ttgtttgttg aatttattat tagtatgtaa gtgtaaatat aataaactt aatatctatt 5221 caaattaata aataacctc gatatacaga ccgataaac acatgcgtca attttacgca 5281 tgattatctt taacgtacgt cacaatatga ttatctttct agggttaaat aatagtttct 5341 aattttttta ttattcagcc tgctgtcgtg aataccgtat atctcaacgc tgtctgtgag 5401 attgtcgtat tctagccttt ttagtttttc gctcatcgac ttgatattgt ccgacacatt 5461 ttcgtcgatt tgcgttttga tcaaagactt gagcagagac acgttaatca actgttcaaa 5521 ttgatccata ttaacgatat caacccgatg cgtatatggt gcgtaaata tattttttaa 5581 ccctcttata ctttgcactc tgcgttaata cgcgttcgtg tacagacgta atcatgtttt 5641 cttttttgga taaactcct actgagtttg acctcatatt agaccctcac aagttgcaaa 5701 acgtggcatt ttttaccaat gaagaattta aagttatttt aaaaatttc atcacagatt 5761 taaagaagaa ccaaaatta aattatttca acagtttaat cgaccagtta atcaacgtgt 5821 acacagacgc gtcggcaaaa aacacgcagc ccgacgtgtt ggctaaatt attaaatcaa 5881 cttgtgttat agtcacggat ttgccgtcca acgtgttcct caaaagttg aagaccaaca 5941 agtttacgga cactattaat tatttgattt tgccccactt cattttgtgg gatcacaatt 6001 ttgttatatt ttaaacaaag cttggcactg gccgtcgttt tacaacgtcg tgactgggaa 6061 aaccetggcg ttacccaact taatcgcctt gcagcacatc cccctttcgc cagctggcgt 6121 aatagcgaag aggcccgcac cgatcgccct tcccaacagt tgcgcagcct gaatggcgaa 6181 tggcgcctga tgcggtattt tctccttacg catctgtgcg gtatttcaca ccgcatatgg 6241 tgcactctca gtacaatctg ctctgatgcc gcatagttaa gccagccco acacccgcca 6301 acacccgctg acgcgccetg acgggcttgt ctgctcccgg catccgctta cagacaagct 6361 gtgaccgtct ccgggagctg catgtgtcag aggttttcac cgtcatcacc gaaacgcgcg 6421 a 
122

Appendix
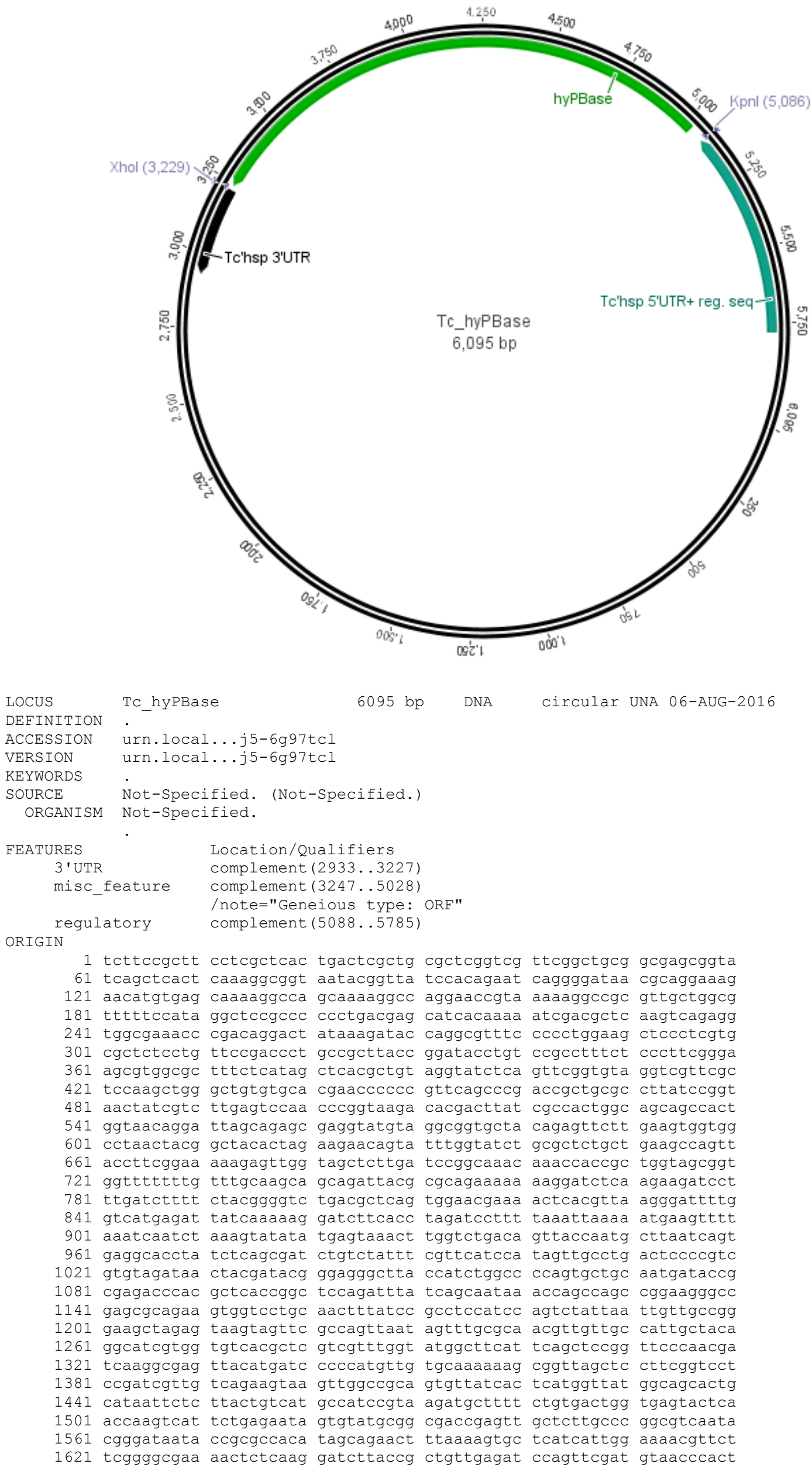

FEATURES

3 'UTR

misc_feature

regulatory

ORIGIN

1 tcttccgct cctcgctcac tgactcgctg cgctcggtcg ttcggctgcg gcgagcggta

61 tcagctcact caaaggcggt aatacggtta tccacagaat caggggataa cgcaggaaag

121 aacatgtgag caaaaggcca gcaaaaggcc aggaaccgta aaaaggccgc gttgctggcg

181 tttttccata ggctccgccc ccctgacgag catcacaaa atcgacgctc aagtcagagg

241 tggcgaaacc cgacaggact ataaagatac caggcgtttc cccctggaag ctccctcgtg

301 cgctctcctg ttccgaccet gccgcttacc ggatacctgt ccgccttet cccttcggga

361 agcgtggcgc tttctcatag ctcacgctgt aggtatctca gttcggtgta ggtcgttcgc

421 tccaagctgg gctgtgtgca cgaacccccc gttcagcccg accgctgcgc cttatccggt

481 aactatcgtc ttgagtccaa cccggtaaga cacgacttat cgccactggc agcagccact

541 ggtaacagga ttagcagagc gaggtatgta ggcggtgcta cagagttctt gaagtggtgg

601 cctaactacg gctacactag aagaacagta tttggtatct gcgctctgct gaagccagtt

661 accttcggaa aaagagttgg tagctcttga tccggcaaac aaaccaccgc tggtagcggt

721 ggttttttg tttgcaagca gcagattacg cgcagaaaaa aaggatctca agaagatcct

781 ttgatcttt ctacggggtc tgacgctcag tggaacgaaa actcacgtta agggattttg

841 gtcatgagat tatcaaaag gatcttcacc tagatccttt taaattaaaa atgaagttt

901 aatcaatct aagtatata tgagtaaact tggtctgaca gttaccaatg cttaatcagt

961 gaggcaccta tctcagcgat ctgtctattt cgttcatcca tagttgcctg actccccgtc

1021 gtgtagataa ctacgatacg ggagggctta ccatctggcc ccagtgctgc aatgataccg

1081 cgagacccac gctcaccggc tccagattta tcagcaataa accagccagc cggaagggcc

1141 gagcgcagaa gtggtcctgc aactttatcc gcctccatcc agtctattaa ttgttgccgg

1201 gaagctagag taagtagttc gccagttaat agtttgcgca acgttgttgc cattgctaca

1261 ggcatcgtgg tgtcacgctc gtcgtttggt atggcttcat tcagctccgg ttcccaacga

1321 tcaaggcgag ttacatgatc ccccatgttg tgcaaaaag cggttagctc cttcggtcct

1381 ccgatcgttg tcagaagtaa gttggccgca gtgttatcac tcatggttat ggcagcactg

1441 cataattctc ttactgtcat gccatccgta agatgctttt ctgtgactgg tgagtactca

1501 accaagtcat tctgagaata gtgtatgcgg cgaccgagtt gctcttgcce ggcgtcaata

1561 cgggataata ccgcgccaca tagcagaact ttaaaagtgc tcatcattgg aaaacgttct

1621 tcggggcgaa aactctcaag gatcttaccg ctgttgagat ccagttcgat gtaacccact 
1681 cgtgcaccca actgatcttc agcatctttt actttcacca gcgtttctgg gtgagcaaaa

1741 acaggaaggc aaaatgccgc aaaaaaggga ataagggcga cacggaaatg ttgaatactc

1801 atactcttcc tttttcaata ttattgaagc atttatcagg gttattgtct catgagcgga

1861 tacatatttg aatgtattta gaaaataaa caaatagggg ttccgcgcac atttccccga

1921 aaagtgccac ctgacgtcta agaaaccatt attatcatga cattaaccta taaaatagg

1981 cgtatcacga ggcccttco tctcgcgcgt ttcggtgatg acggtgaaaa cctctgacac

2041 atgcagctcc cggagacggt cacagcttgt ctgtaagcgg atgccgggag cagacaagcc

2101 cgtcagggcg cgtcagcggg tgttggcggg tgtcggggct ggcttaacta tgcggcatca

2161 gagcagattg tactgagagt gcaccataaa attgtaaacg ttaatatttt gttaaaattc

2221 gcgttaaatt tttgttaaat cagctcattt tttaaccaat aggccgaaat cggcaaaatc

2281 ccttataaat caaaagaata gcccgagata gggttgagtg ttgttccagt ttggaacaag

2341 agtccactat taaagaacgt ggactccaac gtcaaagggc gaaaaccgt ctatcagggc

2401 gatggcccac tacgtgaacc atcacccaaa tcaagttttt tggggtcgag gtgccgtaaa

2461 gcactaaatc ggaaccctaa agggagcccc cgatttagag cttgacgggg aaagccggcg

2521 aacgtggcga gaaaggaagg gaagaaagcg aaaggagcgg gcgctagggc gctggcaagt

2581 gtagcggtca cgctgcgcgt aaccaccaca cccgccgcgc ttaatgcgcc gctacagggc

2641 gcgtactatg gttgctttga cgtatgcggt gtgaaatacc gcacagatgc gtaaggagaa

2701 aataccgcat caggcgccat tcgccattca ggctgcgcaa ctgttgggaa gggcgatcgg

2761 tgcgggcctc ttcgctatta cgccagctgg cgaaagggg atgtgctgca aggcgattaa

2821 gttgggtaac gccagggttt tcccagtcac gacgttgtaa aacgacggcc agtgccagct

2881 ggccggccta ggcgcgccaa gcttaaggtg cacggcccac gtggccacta gtgagcagcc

2941 agttgttttt ttctatattc ctgatgggca tacgaaggag agcccgtttg cgcttaatac

3001 tgccaaggcc acgactaatg cggtaagttt tttattattt taaaatgaaa caacttttta

3061 tctttaaatt aaataattta ttaaaaattt ttaaataatc tacaaaatc agtctttaaa

3121 cttaatgcag tcactatcta ctttattata actaaagtaa ctttacgtac tattatttac

3181 aaaaataaac tatcacaatt aactaagtag gaacaaaaac cgatcatctc gagcggccgc

3241 tcatcagaaa cagctctggc acatgtcgat gttgtgctcc cggcagatga ccttcttaca

3301 cttcttgcag ctggcgctgg cctttcttct gatcttgctg ggacagtagg tgcagtaggt

3361 cctcttcttc atcacgggct cctcggtgct gtcgtcgctg gtgccgggca cctctttggg

3421 caggatgttg ctgatgttgt ccctcaggta tctcttcagg gtgggggcct ccagcctctt

3481 cctcatgaag ctggaggtca ggcccatgta caggttccgc atgaactttt tccggctctg

3541 caccttctcg cccttgctgc tcacgttgtg gctgtagatg atgaagctgt tgatgcaggc

3601 gatgttgatc atgccgtaca gcagggccat gggccacctg ttggtctttc tgctgcaggt

3661 catcacgctg cacatctggt ccagggtgtc cacgccgccc ttggtctggt tgtagtacat

3721 caccatctgg ggcttgccgg tgctctcgtt gatgctggcg tcctcgtcgc agctggacag

3781 caggtacacc atcttggcgg gcttgggctt gtaggacacc agggtcaggg ggccgtcgaa

3841 gcagaacatg ctggtgccca cgggcctgga cctgctgttc ttcaggacct cggggatctc

3901 tctcttgttg cttctcacgg tgcccacgat ggtcagcttg tagggctcct gcagcaggtt

3961 cttggccagg gggatgctgg tgaaccagtt gtcgcaggtg atgtttctgc agctgccgtg

4021 gacgggcttg gacagctcct tcacgtagta ctcgcccagg ggcacgccgt tggtctgggt

4081 gcccctgccc aggtagggca tgccgttgat catgtacttg gtgccoctgt cgcacatcat

4141 caggatcttg atgccgtact tgctgggctt gttggggata tagaccctga aggggcacct

4201 gccctgaag cccagcagct gctcgtcgat ggtcaggtgg gcgccagggg tgtagttctg

4261 gatgcactgg tggatgaaca ggtcccagat ctttctcacg ggggtgaaca cgtcgttctc

4321 ccgcagggtg ggcctgatgc tcttgtcgtc catcctcagg catctgatca ggaagtcgaa

4381 tctgtccctg ctcatcacgc tcacgtacac catgctcagg gatctgtcga acaggtcgtc

4441 ggtgctcatg tggttgtcct tcctcacggc ggtcatcacc aggatgccga agaaggcgta

4501 gatctcgtcc tcgttggtgt ccctgaaggt ggcggaggtc atgctctcc gcctcttcag

4561 gctgatctcg gcgttggtcc acttcacgat ctcgctgatg atctcgtcgg tgaagaacag

4621 cttgaagcac agcagggggt cgtagatgtt cctgcacatc ctggtggggc ccctctggct

4681 tctcacgatg ttcagggcgg acaccctgct ccgcctggtg ggcttggagg tggaccagca

4741 gtgcttgttc ttgcccctga tggtcctctg gggcagggtc aggatcctgt tgctggccag

4801 ggagctgccg ggctgctcga tcacgttctg ctcgtccagg atctcggagc cgctgctggt

4861 aggctgcacc tcgtgcacct cgtcgatgaa ggcctcctcg gtgtcggact gcacgtcgtc

4921 ctcgctcacg tggtcgctca cctcgctgtc gctgtcctcg ccgaccagct cgtcgtcget

4981 ctgcagcagg gcgctcagga tgtgctcgtc gtccaggctg ctgcccatgg tggcggcgaa

5041 ttccagcaca ctggcggccg ttactagtgg atccgagctc ggtacctcta gatgcacttt

5101 tcgatttact ttgaattcac tagtaaataa ttcactcaac tttgttaaag tcgctttgaa

5161 aatttgcttt gagtcgcttg cttagctttg ttgctttcgc ttcgcttgat tcaaattcac

5221 tgacaacgcg ccgcgagacc gcgcttatat atgaaacggg gaattcgtct cgaacttcac

5281 cgaatgatct cgaagtttcc attgatttgc gtttgaattt tctcgaattt ttatttttaa

5341 aacggccttt tcggtattat tcatgctatt tccgtaaaga taaaggagaa tagtggaaac

5401 tggttgtgcc ctaatttatt tcaacttcca aataaaaac aatcattata tattaatgca

5461 tttaaaaaa aaacgttaca taacgaatga gttgttgact atttcatgtt tatttttaat

5521 ttggtaaagt tcggcaatat tatgaatgag tagtgaattg ttttgatttc atatacatat

5581 gccaaagttg gattgtcata gcaattttaa taaacaatt ttcctatttt ttttctttt

5641 aaacttatta ataggtcttt aagtaaaatg ttttagatta atcctttttt ttacttttat

5701 cgtttataat caattacttt tgcagaagat gcctaaataa tagtgattaa taataaataa

5761 agaacaattt tttattgaag gttgggatcc atatataggg cccgggttat aattacctca

5821 ggtcgacgtc ccatggccat tcgaattcgg ccggcctagg cgcgccgtaa tcatggtcat

5881 agctgtttcc tgtgtgaaat tgttatccgc tcacaltcc acacaacata cgagccggaa

5941 gcataaagtg taaagcctgg ggtgcctaat gagtgagcta actcacatta attgcgttgc

6001 gctcactgcc cgctttccag tcgggaaacc tgtcgtgcca gctgcattaa tgaatcggcc

6061 aacgcgcggg gagaggcggt ttgcgtattg ggcgc 

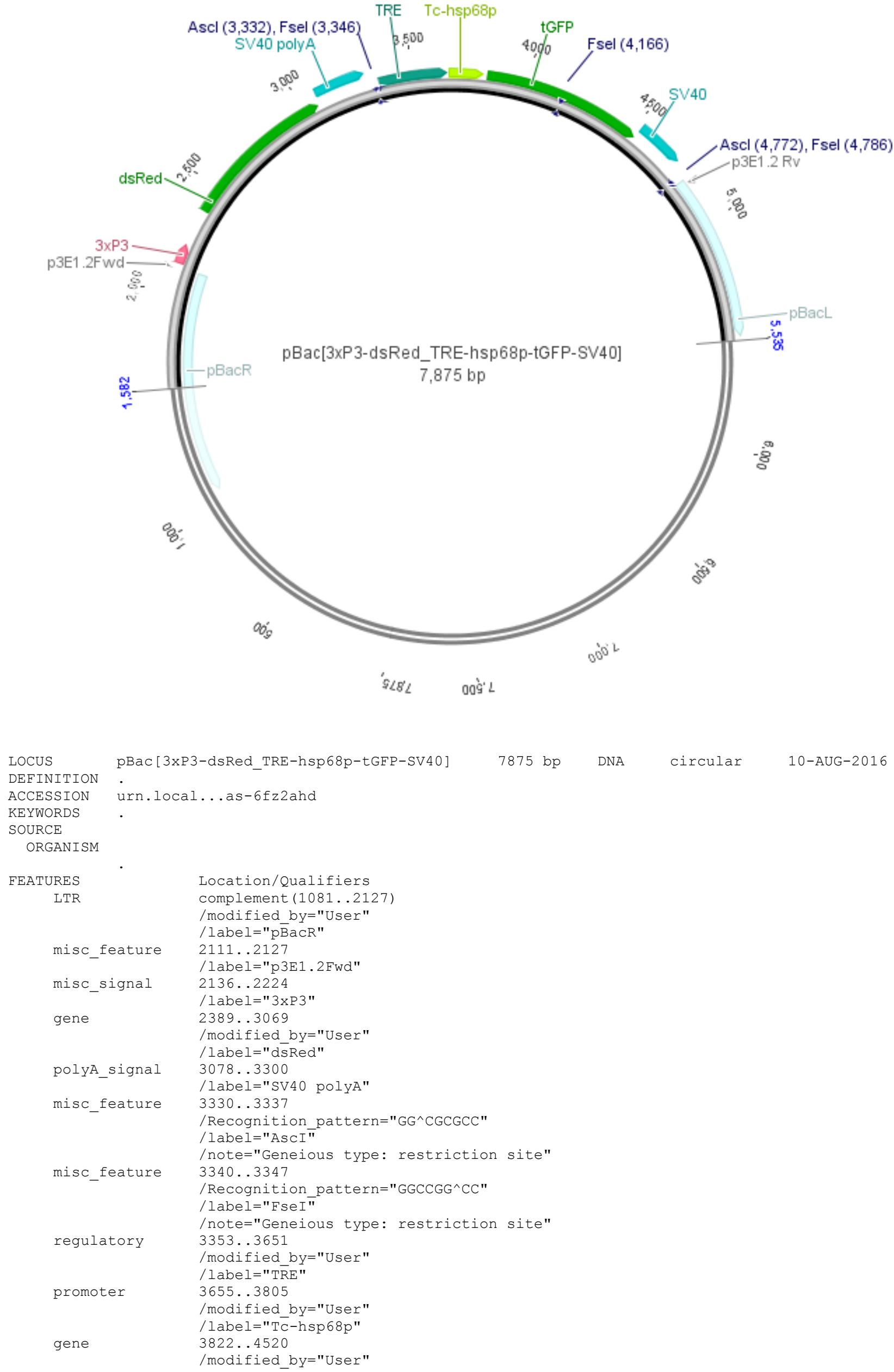
/ label="tGFP"

misc_feature $\quad 4160 \ldots 4167$

/Recognition_pattern="GGCCGG^CC"

/ label="FseI"

/note="Geneious type: restriction site"

polyA_signal 4520..4724

/modified by="User"

/ label="SV $40 "$

misc_feature $\quad 4770 \ldots 4777$

/Recognition_pattern="GG^CGCGCC"

/ label="AscI"

/note="Geneious type: restriction site"

misc_feature $\quad 4780 \ldots 4787$

/Recognition pattern="GGCCGG^CC"

/ label="FseI"

/note="Geneious type: restriction site"

LTR

4795.5517

/modified_by="User"

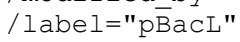

misc feature 4818..4838

/ label="p3E1.2 Rv"

ORIGIN

1 tcgcgcgttt cggtgatgac ggtgaaaacc tctgacacat gcagctcccg gagacggtca

61 cagcttgtct gtaagcggat gccgggagca gacaagcccg tcagggcgcg tcagcgggtg

121 ttggcgggtg tcggggctgg cttaactatg cggcatcaga gcagattgta ctgagagtgc

181 accatatgcg gtgtgaaata ccgcacagat gcgtaaggag aaaataccgc atcaggcgcc

241 attcgccatt caggctgcgc aactgttggg aagggcgatc ggtgcgggcc tcttcgctat

301 tacgccagct ggcgaaaggg ggatgtgctg caaggcgatt aagttgggta acgccagggt

361 tttcccagtc acgacgttgt aaaacgacgg ccagtgccaa gctttgttta aaatataaca

421 aaattgtgat cccacaaaat gaagtggggc aaatcaaat aattaactag tgtccgtaaa

481 cttgttggtc ttcaactttt tgaggaacac gttggacggc aaatcgtgac tataacacaa

541 gttgatttaa taattttagc caacacgtcg ggctgcgtgt tttttgcgct ctgtgtacac

601 gttgattaac tggtcgatta aataatttaa tttttggttc ttctttaaat ctgtgatgaa

661 attttttaaa ataactttaa attcttcatt ggtaaaaaat gccacgtttt gcaacttgtg

721 agggtctaat atgaggtcaa actcagtagg agttttatcc aaaaagaaa acatgattac

781 gtctgtacac gaacgcgtat taacgcagag tgcaaagtat aagagggtta aaaatatat

841 tttacgcacc atatacgcat cgggttgata tcgttaatat ggatcaattt gaacagttga

901 ttaacgtgtc tctgctcaag tctttgatca aaacgcaaat cgacgaaaat gtgtcggaca

961 atatcaagtc gatgagcgaa aaactaaaa ggctagaata cgacaatctc acagacagcg

1021 ttgagatata cggtattcac gacagcaggc tgaataataa aaaattaga aactattatt

1081 taaccctaga aagataatca tattgtgacg tacgttaaag ataatcatgc gtaaaattga

1141 cgcatgtgtt ttatcggtct gtatatcgag gtttatttat taatttgaat agatattaag

1201 ttttattata tttacactta catactaata ataattcaa caacaatt atttatgttt

1261 atttatttat taaaaaaaa caaaactca aaatttcttc tataaagtaa caaaactttt

1321 aaacattctc tcttttacaa aaataaactt attttgtact ttaaaacag tcatgttgta

1381 ttataaaata agtaattagc ttaacttata cataatagaa acaaattata cttattagtc

1441 agtcagaaac aactttggca catatcaata ttatgctctc gacaaataac ttttttgcat

1501 tttttgcacg atgcatttgc ctttcgcctt attttagagg ggcagtaagt acagtaagta

1561 cgttttttca ttactggctc ttcagtactg tcatctgatg taccaggcac ttcatttggc

1621 aaaatattag agatattatc gcgcaaatat ctcttcaaag taggagcttc taaacgctta

1681 cgcataaacg atgacgtcag gctcatgtaa aggtttctca taaatttttt gcgactttga

1741 accttttctc ccttgctact gacattatgg ctgtatataa taaagaatt tatgcaggca

1801 atgtttatca ttccgtacaa taatgccata ggccacctat tcgtcttcct actgcaggtc

1861 atcacagaac acatttggtc tagcgtgtcc actccgcctt tagtttgatt ataatacata

1921 accatttgcg gtttaccggt actttcgttg atagaagcat cctcatcaca agatgataat

1981 aagtatacca tcttagctgg cttcggttta tatgagacga gagtaagggg tccgtcaaa

2041 caaaacatcg atgttcccac tggcctggag cgactgtttt tcagtacttc cggtatctcg

2101 cgtttgtttg atcgcacggt tcccacatg cggggattat tcattagaga ctaattcaat

2161 tagagctaat tcaattagga tccaagctta tcgatttcga accctcgacc gccggagtat

2221 aaatagaggc gcttcgtcta cggagcgaca attcaattca aacaagcaaa gtgaacacgt

2281 cgctaagcga aagctaagca aataaacaag cgcagctgaa caagctaaac aatcggggta

2341 ccgctagagt cgacggtacc gcgggcccgg gatccaccgg tcgccaccat ggtgcgctcc

2401 tccaagaacg tcatcaagga gttcatgcgc ttcaaggtgc gcatggaggg caccgtgaac

2461 ggccacgagt tcgagatcga gggcgagggc gagggccgcc cctacgaggg ccacaacacc

2521 gtgaagctga aggtgaccaa gggcggcccc ctgccettcg cctgggacat cctgtccccc

2581 cagttccagt acggctccaa ggtgtacgtg aagcaccccg ccgacatccc cgactacaag

2641 aagctgtcct tccccgaggg cttcaagtgg gagcgcgtga tgaacttcga ggacggcggc

2701 gtggtgaccg tgacccagga ctcctccctg caggacggct gettcatcta caaggtgaag

2761 ttcatcggcg tgaacttccc ctccgacggc cccgtaatgc agaagaagac catgggctgg

2821 gaggcctcca ccgagcgcet gtacccccgc gacggcgtgc tgaagggcga gatccacaag

2881 gccetgaagc tgaaggacgg cggccactac ctggtggagt tcaagtccat ctacatggcc

2941 aagaagcccg tgcagctgcc cggctactac tacgtggact ccaagctgga catcacctcc

3001 cacaacgagg attacaccat cgtggagcag tacgagcgca ccgagggccg ccaccacctg

3061 ttcctgtagc ggccgcgact ctagatcata atcagccata ccacatttgt agaggtttta

3121 cttgctttaa aaacctccc acacttcccc ctgaacctga aacataaaat gaatgcaatt

3181 gttgttgtta acttgtttat tgcagcttat aatggttaca aataaagcaa tagcatcaca

3241 aatttcacaa ataaagcatt tttttcactg cattctagtt gtggtttgtc caaactcatc

3301 atgtatctta agcttatcga tacgcotacg gcgcgcctag gccggccgaa tttcgagttt

3361 accactccct atcagtgata gagaaaagtg aaagtcgagt ttaccactcc ctatcagtga

3421 tagagaaag tgaaagtcga gtttaccact ccctatcagt gatagagaaa agtgaaagtc 
3481 gagtttacca ctccctatca gtgatagaga aagtgaaag tcgagtttac cactccctat 3541 cagtgataga gaaagtgaa agtcgagttt accactccct atcagtgata gagaaagtg 3601 aaagtcgagt ttaccactcc ctatcagtga tagagaaag tgaaagtcga gctccgtttc 3661 atatataagc gcggtctcgc ggcgcgttgt cagtgaattt gaatcaagcg aagcgaaagc 3721 aacaaagcta agcaagcgac tcaaagcaaa ttttcaaagc gactttaaca aagttgagtg 3781 aattatttac tagtgaattc aaagtggtac cggtcgccac catggagagc gacgagagcg 3841 gcctgcccgc catggagatc gagtgccgca tcaccggcac cctgaacggc gtggagttcg 3901 agctggtggg cggcggagag ggcacccccg agcagggccg catgaccaac aagatgaaga 3961 gcaccaaagg cgccctgacc ttcagcccct acctgctgag ccacgtgatg ggctacggct 4021 tctaccactt cggcacctac cccagcggct acgagaaccc cttcctgcac gccatcaaca 4081 acggcggcta caccaacacc cgcatcgaga agtacgagga cggcggcgtg ctgcacgtga 4141 gcttcagcta ccgctacgag gccggccgcg tgatcggcga cttcaaggtg atgggcaccg 4201 gcttccccga ggacagcgtg atcttcaccg acaagatcat ccgcagcaac gccaccgtgg 4261 agcacctgca ccccatgggc gataacgatc tggatggcag cttcacccgc accttcagcc 4321 tgcgcgacgg cggctactac agctccgtgg tggacagcca catgcacttc aagagcgcca 4381 tccaccccag catcctgcag aacgggggcc ccatgttcgc cttccgccgc gtggaggagg 4441 atcacagcaa caccgagctg ggcatcgtgg agtaccagca cgccttcaag accccggatg 4501 cagatgccgg tgaagaataa agcggccgcg actctagatc ataatcagcc ataccacatt 4561 tgtagaggtt ttacttgctt taaaaacct cccacacctc cccctgaacc tgaacataa 4621 aatgaatgca attgttgttg ttaacttgtt tattgcagct tataatggtt acaataaag 4681 caatagcatc acaaatttca caaataaagc attttttca ctgcattcta gttgtggttt 4741 gtccaaactc atcaatgtat cttaagcttg gcgcgcctag gccggccgat ctcggatctg 4801 acaatgttca gtgcagagac tcggctacgc ctcgtggact ttgaagttga ccaacaatgt 4861 ttattcttac ctctaatagt cctctgtggc aaggtcaaga ttctgttaga agccaatgaa 4921 gaacctggtt gttcaataac attttgttcg tctaatattt cactaccgct tgacgttggc 4981 tgcactcat gtacctcatc tataaacgct tcttctgtat cgctctggac gtcatcttca 5041 cttacgtgat ctgatatttc actgtcagaa tcctcaccaa caagctcgtc atcgctttgc 5101 agaagagcag agaggatatg ctcatcgtct aagaactac ccattttatt atatattagt 5161 cacgatatct ataacaagaa aatatatata taataagtta tcacgtaagt agaacatgaa 5221 ataacalat aattatcgta tgagttaal cttaaaagtc acgtaaaaga taatcatgcg 5281 tcattttgac tcacgcggtc gttatagttc aaatcagtg acacttaccg cattgacaag 5341 cacgcctcac gggagctcca agcggcgact gagatgtcct aaatgcacag cgacggattc 5401 gcgctattta gaaagagaga gcaatatttc aagaatgcat gcgtcaattt tacgcagact 5461 atctttctag ggttaaaaa gatttgcgct ttactcgacc taaactttaa acacgtcata 5521 gaatcttcgt ttgacaaaa ccacattgtg gccaagctgt gtgacgcgac gcgcgctaaa 5581 gaatggcaaa ccaagtcgcg cgagcgtcga ctctagagga tccccgggta ccgagctcga 5641 attcgtaatc atggtcatag ctgtttcctg tgtgaaattg ttatccgctc acaatccac 5701 acaacatacg agccggaagc ataaagtgta aagcctgggg tgcctaatga gtgagctaac 5761 tcacattaat tgcgttgcgc tcactgcccg ctttccagtc gggaaacctg tcgtgccagc 5821 tgcattaatg aatcggccaa cgcgcgggga gaggcggttt gcgtattggg cgctcttccg 5881 cttcctcgct cactgactcg ctgcgctcgg tcgttcggct gcggcgagcg gtatcagctc 5941 actcaaaggc ggtaatacgg ttatccacag aatcagggga taacgcagga aagaacatgt 6001 gagcaaaagg ccagcaaaag gccaggaacc gtaaaaaggc cgcgttgctg gcgtttttcc 6061 ataggctccg cccccctgac gagcatcaca aaalcgacg ctcaagtcag aggtggcgaa 6121 acccgacagg actataaaga taccaggcgt ttccccctgg aagctccctc gtgcgctctc 6181 ctgttccgac cctgccgctt accggatacc tgtccgcctt tctcccttcg ggaagcgtgg 6241 cgctttctca atgctcacgc tgtaggtatc tcagttcggt gtaggtcgtt cgctccaagc 6301 tgggctgtgt gcacgaaccc cccgttcagc ccgaccgctg cgccttatcc ggtaactatc 6361 gtcttgagtc caacccggta agacacgact tatcgccact ggcagcagcc actggtaaca 6421 ggattagcag agcgaggtat gtaggcggtg ctacagagtt cttgaagtgg tggcctaact 6481 acggctacac tagaaggaca gtatttggta tctgcgctct gctgaagcca gttaccttcg 6541 gaaaagagt tggtagctct tgatccggca aacaaaccac cgctggtagc ggtggtttt 6601 ttgtttgcaa gcagcagatt acgcgcagaa aaaaggatc tcaagaagat cctttgatct 6661 tttctacggg gtctgacgct cagtggaacg aaactcacg ttaagggatt ttggtcatga 6721 gattatcaaa aaggatcttc acctagatcc ttttaaatta aaaatgaagt tttaatcaa 6781 tctaagtat atatgagtaa acttggtctg acagttacca atgcttaatc agtgaggcac 6841 ctatctcagc gatctgtcta tttcgttcat ccatagttgc ctgactcccc gtcgtgtaga 6901 taactacgat acgggagggc ttaccatctg gccccagtgc tgcaatgata ccgcgagacc 6961 cacgctcacc ggctccagat ttatcagcaa taaaccagcc agccggaagg gccgagcgca 7021 gaagtggtcc tgcaactta tccgcctcca tccagtctat taattgttgc cgggaagcta 7081 gagtaagtag ttcgccagtt aatagtttgc gcaacgttgt tgccattgct acaggcatcg 7141 tggtgtcacg ctcgtcgttt ggtatggctt cattcagctc cggttcccaa cgatcaaggc 7201 gagttacatg atcccccatg ttgtgcaaaa aagcggttag ctccttcggt cctccgatcg 7261 ttgtcagaag taagttggcc gcagtgttat cactcatggt tatggcagca ctgcataatt 7321 ctcttactgt catgccatcc gtaagatgct tttctgtgac tggtgagtac tcaaccaagt 7381 cattctgaga atagtgtatg cggcgaccga gttgctcttg cccggcgtca atacgggata 7441 ataccgcgcc acatagcaga actttaaaag tgctcatcat tggaaaacgt tcttcggggc 7501 gaaactctc aaggatctta ccgctgttga gatccagttc gatgtaaccc actcgtgcac 7561 ccaactgatc ttcagcatct tttactttca ccagcgtttc tgggtgagca aaacaggaa 7621 ggcaaaatgc cgcaaaaaag ggaataaggg cgacacggaa atgttgaata ctcatactct 7681 tccttttca atattattga agcatttatc agggttattg tctcatgagc ggatacatat 7741 ttgaatgtat ttagaaaal aaacaaatag gggttccgcg cacatttccc cgaaaagtgc 7801 cacctgacgt ctaagaaacc attattatca tgacattaac ctataaaat aggcgtatca 7861 cgaggccett tcgtc 

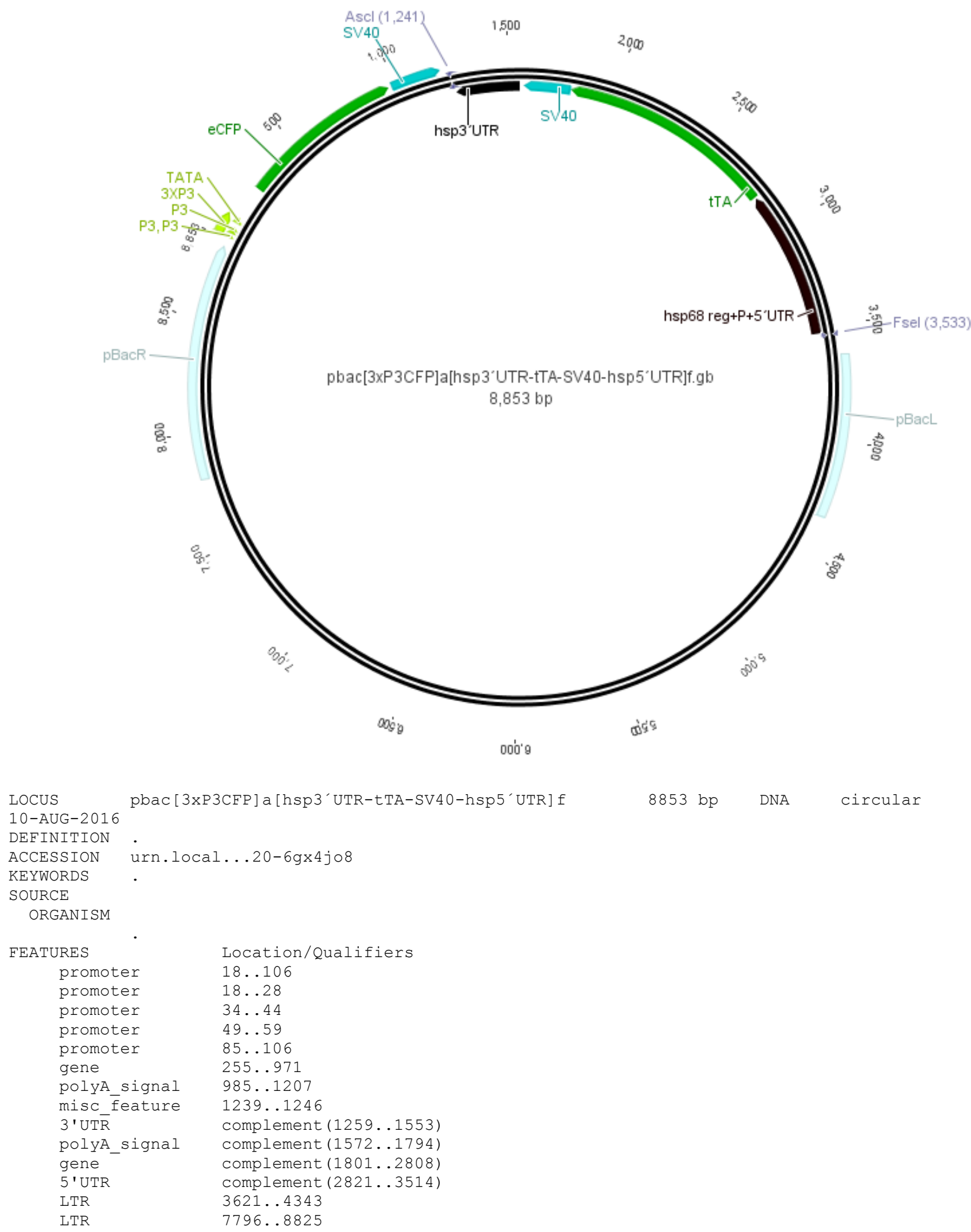

1 gagctcgccc ggggatctaa ttcaattaga gactaattca attagagcta attcaattag 61 gatccaagct tatcgatttc gaaccctcga ccgccggagt ataaatagag gcgcttcgtc 121 tacggagcga caattcaatt caaacaagca aagtgaacac gtcgctaagc gaaagctaag 181 caaataaaca agcgcagctg aacaagctaa acaatcgggg taccgctaga gtcgacgatc 241 caccggtcgc caccatggtg agcaagggcg aggagctgtt caccggggtg gtgcccatcc 301 tggtcgagct ggacggcgac gtaaacggcc acaagttcag cgtgtccggc gagggcgagg 361 gcgatgccac ctacggcaag ctgaccctga agttcatctg caccaccggc aagctgcccg 421 tgccctggcc caccetcgtg accaccetga cctggggcgt gcagtgcttc agccgctacc 481 ccgaccacat gaagcagcac gacttcttca agtccgccat gcccgaaggc tacgtccagg 541 agcgcaccat cttcttcaag gacgacggca actacaagac ccgcgccgag gtgaagttcg 601 agggcgacac cctggtgaac cgcatcgagc tgaagggcat cgacttcaag gaggacggca 661 acatcctggg gcacaagctg gagtacaact acatcagcca caacgtctat atcaccgccg 721 acaagcagaa gaacggcatc aaggccaact tcaagatccg ccacaacatc gaggacggca 781 gcgtgcagct cgccgaccac taccagcaga acacccceat cggcgacggc cccgtgctgc 
841 tgcccgacaa ccactacctg agcacccagt ccgccctgag caaagacccc aacgagaagc

901 gcgatcacat ggtcctgctg gagttcgtga ccgccgccgg gatcactctc ggcatggacg

961 agctgtacaa gtaaagcggc cgcgactcta gatcataatc agccatacca catttgtaga

1021 ggttttactt getttaaaa acctcccaca cctccccctg aacctgaaac ataaatgaa

1081 tgcaattgtt gttgttaact tgtttattgc agcttataat ggttacaat aaagcaatag

1141 catcacaal ttcacaaata aagcatttt ttcactgcat tctagttgtg gtttgtccaa

1201 actcatcaat gtatcttaaa gcttatcgat acgcgtacgg cgcgccgtgg ccactagtga

1261 gcagccagtt gttttttct atattcctga tgggcatacg aaggagagcc cgtttgcgct

1321 taatactgcc aaggccacga ctaatgcggt aagtttttta ttattttaaa atgaaacaac

1381 ttttatctt taaattaaat aatttattaa aaatttttaa ataatctaca aaatcagtc

1441 tttaactta atgcagtcac tatctacttt attataacta aagtaacttt acgtactatt

1501 atttacaaaa ataaactatc acaattaact aagtaggaac aaaaaccgat catctcgagc

1561 catgggccgc gactctagat cataatcagc cataccacat ttgtagaggt tttacttgct

1621 ttaaaaacc tcccacacct ccccctgaac ctgaaacata aaatgaatgc aattgttgtt

1681 gttaacttgt ttattgcagc ttataatggt tacaaataaa gcaatagcat cacaatttc

1741 acaaataaag cattttttc actgcattct agttgtggtt tgtccaaact catcccatgg

1801 ctacccaccg tactcgtcaa ttccaagggc atcggtaaac atctgctcaa actcgaagtc

1861 ggccatatcc agagcgccgt agggggcgga gtcgtggggg gtaaatcccg gacccgggga

1921 atccccgtcc cccaacatgt ccagatcgaa atcgtctagc gcgtcggcat gcgccatcgc

1981 cacgtcctcg ccgtctaagt ggagctcgtc ccccaggctg acatcggtcg ggggggccgt

2041 cgacagtctg cgcgtgtgtc ccgcggggag aaaggacagg cgcggagccg ccagccccgc

2101 ctcttcgggg gcgtcgtcgt ccgggagatc gagcaggccc tcgatggtag acccgtaatt

2161 gttttcota cgcgcgcggc tgtacgcgga cccactttca catttaagtt gttttctaa

2221 tccgcatatg atcaattcaa ggccgaataa gaaggctggc tctgcacctt ggtgatcaaa

2281 taattcgata gcttgtcgta ataatggcgg catactatca gtagtaggtg tttcccttc

2341 ttcttagcg acttgatgct cttgatcttc caatacgcaa cctaaagtaa aatgccccac

2401 agcgctgagt gcatataatg cattctctag tgaaaacct tgttggcata aaaaggctaa

2461 ttgattttcg agagtttcat actgttttc tgtaggccgt gtacctaal gtacttttgc

2521 tccatcgcga tgacttagta aagcacatct aaactttta gcgttattac gtaaaaatc

2581 ttgccagct tcccctcta aagggcaaaa gtgagtatgg tgcctatcta acatctcaat

2641 ggctaaggcg tcgagcaaag cccgcttatt ttttacatgc caatacaatg taggctgctc

2701 tacacctagc ttctgggcga gtttacgggt tgttaaacct tcgattccga cctcattaag

2761 cagctctaat gcgctgttaa tcacttact tttatctaat ctactcatt tgctagctag

2821 atgcacttt cgatttactt tgaattcact agtaaataat tcactcaact ttgttaagt

2881 cgcttgaaa atttgctttg agtcgcttgc ttagctttgt tgctttcgct tcgcttgatt

2941 caattcact gacaacgcgc cgcgagaccg cgcttatata tgaaacgggg aattcgtctc

3001 gaacttcacc gaatgatctc gaagtttcca ttgatttgcg tttgaattt ctcgaattt

3061 tattttaaa acggccttt cggtattatt catgctattt ccgtaaagat aaaggagaat

3121 agtggaaact ggttgtgccc taatttatt caacttccaa aataaaaca atcattatat

3181 attaatgcat ttaaaaaaa aacgttacat aacgaatgag ttgttgacta tttcatgttt

3241 atttttaatt tggtaaagtt cggcaatatt atgaatgagt agtgaattgt tttgatttca

3301 tatacatatg ccaaagttgg attgtcatag caattttaat aaaacaatt tcctatttt

3361 tttctttta aacttattaa taggtcttta agtaaatgt tttagattaa tccttttt

3421 tacttttatc gtttataatc aattactttt gcagaagatg cctaaataat agtgattaat

3481 aataaataaa gaacaatttt ttattgaagg ttgggatcag cttaagggcc ggccgaattc

3541 gaatggccat gggacgtcga cctgaggtaa ttataacccg ggccctatat atggatccaa

3601 ttgcaatgat catcatgaca gatctgacaa tgttcagtgc agagactcgg ctacgcctcg

3661 tggactttga agttgaccaa caatgtttat tcttacctct aatagtcctc tgtggcaagg

3721 tcaagattct gttagaagcc aatgaagaac ctggttgttc aataacattt tgttcgtcta

3781 atatttcact accgcttgac gttggctgca cttcatgtac ctcatctata aacgcttctt

3841 ctgtatcgct ctggacgtca tcttcactta cgtgatctga tatttcactg tcagaatcct

3901 caccaacaag ctcgtcatcg ctttgcagaa gagcagagag gatatgctca tcgtctaaag

3961 aactacccat tttattatat attagtcacg atatctataa caagaaata tatatataat

4021 aagttatcac gtaagtagaa catgaataa caatataatt atcgtatgag ttaatctta

4081 aagtcacgt aaagataat catgcgtcat tttgactcac gcggtcgtta tagttcaaaa

4141 tcagtgacac ttaccgcatt gacaagcacg cctcacggga gctccaagcg gcgactgaga

4201 tgtcctaaat gcacagcgac ggattcgcgc tatttagaaa gagagagcaa tatttcaaga

4261 atgcatgcgt caattttacg cagactatct ttctagggtt aaaaaagatt tgcgctttac

4321 tcgacctaaa ctttaaacac gtcatagaat cttcgtttga caaaaaccac attgtggcca

4381 agctgtgtga cgcgacgcgc gctaaagaat ggcaaaccaa gtcgcgcgag cgtcgactct

4441 agaggatccc cgggtaccga gctcgaattc gtaatcatgg tcatagctgt ttcctgtgtg

4501 aattgttat ccgctcacaa ttccacacaa catacgagcc ggaagcataa agtgtaaagc

4561 ctggggtgcc taatgagtga gctaactcac attaattgcg ttgcgctcac tgcccgcttt

4621 ccagtcggga aacctgtcgt gccagctgca ttaatgaatc ggccaacgcg cggggagagg

4681 cggtttgcgt attgggcgct cttccgcttc ctcgctcact gactcgctgc gctcggtcgt

4741 tcggctgcgg cgagcggtat cagctcactc aaaggcggta atacggttat ccacagatc

4801 aggggataac gcaggaaaga acatgtgagc aaaaggccag caaaaggcca ggaaccgtaa

4861 aaaggccgcg ttgctggcgt ttttccatag getccgcccc cctgacgagc atcacaaaa

4921 tcgacgctca agtcagaggt ggcgaaaccc gacaggacta taaagatacc aggcgtttcc

4981 ccctggaagc tccctcgtgc getctcctgt tccgaccctg ccgcttaccg gatacctgtc

5041 cgccttctc cettcgggaa gcgtggcgct ttctcaatgc tcacgctgta ggtatctcag

5101 ttcggtgtag gtcgttcgct ccaagctggg ctgtgtgcac gaaccccccg ttcagcccga

5161 ccgctgcgcc ttatccggta actatcgtct tgagtccaac ccggtaagac acgacttatc

5221 gccactggca gcagccactg gtaacaggat tagcagagcg aggtatgtag gcggtgctac

5281 agagttcttg aagtggtggc ctaactacgg ctacactaga aggacagtat ttggtatctg

5341 cgctctgctg aagccagtta ccttcggaaa aagagttggt agctcttgat ccggcaaca

5401 aaccaccgct ggtagcggtg gttttttgt ttgcaagcag cagattacgc gcagaaaaa

5461 aggatctcaa gaagatcctt tgatctttc tacggggtct gacgctcagt graacgaaaa

5521 ctcacgttaa gggattttgg tcatgagatt atcaaaagg atcttcacct agatccttt

5581 aattaaaa tgaagttt a atcaatcta aagtatatat gagtaaact ggtctgacag 
5641 ttaccaatgc ttaatcagtg aggcacctat ctcagcgatc tgtctatttc gttcatccat 5701 agttgcctga ctccccgtcg tgtagataac tacgatacgg gagggcttac catctggccc 5761 cagtgctgca atgataccge gagacccacg ctcaccggct ccagatttat cagcaataaa 5821 ccagccagcc ggaagggccg agcgcagaag tggtcctgca actttatccg cctccatcca 5881 gtctattaat tgttgccggg aagctagagt aagtagttcg ccagttaata gtttgcgcaa 5941 cgttgttgcc attgctacag gcatcgtggt gtcacgctcg tcgtttggta tggcttcatt 6001 cagctccggt tcccaacgat caaggcgagt tacatgatcc cccatgttgt gcaaaaagc 6061 ggttagctcc ttcggtcctc cgatcgttgt cagaagtaag ttggccgcag tgttatcact 6121 catggttatg gcagcactgc ataattctct tactgtcatg ccatccgtaa gatgctttc 6181 tgtgactggt gagtactcaa ccaagtcatt ctgagaatag tgtatgcggc gaccgagttg 6241 ctcttgcccg gcgtcaatac gggataatac cgcgccacat agcagaactt taaagtgct 6301 catcattgga aaacgttctt cggggcgaaa actctcaagg atcttaccgc tgttgagatc 6361 cagttcgatg taacccactc gtgcacccaa ctgatcttca gcatcttta ctttcaccag 6421 cgtttctggg tgagcaaaaa caggaaggca aaatgccgca aaaaagggaa taagggcgac 6481 acggaaatgt tgaatactca tactcttcct ttttcaatat tattgaagca tttatcaggg 6541 ttattgtctc atgagcggat acatatttga atgtatttag aaaataaac aaataggggt 6601 tccgcgcaca tttccccgaa aagtgccacc tgacgtctaa gaaaccatta ttatcatgac 6661 attaacctat aaaataggc gtatcacgag gccettcot ctcgcgcgtt tcggtgatga 6721 cggtgaaaac ctctgacaca tgcagctccc ggagacggtc acagcttgtc tgtaagcgga 6781 tgccgggagc agacaagccc gtcagggcgc gtcagcgggt gttggcgggt gtcggggctg 6841 gcttaactat gcggcatcag agcagattgt actgagagtg caccatatgc ggtgtgaaat 6901 accgcacaga tgcgtaagga gaaataccg catcaggcgc cattcgccat tcaggctgcg 6961 caactgttgg gaagggcgat cggtgcgggc ctcttcgcta ttacgccagc tggcgaaagg 7021 gggatgtgct gcaaggcgat taagttgggt aacgccaggg ttttcccagt cacgacgttg 7081 taaacgacg gccagtgcca agctttgttt aaaatataac aaaattgtga tcccacaaaa 7141 tgaagtgggg caaaatcaaa taattaatag tgtccgtaaa cttgttggtc ttcaactttt 7201 tgaggaacac gttggacggc aaatccgtga ctataacaca agttgattta ataattttag 7261 ccaacacgtc gggctgcgtg ttttttgccg acgcgtctgt gtacacgttg attaactggt 7321 cgattaaact gttgaaataa tttaattttt ggttcttctt taaatctgtg atgaaattt 7381 ttaaaataac tttaaattct tcattggtaa aaaatgccac gttttgcaac ttgtgagggt 7441 ctaatatgag gtcaaactca gtaggagttt tatccaaaaa agaaaacatg attacgtctg 7501 tacacgaacg cgtattaacg cagagtgcaa agtataagag ggttaaaaa tatattttac 7561 gcaccatata cgcatcgggt tgatatcgtt aatatggatc aatttgaaca gttgattaac 7621 gtgtctctgc tcaagtcttt gatcaaacg caaatcgacg aaaatgtgtc ggacalatc 7681 aagtcgatga gcgaaaact aaaaggcta gaatacgaca atctcacaga cagcgttgag 7741 atatacggta ttcacgacag caggctgaat aataaaaaa ttagaaacta ttatttaacc 7801 ctagaaagat aatcatattg tgacgtacgt taaagataat catgcgtaaa attgacgcat 7861 gtgttttatc ggtctgtata tcgaggttta tttattaatt tgaatagata ttaagtttta 7921 ttatatttac acttacatac taataataaa ttcaacaaac aatttattta tgtttattta 7981 tttattaaaa aaaacaaaa actcaaatt tcttctataa agtaacaaa cttttaaca 8041 ttctctcttt tacaaaala aacttatttt gtactttaaa aacagtcatg ttgtattata 8101 aaataagtaa ttagcttaac ttatacataa tagaacaaa ttatacttat tagtcagtca 8161 gaaacaactt tggcacatat caatattatg ctctcgacaa ataacttttt tgcattttt 8221 gcacgatgca tttgccttc gccttattt agaggggcag taagtacagt aagtacgttt 8281 tttcattact ggctctcag tactgtcatc tgatgtacca ggcacttcat ttggcaaat 8341 attagagata ttatcgcgca atatctctt caaagtagga gcttctaaac gcttacgcat 8401 aaacgatgac gtcaggctca tgtaaaggtt tctcataaat tttttgcgac tttggacctt 8461 ttctcccttg ctactgacat tatggctgta tataataaa gaatttatgc aggcaatgtt 8521 tatcattccg tacaataatg ccataggcca cctattcgtc ttcctactgc aggtcatcac 8581 agaacacatt tggtctagcg tgtccactcc gcctttagtt tgattataat acataaccat 8641 ttgcggttta ccggtacttt cgttgataga agcatcctca tcacaagatg ataataagta 8701 taccatctta gctggcttcg gtttatatga gacgagagta aggggtccgt caaacaaaa 8761 catcgatgtt cccactggcc tggagcgact gttttcagt acttccggta tctcgcgttt 


\subsection{Danksagung}

An erster Stelle möchte ich mich bei meiner Familie für den bedingungslosen Rückhalt bedanken, welcher es mir ermöglicht hat so weit zu kommen.

Spezieller Dank gebührt meiner Frau Carolin, die mich in den vergangen Jahren großartig unterstützt hat.

Ich danke Prof. Dr. Ernst A. Wimmer, nicht nur für die Möglichkeit diese Arbeit in seiner Abteilung durchzuführen, sondern auch für die herzliche Betreuung, für die kompetente Anleitung meiner Arbeit, für die vielen Gespräche die mein wissenschaftliches Denken und Verständnis geprägt haben und dafür, dass er menschlich stets ein Vorbild war.

Ich danke Prof. Dr. André Fiala für seine guten Ratschläge und die kritische Begutachtung meiner Arbeit.

Besonder Dank gilt Prof. Dr. Joachim Schachtner, der mich schon als Student für den Geruchssinn begeistert hat und stets ein wichtiger Ansprechpartner war und ist.

Ich danke Prof. Dr. Sigrid Hoyer-Fender und Prof. Dr. Gregor Bucher für die vielen konstruktiven und angenehmen Gespräche.

Ein besonderer Dank gilt allen TAs und Sekretärinnen der Abteilung, ohne die diese Arbeit nicht möglich gewesen wäre!

Ebenfalls möchte ich mich bei Dr. Sergio Angeli, Prof. Dr. Stefan Schütz, Karthi Balakrishnan, Dr. Jörg Kahnt und allen anderen Kooperationspartnern für die gute und erfolgreiche Zusammenarbeit bedanken.

Besonderer Dank gilt auch allen Kollegen, die mich auf dem Weg begleitet haben, für die schöne Zeit im Labor, die vielen Diskussionen und die entspannte Atmosphäre. Danke an Dawid, Georg, Kolja, Alice, Janna, Natascha, Ingrid, Montse, Peter, Bernhard und den ganzen Rest der Bande, es war mir eine Freude!

Ein ganz großer Dank geht an Dr. Martin Kollmann, der mich seit vielen Jahren als Freund und Kooperationspartner durch dieses Projekt begleitet hat!

Vielen, herzlichen Dank! 


\subsection{Publications}

Dreyer, D., Vitt, H., Dippel, S., Goetz, B., El Jundi, B., Kollmann, M., Huetteroth, W., and Schachtner, J. (2010). 3D Standard Brain of the Red Flour Beetle Tribolium Castaneum: A Tool to Study Metamorphic Development and Adult Plasticity. Front. Syst. Neurosci. 4, 3.

Paczkowski, S., Paczkowska, M., Dippel, S., Schulze, N., Schütz, S., Sauerwald, T., Weiß, A., Bauer, M., Gottschald, J., and Kohl, C.-D. (2013). The olfaction of a fire beetle leads to new concepts for early fire warning systems. Sens. Actuators B Chem. 183, 273-282.

Paczkowski, S., Paczkowska, M., Dippel, S., Flematti, G., and Schütz, S. (2014). Volatile Combustion Products of Wood Attract Acanthocnemus nigricans (Coleoptera: Acanthocnemidae). J. Insect Behav. 27, 228-238.

Dippel, S., Oberhofer, G., Kahnt, J., Gerischer, L., Opitz, L., Schachtner, J., Stanke, M., Schütz, S., Wimmer, E.A., and Angeli, S. (2014). Tissue-specific transcriptomics, chromosomal localization, and phylogeny of chemosensory and odorant binding proteins from the red flour beetle Tribolium castaneum reveal subgroup specificities for olfaction or more general functions. BMC Genomics 15, 1141.

Eckermann, K.N., Dippel, S., KaramiNejadRanjbar, M., Ahmed, H.M., Curril, I.M., and Wimmer, E.A. (2014). Perspective on the combined use of an independent transgenic sexing and a multifactorial reproductive sterility system to avoid resistance development against transgenic Sterile Insect Technique approaches. BMC Genet. 15 Suppl 2, S17.

Dippel, S., Kollmann, M., Oberhofer, G., Montino, A., Knoll, C., Kral, M., Rexer, K., Frank, S., Schachtner, J., and Wimmer E.A. (2016). Morphological and Transcriptomic Analysis of a Beetle Chemosensory System Reveals a Gnathal Olfactory Center. BMC Biology. 2016;14:90. 